\title{
Movimiento Browniano en Cadenas de Osciladores Anarmónicos Unidimensionales
}

\author{
TESIS
}

\author{
Que presenta
M. en C. Mauricio Romero Bastida Mat. 94351361 \\ para obtener el grado de \\ Doctor en Ciencias
}

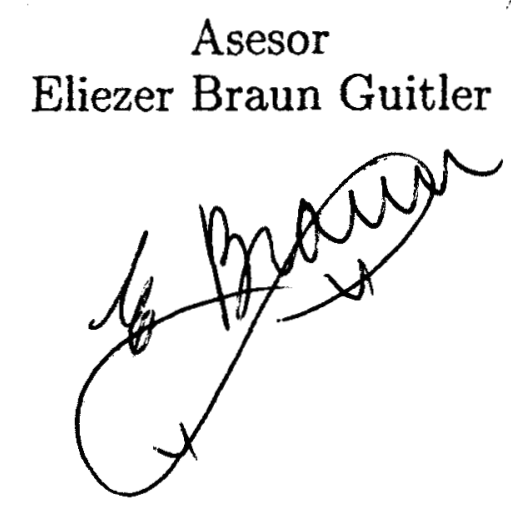

México, D.F. Agosto de 2002

DEPARTAMENTO DE FÍSICA

DIVISIÓN DE CIENCIAS BÁSICAS E INGENIERÍA

UNIVERSIDAD AUTÓNOMA METROPOLITANA

UNIDAD IZTAPALAPA 
A la Virgen del Rayo, por haber oido mis plegarias. 


\section{Agradecimientos}

A mis padres Guillermo y Lidia, que siempre me impulsaron y apoyaron para salir adelante.

A mi hermana Claudia, un apoyo constante aunque silencioso a lo largo de todos estos años.

Al Dr. Eliezer Braun, por la oportunidad de trabajar bajo su dirección y por proponerme un excelente proyecto de investigación.

A los sinodales, Dr. Leopoldo García-Colín, Dr. Eduardo Piña, Dr. Salvador Godoy y Dr. Jose Luís Mateos, por sus valiosos comentarios.

Al Doctor Enrique Gonzalez Tovar por haberme facilitado las primeras versiones de los programas de Dinámica Molecular con los que se realizaron la mayor parte de los cálculos de este trabajo y a Francisco Parada Rabell por su invaluable ayuda para preparar la presentación en PowerPoint.

A mi amigo José Antonio Gonzalez Avante, el cual me dió, a su manera, una nueva perspectiva de la vida sin la cual no hubiera podido llegar hasta donde he llegado.

Al CONACyT por el apoyo económico brindado para la realización de mis estudios de posgrado y al SNI por la ayuda posteriormente recibida.

A la UAM-I, por todo el apoyo recibido. 


\section{RESUMEN}

El problema de la relación entre el caos microscópico y el comportamiento macroscópico de un sistema de muchos grados de libertad se explora numéricamente por medio del estudio de las propiedades estadísticas asociadas a la posición y el momento de una impureza pesada acoplada a una cadena de osciladores con interacciones anarmónicas a primeros vecinos del tipo Fermi-Pasta-Ulam. Para este modelo particular hemos encontrado que el comportamiento del tiempo de relajación asociado a la función de autocorrelación del momento es diferente dependiendo del régimen dinámico (ya sea regular o caótico) de la cadena. Los principales resultados del presente trabajo han sido reportados en : M. Romero-Bastida y E. Braun, "Microscopic chaos from Brownian motion in a onedimensional anharmonic oscillator chain," Phys. Rev. E 65, 036228 (2002) [ver también http://arXiv.org/abs/nlin/0201059]. 


\section{ABSTRACT}

The problem of relating microscopic chaos to macroscopic behavior in a many-degrees-of-freedom system is numerically investigated by analyzing statistical properties associated to the position and momentum of a heavy impurity embedded in a chain of nearestneighbor anharmonic Fermi-Pasta-Ulam oscillators. For this particular model we have found that the behavior of the relaxation time of the momentum autocorrelation function of the impurity is different depending on the dynamical regime (either regular of chaotic) of the lattice. The main results of this work have been reported in: $M$. Romero-Bastida and E. Braun, "Microscopic chaos from Brownian motion in a one-dimensional anharmonic oscillator chain," Phys. Rev. E 65, 036228 (2002) http://arXiv.org/abs/nlin/0201059 


\section{Índice General}

1 Introducción 1

2 Movimiento Browniano 5

2.1 Introducción . . . . . . . . . . . . . . . . 5

2.2 Antecedentes Históricos . . . . . . . . . . . . . . . 6

2.3 Fenomenología . . . . . . . . . . . . . . . . 12

2.3.1 Ecuación de Langevin . . . . . . . . . . . . 14

2.3.2 Ecuación de Fokker-Planck . . . . . . . . . 18

2.4 Modelos Microscópicos . . . . . . . . . . . . . . . . . . 19

2.4.1 Modelo de Fluido: Primeros Trabajos . . . . . . . . . . . 19

2.4.2 Modelo de Fluido: Desarrollos Posteriores . . . . . . . . . 25

2.5 Conclusiones . . . . . . . . . . . . . . . . . . 26

3 Modelos de Osciladores Acoplados 29

3.1 Introducción . . . . . . . . . . . . . . . . . . . . . . . . 29

3.2 Interacciones a Primeros Vecinos . . . . . . . . . . . . . 30

3.2.1 Autocorrelación de $P_{0}(t) \ldots \ldots . \ldots 33$

3.2.2 Autocorrelación de $f(t) \ldots \ldots . \ldots 37$

3.2.3 Ecuación de Langevin . . . . . . . . . . . . 39

3.3 Interacciones de Largo Alcance . . . . . . . . . . . . . . . . . . 40

3.4 Conclusiones . . . . . . . . . . . . . . . . . . 46

4 El Modelo Fermi-Pasta-Ulam $\quad \mathbf{4 9}$

4.1 Introducción . . . . . . . . . . . . . . . . . . 49

4.2 El Problema de la Equipartición . . . . . . . . . . . . . 50

4.2 .1 El Trabajo Original . . . . . . . . . . . . . 51

4.2 .2 Relevancia Física del Modelo FPU . . . . . . . . . . 53 
4.2.3 Resultados Posteriores .............. . . 55

4.3 Dinámica de Sistemas Hamiltonianos . . . . . . . . . . . . . 58

4.3.1 Teoremas Generales . . . . . . . . . . . . . 59

4.3.2 Resultados Analíticos Particulares . . . . . . . . . . 63

4.3.3 Resultados Numéricos ... . . . . . . . . . . 64

4.4 Conclusiones....................... 66

5 El Modelo FPU Modificado $\quad 69$

5.1 Introducción . . . . . . . . . . . . . . . 69

5.2 Planteamiento del Modelo . . . . . . . . . . . 70

5.3 Implementación y Validación del Modelo . . . . . . . . . . . 71

5.3 .1 Conservación de la Energía . . . . . . . . . . 74

5.3 .2 Parámetro de Control ............. 76

5.4 Equilibración del Sistema . . . . . . . . . . . 79

5.4.1 Primeros Resultados ............... 81

5.4 .2 Resultados en el Límite Termodinárnico . . . . . . . . . 87

5.5 Resultados Adicionales . . . . . . . . . . . . . . 94

5.6 Conclusiones. . . . . . . . . . . . . . . . . . 99

6 Movimiento Browniano en una Cadena FPU 101

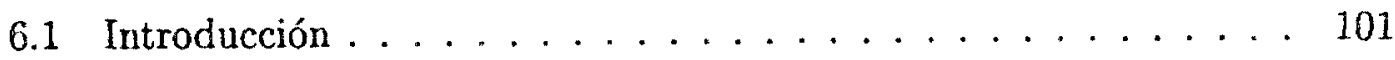

6.2 Validación: El Caso Armónico . . . . . . . . . . . . . . 102

6.2.1 Desplazamiento Cuadrático Medio . . . . . . . . . . 102

6.2.2 Autocorrelación del Momento . . . . . . . . . . . 105

6.2.3 Fuerza sobre el Oscilador Pesado ............ 109

6.3 Resultados en el Régimen Anarmónico . . . . . . . . . . . 111

6.3.1 Coeficiente de Difusión . . . . . . . . . . . 112

6.3 .2 Tiempo de Relajación $\tau \ldots \ldots . \ldots . \ldots 115$

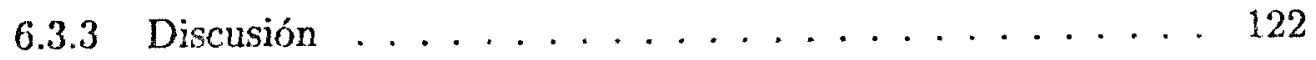

7 Conclusiones y Perspectivas $\quad 127$

A Obtención del Conjunto Completo $\left\{U_{i}(k)\right\}$

B Correspondencia entre Modelos de Corto y Largo Alcance 139 
C El Modelo de Mazur y Braun (1964) 143

C.1 Dinámica del oscilador pesado en un "baño térmico" . . . . . . 143

C.2 Límite del Acoplamiento Débil . . . . . . . . . . . . . 147

$\begin{array}{ll}\text { D Algoritmo de Integración } & 155\end{array}$

$\begin{array}{ll}\text { Bibliografia } & 161\end{array}$ 


\section{Capítulo 1}

\section{Introducción}

La teoría cinética de los gases fue desarrollada a finales del siglo XIX, siendo la primera sistematización rigurosa, aunque restringida a un cierto tipo de sistemas muy específicos, de la teoría atómica de la materia. Sin embargo, esta teoría no era del todo aceptada debido a la falta de evidencia experimental directa del movimiento a nivel molecular. Einstein arguyó que la la trayectoria errática de una pequeña partícula suspendida en un fluido (fenómeno que se conoce como movimiento Browniano) es el resultado de las colisiones aleatorias con las moléculas que constituyen el fluido. Según la explicación propuesta por Einstein el comportamiento de la partícula suspendida debería de mostrar, en promedio, algún tipo de regularidad. Este comportamiento efectivamente se manifiesta en una cantidad conocida como desplazamiento cuadrático medio, la cual se incrementa linealmente con el tiempo (ver Ec. (2.10) del capítulo siguiente). A continuación, Einstein mostró cómo el factor de proporcionalidad está relacionado con el número de Avogadro; esto es, con el número de moléculas que, de acuerdo a la teoría atómica, deberían de colisionar con la partícula suspendida. Aunque estas predicciones fueron comprobadas experimentalmente por Perrin algunos años después, confirmando así la hipótesis molecular, la teoría antes mencionada se basa en una suposición crucial. En su desarrollo, Einstein simplemente supuso que las colisiones sucesivas con las moléculas del fluido deberían de ser estadísticamente independientes. La posición de la partícula suspendida es esencialmente la suma de variables aleatorias independientes, por lo que, de acuerdo a la teoría matemática de los procesos estocásticos, el resultado es un proceso difusivo cuya característica más fundamental es la dependencia lineal del desplazamiento cuadrático medio con respecto al tiempo, como ya lo habíamos mencionado. Pero un análisis real- 
mente microscópico debería de estudiar a la partícula suspendida junto con el fluido como un sistema dinámico de muchos grados de libertad, gobernado por las ecuaciones de Newton. Entonces esta dinámica microscópica determinista de bería, de alguna manera, poder explicar la independencia estadística que Einstein supuso a nivel fenomenológico.

Ahora bien, de los estudios actuales sobre la dinámica de los sistemas de muchos grados de libertad se sabe que la gran mayoria de estos son caóticos'; esto es, su dinámica tiene propiedades muy diferentes a las de sus contrapartes integrables. Sería natural suponer entonces que, como el fluido en el que está sumergida la partícula macroscópica del moviniento Browniano es un sistcma esencialmente caótico, el efecto de la dinámica microscópica debería de alguna manera de ser cuantificable de manera experimental. Gaspard et al. [1] realizaron el primer experimento directo para tratar de encontrar evidencia en este sentido. Para una esfera de plástico sumergida en agua en equilibrio termodinámico estos autores encontraron, midiendo las posiciones sucesivas de la esfera y calculando a partir de éstas la entropía de Kolmogorov-Sinai $h_{k s}$ (una cantidad que, si es positiva, indica en principio la presencia de una dinámica caótica en un sistema dado), la primera evidencia, en la forma de una $h_{k s}$ positiva y acotada, de un comportamiento caótico a nivel microscópico que se manifiesta a nivel macroscópico.

Sin embargo, en un estudio posterior de E. G. D. Cohen et al. [2] por medio de simulaciones numéricas de un sistema no-caótico (el modelo de viento-árbol de Ehrenfest) pudieron reproducir numéricamente el comportamiento de $h_{K S}$ reportado por Gaspard et al., lo que hace que los resultados experimentales de [1] no sean tan concluyentes como en principio pudiera parecer. Resultados posteriores $[3,4]$, obtenidos por medio de la comparación del comportamiento del desplazamiento cuadrático medio, principalmente, de sistemas no-caóticos y caóticos (entre estos últimos el modelo de Lorent $z^{2}$ ), han mostrado que los efectos de la dinámica microscópica de estos sistemas sobre las propiedades difusivas no son detectables, por lo que existe una duda razonable de que, por medio de la metodología experi-

\footnotetext{
${ }^{i}$ En este punto de la exposición sólo es necesario saber que las trayectorias en el espacio fase de un sistema de este tipo tienen un caracter marcadamente no-periódico. Para una descripción mas rigurosa, ver el capítulo 4

${ }^{2}$ Los modelos utilizados en $[2,3,4]$ consisten en una partícula puntual moviéndose con velocidad unitaria en dos dimensiones que colisiona con dispersores que no se translapan. Los modelos tipo Ehrenfest (regulares) consisten en dispersores cuadrados distribuidos en posiciones ya sea periódicas o aleatorias en el plano, mientras que los modelos tipo Lorentz (caóticos) consisten en dispersores circulares.
} 
mental presentada en [1], sea posible encontrar evidencia de caos dinámico a nivel microscópico.

El hecho de que en modelos microscópicos no-caóticos se presente la fenomenología del movimiento Browniano no es de ninguna manera novedoso. Los modelos conocidos genéricamente como de osciladores armónicos acoplados, consistentes en una partícula pesada sumergida en un retículo de partículas livianas, comparten con los modelos utilizados por Cohen et al. la propiedad de tener una dinámica regular (esto es, no-caótica), lo que permite un estudio analítico del problema. A pesar de que son modelos muy simplificados, son lo suficientemente poderosos como para ser un punto de partida adecuado en la tarea de esclarecer muchas de las hipótesis necesarias para poder recuperar al movimiento Browniano macroscópico.

La principal limitante de este tipo de modelos es su excesiva simplificación en cuanto al tipo de interacciones que existen entre las partículas del sistema, ya que ésta es de caracter armónico. Sin embargo, su estructura puede generalizarse por medio de la inclusión de potenciales anarmónicos, lo que permite, en principio, responder la pregunta: ¿cuáles son las modificaciones introducidas en el comportamiento macroscópico de la partícula pesada debido al efecto de una dinámica microscópica de caracter caótico? A pesar de ser un programa de investigación inmediato, hasta el presente no se ha intentado llevarlo a cabo, y este trabajo es un primer paso en esta dirección.

Ahora bien, una ventaja de nuestro enfoque es que, como nuestro punto de partida es un modelo no-caótico que permite obtener analíticamente la fenomenología propia del movimiento Browniano, la generalización al caso caótico nos permite asegurar que las desviaciones del comportamiento armónico son el resultado de la dinámica caótica que hemos impuesto, por construcción, a nuestro sistema. En principio sería entonces posible estudiar, desde un punto de vista distinto pero complementario al de Cohen et al., la posibilidad de que en la dinámica macroscópica de la partícula pesada se manifiesten, de alguna manera, los efectos de la dinámica microscópica subyacente.

Los diferentes conceptos tratados en este capítulo son explicados a lo largo de la tesis como sigue.

En el capítulo 2 se hará una revisión, de ninguna manera exhaustiva, del movimiento Browniano desde el punto de vista fenomenológico, así como una discusión de los primeros intentos de una formulación microscópica (Hamiltoniana) para el caso de una partícula pesada sumergida en un fluido molecular. Estudi- 
con el enfoque de Boltzmann desde una perspectiva histórica, ver Ref. [7]).

Podemos ver que, en general, para pasar de una descripción totalmente microscópica (hamiltoniana) a una totadnente macroscópica (termodinámica) tiene que haber una reducción de los grados de libertad involucrados, obteniéndose así una descripcion en la cual un conjunto de variables reducidas obedecen ecuaciones fenomenológicas bien conocidas. En general es muy difícil conseguir esta reducción, pero existe un fenómeno físico para el cual ésta se consigue de una manera bastante sencilla. Este fenómeno es el movimiento Browniano, en el cual se estudia el movimiento de una partícula macroscópica suspendida en un fluido. Para este problema las únicas variables macroscópica relevantes son aquellas asociadas a la partícula suspendida, mientras que las variables asociadas a las partículas que componen al fluido entran en la descripción indirectamente a través de sus efectos estocásticos sobre la partícula suspendida. Lo anterior fue mostrado por Einstein a principios de siglo, obteniendo una de las primeras y más concluyentes demostraciones del alcance de los métodos de la mecánica estadística.

La descripción de este esfuerzo y su continuación por Paul Langevin algunos años después será el primer objetivo de este capítulo. Posteriormente haremos una breve revisión de las herramientas teóricas que han sido desarrolladas para estudiar al movimiento Browniano de manera fenomenológica. Por último haremos un recuento de los modelos que han sido diseñados para tratar de obtener la fenomenología del movimiento Browniano a partir de modelos microscópicos, concluyendo con una ponderación de los alicances y limitaciones de este tipo de metodología.

\subsection{Antecedentes Históricos}

En el año de 1828 el botánico inglés Robert Brown reportó las observaciones, hechas el año anterior, sobre el movimiento errático de una suspensión de granos de polen en agua. Una bella descripción de este fenómeno fue dada posteriormente por Jean Perrin en los siguientes términos: “... sería difícil examinar durante mucho tiempo una preparación de particulas muy finas en un líquido sin observar un movimiento perfectamente irregular. Se mueven, se detienen, empiezan de nuevo, suben, bajan, suben otra vez sin que se vea que tiendan a la inmovilidad [8]." Cabe mencionar que este tipo de movimiento ya había sido observado muchas veces con anterioridad (Leeuwenhoek, Stephen Grant, Needham, Buffon, Spallanzani, entre otros). El gran mérito de Brown fue el de afirmar por primera vez que este 
fenómeno tenía causas físicas, no biológicas (la referencia al trabajo original de Brown, así como a los trabajos anteriores, puede consultarse en el capítulo 15 del libro de Brush [9]).

Durante el resto del siglo XIX se sugirieron todo tipo de posibles explicaciones: gradientes de temperatura, evaporación, corrientes de aire, flujo de calor, capilaridad, ósmosis, ninguna de las cuales resultó satisfactoria (ver Ref. [9] y Nota I en [11]). Uno de los pocos resultados concluyentes obtenidos en este período es que este movimiento no depende del tipo de partícula suspendida ni del tipo de fluido utilizado ${ }^{1}$. Gradualmente fue ganando terreno la hipótesis del origen molecular del movimiento Browniano (ya conocido por este nombre en la literatura de la época), la cual postula que éste se debe al gran número de colisiones que la partícula suspendida experimenta con las partículas microscópicas que componen al fluido. El resultado neto del gran número de colisiones es una fuerza que cambia brusca e intermitentemente, por lo que el movimiento que realiza la partícula Browniana se percibe macroscópicamente como errático. Es significativo de este período la ausencia total de publicaciones sobre el movimiento Browniano por los creadores de la teoría cinética tales como Clausius, Maxwell y Boltzmann. Sin embargo, podría sospecharse que éste último ya sabía en 1896 la causa de este movimiento errático cuando escribió "... partículas muy pequeñas en un gas ejecutan movimientos que resultan del hecho de que la presión en la superficie de las partículas puede fluctuar [10]". Los primeros intentos de aplicar la hipótesis de los impactos moleculares al movimiento Browniano resultaron infructuosos hasta que, en 1905, Einstein formuló una teoría capaz de orientar a los físicos experimentales sobre qué cantidad debía ser medida para así poder comprobar la hipótesis molecular [11].

¿Cuál fue el método seguido por Einstein? La idea general consiste en derivar una descripción probabilística válida para todo un conjunto de partículas, en lugar de seguir en el tiempo la complicada trayectoria de una sola de ellas. La primera suposición necesaria es que el movimiento de cada partícula es independiente del de las demás. La segunda suposición consiste en postular que la descripción deseada está definida por una escala de tiempo $\tau_{e}$ de tal modo que dos puntos diferentes de la trayectoria de una misma partícula, después de ese intervalo, pueden considerarse como eventos independientes. Además, no se hace ningún intento por caracterizar la dinámica en una escala de tiempo más pequeña que

\footnotetext{
${ }^{1}$ Este resultado experimental tiene consecuencias importantes que serán exploradas en el capítulo siguiente dentro del contexto de los modelos de osciladores acoplados.
} 
$\tau_{\epsilon}$. Una tercera suposición, de naturaleza probabilistica, es que existe una función de densidad de probabilidad (FDP) $f(\Delta)$ para una distancia $\Delta$ recorrida por la partícula Browniana durante el intervalo $\tau_{e} . f(\Delta)$ está definida de tal forma que $f(\Delta) d \Delta$ es la probabilidad de tener un cambio de posición dentro del intervalo $(\Delta, \Delta+d \Delta)$. Como $f(\Delta)$ es una FDP, debe de cumplir con

$$
\int_{-\infty}^{+\infty} f(\Delta) d \Delta=1
$$

Las únicas suposiciones necesarias para el desarrollo posterior son que $f(\Delta)$ sólo difiera de cero para valores muy pequeños de $\Delta$ y que satisfaga la condición de simetría

$$
f(-\Delta)=f(\Delta)
$$

Consideremos a continuación un ensamble de $N$ partículas Brownianas, el cual está caracterizado por una densidad numérica $n(x, t)$ tal que $n(x, t) d x$ representa al número de partículas en el intervalo $(x, x+d x)$ al tiempo $t$. De la hipótesis de que las trayectorias separadas un intervalo $\tau_{e}$ son independientes se sigue que el número de partículas en $x$ al tiempo $t+\tau_{p}$ está dado por el número de partículas en $x-\Delta$ al tiempo $t$ multiplicado por la probabilidad $f(\Delta)$ de que la partícula pase de $x-\Delta$ a $x$ e integrado para todos los posibles valores de $\Delta$ :

$$
n\left(x, t+\tau_{e}\right)=\int_{-\infty}^{+\infty} n(x-\Delta, t) f(\Delta) d \Delta .
$$

Esta es la ecuación de evolución para al densidad numérica $n(x, t)$. Ahora, como $\tau_{e}$ es muy pequeño, se tiene que

$$
n\left(x, t+\tau_{0}\right) \approx n(x, t)+\tau_{e} \frac{\partial n(x, t)}{\partial t} .
$$

También podemos desarrollar $n(x-\Delta, t)$ en potencias de $\Delta$ como

$$
n(x-\Delta, t) \approx n(x, t)-\Delta \frac{\partial n(x, t)}{\partial x}+\frac{\Delta^{2} \partial^{2} n(x, t)}{2 !}+\ldots
$$

Sustituyendo estos últimos desarrollos en la Ec. (2.3) podemos escribir

$$
n+\frac{\partial n}{\partial t} \tau_{e}=n \int_{-\infty}^{+\infty} f(\Delta) d \Delta-\frac{\partial n}{\partial x} \int_{-\infty}^{+\infty} \Delta f(\Delta) d \Delta+\frac{\partial^{2} n}{\partial x^{2}} \int_{-\infty}^{+\infty} \frac{\Delta^{2}}{2} f(\Delta) d \Delta-\ldots
$$

En el lado derecho de (2.6) los sumandos impares en $\Delta$ se anulan debido a la condición de simetría (2.2). A continuación tomarnos en consideración sólo térninos de 
$O\left(\Delta^{2}\right)$. La integral del primer sumando se evalúa usando (2.1), mientras que del tercero definimos al coeficiente de difusión $D$ en términos del segundo momento. de la FDP $f(\Delta)$ como

$$
D=\frac{1}{2 \tau_{e}} \int_{-\infty}^{+\infty} \Delta^{2} f(\Delta) d \Delta=\frac{\left\langle\Delta^{2}\right\rangle}{2 \tau_{e}}
$$

de donde también definimos al desplazamiento cuadrático medio como $\left\langle\Delta^{2}\right\rangle \equiv$ $\left\langle x^{2}(t)\right\rangle$, siendo $\langle\cdots\rangle$ la representación del promedio sobre todos los posibles valores de $\Delta$. Vemos entonces que de (2.6) se obiene la ecuación de difusión

$$
\frac{\partial n}{\partial t}=D \frac{\partial^{2} n}{\partial x^{2}}
$$

Si la condición inicial es que todas las partículas estén localizadas en $x=0$, $n(x, t=0)=N \delta(x)$, la solución de esta ecuación es

$$
n(x, t)=\frac{N}{(4 \pi D t)^{1 / 2}} \exp \left(-x^{2} / 4 D t\right)
$$

Puesto que $n(x, t)$ tiene la forma de lo que matemáticamente se conoce como una distribución Gaussiana, se sigue que la posición promedio de la partícula Browniana es nula y que el desplazarniento cuadrático medio se incrementa linealmente con el tiempo, esto es:

$$
\left\langle\Delta^{2}\right\rangle=2 D t, \quad t \sim \tau_{e} .
$$

Tanto la distribución de desplazamientos (2.9) como la relación (2.10) fueron comprobadas experimentalmente por M. Seddig, T. Svedberg, V. Henri, J. Perrin y Chaudesaiges (ver Ref. [9]), validando así el esquema general de Einstein.

La siguiente contribución al estudio del movimiento Browniano fue reportada por M. von Smoluchowski [12] al año siguiente de la publicación del trabajo de Einstein, aunque sus resultados fueron obtenidos muchos años antes de la publicación del trabajo de éste último [9]. La importancia de este trabajo es que utilizó el concepto de trayectoria libre media para calcular $\left\langle\Delta^{2}\right\rangle$. Con esto logró tomar en cuenta el efecto de las colisiones microscópicas sobre la trayectoria de la partícula Browniana de una forma más detallada que en el tratamiento de Einstein, convirtiéndose así en la primera aplicación de la teoría cinética al estudio del movimiento Browniano. Siguiendo esta metodología Smoluchowski encontró una expresión para el desplazamiento cuadrático medio de la forma $\left\langle\Delta^{2}\right\rangle=\left(3^{3 / 2} / 4\right) D t$. Esta ligera discrepancia en el valor del coeficiente de difusión no debería sorprendernos del todo si recordamós que la teoría cinética está concebida para el estudio 
de gases diluidos; además, el propio Smoluchowski adoptó la relación (2.10) en trabajos posteriores [9].

Aunque el enfoque de Einstein ha sido muy exitoso, uno tiene que admitir que es muy fenomenologico y que no puede dar, de una manera intuitivamente simple al menos, una expresión explícita que permita el cálculo de la constante de difusión en términos de cantidades macruscópicas. Langevin [13] propuso una metodología diferente y que, en sus propias palabras, es "infinitamente más simple" que la de Einstein. Su propuesta consistió, esencialmente, en estudiar la trayectoria de una sola partícula Browniana y escribir la correspondiente ecuación de Newton, fuerza=masa $\times$ aceleración. Para describir adecuadamente la fenomenología ya conocida, Langevin propuso que la fuerza macroscópica total actuando sobre la partícula Browniana se puede descomponer en la suma de dos contribuciones: una fuerza sistemática, que cuantifica el efecto de la fricción viscosa y que depende de la velocidad según la ley de Stokes, y una fuerza $F(t)$ ("fuerza complementaria" en su terminología) que da origen a la trayectoria errática de la partícula Browniana. Entonces la ecuación de movimiento para la partícula Browniana se puede escribir. como

$$
M \frac{d^{2} x}{d t^{2}}=-\gamma \frac{d x}{d t}+F(t)
$$

donde

$$
y=6 \pi \eta a,
$$

$\eta$ es la viscosidad del fluido y a es el radio de la partícula (supuesta esférica). Como veremos más adelante, la fuerza de fricción $-\gamma d x / d t$ y la fuerza fluctuante $F(t)$ no son independientes una de la otra. Esto es una consecuencia del hecho de que las colisiones moleculares que dan origen a estas fuerzas no pueden ser separadas en un tipo de colisión que dé origen sólo a un efecto de fricción y en otro tipo que dé origen sólo a un efecto fluctuante ${ }^{2}$. A continuación, Langevin supuso que la fuerza fluctuante $F(t)$ y la posición de la partícula Browniana están descorrelacionadas temporalmente:

$$
\langle F(t) x(t)\rangle=\langle x(t)\rangle\langle F(t)\rangle=0,
$$

donde $\langle\cdots\rangle$ denota un promedio tomado sobre todas las posibles realizaciones de la fuerza $F(t)$. Multiplicando la Ec. (2.11) por $x$, tomando el promedio anteriormente

\footnotetext{
${ }^{2}$ Para modelos simplificados [14] que tomen en cuenta de una manera sencilla los efectos de las colisiones moleculares individuales sobre la velocidad de la partícula Browniana, se obtienen resultados que reproducen exactamente las predicciones de la ecuación de Langevin, validando así la hipótesis de la separación de fijerzas en (2.11) para el caso particular de estos modelos.
} 
definido y utilizando la condición (2.13) se obtiene

$$
\frac{M}{2} \frac{d^{2}\left\langle x^{2}\right\rangle}{d t^{2}}-M\left\langle v^{2}\right\rangle=-3 \pi a \eta \frac{d\left\langle x^{2}\right\rangle}{d t}
$$

Langevin supuso a continuación que la partícula está en equilibrio termodinámico con el fluido circundante. Esto equivale a decir que el promedio de la energía cinética de la partícula Browniana es proporcional a la temperatura del fluido. Formalizando esta observación tenemos lo que se conoce como el principio de equipartición: $M\left\langle v^{2}\right\rangle=k_{B} T\left(k_{B}\right.$ es la constante de Boltzmann y $T$ es la temperatura del fluido). Si además utilizamos el cambio de variable $\xi=d\left\langle x^{2}\right\rangle / d t$, la ecuación (2.14) se puede escribir como

$$
\frac{M}{2} \frac{d \xi}{d t}+3 \pi a \eta \xi=k_{B} T
$$

cuya solución es

$$
\xi=\frac{k_{B} T}{3 \pi \eta a}+C \exp \left(-\frac{6 \pi \eta a}{M} t\right) .
$$

Después de un tiempo transitorio $\tau_{l} \equiv M / 6 \pi \eta a \simeq 10^{-8} \mathrm{~s}$, válido para partículas en las que se observa el movimiento Browniano, se entra a un régimen en el cual $\xi \simeq$ cte. Esto implica que la partícula no se ha movido en promedio $(\langle x\rangle=0) \mathrm{y}$ que el valor asintótico del desplazarniento cuadrático medio está dado por

$$
\left\langle x^{2}(t)\right\rangle=\frac{k_{B} T}{3 \pi \eta a} t, \quad t \gg \tau_{l}
$$

Este resultado no es otro que la relación de difusión de Einstein, pero ahora tenemos un valor explícito para el coeficiente de difusión:

$$
D=\frac{k_{B} T}{6 \pi \eta a} .
$$

Como hemos tenido ocasión de ver, el método de Langevin ofrece una forma más clara de cuantificar el efecto de las colisiones moleculares sobre la partícula Browniana a través de la fuerza fluctuante $F(t)$ junto con sus propiedades estadísticas. El método de Einstein no es tan explícito, pues el efecto de $F(t)$ es introducido a través de la FDP $f(\Delta)$. Sin embargo, ambas descripciones comparten un mismo formalismo matemático en términos de lo que actualmente se conocen como procesos estocásticos, los cuales son muy comunes en muchas áreas distintas de la física. De hecho, el formalismo de Einstein fue desarrollado independientemente por L. Bachelier para estudiar la especulación en el mercado de valores de París alrededor de 1900 [9]. 


\subsection{Fenomenología}

En la terminología moderna podemos decir que, tanto la posición en el análisis de Einstein como la velocidad en el de Langevin son procesos Markoffianos. Un proceso de este tipo está descrito por cualquier función del tiempo $\Theta(t)$ cuyo valor al tiempo $t+d t$ pueda determinarse probabilísticamente a partir de su valor en $t$, pero de tal modo que esta estimación no pueda ser mejorada con el conocimiento de valores de $\Theta(t)$ previos a $t$. Esta condición implica una forma muy específica de relacionar a $\Theta(t+d t) \operatorname{con} \Theta(t)$, la cual es [15]

$$
\Theta(t+d t)=\Theta(t)+A(\Theta(t), t) d t+D^{1 / 2}(\Theta(t), t) N(t)(d t)^{1 / 2}
$$

En esta ecuación $A(\Theta(t), t)$ y $D(\Theta(t), t)$ son funciones diferenciables en sus argumentos, conocidas como función de arrastre y función de difusión del proceso, respectivamente. $N(t)$ es una variable aleatoria normal con media 0 y varianza 1 , por lo que adoptamos la notación $N(t) \equiv N(0,1)$; como para cada instante se toma un valor distinto de la variable aleatoria, la dependencia temporal es implícita. Además, $N(t)$ está descorrelacionada temporalmente de $N\left(t^{\prime}\right)$ si $t \neq t^{\prime}$. La demostración de que efectivamente podemos describir la evolución temporal de un proceso Markoffiano por medio de (2.19) está detallada en la Ref. [15] y consiste en subdividir el intervalo $[t, t+d t)$ en $n \rightarrow \infty$ subintervalos iguales, imponiendo la condición de que la suma de los incrementos de $\Theta$ en esos subintervalos sea igual al incremento total $\Xi(\Theta(t), t ; d t) \equiv \Theta(t+d t)-\Theta(t)$ sobre el intervalo completo. Debido al teorema del límite central resulta que el incremento total es una variable aleatoria distribuida normalmente con media $A(\Theta(t), t) d t$ y varianza $D(\Theta(t), t) d t$; esto es, $\Xi(\Theta(t), t ; d t)=\mathbf{N}(A(\Theta(t), t) d t, D(\Theta(t), t) d t)$. Finalmente, utilizando la transformación funcional $\mathrm{N}\left(\alpha, \beta^{2}\right)=\alpha+\beta \mathrm{N}(0,1)$ junto con $\alpha \equiv A(\Theta(t), t) d t$ y $\beta \equiv D(\Theta(t), t) d t$ se llega al resultado $(2.19)^{3}$.

Un proceso Markoffiano cuyas funciones de arrastre y memoria tengan la forma

$$
A(\Theta(t), t)=-\frac{1}{\tau_{f}} \Theta(t) \quad y \quad D(\Theta(t), t)=c
$$

donde $\tau_{f}$ y $c$ sean dos constantes positivas se le conoce como un proceso de

\footnotetext{
${ }^{3}$ Operacionalmente puede considerarse que (2.19) define un algoritmo de integración para una ecuación diferencial estocástica en el que $d t$ juega el papel de un incremento temporal discreto $\Delta t$. Lo anterior ha sido mostrado explicitamente en un contexto totalmente diferente en [16]
} 
Ornstein-Uhlenbeck. Para el caso particular en que

$$
\tau_{f}=\frac{D M}{k_{B} T} \quad \text { y } \quad c=\frac{2}{D}\left(\frac{k_{B} T}{M}\right)^{2},
$$

la ecuación (2.13) corresponde a la ecuación de Langevin (2.11) escrita en términos de la velocidad de la partícula Browniana, $v(t)=d x / d t \equiv \Theta(t)$. La correspondiente coordenada de posición se se puede calcular a partir de

$$
x(t)-x(0)=\int_{0}^{t} v\left(t^{\prime}\right) d t^{\prime} .
$$

Por otra parte, si ahora se toman

$$
A(\Theta(t), t)=0 \quad \mathrm{y} \quad D(\Theta(t), t)=c^{*},
$$

de tal modo que $c^{*}>0$, se obtiene lo que se conoce como un proceso de Wiener sin arrastre con una constante de difusión $c^{*}$. En particular, si la variable aleatoria es la posición de la partícula Browniana, $\Theta(t) \equiv x(t)$, la ecuación de evolución que se obtiene a partir de (2.19) tiene la forma

$$
x(t+d t)=x(t)+\left(c^{*} d t\right)^{1 / 2} N(t)
$$

Ahora bien, resulta en general que la posición de la partícula Browniana calculada a partir de la integral (2.22) no coincide con el correspondiente resultado obtenido a partir de la ecuación de evolución (2.24) (la primera es una aproximación muy burda a la segunda). Sin embargo, si tomamos $\tau_{f} \rightarrow 0$ y $c \rightarrow \infty$ de tal modo que $\tau_{f} c^{1 / 2}=(2 D)^{1 / 2}=$ cte, entonces la posición de la partícula Browniana en el esquema de Langevin (calculada a partir de (2.22)) corresponde a un proceso Markoffiano que es estadísticamente indistinguible de la posición de esa misma partícula Browniana calculada según el esquema de Einstein si en (2.24) se hace la identificación $c^{*}=\tau_{f}^{2} c$ [17]. Esto último implica que la hipótesis de Einstein sobre la existencia de la escala de tiempo $\tau_{e}$ debe de entenderse en el sentido de que los cambios en la posición de la partícula Browniana ocurren en una escala de tiempo que es grande en comparación con aquelia en la que ocurren cambios significativos en la velocidad de la misma; esto es, $\tau_{e} \gg \tau_{l}$. Es en este sentido que la representación más general de la posición de una partícula Browniana está dada por la integral temporal de un proceso de Ornstein-Uhlenbeck, más que por un proceso de Wiener. 
En vista de lo anterior, primero veremos qué tanta información general puede deducirse a partir de la ecuación original de Langevin, pasando posteriormente a reseñar muy brevemente el fomalismo de la ecuación de Fokker-Planck, más cercano en su metodologia al enfoque de Einstein.

\subsubsection{Ecuación de Langevin}

En términos de la velocidad de la partícula Browniana, la ecuación de Langevin unidimensional se escribe como

$$
M \frac{d v}{d t}=-\gamma v+F(t)
$$

Debido a la presencia de $F(t)$, la anterior es una ecuación diferencial estocástica que no puede ser resuelta a menos que se hagan algunas suposiciones sobre la naturaleza estadística de $F(t)$. Puesto que ésta representa el efecto aleatorio de las colisiones a nivel molecular, suponemos que $F(t)$ es una variable estocástica distribuida Gaussianamente con un primer y un segundo momentos dados por

$$
\langle F(t)\rangle=0 \quad y \quad\left\langle F(t) F\left(t^{\prime}\right)\right\rangle=2 B \delta\left(t-t^{\prime}\right),
$$

donde $B$ es una constante cuyo significado se explicará más adelante $y\langle\cdots\rangle$ tiene el mismo significado que en la sección anterior. La presencia de la función delta de Dirac en el segundo momento es una consecuencia de la observación fenomenologica de que la escala de tiempo característica de las fluctuaciones (cambios de valor) de $F(t)$ es muy pequeña relativa a la escala de tiempo $r_{\text {c }}$ característica de la descripción de Langevin. Por lo tanto

$$
\langle F(t) v(0)\rangle=0 \quad t>0,
$$

la cual nos dice simplemente que, durante la misma escala de tiempo $\tau_{l}$, el cambio en $v$ es despreciable, mientras que $F$ ya ha experimentado numerosas fluctuaciones.

Bajo las suposiciones anteriores podemos ahora obtener varias predicciones interesantes a partir de (2.25). La solución formal de esta ecuación es

$$
v(t)=v(0) \exp (-\gamma t / M)+\frac{1}{M} \int_{0}^{t} \exp \left(-\gamma\left(t-t^{\prime}\right) / M\right) F\left(t^{\prime}\right) d t^{\prime}
$$

Multiplicando a (2.28) por $v(0)$, usando (2.27) y suponiendo que el principio de equipartición se satisface para el estado inicial $\left(\left\langle v^{2}(0)\right\rangle=k_{B} T M^{-1}\right)$ obtenemos como resultado

$$
\langle v(t) v(0)\rangle=\frac{k_{B} T}{M} \exp (-\gamma t / M),
$$


lo cual nos dice que la función de autocorrelación de la velocidad decae exponencialmente en un tiempo característico $\tau=M / \gamma$ que depende de la masa de la partícula y de las propiedades del fluido. Cabe mencionar que esta $\tau$ es idéntica a la $\tau_{1}$ de la descripción de Langevin y a la $\tau$, con la que identificamos a la ecuación de Langevin con un proceso de Ornstein-Uhlenbeck, por lo que a partir de este momento adoptamos a $\tau$ como nomenclatura general. Además, la forma exponencial (2.29) de la autocorrelación de la velocidad es la propiedad más conocida que caracteriza a un proceso Markoffiano.

Por otra parte, si a partir de (2.28) calculamos $v^{2}(t)$ y promediamos, encontramos, usando además las ecuaciones (2.26), (2.27) y (2.29), que

$$
\frac{M}{2}\left\langle v^{2}(t)\right\rangle=\frac{1}{2} k_{B} T \exp (-2 \gamma t / M)+\frac{B}{2 \gamma}[1-\exp (-2 \gamma t / M)] .
$$

Puesto que se espera físicamente que el principio de equipartición tenga validez asintótica (esto es, para $t \gg \tau$ ) tenemos que, en ausencia de perturbaciones externas, el lado derecho de esta última ecuación debe de ser igual a $k_{B} T / 2$. Entonces $B$ tiene que tener un valor muy específico, lo que nos lleva al bien conocido teorema de fluctuación-disipación

$$
B=\gamma\left(k_{B} T\right)
$$

Esta ecuación establece el hecho de que la intensidad de la fuerza fluctuante, cuantificada a través de $B$, está relacionada a la disipación de la energía de la partícula Browniana debida al fluido de tal manera que, si uno conoce la primera, la segunda se puede calcular, y recíprocamente. Es en este sentido que las fuerzas sisternática y fluctuante están relacionadas, como ya habíamos mencionado antes. Nótese también que las ecuaciones (2.26) y (2.31) sugieren que, en general,

$$
\gamma=\frac{1}{k_{B} T} \int_{0}^{\infty}\langle F(t) F(0)\rangle d t
$$

donde hemos utilizado la propiedad de que $\delta(t)$ es una función par en $t$. La Ec. (2.32) ofrece una forma de calcular el coeficiente de fricción $\gamma$ a partir del conocimiento de la función de autocorrelación de la fuerza fluctuante $F(t)$.

Por otro lado, calculando la posición de la partícula Browniana a partir de (2.22) se tiene que el desplazamiento cuadrático medio se puede escribir como

$$
\left\langle[x(t)-x(0)]^{2}\right\rangle=\int_{0}^{t} d t^{\prime} \int_{0}^{t} d t^{\prime \prime}\left\langle v\left(t^{\prime}\right) v\left(t^{\prime \prime}\right)\right\rangle
$$


el cual nos permite, utilizando la relación de Einstein, definir al coeficiente de difusión $D$ en términos de la autocorrelación de la velocidad como

$$
D=\int_{0}^{\infty}\langle v(0) v(t)\rangle d t
$$

Una sustitución directa de (2.29) en (2.34) nos da como resultado, después de una integración elemental, la relación de Einstein (2.18) escrita en términos del coeficiente de fricción,

$$
D=\left(k_{\beta} T\right) \gamma^{-1}
$$

Si aceptamos a (2.32) como una relación válida entre el coeficiente de fricción macroscópico $\gamma$ y las fluctuaciones microscópicas contenidas en $F(t)$, la relación (2.35) nos muestra cómo el coeficiente de transporte de nuestro sistema, la partícula Browniana, también se relaciona a las fluctuaciones microscópicas.

Obsérvese que todos los resultados obteniclos hasta ahora son válidos para $t \gg \tau$. Una descripción valida para todo tiempo también se puede obtener a partir de (2.28), como a continuación mostraremos.

Si escribimos a esta solución como

$$
v(t)-v(0) \exp (-\gamma t / M)=\frac{1}{M} \int_{0}^{t} \exp \left(-\gamma\left(t-t^{\prime}\right) / M\right) F\left(t^{\prime}\right) d t^{\prime}
$$

podemos inferir que las propiedades estadisticas del lado izquierdo son las mismas que las del lado derecho. Pero éste contiene a $F(t)$ linealmente; esto es, tenemos una suma de variables dependientes de la fuerza estocástica $y$, por lo tanto, estocásticas ellas mismas. Si $F(t)$ es una variable Gaussiana, entonces el lado derecho será también una variable Gaussiana [18]. Por lo tanto la combinación $v(t)-v(0) \exp (-\gamma t / M)$ está distribuida Gaussianamente. La distribución de velocidades puede escribirse entonces como

$$
\begin{gathered}
f_{v}(v, t ; v(0))=\left(\frac{M}{2 \pi k_{B} T[1-\exp (-2 \gamma t / M)]}\right)^{1 / 2} \\
\times \exp \left(-\frac{M[v(t)-v(0) \exp (-\gamma t / M)]^{2}}{2 k_{B} T[1-\exp (-2 \gamma t / M)]}\right)
\end{gathered}
$$

Para el caso $t \gg \tau$ esta expresión se reduce a

$$
f_{v}(v, t ; v(0)) \simeq\left(\frac{M}{2 \pi k_{g} T}\right)^{\frac{1}{2}} \exp \left(-\frac{M}{2 k_{B} T} v^{2}\right)
$$


la cual es precisamente una distribución Maxwelliana de velocidades que describe al estado de equilibrio. Podemos decir entonces que la partícula Browniana llega, después de cierto tiempo, a un estado de equilibrio independientemente de la velocidad $v(0)$ que haya tenido inicialmente.

De la solución (2.36) se puede encontrar también la distribución de posiciones $x$ al tiempo $t$. El resultado es [18]

$$
\begin{gathered}
f_{x}(x, t ; x(0), v(0))=\left(\frac{M \tau^{-2}}{2 \pi k_{B} T\left[2 \tau^{-1} t-3+4 \exp (-\gamma t / M)-\exp (-2 \gamma t / M)\right]}\right)^{1 / 2} \\
\quad \times \exp \left(-\frac{M \tau^{-2}[x-x(0)-\tau v(0)\{1-\exp (-\gamma t / M)\}]^{2}}{2 k_{B} T\left[2 \tau^{-1} t-3+4 \exp (-\gamma t / M)-\exp (-2 \gamma t / M)\right]}\right) \cdot(2.39)
\end{gathered}
$$

De igual manera, para tiempos $t \gg \tau$ la expresión anterior se reduce a

$$
f_{x}(x, t ; x(0), v(0)) \simeq \frac{1}{(4 \pi D t)^{1 / 2}} \exp \left(-\frac{[x-x(0)]^{2}}{4 D t}\right),
$$

donde se ha usado la definición (2.35) para $D$. Es claro entonces que en este régimen temporal se recupera la densidad numérica (2.9) obtenida por el método de Einstein.

Volviendo a la distribución (2.39) podemos obtener la expresión general del desplazamiento cuadrático medio para cualquier régimen temporal calculando

$$
\left\langle[x(t)-x(0)]^{2}\right\rangle=\int_{0}^{\infty} x^{2} f_{x}(x, t ; x(0), v(0)) d x
$$

y utilizando una vez más el principio de equipartición. El resultado final de este cálculo es

$$
\left\langle[x(t)-x(0)]^{2}\right\rangle=\frac{2 k_{B} T}{M \tau^{-2}}\left[\frac{\gamma t}{M}-1+\exp \left(-\frac{\gamma t}{M}\right)\right],
$$

del cual se obtiene

$$
\left\langle[x(t)-x(0)]^{2}\right\rangle=\left\{\begin{array}{lll}
2 D t & \text { si } & t \gg \tau \\
\left(k_{B} T M^{-1}\right) t^{2} & \text { si } & t \ll \tau .
\end{array}\right.
$$

El primero de estos resultados no es otro que la relación de difusión de Einstein, mientras que el segundo tiene un significado físico muy claro: para tiempos pequeños el efecto del medio circundante sobre la partícula Browniana es despreciable, por lo que ésta se mueve como partícula libre. 


\subsubsection{Ecuación de Fokker-Planck}

Una manera diferente de analizar el comportamiento de una partícula Browniana es establecer directamente una ecuación que satisfaga la función de densidad de probabilidad $f_{v}$ estudiada al final del apartado anterior. Es importante hacer notar que en el método de la ecuación de Langevin se construye la función $f_{v}$. En particular, si se supone que $f_{v}$ es una distribución Gaussiana, el proceso de construcción es relativamente sencillo de llevar a cabo, como ya se mostró. Sin embargo, si el proceso dinámico que se está estudiando es tal que la FDP no es Gaussiana se presentan dificultades que en general son muy difíciles de superar con el método de la ecuación de Langevin. Otra situación en la que es útil trabajar directamente con la función de distribución se presenta cuando se imponen restricciones adicionales al problema. Si estas restricciones pueden expresarse en la forma de condiciones de frontera, entonces la reducción a un problema de ecuaciones diferenciales parciales con condiciones de frontera nos proporciona un método más directo de obtener la solución necesaria.

Como ya habíamos mencionado antes, la velocidad de la partícula Browniana es un proceso Markoffiano, lo que implica que la función de densidad de probabilidad $f_{v}$ satisface lo que se conoce como una ecuación maestra que se escribe corno

$$
f_{v}(v, t+\Delta t)=\int f_{v}(v-\Delta v, t) W(v-\Delta v ; \Delta v) d(\Delta v),
$$

donde $W(v ; \Delta v)$ es la probabilidad de transición de que $v$ experimente un incremento $\Delta v$ en el intervalo $\Delta t$. Entonces puede demostrarse [18], a partir de (2.44), que $f_{v}(v, t ; v(0))$ satisface una ecuación de Fokker-Planck cuya forma explíita está dada por

$$
\frac{\partial f_{v}}{\partial t}=\gamma \frac{\partial}{\partial v}\left(v f_{v}+\frac{k_{B} T}{M} \frac{\partial f_{v}}{\partial v}\right)
$$

La solución a esta ecuación diferencial parcial se puede obtener utilizando métodos bien conocidas [18] para unas condiciones iniciales dadas y puede además mostrarse que, para tiempos grandes,

$$
f_{v}(v, t ; v(0)) \rightarrow f_{v}^{e q}(v),
$$

donde $f_{v}^{e q}(v)$ es la distribución Maxwelliana de velocidades para la partícula con velocidad $v$, masa $M$, iumersa en un fluido a temperatura $T$ y cuya forma explícita está dada por la ecuación (2.38) de la sección anterior. Por lo tanto, podemos concluir que los esquemas de Langevin y de Fokker-Planck son equivalentes. 


\subsection{Modelos Microscópicos}

Como ya hemos tenido oportunidad de ver, la teoría fenomenológica del movimiento Browniano proporciona una descripción bastante satisfactoria de las observaciones experimentales. Sin embargo, es una teoría esencialmente incompleta (como toda teoría fenomenológica), pues tanto el coeficiente de fricción como el de difusión tienen que obtenerse a partir de datos experimentales. Una descripción más fundamental tendría que ser estrictamente microscópica y en ella los coeficientes antes mencionados deberían de poderse calcular a partir de los parámetros moleculares del fluido. Para alcanzar este objetivo, en los primeros trabajos la metodología consistió en partir de una descripción mesoscópica, a partir de la cual se obtiene la ecuación de evolución para la función de distribución de una partícula pesada. Posteriormente, y utilizando diversas técnicas tales como los operadores de proyección, fue posible derivar formalmente la ecuación de Langevin. Sin embargo, en todos estos enfoques se obtienen expresiones meramente formales para los coeficientes de transporte que no es posible evaluar de manera explícita. Es por ello que ha sido necesario recurrir a simulaciones de computadora para realizar cálculos explícitos de los mismos. Sólo en algunos casos muy específicos (esferas duras) ha sido posible algún desarrollo analítico, pero aún así los cálculos numericos son necesarios para extraer la información relevante de las expresiones resultantes, como a continuación veremos.

\subsubsection{Modelo de Fluido: Primeros Trabajos}

La primera teoría microscópica del movimiento Browniano fue desarrollada por Kirkwood [19] y es aplicable a la autodifusión de un átomo o molécula inmersa en un medio compuesto de partículas idénticas, tales como el argón líquido. Este tipo de sistemas están descritos, a nivel microscópico, por un Hamiltoniano de la forma

$$
H=\sum_{i=1}^{N} \frac{p_{i}^{2}}{2 m}+\sum_{i<j}^{N} \Phi\left(r_{i j}\right)
$$

donde $\mathbf{p}_{i}$ y $m$ son el momento y la masa de la $i$-ésima partícula del fluido, mientras que $\Phi\left(r_{i j}\right)$ es el potencial de interacción entre pares de partículas y que sólo depende de la distancia $r_{i j}$ entre ellas. El principal resultado de esta teoría es una expresión para el coeficiente de fricción $\gamma$ en términos de una función de 
autocorrelación de la forma

$$
\gamma=\frac{1}{3 k_{i} T} \int_{0}^{T_{k}}\langle\mathrm{~F}(t) \cdot \mathbf{F}(0)\rangle d i
$$

donde $T$ es la temperatura en equilibrio del fluido, $F(t)$ es la fuerza total al tiempo $t$ actuando sobre una cierta partícula del fluido debida a todas las demás y el promedio $\langle\ldots\rangle$ se toma con respecto a la función de distribución de equilibrio del fluido. El tiempo $\tau_{k}$ fue introducido por Kirkwood debido a que la integral en (2.48) se anula en el límite $\tau_{k} \rightarrow \infty$ para sistemas con un número finito de grados de libertad ${ }^{4}$. Esto implica que los tiempos de observación deben de ser grandes respecto a los tiempos característicos asociados a las variables microscopicas, pero pequeños relativos a los tiempos de recurrencia del sistema. Aunque lo anterior establece un rango de validez para los valores de $\tau_{k}$, no se ha encontrado la manera de calcular este tiempo de forma analitica. Ahora bien, aunque Kirkwood arguyó de manera plausible que (2.48) es válida para calcular el coeficiente de fricción de una partícula que tenga una masa mayor que las del fluido circundante, esta. suposición sólo pudo estudiarse en trabajos posteriores.

El primer análisis riguroso de un sistema compuesto por una partícula pesada de rnasa $M$ interaccionando con un fluido compuesto de partículas idénticas de masa $m$ fue debido a Lebowitz y Rubin [20]. Estos autores plantearon el siguiente Hamiltoniano,

$$
H=\frac{P^{2}}{2 M}+\left[\sum_{i=1}^{N} \frac{p_{i}^{2}}{2 m}+\sum_{i<j}^{N} \Phi\left(r_{i j}\right)\right]+\sum_{i=1}^{N} U\left(\left|\mathbf{r}_{i}-\mathbf{R}\right|\right)
$$

donde $\mathbf{r}_{\boldsymbol{i}}$ y $\mathbf{p}_{\boldsymbol{i}} \equiv m \mathbf{v}_{\boldsymbol{i}}$ son las posiciones y momentos de la $i$-ésima partícula del fluido, mientras que $\mathbf{R}$ y $\mathbf{P} \equiv M \mathrm{~V}$ son la posición y el momento de la partícula pesada, respectivamente; $\Phi$ corresponde a la interacción entre cualquier par de partículas del fluido y $U$ es la energía potencial de interacción entre éstas y la partícula masiva. Si se considera además el efecto de un campo externo

\footnotetext{
${ }^{4}$ Este hecho es una consecuencia del teorema de recurrencia de Poincaré, el cual establece que, para un sistema mecánico en el cual las fuerzas dependan sólo de las coordenadas espaciales, la trayectoria en el espacio fase regresa a una vecindad arbitrariamente pequeña del punto inicial si el sistema tiene un número finito de grados de libertad y se espera un tiempo lo suficientemente grande (Ver apéndice $V$ de la Ref. [18] para una demostración). El tiempo de recurrencia (o ciclo de Poincaré) designa el tiempo que tarda el sistema en regresar a la vecindad del punto inicial en este tipo de movimiento "cuasi-periódico". Aunque este tiempo es extraordinariamente grande para sistemas reales de moléculas, es importante tenerlo presente para desarrollos posteriores.
} 
constante $\mathbf{E}$ tenemos que la evolución temporal de la densidad de probabilidad $f^{(\mathcal{N})}\left(\mathbf{R}, \mathbf{P}, \mathbf{r}^{N}, \mathbf{p}^{N} ; t\right)$ para este sistema de $\mathcal{N}=N+1$ partículas está gobernada por la ecuación de Liouville

$$
\frac{\partial f^{(\mathcal{N})}}{\partial t}=-\mathrm{i} \mathcal{L} f^{(\mathcal{N})}-\mathbf{E} \cdot \frac{\partial f^{(\mathcal{N})}}{\partial \mathbf{P}},
$$

donde $\mathcal{L}$ es el correspondiente operador de Liouville. La función de distribución de la partícula pesada

$$
f(\mathbf{R}, \mathbf{P} ; t)==\int f^{(\mathcal{N})}\left(\mathbf{R}, \mathbf{P}, \mathbf{r}^{N}, \mathbf{p}^{N} ; t\right) d \mathbf{r}^{N} d \mathbf{p}^{N}
$$

obedece una ecuación de movimiento que se obtiene al integrar (2.50) sobre $\mathbf{r}^{N}$ y $\mathrm{p}^{N}$. Si a continuación se realiza un desarrollo en serie de potencias del cociente de masas $\lambda^{2}=m / M$ :

$$
\begin{aligned}
f^{(\mathcal{N})} & =\lambda f_{1}^{(\mathcal{N})}+\lambda^{2} f_{2}^{(\mathcal{N})}+\ldots \\
f & =\lambda f_{1}+\lambda^{2} f_{2}+\ldots
\end{aligned}
$$

entonces se obtiene, a primer orden en el desarrollo anterior, la ecuación de FokkerPlanck para la función de distribución de la partícula pesada de la forma

$$
\frac{\partial f}{\partial t}=\gamma \frac{\partial}{\partial \mathbf{P}} \cdot\left(\mathbf{P} f+M k_{B} T \frac{\partial f}{\partial \mathbf{P}}\right)-\frac{\mathbf{P}}{M} \cdot \frac{\partial f}{\partial \mathbf{R}}-\mathbf{E} \cdot \frac{\partial f}{\partial \mathbf{P}},
$$

siendo esta ecuación válida para tiempos muy grandes comparados con los tiempos de relajación característicos del fluido. Además, como en la derivación de (2.52) se utilizó el límite termodinámico $N \rightarrow \infty$, entonces se puede reescribir la expresión de Kirkwood para el coeficiente de fricción $\gamma$ en el límite $\lambda^{2} \ll 1$ como

$$
\gamma=\frac{1}{3 k_{B} T} \int_{0}^{\infty} \lim _{\lambda^{2} \rightarrow 0}\langle\mathbf{F}(t) \cdot \mathbf{F}(0)\rangle d t,
$$

donde la función de autocorrelación de la fuerza tiene que ser evaluada en un sistema de referencia en el cual la partícula pesada este fija en el espacio. Posteriormente Lebowitz y Résibois [21] extendieron el análisis anterior para el caso de un campo externo dependiente del tiempo $\mathbf{E} \mathrm{e}^{\mathrm{i} \omega t}$ actuando sobre la partícula pesada. Utilizando un operador que promedia sobre las variables del fluido mostraron además la equivalencia de sus resultados con aquellos obtenidos en el trabajo anterior de Résibois y Davis [22]. 
En todos los trabajos anteriores los términos de orden superior en $\lambda$ son despreciados para poder así reducir las ecuaciones de evolución mesoscópicas a las ecuaciones que describen al movimiento Browniano macroscópico. Sin embargo, la dependencia temporal de estos térninos de orden superior no fue suficientemente investigada como para justificar su eliminación para tiempos muy grandes. Mazur y Oppenheim [23] fueron al parecer los primeros en estudiar este problema trabajando directamente con la ecuación de Langevin para la partícula pesada en lugar de la función de distribución, como en los casos anteriores. Su metodología consistió en construir la ecuación dinámica para el momento $\mathbf{P}$ de la partícula pesada a partir del Hamiltoniano (2.49) para posteriormente estudiar bajo qué condiciones se reduce a la ecuación de Langevin fenomenológica. Estos autores encontraron una ecuación tipo Langevin que es válida hasta $O\left(\lambda^{2}\right)$ para cualquier escala de tiempo, suponiendo que el momento de la partícula pesada sea de orden $\lambda^{-1}$ y que las funciones de correlación de las variables asociadas al fluido en presencia de una partícula pesada fija en el espacio decaigan con suficiente rapidez. Ahora bien, en el límite $\lambda^{2} \rightarrow 0, t \rightarrow \infty, \lambda^{2} t=c t e$. (conocido en la literatura como límite de acoplamiento débil) se recupera la ecuación de Langevin fenomenológica junto con la expresión (2.48) que ahora se escribe como

$$
\gamma=\frac{1}{3 k_{B} T} \int_{0}^{\infty} \lim _{\substack{\lambda^{2} \rightarrow 0 \\ t \rightarrow \infty \\ \lambda^{2} t=c t e}}\langle\mathbf{F}(t) \cdot \mathbf{F}(0)\rangle d t
$$

En este punto de nuestra exposición es importante mencionar que, aunque la fuerza microscópica $F(t)$ se comporta como una fuerza estocástica en esta aproximación, su estructura (al igual que en todos los casos anteriores) es tan complicada que no es posible hacer un cálculo explícito del coeficiente de fricción.

Puesto que el momento de la partícula masiva es de orden $\lambda^{-1}$, se puede inferir que la escala de tiempo en que la función de distribución $f(\mathbf{R}, \mathbf{P} ; t)$ experimenta variaciones significativas es lenta comparada con la correspondiente escala de tiempo asociada al fluido. Esto implica que puede considerarse al momento de la partícula pesada como una variable lenta en comparación con los momentos de las partículas del fluido, lo cual está demostrado explícitamente que se cumple en el límite $\lambda^{2} \rightarrow 0$ [24]. Ahora bien, como a nivel macroscópico la ecuación de Langevin está definida solamente en términos del momento de la partícula masiva, es razonable suponer que, a nivel mesoscópico, la variable dinámica que describa al estado del sistema será una coordenada de momento asociada a la partícula pesada y que tome en cuenta el efecto de todas las demás variables del sistema. 
Esto es,

$$
P(t) \rightarrow \widehat{P}\left(t ; \mathbf{r}^{N}, \mathbf{p}^{N}\right) \equiv \hat{P}(t)
$$

Es sabido que [25], en términos de la nueva variable $\widehat{P}(t)$, resulta una descripción totalmente análoga a la obtenida con (2.50) si ahora tomamos como punto de partida la ecuación de evolución para $\hat{P}(t)$, la cual tiene la forma

$$
\frac{\partial \hat{P}(t)}{\partial t}=\mathrm{i} \mathcal{L} \hat{P}(t)
$$

Utilizando la técnica de los operadores de proyección sugerida por Zwanzig [26] y desarrollada posteriormente por Mori [27], se puede reescribir la Ec. (2.56) de la forma conocida en la literatura como ecuación generalizada de Langevin y que es la siguiente

$$
\frac{d \widehat{P}(t)}{d t}=-\int_{0}^{t} \widehat{K}\left(t-t^{\prime}\right) \widehat{P}\left(t^{\prime}\right) d t^{\prime}+\widehat{F}(t)
$$

donde

$$
\widehat{K}\left(t-t^{\prime}\right)=\left\langle\widehat{F}(t) \hat{F}\left(t^{\prime}\right)\right\rangle\left\langle\widehat{P}^{2}(0)\right\rangle^{-1}
$$

y

$$
\hat{F}(t)=\exp [t(1-\mathcal{P}) \mathrm{i} \mathcal{L}](1-\mathcal{P}) \mathrm{i} \mathcal{L} \hat{P}(0)
$$

son conocidas como función de memoria y fuerza aleatoria, respectivamente, mientras que $\mathcal{P}$ es el operador de proyección sobre la variable $P(0)$. Obsérvese que (2.57) es una ecuación formal de evolución para la variable $\widehat{P}(t)$ en la que el efecto del resto de las variables del sistema está contenido en $\hat{F}(t)$, por lo que, en principio, es una ecuación exacta a nivel mesoscópico. Sin embargo, su estructura es tan complicada que obtener analíticamente resultados cuantitativos con ella es una tarea por demás imposible. Muestra de ello es que, en principio, se puede calcular el coeficiente de difusión $D$ a partir de la integral de la función de autocorrelación del momento $\widehat{P}(t)$. Esta función puede a su vez obtenerse a partir de (2.57) si multiplicamos a esta última por $\widehat{P}\left(t^{\prime}\right)$ y tomamos el promedio estadístico, lo que resulta en una ecuación para la función de autocorrelación del momento que puede resolverse formalmente utilizando la transformada de Laplace. El resultado queda expresado en términos de la función de memoria, misma que puede representarse por medio de un desarrollo en fracciones continuas [28]. Sin embargo, los coeficientes de este desarrollo (conocidos como coeficientes de Mori) 
no pueden calcularse analíticamente; solo pueden obtenerse numéricamente a partir de simulaciones de dinámica molecular, to cual ha sido mostrado en forma explícita para un fluido de Lennard-Jones [29].

La suposición (2.55) se conoce como un granulamiento grueso y corresponde a una contracción de la información necesaria para describir el estado del sistema. Aunque el procedimiento para realizar este granulamiento grueso es perfectamente natural para el caso del movimiento Browniano $\left(\lambda^{2} \rightarrow 0\right.$, como ya habíamos mencionado), no deja de ser una aproximación mesoscópica, por lo que mucha de la información microscópica contenida en el Hamiltoniano (2.49) se pierde. Este es un punto importante porque, en principio, no se sabe cuánta de esta información es relevante para recuperar la fenomenología macroscópica. Sin embargo, podría suponerse que, si se toma un conjunto suficientemente grande de variables gruesas, el estado del sistema estará bien definido, pudiéndose obtener entonces las ecuaciones de transporte macroscópicas que obedecen los promedios de estas variables. Este esquema general fue propuesto por M. Green [30] y de hecho es el fundamento de la aplicación del método de proyectores a otro tipo de sistemas. Pero, para el caso específico del movimiento Browniano, se ha mostrado que, si no se considera a la familia completa de formas multilineales de $\mathbf{P}(\mathbf{P P}, \mathbf{P P P}, \ldots$ ) co mo variables lentas, entonces la función de autocorrelación del momento muestra un comportamiento asintótico no-exponencial [31], observado por primera vez en simulaciones de dinámica molecular para fluidos de esferas duras [32\} y posteriormente en fluidos con potenciales continuos [33]. Ahora bien, dentro del esquema propuesto por Green este problema surge debido al granulamiento grueso que se está usando para obtener el nivel mesoscópico de descripción; en este nivel el problema se explica en términos de una interacción de tipo hidrodinámico entre las partículas del fluido. Esta explicación, sin embargo, no elimina la posibilidad de que este efecto (conocido como de "cola larga") tenga un origen en la dinámica microscópica del sistema, como ya habíamos antes mencionado. Por lo tanto, todos estos resultados, en cierta forma, ponen en evidencia las limitaciones de este tipo de enfoque ${ }^{5}$.

\footnotetext{
${ }^{5}$ El problema de las "colas largas" en las funciones de autocorrelación ha sido muy debatido en la literatura pero, como su relevancia para el presente trabajo es marginal, no lo abordaremos en detalle. Pará una revisión de este tema pueden consultarse las referencias [34] y [35], las cuales ofrecen una amplia perspectiva desde puntos de vista distintos pero complementarios.
} 


\subsubsection{Modelo de Fluido: Desarrollos Posteriores}

Como hemos podido ver, el problema del cálculo de los coeficientes de transporte consiste, esencialmente, en resolver un problema de muchos cuerpos, lo que resulta analíticamente imposible en general para sistemas descritos por el Hamiltoniano (2.48). Sin embargo, utilizando técnicas de dinámica molecular es posible realizar el cálculo numéricamente, por lo que debe de plantearse una forma explícita para el potencial de interacción. Siguiendo esta metodologia Vogelsang y Hoheisel [36] fueron los primeros en calcular, para un potencial de Lennard-Jones (12-6), el coeficiente de fricción $\gamma$ a partir de la expresión (2.48), encontrándose que la integral de la función de autocorrelación de la fuerza alcanza un valor constante para el caso de una partícula masiva con una masa tal que $50 \leq M \leq 180$ e inmersa en un fluido compuesto de entre 256 y 864 partículas livianas. Para los valores de masa utilizados el tiempo $\tau_{k}$, postulado por Kirkwood y que necesariamente debe de ser tomado en cuenta para el caso de sistemas finitos, resulta tener un valor de entre 0.75 y 2.0 ps. Estos autores mostraron que el cálculo de $\gamma$ a partir de la función de autocorrelación de la fuerza, para el caso de un fluido compuesto de partículas idénticas, sólo puede realizarse utilizando la ecuación generalizada de Langevin (2.57). Adicionalmente, se encuentran resultados totalmente congruentes con los anteriores si además de variar la masa también se varía el volumen de la partícula masiva [37].

Para el caso de un fluido de esferas duras elásticas ha sido posible obtener de manera analítica, adaptando el método de las escalas múltiples de tiempo [38] al caso de potenciales discontinuos, una expresión explícita y exacta para el coeficiente de fricción $\gamma$ válida en el límite $\lambda^{2} \rightarrow 0$ [39]. Este coeficiente se separa naturalmente en la suma de dos contribuciones. La primera resulta ser proporcional a la densidad de equilibrio del fluido en contacto con la partícula pesada y corresponde a las predicciones de la aproximación cinética de Enskog. El segundo término proviene del ritmo instantáneo de transferencia de momento del fluido a la partícula masiva y corresponde esencialmente a la expresión de Kirkwood (2.48) para el caso del potencial de esfera dura. Aunque se encuentra una expresión explícita para la fuerza $\mathbf{F}(t)$ ejercida por las partículas del fluido durante las colisiones con la partícula pesada, el cálculo "exacto" de este término sólo puede realizarse por medio de simulaciones de dinámica molecular [40], del mismo modo que con el caso del potencial continuo de Lennard-Jones. Los resultados obtenidos con estos modelos muestran claramente que es perfectamente posible calcular los 
coeficientes de transporte macroscópicos, aunque sólo para casos particulares y aceptando las limitaciones propias de los métodos de la dinámica molecular.....

\subsection{Conclusiones}

Tomando en cuenta todo lo anteriormente dicho en este capítulo, podemos sacar algunas conclusiones generales. En primer lugar, vemos que la fenomenología del movimiento Browniano tiene el estatus de teoría satisfactoriamente desarrollada y con múltiples aplicaciones en las más diversas áreas de investigación, tanto teóricas como aplicadas. Sin embargo, no se puede decir que su fundamentación microscópica esté totalmente acabada. Aunque, como ya hemos tenido ocasión de ver, se ha podido encontrar la función de distribución para la partícula pesada y su ecuación de evolución, sólo se han podido encontrar expresiones formales para el coeficiente de fricción. De estas expresiones no se encuentra indicación alguna de cómo calcularlas explícitamente para una interacción específica, salvo para el caso de esferas duras, como ya también se indicó. Debido a esta falta de resultados cuantitativos es que todavía son de gran utilidad los enfoques termodinámicos (fenomenológicos) a este problema [41].

Un gran avance sería el poder tener un modelo en el que pudiera llevarse a cabo de forma completa el siguiente programa, que consiste en:

- Resolver las ecuaciones de movimiento del sistema mecánico consistente en una partícula pesada acoplada a un conjunto de partículas livianas que constituyen el "medio" en el que aquella está sumergida. La solución consiste de expresiones para las coordenadas y momentos al tiempo $t$ en términos de condiciones iniciales para cada una de las partículas del sistema.

- Suponer que los valores de las coordenadas y momentos iniciales del "medio" satisfacen una distribución de equilibrio bien definida, tal como la que describe a un ensamble canónico. Las partículas livianas juegan entonces el papel de "baño térmico".

- Mostrar que las coordenadas de posición y momento de la partícula pesada, como funciones del tiempo, representan a un proceso estocástico del tipo descrito por la teoría fenomenológica estudiada en el presente capítulo.

No hay duda de que éste es un programa nuy ambicioso y que no ha podido llevarse a cabo de manera completa para el modelo de fluido descrito por el Ha- 
miltoniano (2.49). Sin embargo, existe una clase de modelos para los cuales es posible completar el programa anterior de manera satisfactoria. Estos son los así llamados modelos de osciladores armónicos acoplados. Son lo suficientemente simples como para tratarlos de forma exacta y mantienen el suficiente contenido físico como para ser de interés. Su descripción será dada en el capítulo siguiente. 


\section{Capítulo 3}

\section{Modelos de Osciladores Acoplados}

\subsection{Introducción}

En el capítulo 2 vimos que la principal dificultad para formalizar una descripción microscópica del movimiento Browniano en un fluido es la gran cantidad de aproximaciones que hay que hacer para poder llegar a la ecuación de Fokker-Planck o de Langevin. Debido a lo anterior se vuelve muy difícil poder determinar si los resultados obtenidos son producto de la dinámica intrínseca del modelo o de las aproximaciones utilizadas para llegar al nivel de descripción macroscópico. Esto es particularmente importante para el caso de resultados de difícil interpretación, tales como las "colas largas" en las funciones de autocorrelación. Todos estos factores sugieren una posible alternativa: plantear un modelo diferente que sea mucho más sencillo en su estructura matemática, pero que mantenga suficiente contenido físico como para seguir siendo de interés.

Los modelos llamados genéricamente de osciladores armónicos acoplados han podido satisfacer estos requerimientos. Son de los sistemas de muchos cuerpos más sencillos que existen, puesto que su dinámica se conoce de manera exacta. Esto hace que su comportamiento estadístico se haya podido investigar exhaustivamente, empezando con el trabajo de Mazur y Montroll para el caso de un sistema de osciladores con masas idénticas [42]. Es importante mencionar, sin embargo, que este tipo de sistemas también tienen sus limitaciones. La más seria de ellas es que su mecanismo de transporte de energía no satisface la ley fenomenológica de Fourier [43]. Pero como este último problema es irrelevante para el estudio del 
movimiento Browniano, podemos ignorarlo sin detrimento de desarrollos posteriores. Adernás, en los límites adecuados, estos sistemas satisfacen los requerimentos físicos que son relevantes en el estudio del movimiento Browniano, por lo que los adoptaremos como punto de partida de nuestra investigación.

Empezaremos haciendo una revisión de los modelos de osciladores acoplados con interacciones a primeros vecinos. Veremos que en este tipo particular de modelos se puede determinar un cierto régimen temporal en el que ocurre movimiento Browniano, recuperándose tanto la ecuación de Langevin como practicamente todos los resultados fenomenológicos presentados en el capítulo anterior. A continuación complementaremos nuestra discusión con la revisión de los modelos con interacciones de largo alcance. Finalmente concluiremos con una revisión de los factores que hacen plausible el uso de modelos de osciladores para el estudio del movimiento Browniano.

\subsection{Interacciones a Primeros Vecinos}

El modelo dinámico que vamos a estudiar es una cadena unidimensional compuesta de $N+1$ partículas cuyas posiciones de equilibrio están separadas por una distancia $a=1$. A continuacion emumeramos las partículas desde $-N / 2$ hasta $N / 2$ y escogemos la masa de todas ellas igual a $m$ con excepción de la partícula central, que tiene masa $M$. Esto equivale a decir que $M_{i}=m$ si $i \neq 0$ y $M_{0}=M$, con la condición $M>m$. Además suponemos que sólo hay interacciones armónicas entre primeros vecinos, todas iguales y cuantificadas por una constante de fuerza $\kappa$. El Hamiltoniano de nuestro sistema se escribe entonces como

$$
H=\frac{1}{2}\left[\sum_{i=-N / 2}^{N / 2} \frac{P_{i}^{2}}{M_{i}}+\kappa \sum_{i=-N / 2}^{N / 2}\left(X_{i+1}-X_{i}\right)^{2}\right],
$$

donde $X_{i}$ es el desplazamiento de la $i$-ésima particula desde su posición de equilibrio y $P_{i}$ es el correspondiente momento conjugado. Se han supuesto además condiciones de frontera periódicas; esto es

$$
X_{(N / 2)+1} \equiv X_{-N / 2}
$$

En comparación con el Hamiltoniano (2.49) del modelo de fluido, (3.1) tiene una estructura mucho más sencilia. En primer lugar se está considerando sólo el caso unidimensional; las partículas ya no pueden moverse en todo el espacio, sino 
que ahora están constreñidas a oscilar longitudinalmente alrededor de unas posiciones de equilibrio definidas por una estructura espacial periódica. Por último, las interacciones son armónicas y se restringen a primeros vecinos. Todas estas características hacen que nuesto sistema sea un modelo de un sólido cristalino muy simplificado. De hecho, en el lenguaje del estado sólido lo que tenemos es una red cristalina perfecta perturbada por un defecto (impureza) localizado en el sitio 0 . Ahora bien, aunque con este modelo no es posible estudiar caracteristicas propias de un fluido, se sigue teniendo a un "sistema" (partícula pesada) que interactúa con un "baño térmico" (la red cristalina). Además, si recordamos que muchas sustancias (como por ejemplo el argón) se solidifican en una red cristalina, vemos que el modelo sigue teniendo relevancia para el estudio de sustancias reales. Las partículas de masa $m$ juegan ahora el papel del "Gluido", mientras que el defecto corresponde a la partícula Browniana.

Las ecuaciones de movimiento de nuestro sistema, que se obtienen a partir del Hamiltoniano (3.1), tienen la forma explícita

$$
\frac{d^{2} X_{i}}{d t^{2}}=\frac{\kappa}{m}\left(1-\frac{Q}{Q+1} \delta_{i 0}\right)\left(X_{i+1}+X_{i-1}-2 X_{i}\right)
$$

para $i=-N / 2, \ldots, N / 2$, siendo $Q=M / m-1$. La solución de este sistema es inmediata y se puede escribir en forma exacta como una superposición de funciones periódicas, como veremos a continuación. Esto le permitió a Rubin [44], en el primer tratamiento formal del tema, estudiar de manera exhaustiva el movimiento de una impureza muy pesada inmersa en una cadena infinita de osciladores armónicos, encontrando una similitud muy grande con el de una partícula Browniana unidimensional libre de fuerzas externas. Con esta comprobación a posteriori queda confirmada la plausibilidad del Hamiltoniano (3.1) como un modelo viable para el estudio del movimiento Browniano.

Las soluciones $\left\{X_{i}\right\}$ a (3.3) y los respectivos momentos $\left\{P_{i}\right\}$ se pueden escribir como

$$
\begin{aligned}
X_{i}(t) & =\sum_{j}\left[A_{i j}(t) X_{j}+B_{i j}(t) P_{j}\right] \\
P_{i}(t) & =\sum_{j}\left[C_{i j}(t) X_{j}+D_{i j}(t) P_{j}\right]
\end{aligned}
$$

donde $A, B, C$ y $D$ son funciones del tiempo que se describirán más adelante, mientras que $X_{j} \equiv X_{j}(0)$ y $P_{j} \equiv P_{j}(0)$ son las condiciones iniciales del sistema. 
A partir de (3.5) tenemos, para el oscilador pesado $(i=0)$, que

$$
P_{0}(t)=D_{0}(t) P_{0}+g(t)
$$

$\operatorname{con} D_{0} \equiv D_{00} \mathrm{y}$

$$
g(t)=\sum_{i} C_{0 i}(t) X_{i}+\sum_{i}^{\prime} D_{0 i} P_{i}
$$

donde $\sum_{i}^{\prime}$ omite al término $i=0$. Si de (3.6) despejamos a $P_{0}$, obtenemos

$$
P_{0}=\frac{P_{0}(t)-g(t)}{D_{0}(t)}
$$

Derivando (3.6) respecto del tiempo y sustituyendo (3.8) obtenemos la ecuación

$$
\dot{P}_{0}(t)=-\gamma(t) P_{0}(t)+f(t)
$$

donde la función $\gamma(t)$ y la "fuerza" $f(t)$ están dadas por

$$
\gamma(t)=-\frac{\dot{D}_{0}(t)}{D_{0}(t)}=-\frac{d}{d t} \ln D_{0}(t)
$$

y

$$
f(t)=\sum_{i}\left[\dot{C}_{i}(t)+\gamma(t) C_{i}(t)\right] X_{i}+\sum_{i}^{\prime}\left[\dot{D}_{i}(t)+\gamma(t) D_{i}(t)\right] P_{i}
$$

respectivamente, siendo $C_{i} \equiv C_{0 i}$ y $D_{i} \equiv D_{0 i}$.

La expresión (3.9) corresponde a una ecuación dinámica exacta. Es de notar que (3.9) tiene la misma forma estructural que la ecuación de Langevin (2.25) si a esta última la escribimos en términos del momento. Sin embargo, las ecuaciones $(2.25)$ y (3.9) tienen diferencias esenciales. En primer lugar la $\gamma$ que aparece en $(2.25)$ es una constante positiva que cuantifica la disipación hidrodinámica, mientras que la $\gamma(t)$ en (3.9) es una función del tiempo que, en general, no corresponde a un coeficiente de fricción. Además, la fuerza $F(t)$ que aparece en la ecuación de Langevin es una fuerza estocástica, mientras que $f(t)$ en la Ec. (3.9) representa una fuerza cuya evolución temporal depende de una manera totalmente determinística de las condiciones iniciales. Lo que tienen en común ambas cantidades es que son fuerzas que tienen su origen en el "fluido". Las observaciones anteriores ponen en claro que tener la solución exacta al problema dinámico no es suficiente; siempre será necesario hacer suposiciones adicionales para poder recuperar la fenomenología del movimiento Browniano. Entonces el problema consiste en mostrar las condiciones bajo las cuales la ecuación determinista (3.9) se reduce a la ecuación de Langevin (2.25), lo que haremos a continuación. 


\subsubsection{Autocorrelación de $P_{0}(t)$}

Nuestra primera observación sobre la solución exacta encontrada anteriormente es que está escrita en términos de las condiciones iniciales del sistema. Sin embargo, en general no hay manera de conocer estas cantidades con precisión. Lo que sí se sabe en las condiciones experimentales usuales es que el "fluido" está inicialmente a determinada temperatura. Esto quiere decir que inicialmente las condiciones iniciales de las partículas del fluido están distribuidas de acuerdo con una distribución canónica. Entonces $\left\{X_{i}(0), P_{i}(0)\right\}$ son cantidades estocásticas que están distribuidas Gaussianamente. Puesto que una de las propiedades de este tipo de variables es que están descorrelacionadas entre sí (esto es, satisfacen $\left\langle X_{i}(0) P_{j}(0)\right\rangle=0$ y $\left.\left\langle P_{i}(0) P_{j}(0)\right\rangle=\delta_{i j}\left(P_{i}^{2}(0)\right\rangle\right)$, podemos entonces multiplicar la solución (3.5) por $P_{0}(0)$ y tomar el promedio sobre una distribución canónica para obtener la función de autocorrelación normalizada del mornento como

$$
D_{0}(t)=\frac{\left\langle P_{0}(t) P_{0}(0)\right\rangle}{\left\langle P_{0}^{2}(0)\right\rangle}
$$

Esta expresión fue obtenida a partir de una propiedad específica de las condiciones iniciales; su forma explícita se encontrará a partir de la solución exacta de las ecuaciones de movimiento.

A continuación desarrollamos las coordenadas y momentos del sistema en términos del conjunto completo de funciones $\left\{U_{i}(k)\right\}$ (ver apéndice $\mathrm{A}$ ) como

$$
\begin{aligned}
X_{i}(t) & =\sum_{k}\left[U_{i}(k) a(k) \cos \omega_{k} t+U_{i}(k) b(k) \sin \omega_{k} t\right] \\
P_{i}(t) & =\sum_{k}\left[U_{i}(k) c(k) \sin \omega_{k} t+U_{i}(k) d(k) \cos \omega_{k} t\right],
\end{aligned}
$$

donde $\omega_{k}$ son las correspondientes eigenfrecuencias, mientras que las amplitudes $a(k), b(k), c(k)$ y $d(k)$ están determinadas por las condiciones iniciales. Si hacemos explícita esta dependencia utilizando la relación de ortonormalidad (A.10) encontramos que

$$
\begin{aligned}
X_{i}(t) & =\sum_{j}\left[X_{j}(0) b_{i j}(t)+P_{j}(0)\left\{\frac{1}{M_{j}} \int_{0}^{t} b_{i j}\left(t^{\prime}\right) d t^{\prime}\right\}\right] \\
P_{i}(t) & =\sum_{j}\left[X_{j}(0)\left\{M_{j} \dot{b}_{i j}(t)\right\}+P_{j}(0) b_{i j}(t)\right],
\end{aligned}
$$

donde

$$
b_{i j}(t)=\sum_{k} M_{j} U_{i}(k) U_{j}^{*}(k) \cos \omega_{k} t
$$


es la solución fundamental del problema, misma que corresponde a las siguientes condiciones iniciales:

$$
P_{i}(0)=\delta_{i 0} \quad \text { y } \quad X_{i}(0)=0 .
$$

Si ahora comparamos la ecuación (3.16) con (3.5) encontramos una relación entre las funciones $C_{i j}$ y $D_{i j}$ de la forma

$$
C_{i j}(t)=M_{i} \dot{D}_{i j}(t),
$$

además de la siguiente expresión para $D_{i j}$ en términos del conjunto completo $\{\mathrm{U}(k)\}$ :

$$
D_{i j}(t)=\sum_{k} M_{j} U_{i}(k) U_{j}^{*}(k) \cos \omega_{k} t
$$

De este último resultado es inmediato tomar la componente deseada, $D_{0}(t) \equiv$ $D_{00}(t)$, la cual se escribe como

$$
D_{0}(t)=M \sum_{\phi_{k}} U_{0}(k) U_{0}^{*}(k) \cos \left(\omega_{0} t \sin \left(\phi_{k} / 2\right)\right),
$$

donde $\phi_{k} \equiv k a=2 \sin ^{-1}\left(\omega_{k} / \omega_{0}\right)$ y

$$
\omega_{0}^{2}=4 \frac{\kappa}{m}
$$

es la frecuencia máxima de oscilación para un cristal perfecto $\left(M_{i}=m \forall i\right)$. Los valores de $\phi_{k}$ corresponden a las frecuencias normales $\omega_{k}$ modificadas por la presencia del defecto, las cuales resultan ser soluciones de la ecuación trascendental (A.21). La amplitud $U_{0}(k)$ se obtiene a partir de la expresión (A.27) tomando $i=0$. Sustituyendo estos resultados en la ec. (3.21) obtenemos

$$
D_{0}(t)=\frac{2 M}{m N} \sum_{\phi_{k}} \frac{\cos \left(\omega_{0} t \sin \left(\phi_{k} / 2\right)\right)}{1+Q^{2} \tan ^{2}\left(\phi_{k} / 2\right)}, \quad 0<\phi_{k}<\pi .
$$

Esta expresión es exacta para $N$ finita y condiciones de frontera periódicas. Aunque estos detalles son irrelevantes cuando se toma el límite termodinámico, es importante tenerlos presentes, ya que este límite sólo se puede tomar rigurosamente de forma analítica. Lo anterior puede hacerse en este modelo de manera exacta, pero en ningun otro más complejo que éste, a menos que se hagan aproximaciones. Y esto es precisamente lo que queremos evitar al usar (3.1) en lugar del Hamiltoniano (2.49) del modelo de fluido ya anteriormente discutido. 
En el límite $N \rightarrow \infty$, la sumatoria sobre la variable discreta $\phi_{k}$ puede sustituirse por una integración sobre la variable continua $\phi$ utilizando la siguiente regla:

$$
\sum_{\phi_{k}} \rightarrow \frac{N}{2 \pi} \int d \phi
$$

por lo que la expresión (3.23) se convierte en

$$
D_{0}(t)=\frac{M}{m} \int_{0}^{\pi} \frac{d \phi}{\pi} \frac{\cos \left(\omega_{0} t \sin (\phi / 2)\right)}{1+Q^{2} \tan ^{2}(\phi / 2)} .
$$

Haciendo el cambio de variable $\omega=\omega_{0} \sin (\phi / 2)$ la expresión anterior se escribe como

$$
\begin{aligned}
D_{0}(t) & =\frac{2}{\pi(Q-1)} \int_{0}^{\omega_{0}} \frac{\left(\omega_{0}^{2}-\omega^{2}\right)^{\frac{1}{2}}}{\omega^{2}+q^{2}} \cos \omega t d \omega \\
& =\frac{1}{\pi(Q-1)} \int_{-\omega_{0} t}^{\omega_{0} t} \frac{\left[\left(\omega_{0} t\right)^{2}-x^{2}\right]^{\frac{1}{2}}}{x^{2}+(q t)^{2}} \cos x d x
\end{aligned}
$$

donde

$$
q=\frac{\omega_{0}}{\left(Q^{2}-1\right)^{1 / 2}} .
$$

Como $\omega_{0}^{-1}$ es el tiempo más pequeño característico de la dinámica del sistema, podemos considerarlo como el análogo de un "tiempo de interacción microscópico". Esto es, para $t \leq \omega_{0}^{-1}$ la interacción entre los osciladores es despreciable y el oscilador pesado, en particular, se comporta como partícula libre. Esperamos entonces que el efecto del baño de osciladores livianos sólo sea apreciable en el comportamiento del oscilador pesado para tiempos tales que $\omega_{0} t \gg 1$. Ahora bien, para obtener una expresión explícita de $D_{0}$ los límites de la segunda igualdad en (3.26) se extienden al infinito y se realiza una integración de contorno en la parte superior del plano complejo que encierra al polo iqt $(>0)$. El resultado es

$$
D_{0}(t)=\frac{Q}{Q-1} \exp \left(-\omega_{0} t /\left(Q^{2}-1\right)^{1 / 2}\right)+I^{\prime},
$$

donde $I^{\prime}$ es el error introducido al extender los límites de integración al infinito.

La forma funcional de $I^{\prime}$ se encuentra integrando la primera igualdad en (3.26) por partes. Despues de algunos arreglos tenemos que 


$$
\begin{aligned}
D_{0}(t)= & \frac{Q+1}{Q^{2}} \frac{J_{1}\left(\omega_{0} t\right)}{\omega_{0} t} \\
& +\frac{1}{\pi(Q-1)}\left\{\left[\left(\omega_{0} t\right)^{2}+(q t)^{2}\right]^{-1} \int_{-\omega_{0} t}^{\omega_{0} t} \frac{x\left[\left(\omega_{0} t\right)^{2}-x^{2}\right]^{\frac{1}{2}}}{x^{2}+(q t)^{2}} \sin x d x\right. \\
& \left.+2 \int_{-\omega_{0} t}^{\omega_{0} t} \frac{x\left[\left(\omega_{0} t\right)^{2}-x^{2}\right]^{\frac{1}{2}}}{\left[x^{2}+(q t)^{2}\right]^{2}} \sin x d x\right\} .
\end{aligned}
$$

Si se extienden los lírnites de integración al infinito y se bacen las integrales de contorno resultantes se encuentra que éstas reproducen el primer término de (3.28). Entonces el término $I^{\prime}$ en (3.28) corresponde al sumando que tiene a la función ordinaria de Bessel de primer orden en esta última expresión. Conservando sólo el primer término de su desarrollo asintótico para $\omega_{0} t \gg 1$ nos da como resultado

$$
\begin{aligned}
D_{0}(t) \rightarrow & \frac{Q}{Q-1} \exp \left(-\omega_{0} t /\left(Q^{2}-1\right)^{1 / 2}\right) \\
& +\frac{Q+1}{Q^{2}}\left(\frac{2}{\pi \omega_{0}^{3} t^{3}}\right)^{\frac{1}{2}} \sin \left(\omega_{0} t-\frac{\pi}{4}\right) .
\end{aligned}
$$

Esta última expresión muestra que la contribución no exponencial tiene caracter oscilatorio con un periodo aproximado de $2 \pi / \omega_{0}$ y amplitud decreciente. Sin embargo, tambien notamos que es de orden $O\left(Q^{-1}\right)$ y entonces, como el caso de interés corresponde a $Q \gg 1$, su contribución a $D_{0}(t)$ puede despreciarse.

Teniendo en cuenta esta última observación podemos sustituir la primera aproximación a $D_{0}(t)$, que es el término exponencial de la Ec. (3.28), en (3.10) para obtener la forma asintótica de la función $\gamma(t)$, cuya forma explícita resulta ser

$$
\gamma(t)=\frac{\omega_{0}}{\left(Q^{2}-1\right)^{1 / 2}} \quad \text { para } \quad t \gg \omega_{0}^{-1} .
$$

Observernos a continuación que, para que este modelo sea físicamente relevante en la descripción del movimiento Browniano, la masa del oscilador central debe de ser mucho mayor que la del resto, por lo que, aplicando esta consideración en la expresión anterior, tenemos como resultado final

$$
\gamma(t) \simeq \Gamma=\left(\frac{m}{M}\right) \omega_{0} \quad \text { cuando } \quad M \gg m .
$$

Puesto que $\Gamma$ resulta ser una constante positiva, hemos recuperado uno de los resultados principales de la fenomenología descrita en el capítulo anterior. Finalmente tenemos que la función de autocorrelación del momento (3.30) toma la 
forma

$$
D_{0}(t) \simeq \exp (-\Gamma t), \quad \text { para } t \gg \omega_{0}^{-1} \text { y } M \gg m
$$

Hagamos ahora algunas observaciones sobre este resultado. Es claro que el límite asintótico en el tiempo y el límite de masa grande tienen que tomarse conjuntamente para obtener la forma exponencial de $D_{0}(t) \mathrm{y}$, por consiguiente, un proceso Markoffiano. Esto es así porque, mientras mayor es la masa del oscilador pesado, mayor tiene que ser el tiempo transcurrido para que sea apreciable el efecto de la interacción con los osciladores del baño. Tenemos entonces que la aproximación $M \gg m$ utilizada para obtener la forma exponencial de $D_{0}(t)$ no es un desarrollo perturbativo; de alguna manera puede considerarse como un granulamiento grueso espacial con el cual se eliminan los detalles dinámicos del modelo, quedándonos con un comportamiento estadístico global. Esto es importante recordarlo, pues sólo la expresión (3.26) describe el comportamiento de $D_{0}(t)$ para cualquier régimen temporal. También es importante mencionar que, si tenemos una masa $M$ grande pero finita, la función $\gamma(t)$ no puede ser estrictamente una constante. Esto nos dice que $M \neq m$ es una condición necesaria, pero no suficiente para obtener movimiento Browniano. Ahora bien, ha podido mostrarse que $m / M \leq 10^{-2}$ es la condición suficiente para que la impureza se comporte como partícula Browniana [45]. Puesto que las contribuciones no exponenciales a $D_{0}(t)$ son de orden $O(m / M)$, éstas se vuelven despreciables si se toma la última condición mencionada. Con esto queremos decir que, si observamos el movimiento de la impureza con un error experimental de un orden mayor a $m / M$, se observará un decaimiento estrictamente exponencial para $D_{0}(t)$. Esto último concuerda muy bien con la fenomenología descrita en el capítulo anterior, puesto que la ecuación de Langevin (2.25) es en si misma el producto de ciertas suposiciones asociadas a los experimentos que establecen su validez.

\subsubsection{Autocorrelación de $f(t)$}

A partir de (3.11) notamos que $f(t)$ depende linealmente de las condiciones iniciales $\left\{X_{i}(0), P_{i}(0)\right\}$ de las partículas de masa $m$ (el "fluido"). Puesto que ya establecimos que las condiciones iniciales son variables aleatorias distribuidas Gaussianamente y $f(t)$ es una combinación lineal de estas variables, entonces $f(t)$ también es una variable aleatoria con una distribución Gaussiana. Esto es así porque la suma de variables aleatorias Gaussianas también es una variable aleatoria con la misma distribución [18]. Falta comprobar si es necesaria alguna otra 
condición para que la función de autocorrelación tenga las propiedades descritas por la teoría fenomenológica del movimiento Browniano.

El cálculo de $\langle f(t) f(0)\rangle$ es inmediato si sustituimos $f(t)$ de (3.9), expresamos a $P(t)$ en términos de las eigenfunciones $U_{i}(k)$ dadas por (A.27) y tomamos el límite $N \rightarrow \infty$. El resultado es [46]

$$
\langle f(t) f(0)\rangle=4 m \int_{0}^{\omega_{0}} \frac{d \omega}{\pi} \cos (\omega(\phi) t)\left(\omega_{0}^{2}-\omega^{2}\right)^{1 / 2} \omega^{2}|b(\phi)|^{2} .
$$

$\mathrm{Si}$ a continuación recordamos que las amplitudes $b(\phi)$ estan determinadas por las condiciones iniciales, entonces podemos hacer la identificación

$$
\omega^{2}|b(\phi)|^{2} \rightarrow \frac{k_{B} T}{2}
$$

misma que permite obtener el resultado

$$
\langle f(t) f(0)\rangle=\Gamma M \omega_{0} \frac{k_{B} T}{2}\left[J_{0}\left(\omega_{0} t\right)+J_{2}\left(\omega_{0} t\right)\right]
$$

donde $J_{n}$ representa a una función ordinaria de Bessel de orden $n$. Para $t \gg \omega_{0}^{-1}$ se tiene un comportamiento de la forma

$$
\frac{1}{2}\left[J_{0}\left(\omega_{0} t\right)+J_{2}\left(\omega_{0} t\right)\right]=\frac{J_{1}\left(\omega_{0} t\right)}{\omega_{0} t} \sim\left(\frac{2}{\pi}\right)^{\frac{1}{2}} \frac{\sin \left(\omega_{0} t-\pi / 4\right)}{\left(\omega_{0} t\right)^{\frac{3}{2}}}
$$

Ahora bien, debido a las oscilaciones presentes en esta última expresión tenemos que la principal contribución a $\int_{-\infty}^{+\infty}\langle f(t) f(0)\rangle d t$ proviene de la región $\left(-\omega_{0}^{-1},+\omega_{0}^{-1}\right)$. Si deseamos que (3.37) sea una representación adecuada de una función $\delta$ con un error de aproximadamente $1 \%$, entonces la unidad de tiempo más pequeña que debe tomarse es de $20 / \omega_{0}$ [45]. Este paso corresponde a realizar un granulamiento grueso temporal cuyo efecto es el de eliminar los detalles inherentes al modelo, como en su contraparte espacial. Tenemos finalmente que

$$
\langle f(t) f(0)\rangle=2 \Gamma M k_{B} T \delta(t) \quad t \sim 20 / \omega_{0}
$$

la cual es la expresión postulada por la teoría fenomenológica del capítulo precedente. 


\subsubsection{Ecuación de Langevin}

Teniendo en cuenta los resultados anteriores tenemos que (3.9) se puede reducir a la ecuación fenomenológica de Langevin ${ }^{1}$

$$
\dot{P}(t)=-\Gamma P(t)+f(t)
$$

donde $P(t) \equiv P_{0}(t)$ y $\Gamma \equiv \gamma / M$ ( $\gamma$ es el coeficiente de fricción definido en el capítulo anterior). Además $f(t)$ es una fuerza estocástica que cumple con la propiedad

$$
\langle f(t) f(0)\rangle=2 \Gamma M k_{B} T \delta(t),
$$

mientras que la función de autocorrelación $D_{0}(t)$ tiene la forma exponencial dada por

$$
\langle P(t) P(0)\rangle=M k_{B} T \exp (-\Gamma t),
$$

donde hemos hecho uso del teorema de equipartición $\left\langle P^{2}(0)\right\rangle=M k_{B} T$.

Sin embargo, la validez de estos resultados no es general (para cualquier tiempo), sino que, para éste modelo en particular, está restringida al intervalo

$$
\omega_{0}^{-1}<t<\Gamma^{-1} \ln \left(\frac{M}{m}\right)
$$

La existencia de este régimen temporal fue mencionada por Hemmer [47] y posteriormente por Takeno y Hori [48], pero los límites arriba mencionados fueron calculados de manera explícita por Ullersma [49]. El hecho de que el proceso sea Markoffiano sólo en este intervalo es una consecuencia de la forma particular del potencial a primeros vecinos elegido para escribir el Hamiltoniano (3.1), el cual establece que existe una frecuencia máxima de oscilación $\omega_{0}$. La existencia de este acotamiento en las posibles frecuencias del sistema se traduce en el ya mencionado intervalo de validez. Es claro entonces que (3.42) impone una restricción importante al modelo de osciladores armónicos como fundamentación microscópica del movimiento Browniano. Ahora bien, en principio esta limitación no es tan restrictiva como parece a primera vista ya que, como mencionamos antes, las contribuciones no exponenciales a $D_{0}(t)$ se vuelven despreciables cuando $M \gg m$. Para

\footnotetext{
${ }^{1}$ Cabe mencionar que, para el modelo definido por (3.1), se puede construir una FDP conjunta para la posición y el momento del oscilador pesado. A partir de esta última se pueden definir las funciones de densidad de probabilidad para el momento y la posición del oscilador pesado, mismas que satisfacen unas ecuaciones tipo Fokker-Planck y de difusión, respectivamente [49]
} 


\subsubsection{Ecuación de Langevin}

Teniendo en cuenta los resultados anteriores tenemos que (3.9) se puede reducir a la ecuación fenomenológica de Langevin ${ }^{1}$

$$
\dot{P}(t)=-\Gamma P(t)+f(t),
$$

donde $P(t) \equiv P_{0}(t)$ y $\Gamma \equiv \gamma / M$ ( $\gamma$ es el coeficiente de fricción definido en el capítulo anterior). Además $f(t)$ es una fuerza estocástica que cumple con la propiedad

$$
\langle f(t) f(0)\rangle=2 \Gamma M k_{\theta} T \delta(t),
$$

mientras que la función de autocorrelación $D_{0}(t)$ tiene la forma exponencial dada por

$$
\langle P(t) P(0)\rangle=M k_{H} T \exp (-\Gamma t),
$$

donde hemos hecho uso del teorema de equipartición $\left\langle P^{2}(0)\right\rangle=M k_{B} T$.

Sin embargo, la validez de estos resultados no es general (para cualquier tiempo), sino que, para éste modelo en particular, está restringida al intervalo

$$
\omega_{0}^{-1}<t<\Gamma^{-1} \ln \left(\frac{M}{m}\right) .
$$

La existencia de este régimen temporal fue mencionada por Hemmer [47] y posteriormente por Takeno y Hori [48], pero los límites arriba mencionados fueron calculados de manera explícita por Ullersma [49]. El hecho de que el proceso sea Markoffiano sólo en este intervalo es una consecuencia de la forma particular del potencial a primeros vecinos elegido para escribir el Hamiltoniano (3.1), el cual establece que existe una frecuencia máxima de oscilación $\omega_{0}$. La existencia de este acotamiento en las posibles frecuencias del sistema se traduce en el ya mencionado intervalo de validez. Es claro entonces que (3.42) impone una restricción importante al modelo de osciladores armónicos como fundamentación microscópica del movimiento Browniano. Ahora bien, en principio esta limitación no es tan restrictiva como parece a primera vista ya que, como mencionamos antes, las contribuciones no exponenciales a $D_{0}(t)$ se vuelven despreciables cuando $M \gg m$. Para

\footnotetext{
${ }^{1}$ Cabe mencionar que, para el modelo definido por (3.1), se puede construir una FDP conjunta para la posición y el momento del oscilador pesado. A partir de esta última se pueden definir las funciones de densidad de probabilidad para el momento y la posición del oscilador pesado, mismas que satisfacen unas ecuaciones tipo Fokker-Planck y de difusión, respectivamente [49]
} 
el caso de un sistema finito esta limitante tampoco provoca problemas de consideración, pues las escalas de tiempo que aparecen en (3.42) son muy inferiores al tiempo de recurrencia del sistema [47]. Sin embargo, como la existencia de (3.42) depende de los detalles del modelo, han habido intentos de superar esta limitante dentro del contexto de los modelos de osciladores armónicos, mismos que serán el tema de nuestra siguiente sección.

\subsection{Interacciones de Largo Alcance}

Todos los autores ya citados han trabajado con el Hamiltoniano (3.1), el cual define a un sistema de osciladores con interacciones a primeros vecinos, como en un sólido unidimensional. Sin embargo, desde un punto de vista teórico es posible construir modelos unidimensionales en los que un oscilador dado esté acoplado armónicamente no solo a sus primeros vecinos, sino también a otros más alejados. Esta es una forma de ampliar el rango de aplicabilidad de los modelos de osciladores, con la ventaja adicional de que la dinámica sigue siendo resoluble de manera exacta. Por lo tanto, el estudio del comportamiento estadístico del sistema puede hacerse analíticamente, como en la sección anterior. Siguiendo esta metodología puede confirmarse hasta qué punto la ecuación de Langevin, y todos los resultados que esta implica, es independiente de los detalles de la interacción, al menos para los sistemas de osciladores armónicos.

El primer y más célebre trabajo en el que se exploró que tipo de interacción puede reproducir la forma exponencial de la autocorrelación del momento fue el de Ford, Kac y Mazur [50]. En este trabajo se planteó un modelo que está descrito por el Hamiltoniano

$$
H=\frac{1}{2}\left[\sum_{i=-N / 2}^{N / 2} P_{i}^{2}+\sum_{i, j=-N / 2}^{N / 2} X_{i} \Phi_{i j} X_{j}\right] .
$$

En esta expresión $X_{i}$ y $P_{i}$ son las coordenadas y momentos del $i$-ésimo oscilador, respectivamente, mientras que $\Phi_{i j}$ es el elemento de la matriz simétrica $\Phi$ que cuantifica la interacción entre el $i$-ésimo y el $j$-ésimo oscilador de la cadena. Obsérvese que, a diferencia del modelo con interacciones a primeros vecinos que ya hemos estudiado, en el presente modelo la masa de todos los osciladores, en particular la del central, es la misma.

Las soluciones formales a las ecuaciones de movimiento que se obtienen a partir 
del Hamiltoniano (3.43) se escriben como

$$
\begin{aligned}
X_{i}(t) & =\sum_{j}\left[\left(\cos \Phi^{\frac{1}{2}} t\right)_{i j} X_{j}(0)+\left[\Phi^{-\frac{1}{2}}\left(\sin \Phi^{\frac{1}{2}} t\right)\right]_{i j} P_{j}(0)\right] \\
P_{i}(t) & =\sum_{j}\left[-\left[\Phi^{\frac{1}{2}}\left(\sin \Phi^{\frac{1}{2}} t\right)\right]_{i j} X_{j}(0)+\left(\cos \Phi^{\frac{1}{2}} t\right)_{i j} P_{j}(0)\right]
\end{aligned}
$$

donde las funciones trigonométricas que aparecen se definen por sus desarrollos en serie. A continuación supondremos, por las mismas razones expuestas en el caso de primeros vecinos, que el sistema está en equilibrio termodinámico en el instante inicial. Esto implica que las condiciones iniciales $\left\{X_{i}(0), P_{i}(0)\right\}$ son variables aleatorias obtenidas a partir de una distribución Gaussiana, como ya habíamos antes mencionado. Debido a la naturaleza lineal de las soluciones $\left\{X_{i}(t), P_{i}(t)\right\}$, tenemos entonces un proceso Gaussiano estacionario cuyas propiedades estadísticas están determinadas por las funciones de correlación $\left\langle X_{i}(0) X_{j}(t)\right\rangle,\left\langle X_{j}(0) P_{j}(t)\right\rangle$ y $\left\langle P_{i}(0) P_{j}(t)\right\rangle$. En particular, a partir esta última se puede expresar la función de autocorrelación normalizada del momento para el oscilador central de la cadena como

$$
\rho_{0}(t) \equiv \frac{\left\langle P_{0}(t) P_{0}(0)\right\rangle}{\left\langle P_{0}^{2}(0)\right\rangle}=\left(\cos \Phi^{\frac{1}{2}} t\right)_{00} .
$$

Entonces el problema planteado por Ford et al. es el de determinar qué propiedades deberían de tener las constantes de acoplamiento del cristal (dadas por $\boldsymbol{\Phi}$ ) para que la función de autocorrelación normalizada del momento tenga la forma

$$
\rho_{0}(t)=\exp \left(-\Gamma_{F}|t|\right)
$$

lo que garantiza que el proceso sea Markoffiano.

Hagamos notar, en primer lugar, que $\left(\cos \boldsymbol{\Phi}^{\frac{1}{2}} t\right)_{00}$ es una función de la matriz de acoplamiento $\boldsymbol{\Phi}$; esto es, $\left(\cos \boldsymbol{\Phi}^{\frac{1}{2}} t\right)_{00} \equiv[F(\boldsymbol{\Phi})]_{00}$. Ahora bien, esta función se puede expresar como la tranformada de Fourier de una cierta función $f(\theta)$ de la forma

$$
[F(\Phi)]_{i j}=\frac{1}{2 \pi} \int_{-\pi}^{\pi} F(f(\theta)) \cos ((i-j) \theta) d \theta,
$$

donde $f(\theta)=\omega^{2}$. Entonces el requerimiento de que $\rho_{0}(t)$ sea una función exponencial decreciente puede escribirse como

$$
\rho_{0}(t)=\frac{1}{2 \pi} \int_{-\pi}^{\pi} \cos \left([f(\theta)]^{\frac{1}{2}} t\right) d \theta=\exp \left(-\Gamma_{F}|t|\right) .
$$


Esta es una ecuación integral para $f(\theta)$ cuya solución es

$$
f(\theta)=\Gamma_{F}^{2} \tan ^{2} \frac{1}{2} \theta
$$

Aqui se presenta una dificultad importante, pues los elementos de matriz $\boldsymbol{\Phi}$ a los que conduce (3.50) divergen debido a la singularidad de la solución en $\theta=\pi$. Para resolver este problema Ford et al. introdujeron una frecuencia de corte $\theta_{0}$ tal que

$$
f^{*}(\theta)=\left\{\begin{array}{lll}
\Gamma_{F}^{2} \tan ^{2} \frac{1}{2} \theta, & \text { si } & |\theta|<\theta_{0} \\
0 & \text { si } & \theta_{0} \leq|\theta| \leq \pi
\end{array}\right.
$$

Esta suposición soluciona formalmente el problema de determinar las condiciones suficientes para que la autocorrelación del momento corresponda a un proceso Markoffiano, aunque con la salvedad de que la segunda igualdad en (3.49) es válida sólo para el limite $\omega_{0}=\Gamma_{k} \tan \frac{1}{2} \theta_{0} \rightarrow \infty$. Esto es claro si sustituimos $f^{*}(\theta)$ en (3.49) y cambiarnos la variable de integración a $\omega=\Gamma_{F} \tan \frac{1}{2} \theta$, con lo que resulta la representación

$$
\rho_{0}(t)=\frac{1}{\pi} \int_{-\omega_{0}}^{\omega_{0}} \frac{\Gamma_{k}}{\omega^{2}+\Gamma_{r^{2}}^{2}} \cos (\omega t) d \omega
$$

la cual tiende a $\exp \left(-\Gamma_{F}|t|\right)$ cuando $\omega_{0} \rightarrow \infty$.

Una objeción importante al trabajo de Ford et al. es que, tanto la frecuencia de corte $\omega_{0}$ como el límite $\omega_{0} \rightarrow \infty$ son condiciones necesarias para obtener movimiento Browniano, pero cuya única justificación es la validez de los resultados obtenidos a través de ellas; además, no resulta nada clara la interpretación física de la interacción dada por (3.50). Por esto es que dificilmente puede considerársele como la solución completa al problema planteado por las interacciones de corto alcance, aunque indudablemente significó un gran avance en el estudio del movimiento Browniano a través de los modelos de osciladores armónicos.

Posteriormente, Phillipson [51] trabajó este mismo problema (esto es, con osciladores de masa idéntica) pero utilizando un modelo explícito de interacciones de largo alcance, en el cual el Hamiltoniano (3.43) toma la siguiente forma:

$$
H=\frac{1}{2}\left[\sum_{i=1}^{N+1} P_{i}^{2}+\sum_{j=1}^{L \leq N / 2} \sum_{i=1}^{(N+1)-j} \kappa_{j}\left(X_{i+j}-X_{i}\right)^{2}\right]
$$

$L=1$ corresponde al caso de interacciones a primeros vecinos, mientras que $L=N / 2$ corresponde a una interacción de máximo alcance entre osciladores. Las 
constantes de fuerza $\kappa_{j}$ cuantifican el grado de acoplamiento entre el oscilador $i$ y otro oscilador a una distancia $j d$ del primero. Las posibles elecciones de las constantes de fuerza están dictadas por dos criterios. El primero es que las eigenfrecuencias resultantes sean reales, y el segundo es que la fuerza de interacción entre osciladores disminuya conforme aumente la distancia entre ellos,

$$
\left|\kappa_{j}\right|>\left|\kappa_{j+1}\right| ; \quad \lim _{L, N \rightarrow \infty}\left|\kappa_{L}\right|=0 .
$$

A continuación se plantea un modelo explícito para las constantes de fuerza en el cual estas están relacionadas entre sí como los términos de una progresión geométrica con signo alternado de la forma

$$
\kappa_{j}=(-z)^{j-1} \kappa ; \quad j=1,2, \ldots, \infty, \quad|z|<1 .
$$

En este modelo la variable $z$ parametriza el alcance de las interacciones entre osciladores. A partir de (3.53) junto con (3.55) se puede calcular la expresión de la densidad de frecuencias $g(\omega)$ (número de frecuencias por unidad de intervalo de frecuencia entre el número total de frecuencias) para este modelo, la cual admite cualquiera de las siguientes expresiones explícitas

$$
\begin{aligned}
g(\omega) & =\frac{2}{\pi} \frac{\left(\omega_{0}^{P}\right)^{2}\left(1-z^{2}\right)}{\left[\left(\omega_{0}^{P}\right)^{2}(1-z)^{2}+4 z\left(\omega_{0}^{P}\right)^{2}\right]\left(\left[\omega_{0}^{P}\right]^{2}-\omega^{2}\right)^{1 / 2}} \\
& =\frac{(1+z)}{\pi z^{1 / 2}} \frac{\omega_{0}^{P} \Gamma_{P}}{\left(\Gamma_{p}^{2}+\omega^{2}\right)\left(\left[\omega_{0}^{P}\right]^{2}-\omega^{2}\right)^{1 / 2}}
\end{aligned}
$$

donde las cantidades

$$
\omega_{0}^{P}=\left[\frac{4 \kappa}{m\left(1-z^{2}\right)}\right]^{1 / 2} \quad \text { y } \quad \Gamma_{p}=\frac{1-z}{2\left(z^{1 / 2}\right)} \omega_{0}^{P}
$$

actúan recíprocamente como funciones de $z$. El primer caso límite de interés se obtiene tomando $z=0$, por lo que $\omega_{0}^{P} \equiv \omega_{0}=2(\kappa / m)^{1 / 2}, \Gamma_{P} \rightarrow \infty$ y la expresión para $g(\omega)$, de (3.56), resulta

$$
g(\omega)=\frac{2}{\pi\left(\omega_{0}^{2}-\omega^{2}\right)^{1 / 2}}
$$

siendo ésta la expresión explícita para la densidad de frecuencias en el caso de interacciones a primeros vecinos ya estudiado con anterioridad. Ahora, cuando $z \rightarrow 1$, tenemos que $\omega_{0}^{P} \rightarrow \infty, \Gamma_{P} \rightarrow 0$ y entonces tenemos, a partir de (3.57),

$$
g(\omega) \rightarrow \frac{2 \Gamma_{p}}{\pi\left(\Gamma_{P}^{2}+\omega^{2}\right)},
$$


que corresponde a la densidad de frecuencias calculada por Ford et al. [50] por medio de

$$
g(\omega)=\frac{2 \omega}{\pi f^{\prime}(\theta)},
$$

donde $\theta$ es la función de $\omega$ obtenida por inversión de la ecuación $\omega^{2}=f(\theta)$ (por lo que $g(\omega)$ es el jacobiano de la transformación) y la función $f(\theta)$ está dada por la expresión (3.50), la cual permite obtener la forma exponencial para la función de autocorrelación del momento.

Ahora bien, a partir de la densidad de frecuencias $g(\omega)$ correspondiente al modelo de interacción definido por (3.55) se puede calcular las expresión general para la función de autocorrelación del momento a partir de

$$
\rho_{0}(t)=\int_{0}^{\omega_{0}}(g(\omega) \cos \omega t) d \omega,
$$

resultando una expresión que, para $0.8 \leq z<1$ (interacciones de muy largo alcance) y $\omega_{0} t \geq 10$ (comportamiento asintótico), se puede escribir como

$$
\rho_{0}(t)=\exp \left(-\Gamma_{p} t\right)+\mu_{p}\left(\frac{2}{\pi \omega_{0} t}\right)^{\frac{1}{2}} \cos \left(\omega_{0} t-\frac{\pi}{4}\right)
$$

donde $\mu_{p}$ es un parámetro que esta relacionado con $z$ por medio de $z=1-2 \mu_{p}$ y cuya dependencia explícita con respecto a $\omega_{0}^{P}$ y $\Gamma_{p}$, en el límite $z \rightarrow \infty$, se muestra en el apéndice B. Si este resultado se compara con (3.30) podemos hacer varias observaciones interesantes. La primera es que la parte oscilatoria de este modelo se comporta corno $\omega_{0} t^{-1 / 2}$, mientras que, para el caso del modelo de vecinos próximos, se tiene un comportamiento de la forma $\omega_{0} t^{-3 / 2}$ (ver Ec. (3.30)). La segunda es que, despreciando estos términos oscilatorios, ambos modelos predicen asintóticamente un decaimiento exponencial. De hecho, como se muestra en el apéndice $\mathrm{B}$, si en el modelo de vecinos próximos hacemos la transformación $M \rightarrow m$ y $m \rightarrow m \mu_{p} / 4$, los factores exponenciales en ambos modelos, Ecs. (3.30) y (3.63), son formalmente idénticos. Esto sugiere que, aunque en este modelo todos los osciladores tengan idéntica masa, la función de autocorrelación del momento corresponde a la de un oscilador "pesado" de masa $m$ inmerso en una cadena de osciladores "livianos" de masa $m \mu_{p} / 4$ y con acoplamiento a primeros vecinos entre todos ellos. Este resultado muestra que, para el caso de osciladores armónicos de masas idénticas, cualquiera de ellos puede realizar movimiento Browniano si las interacciones entre osciladores son de muy largo alcance. Queda aclarada, además, la contribución del trabajo de Ford et al., ya que en éste no era del todo evidente 
que (3.50) implicara interacciones de muy largo alcance, como es el caso con este modelo.

Aunque el análisis realizado en los dos trabajos anteriores es sumamente elegante, un modelo de osciladores con masas iguales no es realmente un buen análogo al fenómeno físico del movimiento Browniano. El modelo trabajado originalmente por Rubin [44] y definido por (3.1) está mucho más cerca de la situación física en la que una partícula pesada está acoplada a un conjunto de partículas mucho más livianas que ella. En el caso específico de interacciones a primeros vecinos ya hemos visto que la consecuencia más importante de este tipo de acoplamiento es la existencia del intervalo de validez (3.42). Por otra parte, para los sistemas de osciladores de masa idéntica hemos visto que cualquiera de ellos puede efectuar movimiento Browniano con la condición de que las interacciones sean de muy largo alcance. Sin embargo, esta es una condición poco plausible físicamente, además de que la validez de los resultados obtenidos depende de la forma particular (3.55) de las constantes de acoplamiento entre los osciladores. Teniendo en cuenta estos antecedentes podría entonces tratarse de encontrar cuáles son las condiciones físicas más generales bajo las cuales un defecto pesado realiza movimiento Browniano independientemente del alcance de la interacción entre los osciladores. Siguiendo esta linea de ideas Mazur y Braun [52] encontraron que la impureza realiza movimiento Browniano en el límite $M \rightarrow \infty$, $t \rightarrow \infty$ de tal forma que $t / M$ permanezca finito, el cual no es otro que el límite de acoplamiento débil ya mencionado en el capítulo anterior ${ }^{2}$. Este límite define una nueva escala de tiempo $\tau=t / M$ en la cual es rigurosamente válida la ecuación fenomenológica de Langevin (3.39), así como las propiedades estocásticas de la fuerza fluctuante (3.40) y el decaimiento exponencial de la autocorrelación del momento (3.41). La única condición necesaria que hay que imponer sobre el alcance de la interacción (cuantificada a través de la densidad de frecuencias $g(\omega)$ ) es que $g(0)$ sea una cantidad finita no nula. De (3.60) y (3.59) vemos que esta condición se satisface trivialmente tanto para interacciones de muy largo como de corto alcance. Por lo tanto podemos decir que ha sido demostrado explícitamente que las condiciones implicadas por el límite del acoplamiento débil garantizan que el oscilador pesado realice movimiento Browniano independientemente de los detalles de la interacción, al menos para los modelos de osciladores armónicos. Sin embargo, y a pesar de su innegables logros, este enfoque tiene limitaciones im-

\footnotetext{
${ }^{2}$ Un análisis detallado de la aplicación de este límite al modelo de osciladores acoplados siguiendo la metodología de [52] se puede estudiar en el apéndice C
} 
portantes. La principal es que los resultados obtenidos sólo son válidos cuando se tiene rigurosamente que $M=\infty$. Para un valor finito de $M$ (no importando qué tan grande sea) la función de autocorrelación del mornento $\rho_{0}(t)$ muestra un comportamiento oscilatorio para tiempos grandes [45], como ya antes mencionamos. Esto último es consecuencia de que el término de decaimiento exponencial en $\rho_{0}(t)$ nunca aparece aisladamente mientras $\omega_{0}$ tenga un valor finito [48]. Por último, en la escala natural de tiempo $t$ es imposible observar movimiento alguno en el ascilador pesado.

\subsection{Conclusiones}

El objetivo del presente capítulo ha sido el de mostrar cómo se puede deducir la mayor parte de la fenomenología del movimiento Browniano a partir de modelos microscópicos sencillos. En particular, hemos mostrado cómo estos modelos simplifican enormemente el problema de calcular los coeficientes de trasporte, tarea que resultaba extremadamente complicada en los modelos de fluido ya anteriormente reseñados. Ahora bien, retomando la discusión iniciada al principio del capítulo, y teniendo en cuenta lo hasta ahora presentado, volvemos a preguntarnos cuáles de las resultados obtenidos son inherentes a los modelos de osciladores y cuáles de ellos son resultados generales del movimiento Browniano aplicables a sisternas mucho más complicados, tales como fluidos. Una observación favorable en este sentido es que (3.62) implica que $g(\omega)$ es la transformada de Fourier de la función de autocorrelación del momento. Esta relación es válida en líquidos, siendo $g(\omega)$ conocida como densidad espectral. Otra observación favorable es que el comportamiento asintótico de la autocorrelación del momento $\left(\sim t^{-3 / 2}\right)$ ha sido observado en estudios de fluidos por medio de dinámica molecular (aunque su observación experimental todavía es motivo de controversia, como ya se mencionaba en el capítulo 2). De hecho, la presencia de este comportamiento asintótico es perfectamente congruente con los resutados generales de Lee [53], el cual demostró que no es posible obtener un decaimiento exclusivamente exponencial para $\rho_{0}(t)$ partiendo de consideraciones puramente dinámicas. Por el lado negativo podemos decir que estos modelos, por sus misma naturaleza, son incapaces de modelar fenómenos inherentes a los fluidos, tales como las interacciones hidrodinámicas. Sin embargo, en el contexto de la teoría del movimiento Browniano, la limitación más importante que tienen este tipo de modelos es que las interacciones armónicas en los que están basados no son particularmente realistas para modelar el tipo de 
interacción que existe en fluidos reales. Para el estudio de éstos, los potenciales más comunmente utilizados (Morse, Lennard-Jones, etc.) tienen un caracter marcadamente diferente al potencial armónico que hemos estudiado hasta este momento. Desde esta perspectiva sería entonces deseable explorar la posibilidad de construir un modelo anarmónico que ampliara el rango de aplicabilidad de los modelos armónicos, pero que todavía mantuviera muchas de sus ventajas. Para conseguir esto último el punto de partida natural sería el Hamiltoniano (3.1), al cual podría añadirse un potencial anarmónico convenientemente elegido. Ahora bien, para el estudio de ciertos fenómenos de interés en la mecánica estadística (entre los cuales no ha estado incluido hasta ahora el movimiento Browniano), ya han sido planteados y estudiados exhaustivamente modelos basados en la propuesta antes mencionada, por lo que haremos una revisión de los mismos en el capítulo siguiente. 


\section{Capítulo 4}

\section{El Modelo Fermi-Pasta-Ulam}

\subsection{Introducción}

Como hemos podido ver en el capítulo anterior, los modelos de osciladores armónicos permiten el estudio analítico exacto de algunos efectos de la dinámica de muchos cuerpos. Aunque la discusión se concentró en su viabilidad como modelo microscópico para el estudio del movimiento Browniano, su utilidad ha sido más ampliamente comprobada en el estudio de las propiedades termodinámicas de sólidos cristalinos (especialmente del calor específico) y en la relación entre las propiedades elásticas macroscópicas y las constantes de fuerza microscópicas. Se ha estudiado además la relación entre las propiedades dinámicas (funciones de autocorrelación de posición y momento) de átomos individuales y ciertas propiedades experimentales para los casos de átomos individuales en una red cristalina perfecta, de átomos en la vecindad de una superficie y de defectos livianos y pesados [54].

Pero incluso en el estudio de sólidos, los modelos de osciladores armónicos no son los suficientemente generales como para estudiar todos los fenómenos de interés. Por ejemplo, para el problema de la conductividad térmica es perfectamente conocido que estos sistemas son incapaces de modelar correctamente la fenomenología macroscópica, como ya habíamos mencionado en la introducción del capítulo 3. De hecho, para este problema se consideró desde hace mucho tiempo la posibilidad de ampliar el intervalo de validez de los modelos de osciladores armónicos del tipo descrito por el Hamiltoniano (3.1), pero incluyendo términos potenciales de más alto grado (esto es, anarmónicos). Sin embargo, un estudio analítico exacto del problema se vuelve imposible, por lo que se han usado distintos tipos de aproximaciones, ya sea analíticas (teoría de perturbaciones, princi- 
palmente) o numéricas. Este último enfoque fue iniciado por el célebre trabajo de E. Fermi, J. Pasta y S. Ulam de 1954 , el cual fue quizas el primer "experimento numérico" realizado en la historia y que inició toda una meva era tanto para la mecánica estadística como para el estudio de los sistemas dinámicos, además de iniciar una metodología que es conocida actualmente como dinámica molecular. Una excelente reseña histórica de estos desarrollos puede consultarse en el artículo de revisión sobre el tema de J. Ford [55].

En la primera sección presentaremos brevemente en qué consistió el trabajo original de Fermi et al., para continuar con una revisión de los trabajos posteriores que se han relizado para interpreiar y ampliar los resultados obtenidos por estos autores. A continuación revisaremos el enfoque que se la ha dado al problema desde el punto de vista de los sistemas dinámicos, haciendo un recuento de los teoremas hasta ahora conocidos para estudiar la evolución temporal de los sistemas Hamiltonianos no-armónicos y su relevancia para el caso de un número infinito de grados de libertad. De especial importancia será reseñar los distintos parámetros que han sido propuestos para detectar numéricamente la presencia de trayectorias no-regulares en el espacio fase de estos sistemas y estudiar la estructura del mismo, así como su aplicación específica al modelo de Fermi et al.

\subsection{El Problema de la Equipartición}

De acuerdo al punto de vista tradicional, un sistema dado, no importando cómo sea preparado, evoluciona en una escala de tiempo razonable a un estado de equilibrio termodinámico. Una de las características más importantes de este estado de equilibrio es que la energía del sistema está repartida por igual entre todos los grados de libertad que aparecen cuadráticamente en el Hamiltoniano. Este enunciado corresponde al así llamado principio de equipartición, el cual ya hemos utilizado en el contexto del movimiento Browniano. Ahora bien, siendo este uno de los resultado más fundamentales de la mecánica estadística clásica, su violación en cierta situación particular tendría consecuencias importantes. Baste recordar que, a finales del siglo XIX, la confianza en el principio de equipartición era $\tan$ grande entre lo físicos de aquel entonces que la falta de equipartición observada en la radiación de cuerpo negro fue considerada como evidencia del fracaso de la mecánica clásica para explicar fenómenos a nivel microscópico ${ }^{1}$.

\footnotetext{
${ }^{1}$ Para una reseña de la relación histórica entre las propiedades de la radiación del cuerpo negro y el problema de la equipartición en el modelo FPU ver Ref. [56]
} 


\subsubsection{El Trabajo Original}

La idea original del reporte de Fermi et al. [57] fue la de comprobar numéricamente si un sistema dinámico no-lineal de muchos cuerpos pudiera comportarse, para tiempos muy grandes, como un sistema mecanico-estadístico; esto es, que relajase hacia el estado de equilibrio. Para conseguir este objetivo, decidieron integrar numéricamente las ecuaciones de movimiento correspondientes a un sistema unidimensional de $N$ partículas de masa unitaria interaccionando a primeros vecinos a través de una constante de acoplamiento armónica también unitaria, extremos fijos $\left(X_{0}=X_{N+1}=0\right)$ y descrito por el Hamiltoniano

$$
H=\sum_{i=0}^{N}\left[\frac{1}{2} \dot{X}_{i}^{2}+\frac{1}{2}\left(X_{i+1}-X_{i}\right)^{2}+\frac{\alpha}{3}\left(X_{i+1}-X_{i}\right)^{3}\right],
$$

el cual define al actualmente conocido modelo FPU- $\alpha$. Físicamente su estructura es muy razonable, ya que (4.1) puede considerarse como la primera aproximación anarmónica a potenciales de interacción más realistas, tales como el de Morse o de Lennard-Jones.

Una forma equivalente de escribir al Hamiltoniano de este sistema se consigue al efectuar la siguiente transformación canónica ortogonal

$$
Q_{i}(t)=\left(\frac{2}{N}\right)^{\frac{1}{2}} \sum_{n=1}^{N} \sin \left(\frac{\pi n i}{N}\right) X_{n}(t)
$$

que diagonaliza a la parte armónica de (4.1), lo que resulta en la siguiente representación del Hamiltoniano

$$
H=\sum_{i=1}^{N}\left[\frac{1}{2} \dot{Q}_{i}^{2}+\frac{1}{2} \omega_{i}^{2} Q_{i}^{2}+\alpha \sum_{j, k=1}^{N} C(i, j, k) Q_{i} Q_{j} Q_{k}\right],
$$

donde $\omega_{i}=2 \sin \left(\frac{i \pi}{2 N}\right)$. Vemos que, en términos de las variables $Q_{i}$, conocidas como coordenadas normales, se tiene un conjunto de $N$ modos de vibración acoplados por medio de la constante $\alpha$. Tales modos de vibración se conocen como modos normales y es claro que, si $\alpha=0$, se tiene un conjunto de $N$ osciladores armónicos independientes, donde $\omega_{i}$ es la frecuencia del $i$-ésimo modo normal y $E_{i}=\frac{1}{2}\left(\dot{Q}_{i}(t)+\omega_{i}^{2} Q_{i}^{2}(t)\right)$ es su correspondiente energía. Para el caso $\alpha \neq 0$ esta última es sólo una expresión aproximada, pero que todavía puede considerarse válida si $\alpha \ll 1$. Es en este contexto que la relajación hacia el equilibrio corresponde a obtener la equipartición de la energía entre todos los grados de 
libertad (modos normales) del sistema. Es claro entonces que el término cúbico es el responsable del intercambio de energía entre los modos normales.

Las condiciones iniciales del experimento numérico original consistieron en excitar a un solo modo normal. Esto es, el desplazamiento inicial de las partículas de sus posiciones de equilibrio está dado por el modo fundamental

$$
X_{i}(0)=A \sin \left(\frac{\pi n i}{N}\right), \quad i=1, \ldots, N,
$$

donde $n=1$, mientras que las velocidades iniciales son $\dot{X}_{i}(0)=0 \forall i$. Fermi et al. utilizaron $N=32, A=1$ y $\alpha=0.25$, siendo la energía total $E=0.077$, o bien $\epsilon=0.00241$, donde $\epsilon=E / N$ es la densidad de energía. Estas condiciones implican que toda la energía del sistema está concentrada en el modo fundamental, y ellos esperaban observar, en un tiempo de cómputo razonable, un flujo significativo de energía del modo fundamental a los otros modos normales. Sin embargo, el experimento tuvo un resultado totalmente inesperado, como puede apreciarse en la figura 4.1. En palabras de los autores:

Digamos que los resultados de nuestros cálculos, desde el principio, nos sorprendieron. En luggar de un fiujo continuo de énergía del primer modo a los modos más altos, todos los casos mostraron comportamientos completamente diferentes. ... En lugar de un incremento gradual de todos los modos más altos, la energía se intercambia, esencialmente, sólo entre algunos pocos. Por lo tanto, es muy dificil observar el ritmo de "termalización" o mezclado en nuestro problema, y este era el propósito inicial de nuestros cólculos'.

Otro modelo estudiado por Fermi et al tiene un potencial cuártico con un Hamiltoniano de la forma

$$
H=\sum_{i=0}^{N}\left[\frac{1}{2} \dot{X}_{i}^{2}+\frac{1}{2}\left(X_{i+1}-X_{i}\right)^{2}+\frac{\beta}{4}\left(X_{i+1}-X_{i}\right)^{4}\right],
$$

el cual también proporciona resultados similares a los obtenidos con (4.1). Este Hamiltoniano ha sido conocido en la literatura como modelo FPU- $\beta$ y será de una importancia crucial en el desarrollo del presente trabajo.

\footnotetext{
${ }^{2}$ A pesar de esta última afirmación, muchos años después uno de los autores del reporte original (J. Pasta) mencionó que el objetivo original del trabajo de 1954 era estudiar la conductividad térmica del sistema. Ver Ref. [58]
} 


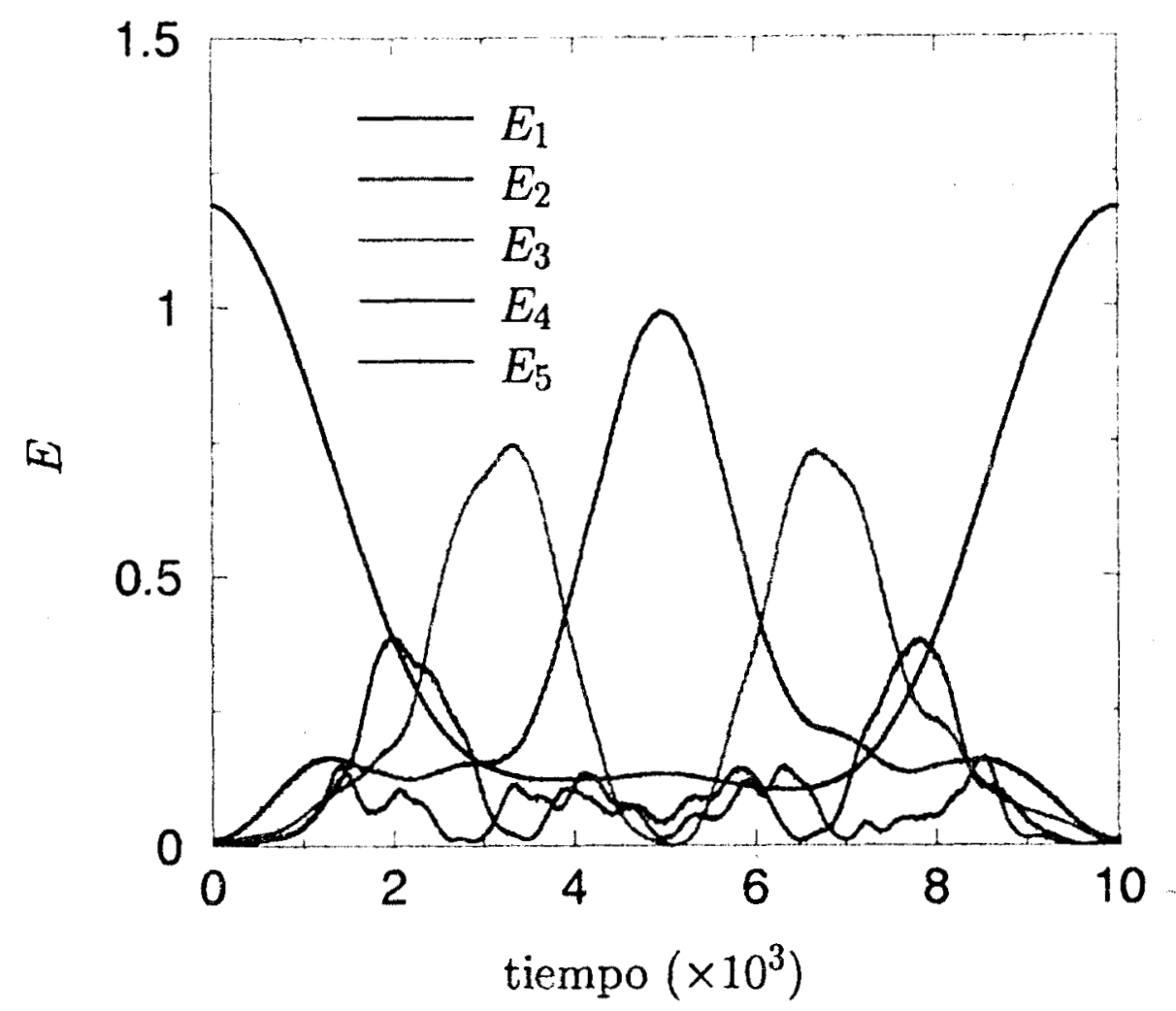

Figura 4.1: Evolución temporal de la energía $E_{i}$ de los primeros 5 modos normales. Reproducción de la figura 1 de la Ref. [57] utilizando el procedimiento numérico explicado en el apéndice D. En esta figura (y en todo el resto del trabajo) el tiempo está expresado en unidades naturales. Ver capítulo 5.

\subsubsection{Relevancia Física del Modelo FPU}

En vista de los resultados obtenidos por Fermi et al., varias preguntas surgen inmediatamente, cuyas respuestas tienen una importancia crucial para desarrollos posteriores. La primera de ellas concierne a la validez del modelo teórico propuesto como modelo explicativo de un sistema físico real. En primer lugar notemos que el sisterna definido por el Hamiltoniano FPU (4.1 ó 4.5) es un modelo aproximado del comportamiento de un sólido cristalino a bajas temperaturas. La reducción de complejidad en comparación a la situación física real es considerable, de igual manera que en el caso del movimiento Browniano tratado en el capítulo anterior. Sólo se considera una dimensión espacial y la interacción entre partículas (usualmente del tipo Lennard-Jones) es desarrollada en pequeños desplazamientos alrededor 
de las posiciones de equilibrio de las partículas. Esto equivale a considerar sólo el caso débilmente anarmónico (la situación presente a bajas temperaturas). Ahora. bien, en la actualidad podemos considerar que la relevancia fisica del modelo FPU ya ha sido firmemente establecida, pues ahora se sabe que diferentes modelos en una y dos dimensiones comparten una misma fenomenologia (ver [59] para una revisión). En particular, practicamente todos los modelos hasta ahora estudiados muestran un comportamiento no-estadístico para energías bajas (correspondientes a bajas temperaturas), mientras que a energías más altas la equipartición ocurre según lo esperado. Un punto importante a recordar es que la transición entre estos dos comportamientos ocurre en un intervalo de energías físicamente relevante. Por ejernplo, para un modelo de osciladores bidimensionales con interacciones de tipo Lennard-Jones [60], la transición, utilizando los parámetros físicos del argón, ocurre para temperaturas de $5 \% \mathrm{~K}$. Algunos cálculos recientes de dinámica molecular en un modelo de un cristal de xenon con impurezas diluidas consistentes en moléculas de yodo muestran que el tiempo requerido para que se dé la equipartición de la energía entre las moléculas de $I_{2}$ inicialmente excitadas y la matriz de Xe se incrementa abruptamente por debajo de los $30^{\circ} \mathrm{K}$; esta situación podría investigarse experimentalmente para una muestra real [61].

La segunda pregunta concierne a la posibilidad de observar la falta de equipartición entre grados de libertad en un experimento de laboratorio, no en una simulación de computadora. Si la respuesta es positiva y se encuentra además que el sistema experimental eventualmente relaja hacia el equilibrio, entonces el estudio del mecanismo responsable podría sugerir una posible explicación al problema de la equipartición en el modelo FPU. Una confirmación en este sentido la proporciona el cálculo de las propiedades termodinámicas del hidrógeno molecular $\mathrm{H}_{2}$ [62]. El hidrógeno que sólo tiene niveles rotacionales pares se conoce corno para-hidrógeno, y aquel que sólo tiene niveles impares, orto-hidrógeno. Se sabe además que, en una mezcla de orto- y para-hidrógeno en equilibrio, la composición de ambas especies es diferente dependiendo de la temperatura a la que esté la mezcla. Ahora bien, si se prepara una muestra de hidrógeno a temperatura ambiente y posteriormente es enfriada para realizar mediciones a baja temperatura de su calor específico, se encuentra una aparente discrepancia entre los resultados experimentales y las predicciones teóricas. La razón es que la conversión entre orto- y para-hidrógeno es extremadamente lenta, por lo que la composición a temperatura ambiente persiste a bajas temperaturas. Si se espera un tiempo lo suficientemente grande como para que el sistema alcance el estado de equilibrio 
correspondiente a bajas temperaturas y se vuelve a realizar la medición del calor específico, entonces se obtiene un excelente ajuste entre los datos experimentales y el cálculo teórico. Es claro entonces que la escala temporal de observación afecta dramáticamente los resultados obtenidos. Este "congelamiento" de grados de libertad es cualitativamente similar al que ocurre en el modelo FPU y permite suponer que la escala de tiempo en la que ocurre la equipartición de energía entre los modos normales es extremadamente grande para las condiciones originalmente usadas por Fermi et al. Aunque es una suposición bastante razonable y natural para resolver el problema, no ha sido nada trivial confirmarla, como veremos más adelante.

\subsubsection{Resultados Posteriores}

Para el caso específico del modelo FPU, Izrailev y Chirikov [63] fueron los primeros en mostrar que, para energías lo suficientemente altas, este modelo relaja hacia el estado de equipartición en escalas de tiempo cada vez más cortas conforme aumenta la energía del sistema. Este fenómeno sugirió la existencia de un umbral de equipartición $E_{e}$ tal que, para $E<E_{e}$, la equipartición no ocurre (o bien, ocurre para tiempos extraordinariamente grandes), mientras que, para $E>E_{e}$, la equipartición ocurre según lo esperado. Ahora bien, para que este comportamiento tenga relevancia dentro del contexto de la mecánica estadística, el umbral $E_{e}$ debe de persistir en el límite termodinámico $(N \rightarrow \infty)$. Tendría entonces que cumplirse $E_{e} \propto N$ para obtener el escalamiento $\lim _{N \rightarrow \infty} E_{e} / N=$ cte $>0$. Utilizando una representación del sistema en términos de los modos normales lineales y la excitación de un subconjunto de éstos que corresponde a las condiciones iniciales de la simulación original de Fermi et al., estos autores encontraron, por medio de aproximaciones analíticas, un umbral de equipartición finito en el límite termodinámico. Sin embargo, debido en parte a la falta de claridad en la definición del umbral pero sobre todo a sus importantes implicaciones, este resultado difícilmente pudo tomarse como concluyente.

Bocchieri et al. [64] observaron numéricamente el umbral de equipartición en un sistema de osciladores con interacciones tipo Lennard-Jones, aunque para valores muy pequeños de $N$ (entre 8 y 100). Resultados posteriores obtenidos por Livi et al. parecieron confirmar que $E_{e} \propto N$ en los modelos $\beta$ [65] y $\alpha$ [66] para valores de $N$ entre 64 y 512. Para obtener sus resultados, los autores anteriormente citados 
definieron una función, llamada entropía espectral, cuya forma explícita es

$$
S(t)=-\sum_{i=1}^{N / 2} p_{i}(t) \ln p_{i}(t),
$$

donde $p_{i}(t)=E_{i}(t) / \sum_{k=1}^{N / 2} E_{k}(t)$ y $E_{k}$ es la energía total del $k$-ésimo modo normal. Resulta entonces que $S$ alcanza su máximo valor, $S_{\max }=\ln (N / 2)$, cuando el sistema está en el estado de equipartición. Para eliminar la dependencia de $\mathcal{S}$ con respecto a $N$ se define a la entropía normalizada como

$$
\eta(t)=\frac{\mathcal{S}(t)-\mathcal{S}_{\max }}{\mathcal{S}(0)-\mathcal{S}_{\max }}
$$

de tal manera que $\eta \rightarrow 1$ cuando $S(t) \approx S(0)$ ("congelamiento" de las condiciones iniciales) y $\eta \rightarrow 0$ cuando $\mathcal{S}(t) \rightarrow \mathcal{S}_{\max }$ (equipartición). Cabe mencionar que ésta ha sido una cantidad fundamental en el estudio del intercambio de energía entre modos normales en el modelo FPU.

Sin embargo, incluso con la ayuda de $\eta(t)$, la dependencia de $E_{e}$ con respecto a $E, N$ y las condiciones iniciales no es nada trivial de estudiar. Para el modelo $\alpha$ se tiene que, si $E$ es muy grande, la parte cúbica del potencial se vielve repulsiva, obteniéndose soluciones no acotadas que provocan problemas de estabilidad. Debido a esto es que el modelo $\beta$ ha sido el más exhaustivamente estudiado hasta la fecha. Pettini y Landolfi [67] exploraron numéricamente la dependencia del tiempo $\tau_{e}$ en que el sistema alcanza el estado de equipartición como función de la energía para valores relativamente altos de ésta y con valores de $N$ entre 128 y 512 , obteniendo $\tau_{e} \propto N$ y un umbral de equipartición $E_{e} \propto N$, con una transición gobernada por una aproximación de matriz estocástica. Sin embargo, la equipartición presente a energías muy bajas y en escalas de tiempo mucho más lentas no fue estudiada. Kantz, Livi y Ruffo [68], también numéricamente, obtuvieron la misma dependencia del tiempo de equipartición con respecto al número de osciladores, pero para valores de $N$ entre 32 y 1024 . Más precisamente, considerando la dependencia funcional de $\eta$ con respecto a la energía $E$, el tiempo $t$ y el número de osciladores $N$, estos autores encontraron que $\eta$ depende sólo de dos variables, $E$ y $t / N: \eta(E, t, N)=f(E, t / N)$ para condiciones iniciales en las que toda la energía inicial se concentra en un grupo de modos normales $\Delta n$ tal que $\Delta n \propto N$ (condiciones iniciales "físicas"). Además, para condiciones iniciales en las que se excita a un subconjunto $\Delta n$ de modos normales tal que $\Delta n \ll N$ (condiciones iniciales "mecánicas") se obtiene $\eta(E, t, N)=g(E / N, t / N)$. Este último resultado 
parece estar en contradicción con los de De Luca, Lichtenberg y Lieberman [69] obtenidos analíticamente a partir de una representación efectiva del Hamiltoniano en términos de un conjunto reducido de modos normales y que parecen sugerir $\eta(E, t, N)=g\left(E, t / N^{2}\right)$; pero si ambos resultados tienen que ser válidos, entonces $\eta$ debe de ser función de un sólo parámetro, $E t / N^{2}$. Cálculos numéricos posteriores [70] sugieren una corrección adicional a esta última estimación, dando una dependencia de $\eta$ en términos de un solo parámetro: $\beta E n t /\left(N^{2} \sqrt{N}\right)$. Un resultado importante de este último trabajo es que incluso en el límite termodinámico con $E / N=$ cte $<1$ y $\Delta n \propto N$, el tiempo de equipartición diverge como $\sqrt{N}$. Esto último es muy intrigante puesto que sugiere que pueden persistir estados de no-equipartición para tiempos grandes si se efectúa el límite termodinámico $N \rightarrow \infty$ antes que el límite asintótico $t \rightarrow \infty$.

Los resutados anteriores para el modelo $\beta$ parecen indicar firmemente que el umbral de equipartición desaparece en el límite termodinámico. La conjetura de que este comportamiento es universal parece confirmada por estudios similares realizados con otros modelos no-armónicos. En particular, para el modelo $\phi^{4}$, el cual es semejante al modelo $\beta$ pero con un potencial anarmónico de la forma $U_{A N} \equiv \sum_{i}\left(\frac{1}{2} \lambda x_{i}^{2}+\frac{1}{4} \mu x_{i}^{4}\right)$, existe evidencia de que el sistema alcanza el estado de equilibrio en el límite termodinámico y que el tiempo de equipartición también diverge cuando el parámetro de anarmonicidad tiende a cero (esto es, en el límite armónico), aunque hasta el presente no ha sido posible determinar la naturaleza de esta divergencia (exponencial, polinomial o de algún otro tipo) [71]. Finalmente, para el modelo $\alpha$, Casetti et al. [72] han determinado, integrando las ecuaciones de movimiento para tiempos muy grandes $\left(\sim 10^{8}\right)$, un umbral $E_{e} \sim N^{-1}$ debajo del cual es posible inferir la eventual relajación del sistema hacia el estado de equipartición, pero para tiempos inobservables en cualquier computadora actual si se utilizan los mismos parámetros que originalmente usaron Fermi et al. Más específicamente, estos autores determinaron que, si en el estudio original de 1954 se hubiera utilizado una amplitud $A$ (ver Ec. 4.4) diez veces mas grande que la originalmente usada para establecer las condiciones iniciales, se hubiera podido observar la equipartición de energía según lo esperado.

Para concluir esta sección recordemos que los términos potenciales nocuadráticos en los Hamiltonianos (4.1) y (4.5) son los responsables del complicado mecanismo de intercambio de energía entre los modos normales (en la ausencia de estos términos el intercambio no ocurriría en lo absoluto, como ya mencionamos). $\mathrm{El}$ efecto de estos términos en las soluciones a las correspondientes ecuaciones de 
movimiento es considerable, pues la dinámica del sistema en el espacio fase es mucho más cornplicada. Ahora bien, desde hace mucho tiempo se ba especulado. que la presencia de trayectorias no-periodicas es una condición indispensable en el surgimiento de las propiedades estadísticas del sistema (equipartición de energía en el caso del modelo FPU). Por lo tanto, a continuación haremos una revisión de los resultados actualmente conocidos de la teoría general de los sistemas dinámicos cuando se tienen $N \gg 1$ grados de libertad, ya que este es el caso de interés en el contexto de la mecánica estadística.

\subsection{Dinámica de Sistemas Hamiltonianos}

El punto de vista más comunmente aceptado es que un estado macroscópico de equilibrio termodinámico corresponde a un conjunto de estados microscópicos cuya dinámica debe de cumplir con dos propiedades fundamentales: no debe de tener otras integrales de movimiento aparte de la energía y las correlaciones temporales de las variables dinámicas deben de decaer rápidamente. Dicho de otra forma, una órbita típica en el espacio fase, que representa el estado microscópico del sistema como función del tiempo, debe de moverse de forma aproximadamente aleatoria, o errática, sobre la hipersuperficie de energía constante determinada por las condiciones iniciales. Si uno desea que las correlaciones decaigan y que se pierda rápidamente la memoria del estado inicial, se requiere que cualesquiera dos órbitas que estén muy cerca la una de la otra en un instante dado, se separen rápidamente, perdiendo, en una escala de tiempo razonable, cualquier relación recíproca. Estas propiedades son características básicas de lo que actualmente se conoce como caos, entendido como lo opuesto a una dinámica ordenada, caracterizada por las siguientes propiedades: existencia de integrales de movimiento diferentes a la energía, memoria (persistencia) de las condiciones iniciales y un caracter regular (cuasi-periódico) de las órbitas en el espacio fase ${ }^{3}$.

Ahora bien, al problema del fundamento dinámico de la mecánica estadística clásica se le conoce como el problema ergódico, el cual es particularmente difícil de tratar. Esto es debido no sólo a que en sí es difícil de resolver, sino también porque no es fácil de formular apropiadamente. Pueden ser definidas diferentes

\footnotetext{
${ }^{3}$ Es conveniente hacer notar que la excepción a este punto de vista la constituyen los sistemas de osciladores armónicos estudiados en el capítulo anterior. El elemento estadístico está dado por las condiciones iniciales y el gran número de grados de libertad involucrados, no por la dinámica del sistema, que es totalménte regular.
} 
propiedades ergódicas [73], pero hasta el momento no es claro cuáles de estas propiedades son apropiadas para sustentar el enfoque mecanico-estadístico; esto es, no hay un método general disponible para determinar si un sistema es ergódico o no a partir de su Hamiltoniano, con la excepción del caso de una partícula libre que rebota elásticamente con las paredes del así llamado billar de Sinai [74]. Esta dificultad ha provocado que existan opiniones diferentes y controversiales sobre esta cuestión. Un punto de vista muy extendido ba negado desde hace mucho la existencia de este problema [75], considerando la imposibilidad de una construcción dinámica de los ensambles estadísticos; en el límite termodinámico el problema ergódico debería de pertenecer al ámbito de la teoría de las probabilidades [76]. El primer intento de obtener directamente un comportamiento estadístico a partir de la dinámica microscópica fue el trabajo ya reseñado de Fermi et al. cuyos resultados controversiales replantearon completamente el estudio del problema ergódico, ya que parecieron indicar la persistencia de un comportamiento cuasi-periódico incluso en presencia de un potencial anarmónico en el Hamiltoniano. Ahora bien, casi al mismo tiempo fue planteado el teorema de estabilidad de Kolmogorov para sistemas dinámicos de $N$ grados de libertad, el cual ofrece una posible interpretación a la falta de equipartición observada en el modelo FPU, como lo hicieron notar posteriormente Izrailev y Chirikov [63]. Es por esto que a continuación daremos una descripción (de ninguna manera exhaustiva) de los resultados teóricos actualmente conocidos para los sistemas dinámicos con $N \gg 1$ y de su posible relevancia para el estudio del problema planteado por el modelo FPU. Respecto al problema ergódico en general sólo estaremos interesados en aquellos puntos directamente relevantes para el estudio de este modelo en particular.

\subsubsection{Teoremas Generales}

Cuando un sistema dinámico de $N$ grados de libertad admite la existencia de $N$ integrales de movimiento y, como consecuencia de las leyes de conservación resultantes, tiene un movimiento acotado, se pueden definir las coordenadas canónicas conjugadas de acción-ángulo $(I, \theta)$ tales que $I=\left(I_{1}, \ldots, I_{N}\right) \in \mathcal{B} \subset \Re^{N}$ y $\theta=\left(\theta_{1}, \ldots, \theta_{N}\right) \in \mathbf{T}^{N}$. $\mathcal{B}$ es un subconjunto abierto de $\Re^{N}$, mientras que $\mathbf{T}^{N}$ denota a un toroide (subvariedad) $N$-dimensional, de tal manera que el Hamiltoniano del sistema es independiente de los ángulos, esto es

$$
H(\mathbf{I}, \theta)=H_{0}(\mathbf{I})
$$


Con este conjunto de coordenadas es inmediato ver que la dinámica del sistema es trivial, pues

$$
\mathbf{I}(t)=\mathbf{I}(0), \quad \theta(t)=\theta(0)+\omega(\mathbf{I}(0)) t,
$$

donde $\omega=\left(\omega_{1}, \ldots, \omega_{N}\right)=\partial H_{0} / \partial \mathbf{I}$ es el vector de frecuencias angulares. Entonces el espacio fase $\mathcal{B} \times \mathrm{T}^{N}$ está "foliado" en conjuntos de toroides $\mathbf{I} \times \mathrm{T}^{N}, \mathbf{I} \in \mathcal{B}$ de tal modo que una orbita que empiece en un toroide dado permanece para siempre en él. Los sistemas con esta propiedad se conocen como sistemas dinámicos integrables, siendo los más ordenados o "menos ergódicos". Notemos también que el modelo FPU sin el término anarmónico resulta ser un sistema de osciladores armónicos desacoplados (modos normales) que cumple con todas las propiedades arriba mencionadas, por lo que el Hamiltoniano independiente de los ángulos resulta ser $H_{0}(\mathbf{I})=\omega \cdot \mathbf{I} \equiv \sum_{i=1}^{N} \omega_{i} I_{i}$.

Sin embargo, muchos sisternas de interés están descritos por Hamiltonianos de la forma

$$
H(\mathbf{I}, \theta, \sigma)=H_{0}(\mathbf{I})+\sigma H_{1}(\mathbf{I}, \theta)+\sigma^{2} \ldots,
$$

por lo que surge de inmediato una pregunta natural ¿Será la dinámica del sistema perturbado, para $\sigma$ pequeña, en algún sentido parecida a la dinámica noperturbada o existen nuevas características producidas por la perturbación? Esta no es una pregunta nueva, ya que viene a ser una generalización del estudio sobre la equipartición que estudiamos en la sección anterior para el modelo FPU. De hecho, el Hamiltoniano (4.1) tiene la misma estructura que (4.10) hasta $O(\sigma)$. Ahora bien, a pesar de los grandes avances conseguidos, hasta la fecha el comportamiento general de (4.10) no es completamente claro si uno está interesado en sistemas con un número infinito de grados de libertad; esto es, en sistemas mecanico-estadísticos.

Consideremos otra vez el Hamiltoniano (4,10). El objetivo de la teoría clásica de perturbaciones sería el de encontrar (para $\sigma$ pequeña) un cambio de variables canónico $(\mathbf{I}, \theta)=\mathcal{C}_{\sigma}\left(\mathbf{I}^{\prime}, \theta^{\prime}\right)$ tal que el nuevo Hamiltoniano $H^{\prime}\left(\mathbf{I}^{\prime}, \theta^{\prime}, \sigma\right) \equiv$ $H\left(\mathcal{C}_{\sigma}\left(\mathbf{I}^{\prime}, \theta^{\prime}\right), \sigma\right)$ todavía conserve la forma integrable

$$
H^{\prime}\left(\mathbf{I}^{\prime}, \theta^{\prime}, \sigma\right)=H_{0}^{\prime}\left(\mathbf{I}^{\prime}, \sigma\right) \text {. }
$$

En ese caso el sistema perturbado sería esencialmente la imagen de aquel noperturbado: se tendrían trayectorias cuasi-periódicas en todo el espacio fase, sin comportamiento estadístico alguno. Esta posibilidad queda eliminada debido al teorema de Poincaré-Fermi [77], el cual establece que, para sistemas no-lineales 
con un número $N \geq 3$ no existen constantes de movimiento además de la energía del sistema. Esto quiere decir que no pueden existir subvariedades que separen al espacio fase en regiones disjuntas, como en el caso integrable.

Sin embargo, para perturbaciones pequeñas se tiene que las diferencias cualitativas entre los sistemas perturbado y no perturbado están confinadas a un subconjunto del espacio fase de medida despreciable, aunque abierto y denso. Además, incluso dentro de este subconjunto las acciones se comportan como integrales de movimiento aproximadas para tiempos extremadamente grandes. Estas afirmaciones provienen de un teorema fundamental establecido por Kolmogorov [78], el cual fue posteriormente ampliado y generalizado por Arnold [79] y Moser [80], de ahí que se conozca como "teorema KAM". Este teorema es válido para sistemas descritos por Hamiltonianos cuasi-integrables de la forma

$$
H(\mathbf{I}, \theta, \sigma)=H_{0}(\mathbf{I})+\sigma H_{1}(\mathbf{I}, \theta, \sigma), \quad \sigma \frac{\left\|H_{1}\right\|}{\left\|H_{0}\right\|} \ll 1,
$$

donde $\|$... $\|$ es una norma adecuada.

El teorema KAM establece que, si $0 \leq \sigma \leq \sigma_{c}$ (donde $\sigma_{c}$ depende del número de grados de libertad y de otros detalles del Hamiltoniano), entonces existe una transformación canónica $(\mathbf{I}, \theta)=\mathcal{C}_{\sigma}\left(\mathbf{I}^{\prime}, \theta^{\prime}\right)$ y un sistema dinámico $H_{0}^{\prime}\left(\mathbf{I}^{\prime}, \theta^{\prime}\right)$, tales que el nuevo Hamiltoniano $H^{\prime}\left(\mathbf{I}^{\prime}, \theta^{\prime}, \sigma\right) \equiv H\left(\mathcal{C}_{\sigma}\left(\mathbf{I}^{\prime}, \omega^{\prime}\right), \sigma\right)$ satisface la relación

$$
H^{\prime}\left(\mathbf{I}^{\prime}, \theta^{\prime}, \sigma\right) \stackrel{B_{g}}{=} H_{0}^{\prime}\left(\mathbf{I}^{\prime}, \sigma\right)
$$

donde $\mathcal{B}_{\sigma}$ es un subconjunto cerrado de $\mathcal{B}$ y el símbolo $\stackrel{\mathcal{B}_{\sigma}}{=}$ denota igualdad entre los dos miembros, así como de sus derivadas, para $I^{\prime} \in \mathcal{B}_{\sigma}$.

De la igualdad (4.13) se sigue que $I_{1}^{\prime}, \ldots I_{N}^{\prime}$ son integrales de movimiento (y, por lo tanto, la correspondiente órbita se mueve sobre un toroide invariante) si las condiciones iniciales pertenecen a $\mathcal{B}_{\sigma}$. Las acciones del sistema no-perturbado son integrales de movimiento aproximadas; esto es, se cumple $\left|I_{k}(t)-I_{k}(0)\right|=O(\sigma)$ para toda $t$. Debido a que el movimiento del sistema perturbado permanece similar al del sistema no-perturbado, se tiene que no es posible comportamiento estadístico alguno, excepto en el complemento de $\mathcal{B}_{\sigma}$.

Para el caso particular $N=2$ se tiene que los toroides invariantes son superficies bidimensionales contenidas en un "superficie" tridimensional de energía constante. Entonces, para cada "superficie" de energía, el complemento de $\mathcal{B}_{\sigma}$ está separado por los toroides en regiones disjuntas y de grosor exponencialmente 
pequeño en $\sigma$; una órbita que empiece en esta región en $t=0$ permanece atrapada en ella para cualquier tiempo posterior. Sin embargo, para $N>2$, se tiene que

$$
\text { dirn(superficie de energia constante })-\operatorname{dim}(\text { toroide })>1 \text {, }
$$

por lo que el complemento de $\mathcal{B}_{\sigma}$ está conectado. Queda entonces abierta la. posibilidad de tener un conjunto de órbitas moviéndose de manera errática sobre una "superficie" de energía. Este fenómeno se conoce como difusión de Amold y puede demostrarse que efectivamente ocure en algunos ejemplos sencillos [73]. Para sistemas de más de tres grados de libertad este mecanismo de "difusión" lenta ha sido frecucntemente propuesto como el responsable de hacer que una trayectoria dada atraviese todo el espacio fase accesible.

La eficiencia de este mecanismo lo determina el teorema de Nekhoroshev [81], el cual establece que existen constantes $a, A, \tau_{0}$ y $\nu$ tales que, si $\mathrm{I}(0)$ es el vector de las acciones iniciales, entonces se satisface que

$$
|\mathrm{I}(t)-\mathrm{I}(0)|<A \sigma^{a}
$$

al menos para tiempos $t \in[0, T]$, donde

$$
T \simeq \tau_{0} \exp \left[(1 / \sigma)^{\mu}\right]
$$

Además, este teorema es válido para perturbaciones lo suficientemente débiles tales que $0 \leq \sigma \leq \sigma_{c}$. Se sigue entonces que la trayectoria correspondiente a un sistema dado visita la mayor parte del espacio fase disponible en un tiempo exponencialmente grande $\sim \mathcal{O}(T)$, más grande de hecho que cualquier potencia negativa de $\sigma$. Para tiempos menores a $T$ existen trayectorias caóticas en el espacio complementario a los toroides, pero están acotadas e involucran sólo a algunos de los grados de libertad, los cuales permanecen descoplados del resto.

Después de la exposición anterior volvemos a preguntarnos por la posible relevancia del teorema KAM y del teorema de Nekhoroshev para el estudio de problemas de interés a la mecánica estadística. Una respuesta positiva implicaría que el umbral $\sigma_{c}$, así como los exponentes a y $\nu$ fuesen independientes de $N$. Sin embargo, todas las estimaciones que hasta el momento existen dan como resultado una dependencia muy pronunciada con respecto a $N$, como a continuación veremos. Esto hace que estos teoremas, a pesar de su relevancia teórica de caracter fundamental, sean de poca utilidad práctica para el estudio de sistemas de muchos grados de libertad como en los que estamos interesados. 
Con respecto al teorema KAM, las estimaciones de $\sigma_{c}(N)$ siempre dan una dependencia con respecto a $N$ de la forma $[82] \sigma_{c}(N) \sim \exp (-B N \ln N), B>0$, o bien [83] $\sigma_{c}(N) \sim N^{-\delta}$ con $\delta \approx 1300$ para algunos casos particulares. Es claro entonces que, para valores físicamente significativos de la perturbación y $N \gg 1$, las subvariedades estables (toroides KAM) pueden considerarse, en general, como de medida cero. El teorema de Nekhoroshev tampoco es muy útil cuando tenemos un sistema con muchos grados de libertad. El exponente $\nu$ que aparece en (4.16) tiene una dependencia muy pronunciada en $N$ : la estimación $\nu(N) \sim\left(1 / N^{2}\right)$ es del trabajo original [81], $\nu(N) \sim(1 / N)$ se obtuvo en [84] y también en [85], donde se establece que es una estimación óptima. Una prueba diferente del teorema de Nekhoroshev, basada en una estrategia totalmente distinta [86], da como resultado $(a, \nu)=\left(\frac{1}{2}, \frac{1}{2 N+3}\right)$ para los exponentes de las Ecs. (4.15) y (4.16). Esta última estimación también es óptima y prácticamente ya no hay posibilidades de mejoras subsecuentes para el caso general, aunque queda abierta la posibilidad de obtener alguna mejoría en las estimaciones anteriores para Hamiltonianos particulares.

\subsubsection{Resultados Analíticos Particulares}

Sin embargo, y a pesar de las estimaciones anteriores cuando $N \gg 1$, en el caso de sistemas de baja dimensionalidad tales como el modelo de Hénon-Heiles [87] se tiene que el teorema KAM proporciona una explicación satisfactoria de la fenomenología estudiada numéricamente. Para este caso particular se ha encontrado un umbral de caoticidad $E_{c}$ (el cual está relacionado a $\sigma$ a través de las condiciones iniciales) debajo del cual el sistema tiene un comportamiento cuasiperiódico, mientras que, para el caso contrario, su dinámica está dominada por órbitas caóticas. En la vecindad del umbral se tiene una coexistencia muy complicada de ámbos comportamientos. La pregunta relevante, al igual que en el caso del umbral de equipartición ya antes mencionado, vuelve a ser la de la persistencia de $E_{c}$ en el límite termodinámico.

Los pocos resultados analíticos disponibles para los modelos FPU- $\alpha$ y $\beta$ son bastante ambiguos debido a las muy importantes simplificaciones que deben de ser introducidas para obtener las estimaciones deseadas. Siguiendo la definición de umbral de inestabilidad exponencial en el modelo de Hénon-Heiles (el cual se obtiene a partir de (4.1) con $N=3$ y condiciones de frontera periódicas [88]), se ha obtenido una estimación de $E_{c}$ para el modelo FPU- $\alpha$ de la forma [89] $E_{c} \simeq 1 / 4 \alpha^{2}+O\left(1 / N^{2}\right)$, donde $\alpha$ es la constante de acoplamiento anarmónica. Se 
sigue entonces que la densidad crítica de energía $\epsilon_{c}=E_{c} / N$ desaparece en el límite $N \rightarrow \infty$. Para el modelo FPU- $\beta$, Izrailev y Chirikov [63] obtuvieron una ecuación de evolución no-lineal para la amplitud de un modo normal $n$ cualquiera, obteniendo un umbral $\epsilon_{c} \simeq 1 / \beta n$ independiente de $N$ ( $\beta$ es la constante de acoplamiento nolineal del modelo) para números de onda $n$ inicialmente excitados tales que $n \ll N$. En el lírnite opuesto, $N-n \ll N$, estos autores encontraron que $\epsilon_{c} \simeq \pi^{2} n^{2} / N^{4} \beta$, lo que implica que, para modos normales con númeras $n$ altos y $N$ grande, se obtiene un comportamiento estocástico inlcuso para una no-linealidad débil. Este último resultado concuerda con las estimaciones de Berman y Kolovskij [90] obtenidas por medio de una aproximación cotalmente diferente; además, estos autores encontraron que, debajo del umbral $\epsilon_{c}$, la dinámcia del modelo se puede describir por medio de una ecuación de Schrödinger no-lineal, la cual se sabe que es integrable. Todos estos enfoques quizás son muy simplificados, pero son los únicos intentos existentes hasta la fecha de tratar analíticamente el comportamiento caótico de sistemas Hamiltonianos con muchos grados de libertad.

\subsubsection{Resultados Numéricos}

Por el lado de los experimentos numéricos tenemos que el punto central es el de la elección de un indicador adecuado que pueda revelar la existencia del umbral de caoticidad $\epsilon_{c}$. Muchos parámetros han sido presentados en la literatura, aunque algunos de ellos están más relacionados con el problema de la equipartición que con la dinámica del sistema (suponen que la equipartición de energía implica inequívocamente la presencia de órbitas caóticas). Por ejemplo, se ha hecho notar que, si la entropia normalizada (4.7) se considera como una "variable dinámica", se pueden construir pseudo-secciones de Poincaré en el plano $(\dot{\eta}, \eta)$ que dan información cualitativa global sobre el intercambio de energía entre los modos normales [91]. Otros parámetros de este tipo son las funciones de autocorrelación de una partícula [92], parámetros relacionados a la estructura geométrica local del espacio fase [93] y métricas relacionadas al promedio temporal de la energía de los modos normales $[94]$.

Sin embargo, las cantidades que han resultado ser de mayor utilidad para el estudio del caracter caótico de las trayectorias en el espacio fase de un sistema dado son los llamados exponentes de Lyapunov. Su importancia radica en que cuantifican directamente la divergencia de dos trayectorias en el espacio fase inicialmente muy próximas (sensibilidad a las condiciones iniciales). Existe un exponente por 
cada dimensión del sistema, pero el que se usa regularmente como medida de caoticidad es el mayor de ellos [95], $\lambda_{1}$. Si $\lambda_{1} \gg 0$ se tiene inequívocamente una dinámica caótica, si $\lambda_{1}<0$ se tiene un comportamiento totalmente regular, y finalmente, si $0<\lambda_{1} \ll 1$, entonces se tiene una coexistencia de trayectorias ordenadas y caóticas. Para el modelo $\beta$ existe una fuerte evidencia numérica que hace plausible la existencia de una función de densidad para los exponentes de Lyapunov en el límite termodinámico [96]. La existencia de dicha función permite definir la entropía de Kolnogorov-Sinai (obtenida a partir de la suma de todos los exponentes de Lyapunov positivos del sistema) como una cantidad intensiva en el límite termodinámico, lo que resulta en una persistencia de trayectorias caóticas cuando el número de grados de libertad del sistema tiende a infinito. Desafortunadamente no existen resultados analíticos de caracter general (esto es, independientes de un modelo específico) que demuestren de manera inequívoca la existencia de la función de densidad ya antes mencionada, aparte de los obtenidos por Eckmann y Wayne [97] utilizando la aproximación de la matriz aleatoria.

Con respecto a los resultados obtenidos en el caso de modelos específicos, para el FPU- $\beta$ los resultados más importantes han sido obtenidos por Pettini y Landolf [67]. Además de comprobar que la dinámica del modelo está controlada por el parámetro intensivo ${ }^{4} \epsilon=E / N$, estos autores encontraron un umbral $\epsilon_{c}$ que no corresponde estrictamente a un umbral de equipartición (pues ésta siempre ocurre para tiempos suficientemente grandes) ni a un umbral de caoticidad (ya que ésta siempre está presente si $\beta \neq 0$ ), sino más bien a un umbral de caoticidad fuerte (SST por sus siglas en inglés). El SST es tal que, para $\epsilon>\epsilon_{c}$, ocurre una "difusión" rápida de una trayectoria dada en todo el espacio fase disponible, teniendo el máximo exponente de Lyapunov un escalamiento de la forma $\lambda_{1}(\epsilon) \sim \epsilon^{2 / 3}$, obtenido también para el modelo $\phi^{4}$ y que parece ser independiente del modelo usado. Esto último es debido a que el escalamiento de $\lambda_{1}$ implica una dinámica que semeja a un proceso aleatorio, lo cual fue a su vez comprobado por medio de una aproximación de matriz aleatoria. Por otra parte, para $\epsilon<\epsilon_{c}$, está presente un mecanismo de "difusión" mucho más lento, caracterizado por un rápido decremento de $\lambda_{1}$ conforme $\epsilon$ disminuye y que ocurre en una escala de tiempo cuya dependencia en $\epsilon$ está descrita por una expresión semejante a la de la difusión de Arnold, Ec. (4.16). Sin embargo, el correspondiente exponente $\nu$ que estos autores obtuvieron resulta ser independiente de $N$, por lo que resulta difícil de

\footnotetext{
${ }^{4}$ Este punto, que es de importancia crucial para el presente trabajo, se explicará con más detalle en el capítulo siguiente
} 
entender este mecanismo de relajación en el contexto del teorema de Nekhoroshev. Además, el umbral $\varepsilon_{c}$ resulta también ser independiente de $N$ para los valores estudiados (entre 32 y 128), por lo que la persistencia de este umbral en el línite termodinámico podría tener importantes implicaciones para los fenómenos estudiados por la mecánica estadística. Para el modelo FPU- $\alpha$ resulta imposible estudiar al SST ya que el potencial cúbico impide trabajar a energías muy altas. A pesar de esto, Casetti et al. [72] han podido definir un umbral de caoticidad para este modelo utilizando como criterio la persistencia del comportamiento $\lambda_{1} \sim t^{-1}$ (característico de una dinámica regular) hasta un tiempo máximo de $t=4.3 \times 10^{8}$. Esta es la primera evidencia directo de la existencia de un umbral de caoticidad que ha podido obtenerse para el caso $N \gg 2$. Sin embargo, y al igual que en el caso de la relación entre el modelo FPU- $\beta$ y el teorema de Nekhoroshev antes aludida, el escalamiento del umbral con respecto al número de osciladores, $\epsilon_{c} \sim N^{-2}$, no es explicable dentro del esquema del teorema KAM, pues en la estimación ya antes mencionada de $\sigma_{c} \sim N^{-\delta}$, el exponente es demasiado grande: $\delta \approx 1300$.

\subsection{Conclusiones}

En el presente capítulo hemos mostrado cómo un modelo undimensional de osciladores anarmónicos aparentemente muy sencillo puede desplegar un comportamiento dinámico extremadamente complejo que tiene un interés intrínseco, entre otras razones porque la fenomenología observada en experimentos numéricos no puede ser explicada satisfactoriamente por los teorernas hasta ahora conocidos (KAM y Nekhoroshev). Esto hace que la dinámica de este modelo (y otros muchos) sea todavía en gran parte desconocida cuando $N \gg 1$. Por lo tanto, las simulaciones numéricas siguen siendo una herramienta indispensable en el estudio de este tipo de sistemas, ya que sólo muy recientemente las aproximaciones analíticas han podido aplicarse con un grado razonable de éxito. Algunos de estos resultados incluyen la obtención de soluciones exactas de baja dimensionalidad [98] y la estimación del máximo exponente de Lyapunov por medio de la evolución temporal de un vector que representa la desviación entre geodésicas en una variedad Riemanniana construida con la métrica de Eisenhart sobre el espacio-tiempo $[99,100]$. También ha quedado en evidencia que la relación entre la dinámica Hamiltoniana y el comportamiento estadístico no es nada trivial ni es tan inmediata como podría pensarse. De hecho, en un estudio "termodinámico" [101] de varios modelos Hamiltonianos no-armónicos se ha descubierto que un sistema de rotores rígidos 
tiene un comportamiento no-ergódico para el calor específico en la misma escala temporal y en el mismo rango de energía para los cuales el modelo FPU muestra un comportamiento mecanico-estadístico normal para este mismo observable. Sin embargo, también es sabido que el modelo FPU no es capaz de reproducir la ley de Fourier [102]. Estos resultados implican que el comportamiento estadístico puede depender crucialmente del tipo de variable macroscópica que se esté estudiando, así como de la elección del modelo microscópico particular utilizado. Ahora bien, para el problema específico de la conducción de calor se ha hecho la observación [103] de que los resultados hasta ahora conocidos pueden ser de interés para describir sistemas experimentales tales como cristales fuertemente anisotrópicos [104], polímeros [105] y nanotubos [106]. A su vez, también han sido presentados algunos estudios teóricos sobre la conductividad térmica de un tubo cuántico en los regímenes balístico [107] y anarmónico [108] cuyos resultados podrían ser comprobados experimentalmente. Por lo tanto, puede concluirse que los modelos unidimensionales de muchos grados de libertad son lo suficientemente poderosos como para estudiar problemas físicamente relevantes; existe, sin em-. bargo, un fenómeno de gran interés mecanico-estadístico que todavía permanece inexplorado con este tipo de modelos: el movimiento Browniano. Esta tarea la emprenderemos en el capítulo siguiente. 


\section{Capítulo 5}

\section{El Modelo FPU Modificado}

\subsection{Introducción}

Como ya tuvimos ocasión de ver en el capítulo 3, los modelos de osciladores armónicos son los únicos que han permitido deducir de manera analítica, a partir del Hamiltoniano que los describe, la ecuación de Langevin, así como la fenomenología presentada en el capítulo 2. Sin embargo, son modelos sumamente restrictivos en cuanto al tipo de interacción entre osciladores se refiere, pues un potencial armónico no tiene la complejidad de aquellos utilizados en el estudio de fluidos, aunque, por otra parte, ofrece importantes ventajas en cuanto al esclarecimiento del tipo de suposiciones necesarias para pasar de una descripción microscópica Hamiltoniana a una de tipo estadístico. Por otra parte, el modelo FPU, que involucra interacciones más complejas que el caso armónico, pero que conserva la simplicidad de los modelos unidimensionales, ya ha sido ampliamente estudiado y es todo un paradigma en cuanto al estudio de sistemas no lineales con muchos grados de libertad se refiere.

En este capítulo construiremos un modelo de osciladores anarmónicos para el estudio del movimiento Browniano partiendo del Hamiltoniano (3.1) pero añadiendo un término potencial anarmónico del tipo FPU. De hecho, nuestro modelo resulta ser idéntico a este último, pero con un oscilador masivo en la posición central de la cadena. Aunque el comportamiento del modelo FPU no está entendido por completo como ya vimos en el capítulo 4, consideramos que lo conocido hasta ahora es suficiente como para aplicarlo al estudio del movimiento Browniano. Cabe mencionar que ya ha sido anteriormente estudiada la cadena FPU diatómica [109] y con una distribución aleatoria de masas para el caso de equipar- 
Capítulo 5. El Modelo FPU Modificado

tición [110] y transporte de energía [111], respectivarnente. Sin embargo, nuestra particular modificación al modelo FPU y su aplicación al estudio del movimiento Browniano es totalmente novedosa en el campo de la mecánica estadística de los modelos Hamiltonianos.

A diferencia del modelo de osciladores arnónicos anteriormente estudiado, para el nuestro ya no es posible encontrar soluciones analíticas a las ecuaciones de movimiento resultantes, por lo que tendremos que utilizar las técnicas de la dinámica molecular. Por medio de éstas estableceremos, primero, en qué intervalo de valores de los parámetros propios de nuestro modelo podemos obtener una fenomenología físicamente razonable. Posteriormente estudiaremos el comportamiento estadístico de auestro modelo para poder construir un estado de equilibrio termodinámico que finalmente nos permita comprobar si el oscilador pesado realiza movimiento Browniano. Esta última tarea, sin embargo, la emprenderemos hasta el capítulo siguiente.

\subsection{Planteamiento del Modelo}

Nuestro objetivo en la presente sección es plantear el Hamiltoniano de un sistema de osciladores anarmónicos de la forma $H=H_{0}+\sigma H_{1}$ en el cual $H_{0}$ es el Hamiltoniano para un sistema de osciladores armónicos acoplados (3.1) ya estudiado en el capítulo 3. Si en este último realizamos el cambio de índices $i \rightarrow i+N / 2$ tenemos que

$$
H_{0}=\sum_{i=0}^{N} \frac{P_{i}^{2}}{2 M_{i}}+\frac{\kappa}{2} \sum_{i=0}^{N}\left(X_{i+1}-X_{i}\right)^{2}
$$

donde $M_{i}=m$ si $i \neq 0$ y $M_{0}=M$. Las condiciones periódicas se escriben ahora como $X_{N+1}=X_{0}$. Ahora bien, aunque en principio tenemos la libertad de escoger la forma explícita de la parte anarmónica $H_{1}$, es importante tomar en cuenta algunas consideraciones. La más importante es que sería conveniente que $H_{1}$ corresponda a un modelo ya conocido para el caso de masas iguales, puesto que en ese caso se pueden hacer comparaciones directas con resultados ya publicados en la literatura, facilitando así el trabajo de validación que realizaremos posteriormente. Entonces los candidatos más viables resultan ser los modelos FPU estudiados en el capítulo anterior. De ellos elegiremos el que corresponde al potencial cuártico $\frac{1}{4} \sum_{i}\left(X_{i+1}-X_{i}\right)^{4}$ entre otras razones porque da origen a fuerzas puramente atractivas, propiedad que es compartida con el potencial armónico en 
$H_{0}$. Nuestro sistema queda descrito por el siguiente Hamiltoniano

$$
H=\sum_{i=0}^{N} \frac{P_{i}^{2}}{2 M_{i}}+\frac{\kappa}{2} \sum_{i=0}^{N}\left(X_{i+1}-X_{i}\right)^{2}+\frac{\sigma}{4} \sum_{i=0}^{N}\left(X_{i+1}-X_{i}\right)^{4}
$$

A continuación hacemos las transformaciones $M_{i}=m \widetilde{M}_{i}, P_{i}=\sqrt{m} \widetilde{P}_{i}$ y $X_{i}=$ $\widetilde{X}_{i} / \sqrt{\kappa}$, con lo cual $(5.2)$ se escribe como

$$
H=\sum_{i=0}^{N} \frac{\widetilde{P}_{i}^{2}}{2 \widetilde{M}_{i}}+\frac{1}{2} \sum_{i=0}^{N}\left(\widetilde{X}_{i+1}-\widetilde{X}_{i}\right)^{2}+\frac{\sigma}{4 \kappa^{2}} \sum_{i=0}^{N}\left(\widetilde{X}_{i+1}-\widetilde{X}_{i}\right)^{4}
$$

donde ahora tenemos que la masa de cada oscilador se ha convertido en un parámetro adimensional, puesto que $\widetilde{M}_{i}=1$ si $i \neq 0$ y $\widetilde{M}_{0}=\widetilde{M} \equiv M / m$. Por otra parte, el parámetro $\sigma / \kappa^{2}$ cuantifica la contribución de la parte anarmónica a la evolución del sistema. Como el trabajo subsecuente se hará con el Hamiltoniano (5.3), podemos, sin peligro de confusión, hacer el cambio de variable $\widetilde{X}_{i} \rightarrow X_{i}$, $\widetilde{P}_{i} \rightarrow P_{i}$ y definir $\beta \equiv \sigma / \kappa^{2}$, con lo que tenemos finalmente que

$$
H=\sum_{i=0}^{N}\left[\frac{P_{i}^{2}}{2 M_{i}}+\frac{1}{2}\left(X_{i+1}-X_{i}\right)^{2}+\frac{1}{4} \beta\left(X_{i+1}-X_{i}\right)^{4}\right],
$$

donde $M_{i}=1$ si $i \neq 0$ y $M_{0}=M$. Este último Hamiltoniano define un modelo que, por su semejanza formal con (4.5), lo llamaremos modelo FPU- $\beta$ modificado.

Las ecuaciones de movimiento que se obtienen a partir de (5.4) tienen la forma

$$
\frac{d^{2} X_{i}}{d t^{2}}=\left(1-\frac{M-1}{M} \delta_{i 0}\right)\left(X_{i+1}+X_{i-1}-2 X_{i}+F_{i}\left(\left\{X_{i}(t)\right\}\right)\right) \equiv G_{i}\left(\left\{X_{i}(t)\right\}\right),
$$

donde

$$
F_{i}\left(\left\{X_{1}(t)\right\}\right)=\beta\left[\left(X_{i+1}-X_{i}\right)^{3}-\left(X_{i}-X_{i-1}\right)^{3}\right] .
$$

Ahora bien, a diferencia de las ecuaciones (3.3) correspondientes al Hamiltoniano armónico (3.1), las ecuaciones (5.5) no son solubles analíticamente. Sin embargo, es perfectamente posible obtener soluciones numéricas con bastante precisión utilizando las técnicas de la dinámica molecular, lo cual mostraremos en detalle a continuación.

\subsection{Implementación y Validación del Modelo}

Las ecuaciones de movimiento (5.5) se integraron numéricamente utilizando el algoritmo de Verlet [112], el cual corresponde a las ecuaciones de diferencias finitas (D.5) y (D.15) para el cálculo de las posiciones y momentos, respectivamente. 
En el apéndice $D$ se hace una descripción detallada de cómo obtener ambas ecuaciones, así como una discusión de los problemas de implementación del algoritmo a nuestro modelo desde un punto de vista meramente numérico. En la presente sección nos ocuparemos de los problemas de implementación que están más directamente relacionados a la física del sistema.

Nuestro primer objetivo será el establecer las posiciones y momentos con la que vamos a inicializar nuestras simulaciones. Es claro que estas condiciones no pueden ser totalmente arbitrarias. Teniendo en cuenta que estamos trabajando esencialmente con un sólido cristalino y que desearnos estudiar el comportarniento del oscilador pesado cuando el baño de osciladores livianos esté en un estado de equilibrio termodinámico, las condiciones iniciales más fáciles de implementar tienen la forma

$$
X_{i}(0)=0 \quad P_{i}(0)=r_{i}, \quad i=0, \ldots, N
$$

las cuales corresponden a tomar a todos los osciladores del sistema en reposo (lo que tiene la considerable ventaja de preparar el estado inicial independientemente del valor de $\beta$ ) y donde $\left\{r_{i}\right\}$ son números aleatorios distribuidos Gaussianenente con media cero y varianza $\sqrt{2 M_{i}} \epsilon$, siendo $\epsilon$ la densidad de energía ya definida con anterioridad. Esta elección de momentos iniciales nos permite simular la situación física en la cual un cristal está preparado a una cierta temperatura (definida como la energía cinética promedio por grado de libertad) en equilibrio termodinámico. Para obtener los momentos $\left\{P_{i}\right\}$ primero se genera un conjunto de números aleatorios distribuidos Gaussianamente con media cero y varianza 1 y después se ajustan por medio de un reescalamiento que produzca como resultado un valor fijo de $\epsilon$. Se impone además la restricción $\sum_{i} P_{i}(0)=0$ para evitar un incremento sistemático en las variables espaciales. Aunque esta elección de los momentos $\left\{P_{i}\right\}$ es consistente con el valor de la temperatura de un cierto estado de equilibrio termodinámico, es claro que el sistema no empieza su evolución temporal en un punto del espacio fase correspondiente a ese estado de equilibrio, ya que el sistema está espacialmente ordenado al inicio de cada una de nuestras simulaciones. Podemos decir entonces que la parte del espacio fase que corresponde a los momentos está en equilibrio termodinámico, pero la parte que corresponde a las posiciones está fuera de equilibrio, siendo el estado total del sistema de este último tipo. Tenemos entonces que dejar que el sistema relaje hacia el estado de equilibrio total consistente con la temperatura que se establece a partir de los momentos. Los parámetros con los que estudiaremos la relajación hacia el equilibrio y las escalas de tiempo en la que ésta ocurre serán el tema de estudio 
de la siguiente sección.

Una última característica de las condiciones iniciales determinadas por (5.7) es que nos permiten fijar los valores de la densidad de energía con mucha precisión. Esto último es extremadamente importante porque es precisamente el valor de este parámetro intensivo el que controla el tipo de comportamiento que presenta nuestro modelo, de igual manera que con los modelos FPU $\alpha$ y $\beta$ reseñados en el capítulo anterior. Este hecho resulta de que, para una energía fija, la constante de acoplamiento no-armónica $\beta$ determina la contribución de la parte anarmónica del potencial a la dinámica definida por las ecuaciones de movimiento (5.5). Sin embargo, y como es más natural, para una $\beta$ fija la desviación del comportamiento armónico se obtiene al incrementar la energía del sistema. Para entender este punto con más claridad hacemos notar que las ecuaciones (D.5) y (D.15) que definen nuestro algoritmo de integración permanecen invariantes ante la transformación de escala $\widetilde{X}_{i}=\mu X_{i}, \widetilde{P}_{i}=\mu P_{i}$ y $\widetilde{\beta}=\mu^{-2} \beta$ mientras que la energía, ante esta misma transformación, tiene un escalamiento de la forma $\widetilde{E}(\widetilde{X}, \widetilde{P}, \widetilde{\beta})=\mu^{2} E(X, P, \beta)$. Por lo tanto, la dinámica del sistema, con una $\beta$ y $E$ o una $\widetilde{\beta}$ y $\widetilde{E}$, es exactamente la misma si se cumple la condición $\beta E=\tilde{\beta} \widetilde{E}$. Ahora bien, puede argüirse que, si $\beta \neq 0$, el sistema eventualmente manifestará un comportamiento caótico. Sin embargo ha podido demostrarse de manera numérica que, para el modelo FPU- $\alpha$ [72], la escala de tiempo $\left(t \sim 1.6 \times 10^{6}\right)$ en la que se manifiesta el comportamiento caótico cuando $\epsilon \approx 0.04 \ll 1$ es de al menos un orden de magnitud mayor que las que hemos utilizado para obtener la mayoría de nuestros resultados $\left(t_{\max } \sim 7 \times 10^{5}\right)$. Aunque la comparación es sólo cualitativa, pues los modelos son diferentes, podemos suponer, con un grado razonable de certidumbre, que este comportamiento es común en ambos modelos. De hecho, los resultados presentados en éste y el siguiente capítulo confirman satisfactoriamente nuestra suposición. Una ventaja adicional de adoptar este segundo enfoque proviene de que, como deseamos estudiar a nuestro sistema en el límite termodinámico $N \rightarrow \infty$, es conveniente definir un parámetro de control que sea independiente del número de osciladores $N$. Para el rnodelo FPU- $\beta$ de masas iguales y $\operatorname{con} \beta=0.1$ (valor que utilizaremos en todas nuestras simulaciones) se ha encontrado que $[113,114,115]$, cuando $\epsilon \geq 1$ estamos en una región del espacio fase en la que los efectos de la anarmonicidad son importantes para la dinámica del modelo. Por otra parte, cuando $\epsilon \ll 1$ estamos en una región del espacio fase en la que el efecto del término armónico en (5.4) es dominante, por lo que el comportamiento del sistema es regular (periódico): Cabría ahora preguntarnos si esta fenomenología es 
alterada de alguna forma al perturbar la cadena con un defecto pesado en el sitio $i=0$. A través del estudio de diversos indicadores dinámicos podremos contestar de manera negativa a esta pregunta, además de que nos permitirán comprobar la estabilidad de nuestro algoritmo de integración y fijar los valores de algunos parámetros de nuestro modelo antes de estudiar la relajación hacia el equilibrio termodinámico.

\subsubsection{Conservación de la Energía}

Debido a que el Hamiltoniano (5.4) no depende explícitamente del tiempo, la energía total es una constante de movimiento, por lo que debe de permanecer invariante ante la evolución temporal del sistema. El algoritmo de Verlet es simpléctico, lo que significa que el esquema definido por (D.5) preserva las áreas en el espacio fase y garantiza una representación local adecuada del flujo Hamiltoniano. Una consecuencia inmediata es que la conservación de la energía total del sistema puede obtenerse numéricamente con un muy alto grado de precisión. Esta propiedad del algoritmo es importante porque es bien sabido que, para sistemas Hamiltonianos con un número muy grande de grados de libertad, ésta es la única prueba de precisión que puede implementarse de manera sencilla. A continuación tenemos que elegir un paso de integración $\Delta t$ lo suficientemente pequeño para que nuestro esquema de integración preserve el flujo Hamiltoniano $y$, por lo tanto, conserve adecuadamente la energía inicialmente proporcionada al sistema. Sin embargo, y debido en gran medida al número de osciladores $N_{\max } \sim O\left(10^{5}\right)$ necesarios para obtener los resultados reportados en el capítulo siguiente, no se puede tomar un $\Delta t$ muy pequeño, ya que los cálculos se volverían prohibitivamente largos. Nuestra estrategia consistió entonces en hacer varias simulaciones con $N_{m a x}$ y diferentes pasos de integración para un amplio rango de valores de $\epsilon$ de tal manera que siempre se mantenga el mismo número de cifras significativas en el cálcuio de la densidad de energía. Siguiendo esta metodología se encontró que, con $\Delta t=0.05$ (que corresponde aproximadamente a un sexagésimo del tiempo más rápido de la parte armónica de la cadena, $t_{\min }=\pi$ y que está relacionado con el tiempo de interacción microscópico mencionado el el capítulo 3), se mantiene un número constante de 5 cifras significativas para valores de $\epsilon$ entre 0.01 y 10 , los cuales son valores representativos de los regímenes armónico y anarmónico, respectivamente, para una cadena FPU de masas iguales.

En la figura 5.1 graficamos la densidad de energía cinética, potencial y tota! 
$\frac{3}{\mid x}$

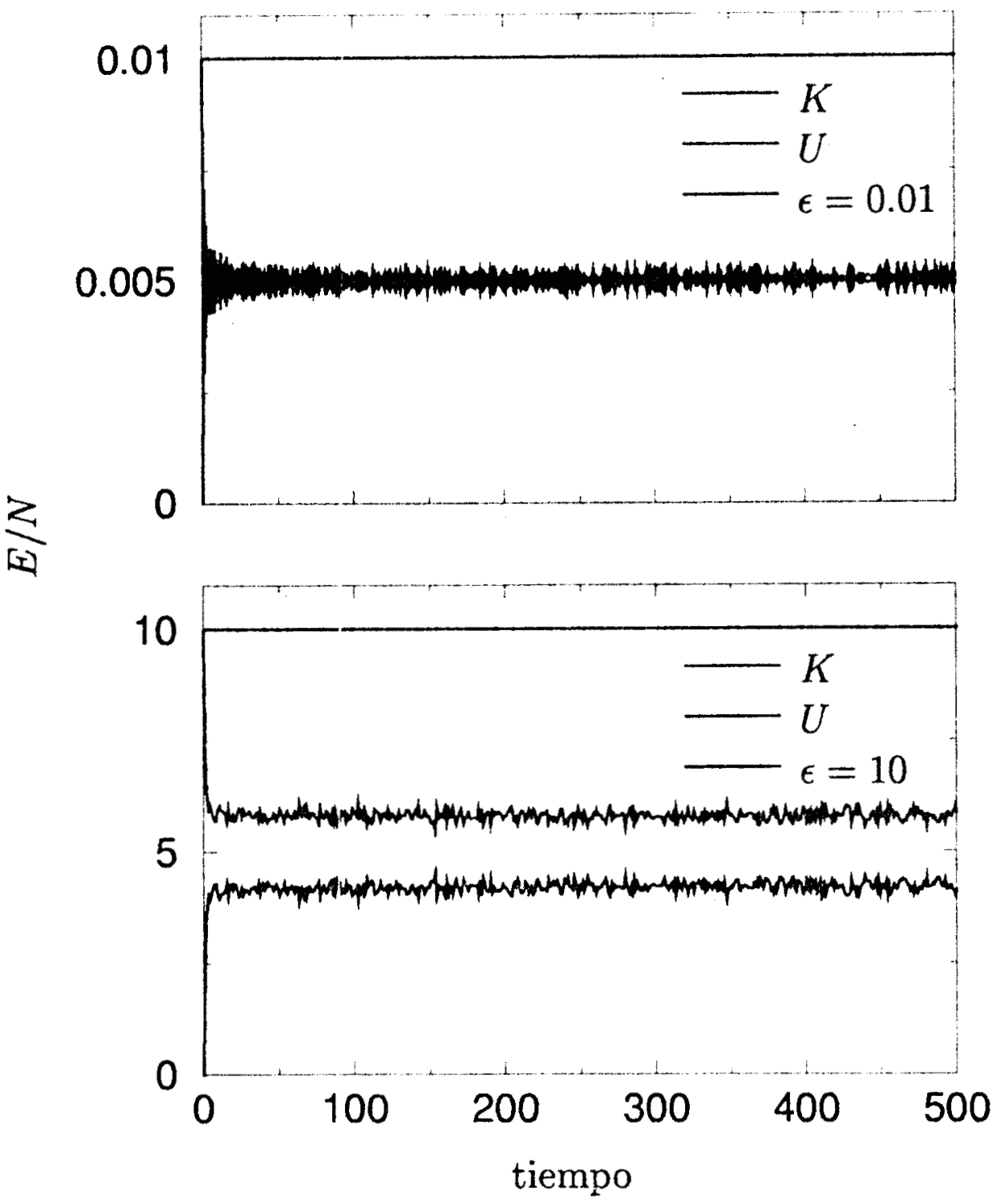

Figura 5.1: Densidad de energía cinética $K$, densidad de energía potencial $U$ y densidad de energía total $\epsilon$ vs tiempo para $M=60$ y $N=2000$. Se presentan dos casos que corresponden al régimen armónico $(\epsilon=0.01)$ y caótico $(\epsilon=10)$. Si se dividen los valores del eje horizontal entre $\pi$ se obtiene el tiempo expresado en unidades del período más rápido de la parte armónica del Hamiltoniano (5.4), $t_{\min }=\pi$. 
contra el tiempo $t$ (en unidades naturales) para dos valores distintos de la energía total inicialmente dada al sistema y con la masa del oscilador pesado igual a 60 . En ambos casos es claro que la conservación de la densidad de energía total es excelente para el intervalo de tiempo mostrado. Estos resultados son indistinguibles de aquellos obtenidos con $\Delta t=0.025$ y 0.0125 . En la escala de las figuras la densidad de energía total parece ser constante; sin embargo, existen fluctuaciones que provienen de la discretización de las ecuaciones de Newton. Pero estas fluctuaciones son de al menos un orden de magnitud menor que las fluctuaciones $\sim O\left(N^{-1}\right)$ de las energías cinética y potencial. Y lo que es más importante, la energía total no muestra ninguna desviación sistemática incluso para tiempos que corresponden a más de 20 millones de pasos de integración (con $\Delta t=0.05$ ). Tampoco se observó que estos resultados dependieran del valor específico de $M$ en un intervalo de entre 1 y 100.

Por lo demás vale la pena observar que, para el caso de $\epsilon=0.01$, las densidades de energía cinética y potencial son aproximadamente de la misma magnitud. Esto no es otra casa que la manifestación del hecho, bien conocido de la mecánica estadística, que la energía cinética promedio de una cadena armónica es la mitad de la energía total. Tenemos entonces una primera evidencia empírica directa de que la densidad de energía total es el parámetro que controla la no-linealidad del sistema puesto que, para $\epsilon=10$, la densidad de energía cinética es una fracción mayor que la densidad de energía potencial, siendo este un comportamiento que ya no es de caracter armónico.

\subsubsection{Parámetro de Control}

El intervalo de valores de $\epsilon$ en el que se presenta la transición de un comportamiento armónico a uno anarmónico permanece en gran medida inalterado por la presencia del defecto en la cadena. Pero la información asintótica que pueden proporcionar las energías cinética y potencial instantáneas es muy pobre, además de que la relación entre la evolución temporal de estas cantidades y la dinámica subyacente no es muy inmediata, por lo que sería deseable tener un indicador más sensible que pudiera determinar inequívocamente (y de manera independiente) el tipo de comportamiento que presente el sistema para un cierto valor de $\epsilon$. Esta tarea no es nada fácil, puesto que el estudio dinámico de los sistemas multidimensionales es mucho más difícil que la de sistemas tales como el de Hénon-Heiles [87] con $N=2$. Para estos últimos existen una serie de indicadores muy precisos y 
ampliamente utilizados (tales como las secciones de Poincaré), pero que, en gran medida, no son aplicables para el caso de sistemas con $N \gg 1$. Sin embargo, y teniendo en cuenta que nuestro objetivo es sólo una comprobación de la relevancia de la fenomenología ya conocida del modelo FPU- $\beta$ usual a nuestro problema particular, resulta que el estudio de la distancia entre dos trayectorias en el espacio fase inicialmente separades por una distancia infinitesimal nos proporciona información bastante detallada sobre la estructura de ese espacio, además de ser muy fácilmente implementable desde el punto de vista computacional. La distancia entre dos trayectorias se define explícitamente como

$$
d(t)=\left\langle\left\langle\left(\sum_{i=0}^{N}\left[\left(P_{i}^{\prime}(t)-P_{i}(t)\right)^{2}+\left(X_{i}^{\prime}(t)-X_{i}(t)\right)^{2}\right]\right)^{\frac{1}{2}}\right\rangle\right\rangle,
$$

siendo las condiciones iniciales $\left\{X_{i}(0), P_{i}(0)\right\}$ y $\left\{X_{i}^{\prime}(0), P_{i}^{\prime}(0)\right\}$ de ambas trayectorias idénticas, salvo por $X_{0}^{\prime}(0)=X_{0}(0)+10^{-6}$. El promedio $\langle\langle\cdots\rangle\rangle$ se toma sobre diez pares de trayectorias distintas para evitar las fluctuaciones que surgen de una elección particular de las condiciones iniciales, las cuales se obtuvieron a partir de una distribución Gaussiana, como ya lo mencionamos anteriormente. La distancia $d(t)$ se calculó en un intervalo de 200 unidades de tiempo para un conjunto de condiciones iniciales que corresponden a $\epsilon=0.01, \epsilon=10$ y algunos valores intermedios, mientras que los valores de $M$ se escogieron en un intervalo de entre 1 y 100 .

Los resultados para los casos $M=1$ y $M=60$ están reportados en la figura 5.2. En ambos casos es evidente que, para las trayectorias que empiezan en las regiones del espacio fase con $\epsilon \ll 1$, la distancia entre las trayectorias no cambia de orden de magnitud $\sim O\left(10^{-6}\right)$ para todo el intervalo de tiempo mostrado. Por lo tanto, podemos afirmar que el comportamiento del sistema es regular en estas regiones. Un escenario totalmente diferente se obtiene para $\epsilon \geq 1$, obteniéndose una divergencia de caracter exponencial que es característica de una dinámica dominada por la presencia de órbitas caóticas. En estos casos la distancia $d(t)$ aumenta en varios órdenes de magnitud (de $10^{-6}$ a $10^{2}$ aproximadamente) y, para el caso específico de $\epsilon=10$, se obtiene una separación máxima, puesto que las trayectorias están acotadas por el tamaño de la hipersuperficie de energía. En cuanto al efecto de la masa $M$ sobre los valores de $\epsilon$ correspondientes a las regiones regulares y caóticas del espacio fase es claro que, exceptuando una pequeña región en la que $t \ll 10$, el mismo es de poca consideración, como puede verse claramente para el caso $M=60$. Hemos además comprobado la estabilidad de estos resultados 


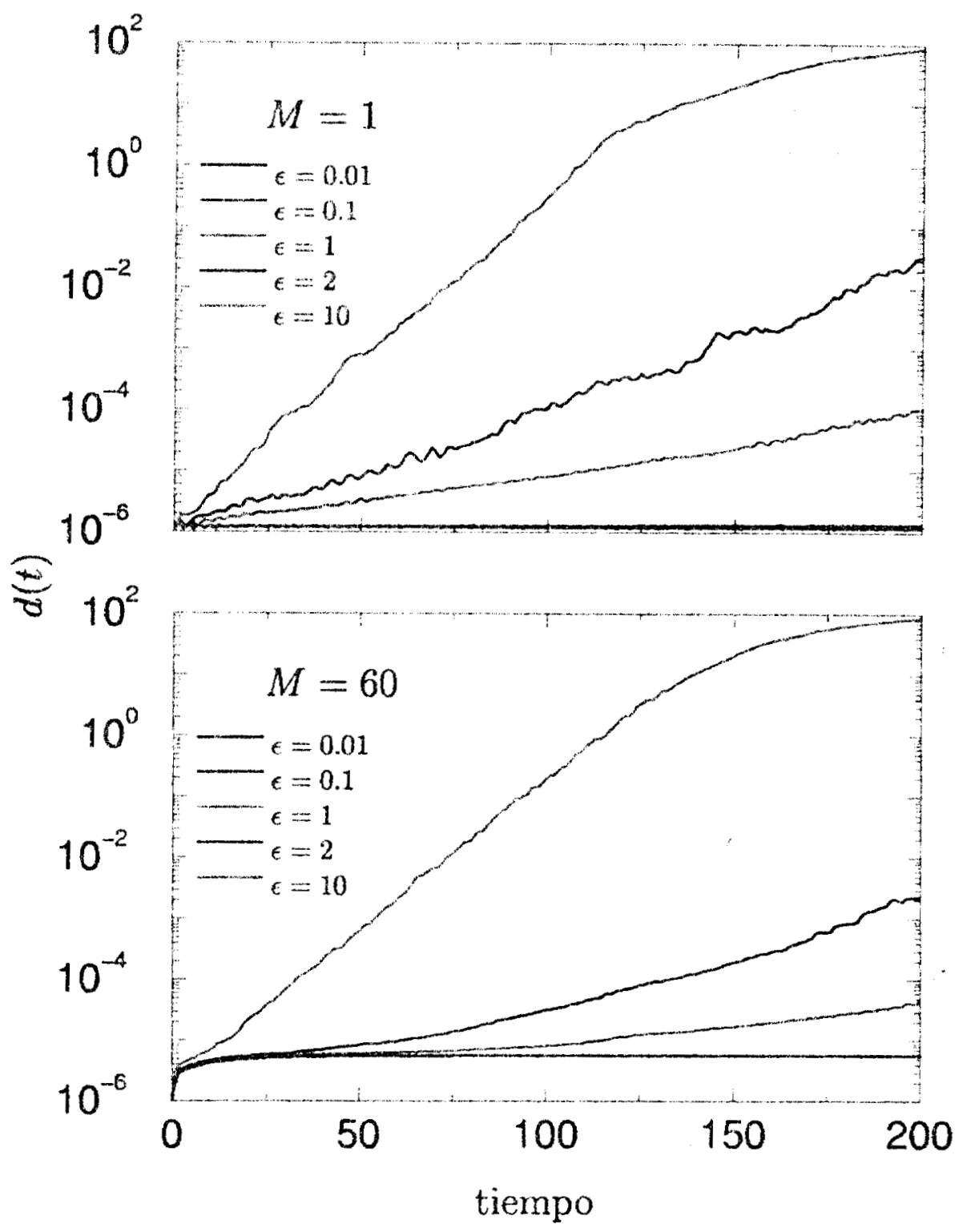

Figura 5.2: Distancia en el espacio fase entre pares de trayectorias correspondientes a diferentes condiciones iniciales determinadas por $\epsilon$ (densidad de energía total) $\operatorname{con} N=2000$.

al variar $N$ en aproximadamente dos órdenes de magnitud (de 2000 a 300000 ), por lo que podemos afirmar que estamos ante una característica genuinarnerte dinámica del modelo y que, según nuestra evidencia, es válida incluso en el límite termodinámico. Este hecho no es evidente a priori y concuerda cualitativamente 
con los resultados obteridos para el modelo FPU- $\beta$ por medio del máximo exponente de Lyapunov, tanto numéricamente [67] como analíticamente $[99,100]$. Podemos concluir entonces que los valores de $\epsilon$ ya reportados en la literatura, y que corresponden a $\epsilon \ll 1$ para un comportamiento regular y a $\epsilon \geq 1$ para un comportamiento caótico, son prácticamente independientes de la presencia del oscilador pesado.

\subsection{Equilibración del Sistema}

Hasta este momento hemos estudiado el comportamiento mecánico de nuestro modelo. El siguiente paso será estudiar su comportamiento estadístico, principalmente a través de la influencia del baño de osciladores livianos sobre el oscilador pesado. Como ya habíamos antes mencionado, las condiciones iniciales de nuestras simulaciones son compatibles con un cierto comportamiento dinámico, pero el estado que éstas definen no corresponde estrictamente al del equilibrio termodinámico, el cual esté́ definido por el principio de equipartición

$$
M\left\langle V_{0}^{2}\right\rangle=k_{B} T,
$$

donde $V_{0}(t)$ es la velocidad del osciladior pesado y $T$ es la temperatura del baño de osciladores livianos. Esta condición la hemos utilizado desde el primer capítulo para definir el régimen en el cual deben de hacerse todas las mediciones de las propiedades del oscilador pesado. Una primera característica de este principio que conviene resaltar es que es una relación escrita en términos de promedios estadísticos, que son invariantes ante una transformación canónica del tipo (4.2) de las variables microscópicas; por lo tanto, no tenemos una forma única de elegir la representación más adecuada de las mismas. Una posible elección correspondería escribir la relación (5.9) en términos de los modos normales del sistema, pero la transformación implicada complicaría inecesariamente nuestro tratamiento, ya que la elección (5.7) de las condiciones iniciales equivale a distribuir una cantidad aproximadamente igual de energía cinética entre todos los grados de libertad del sistema. Esta característica hace que la representación en términos de posiciones y momentos sea la más adecuada para nuestros propósitos. Ahora bien, debido a la presencia de los términos potenciales en el Hamiltoniano (5.4) la evolución temporal redistribuirá parte de esa energía hasta que, después de un cierto tiempo transitorio, la igualdad (5.9) se satisfaga con un grado razonable de precisión 
para todo tiempo posterior. A este proceso lo definimos como relajación hacia el equilibrio.

A continuación tenemos que determinar la forma de calcular el promedio $\langle\cdots\rangle$, así como la manera de obtener $T$ a partir de las variables dinámicas utilizando ese mismo promedio. Una posibilidad seria la de calcular $(\cdots)$ como un promedio sobre un ensamble en el cual cada elemento del mismo corresponde a una repetición del "experimento" con unas condiciones iniciales distintas para cada miembro del ensamble. Aunque es pasible estudiar el modelo FPU utilizando esta estrategia [116], la construcción de este tipo de ensamble es impráctica debido a la cantidad de recursos computacionales necesarios. La otra posibilidad, que es más natural debido a nuestro conocimiento completo de la dinámica microscópica, consiste en sustituir el promedio sobre un ensamble por un promedio temporal infinito $^{1}$; esto es, para una propiedad $f$ arbitraria tenemos que $\langle f\rangle=\lim _{t \rightarrow \infty}\langle f\rangle_{t}$, donde

$$
\langle f\rangle_{t}=\frac{1}{i} \int_{0}^{t} f\left(\left\{X_{i}\left(t^{\prime}\right), P_{i}\left(t^{\prime}\right)\right\}\right) d t^{\prime}
$$

es un promedio sobre un intervalo finito de tiempo $t$. Si el intervalo de tiempo es lo suficientemente grande, basta con tomar una sola trayectoria en el espacio fase (un solo "experimento") para calcular el promedio deseado con un grado de aproximación razonable. Ahora, para el oscilador pesado la propiedad de la cual tenemos que conocer su evolución temporal ticne que ser su energía cinética

$$
K_{P H}=M V_{0}^{2} \text {. }
$$

En cuanto al baño de osciladores livianos, la propiedad dinámica equivalente corresponde a la energía cinética por grado de libertad, definida como

$$
K_{B T}=\frac{1}{N} \sum_{i=1}^{N} V_{i}^{2}(t) .
$$

A partir de esta última propiedad podemos definir la temperatura cinética como $T_{c i n}=\lim _{t \rightarrow \infty}\left\langle K_{B T}\right\rangle_{t}$ en unidades de $k_{t}$, lo que implica que debemos tomar $k_{B}=1$. Entonces el principio de equipartición nos dice que, asintóticamente, debe de cumplirse la siguiente igualdad

$$
\lim _{t \rightarrow \text { grande }}\left\langle K_{P B}\right\rangle_{t}=T_{c i n}
$$

\footnotetext{
${ }^{1}$ La equivalencia entre promedios sobre el ensamble y promedios temporales es uno de los aspectos del problema ergódico ya mencionado en el capítulo anterior. Puesto que este problema no es relevante para el presente trabajo, supondremos la validez de la equivalencia entre estos promedios.
} 
Hemos hecho la sustitución " $t \rightarrow$ grande" por " $t \rightarrow \infty$ " porque este último es un límite meramente forraal; los tiempos de cómputo siempre son finitos (aunque pueden ser muy grandes, como tendremos ocasión de ver más adelante). Además, en los tiempos de cómputo a nuestro alcance debe de ser posible observar que la igualdad (5.13) se satisfaga de manera muy aproximada, ya que, de lo contrario, nuestra metodología sería inutil para estudiar el problema que nos interesa.

Antes de continuar es conveniente hacer algunas últimas observaciones sobre el principio de equipartición. La idea física que subyace a este principio es que el efecto del oscilador pesado sobre el baño de asciladores debe de ser despreciable; esto es, las propiedades estadísticas del baño no deben de verse mayormente afectadas por la presencia del defecto en la cadena. Ya hemos visto en la sección anterior que, para algunas propiedades dinámicas, el efecto parece ser efectivamente despreciable, lo que ofrece una primera evidencia, aunque indirecta, en este sentido. Por otra parte, el efecto del baño sobre el oscilador pesado debe de ser de tal magnitud que este ultimo alcance el estado de equilibrio termodinámico con el primero, independientemente de la energía cinética que inicialmente posea. Por último, la validez de (5.13) es de caracter puramente estadístico; esto es, su validez depende del número $N$ de osciladores livianos. El valor específico de $N$ tiene una importancia marginal desde el punto de vista dinámico, por lo que, hasta este momento, ese valor ha sido en gran medida arbitrario. Nuestro siguiente objetivo será obtener una estimación de $N$ tal que permita satisfacer el principio de equipartición en un tiempo al alcance de los recursos de cómputo disponibles. No es posible conocer este número de antemano debido a la falta de estimaciones analíticas para este problema. Sólo podemos anticipar que debe de ser muy grande.

\subsubsection{Primeros Resultados}

Nuestras primeras simulaciones empezaron con las condiciones iniciales definidas por (5.7) y un número de osciladores livianos de $N=2000$, elegido en gran medida porque nos permitió hacer comprobaciones muy rápidas del comportamiento del sistema. Los promedios $\left\langle K_{P B}\right\rangle_{t}$ y $\left\langle K_{B T}\right\rangle_{t}$ fueron calculados utilizando (5.10). Para los casos $\epsilon=0.01$ (régimen armónico) y $\epsilon=10$ (régimen caótico) los resultados se muestran en la figura 5.3 .

Asintóticamente es claro que $\left\langle K_{H T}\right\rangle_{t}$ tiene el comportamiento esperado. De hecho, el valor de este promedio se estabiliza para $t \simeq 1000$, por lo que la iden- 

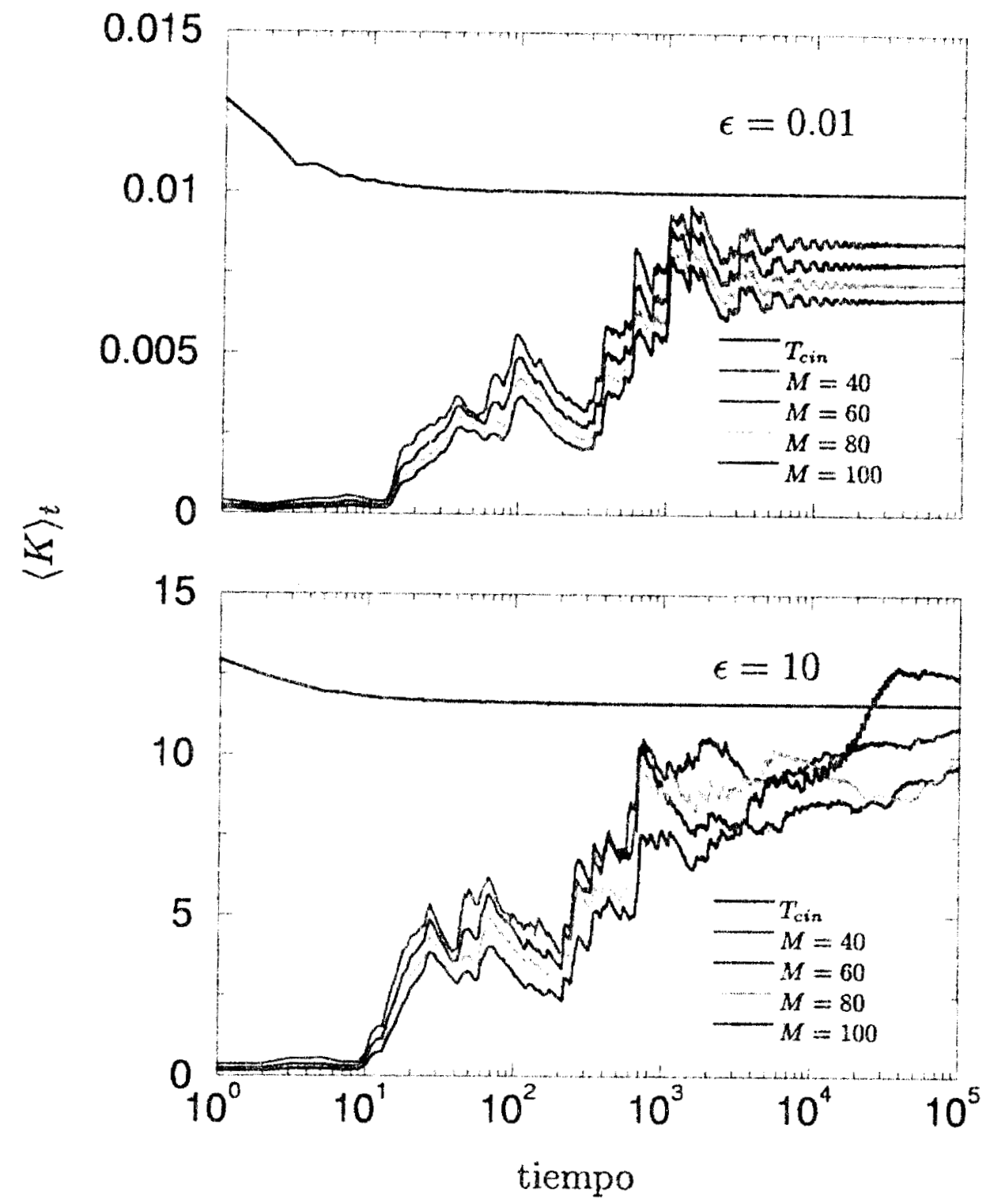

Figura 5.3: $T_{\text {cin }} y\left(K_{p_{B}}\right)_{t}$ vs tiempo para los distintos valores $M$ utilizados. Los valores de la densidad de energía corresponden a los regímenes armónico $(\epsilon=0.01)$ y caótico $(\epsilon=10) . N=2000$.

tificación de $T_{c i n}$ con el límite asintótico de $\left\langle K_{B T}\right\rangle_{t}$ está plenamente justificada. Podemos afirmar entonces que el baño de osciladores livianos, tomado de manera aislada, se comporta efectivamente como un baño termodinámico. Sin embargo, la situación resulta ser totalmente diferente para $\left\langle K_{p g}\right\rangle_{t}$ : de la figura resulta 
evidente, en particular para el caso $\epsilon=0.01$, que el sistema completo evoluciona asintóticamente hacia un estado metaestable que no corresponde al estado. de equilibrio termodinámico definido por el principio de equipartición (5.13). La situación para el caso $\epsilon=10$ es cualitativamente similar, aunque cabe hacer notar que, para el intervalo de tiempo mostrado, parece ya existir una tendencia hacia el equilibrio, sobre todo para $M=100$. En principio este comportamiento sería de esperarse, puesto que la presencia del potencial cuártico en (5.4) facilita el intercambio de energía entre los distintos grados de libertad del sistema. Sin embargo, esta tendencia resulta ser sólo aparente; si continuamos el cálculo para un intervalo de tiempo ciliez veces más grande que el mostrado en la figura 5.3 no encontramos evidencia de un eventual relajamiento hacia el estado de equilibrio para ninguno de los valcres de $M$ estudiados.

¿Cómo podemos entender estos resultados? $\mathrm{El}$ caso $\epsilon=0.01$ de la figura 5.3 parece sustentar el enfoque de Khinchin [76] según el cual la ergodicidad de los observables físicos relevantes es una consecuencia del gran número de grados de libertad involucrados más que del comportamiento caótico del sistema. Una muestra de ello es que el efecto de la dinámica en la equilibración es muy débil, puesto que el estado metaestable se alcanza muy rápidamente sin ninguna evidencia posterior de una eventual relajación al equilibrio. Además, el efecto de la masa $M$ es muy regular, disminuyendo el valor asintótico de $\left\langle K_{p_{B}}\right\rangle_{t}$ conforme aumenta el valor de $M$. Sin embargo, la situación para $\epsilon=10$ es dramáticamente distinta. Se observa claramente la infuencia del potencial anarmónico en la termalización del sistema, acelerándola y volviendo el efecto de $M$ sobre la misma menos regular. Estos resultados son una clara confirmación de que el comportamiento estadístico de un sistema dinámico resulta de un efecto combinado, tanto del número de grados de libertad como de la dinámica intrínseca del sistema. Adicionalmente, en nuestro problema particular el efecto de la masa del oscilador pesado es el de crear una separación de escalas de tiempo muy pronunciada entre los distintos grados de libertad del sistema (este efecto es característico del movimiento Browniano y tendremos ocasión de volver a estudiarlo en el capítulo siguiente). Mientras que el número $N=2000$ de osciladores livianos es suficiente para llevar a un estado de equilibrio termodinámico a cualquiera de ellos con respecto a los demás, resulta insuficience para termalizar al oscilador pesado en la misma escala de tiempo. Esta termalización tiene que conseguirse a través de una transferencia recíproca de energía entre el baño térmico y el oscilador pesado. Pero parece claro que, para $N=2000$, esto no sería posible sin que el baño de osciladores livianos 
pierda sus propiedades estadísticas (esto es, que deje de comportarse como baño térmico). Para el caso $\epsilon=10$ la interacción debida al potencial cuártico está claramente facilitando la transferencia de energía ya antes aludida, pero la eficacia de este mecanismo resulta muy pobre, por lo que el oscilador pesado y el baño de osciladores livianos se comportan como sistemas independientes. Concluimos entonces que el único mecanismo disponible para conseguir la adecuada termalización del sistema consiste en aumentar el número de osciladores livianos de tal manera que (5.13) se satisfaga dentro de unos límites aceptables, ya que deseamos conservar, tanto la unidimensionalidad como el Hamiltoniano (5.4).

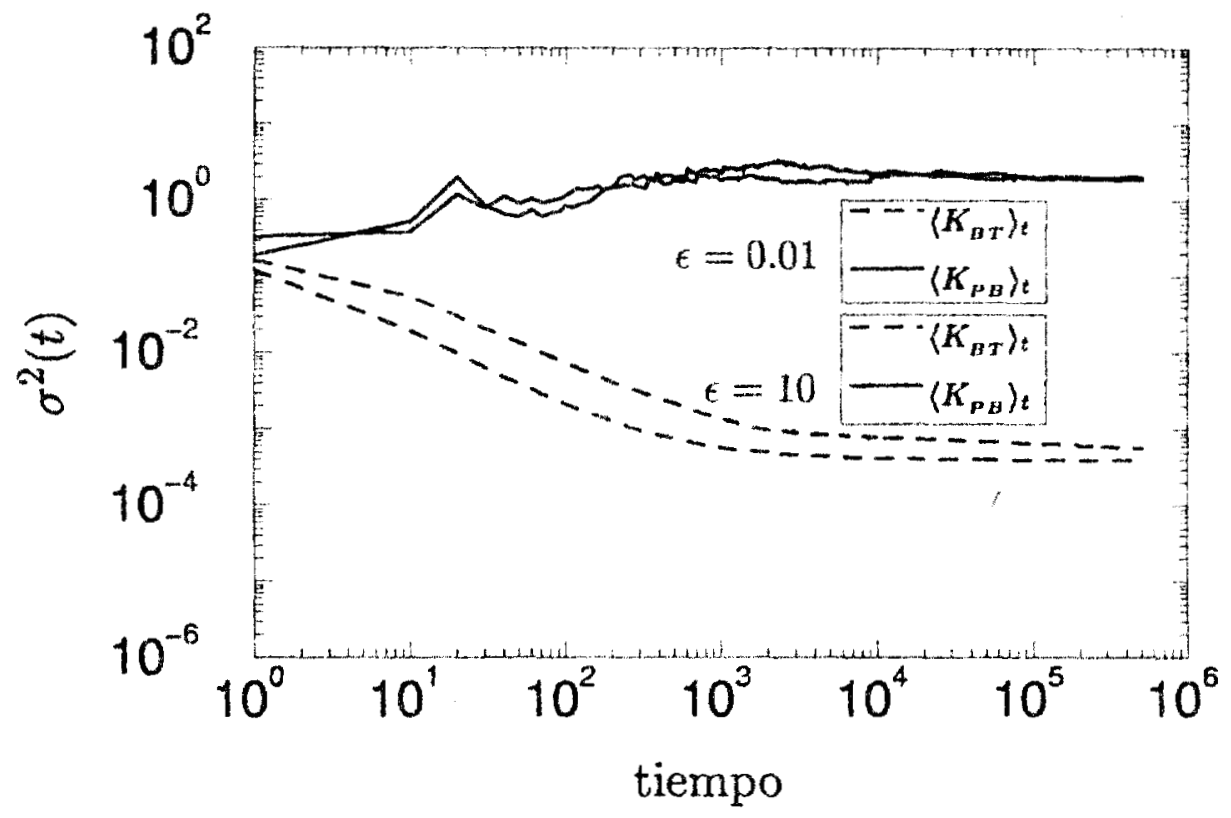

Figura 5.4: Fluctuación relativa de $\left(K_{B T}\right\rangle_{t}$ (curvas inferiores) y $\left\langle K_{p_{B}}\right\rangle_{t}$ (curvas superiores) para $\epsilon=0.01$ y $\epsilon=10 \mathrm{con} M=100$ y $N=2000$ en ambos casos.

Podernos ampliar nuestra comprensión de esta fenomenología estudiando no solo los promedios $\left\langle K_{B T}\right\rangle_{t}$ y $\left\langle K_{P B}\right\rangle_{t}$, sino también las correspondientes fluctuaciones relativas calculadas a través de

$$
\sigma_{f}^{2}=\frac{\left\langle f^{2}\right\rangle_{t}-\langle f\rangle_{t}^{2}}{\langle f\rangle_{t}^{2}}
$$

donde $f$ puede ser $K_{B T} \circ K_{P B}$. Los resultados para $\epsilon=0.01$ y $\epsilon=10$ cuando $M=100$ se muestran en la figura 5.4. Es claro que, aunque el valor asintótico 
$T_{c i n}$ del promedio $\left\langle K_{B T}\right\rangle_{t}$ se alcanza en un tiempo relativamente corto, las fluctuaciones de esta cantidad empiezan a estabilizarse alrededor de un valor constante $\sim O(1 / N)$ en una escala de tiempo más grande, $t_{e} \simeq 2000$ para el caso mostrado. Cabe mencionar que hay diferencias entre los casos armónico y caótico, ya que, mientras que en el segundo es evidente que el valor asintótico de las fluctuaciones ya es constante cuando $t \simeq t_{e}$, en el primero todavía se observa una tendencia decreciente para $t>t_{e}$, aunque con un ritmo mucho menor y dentro de los límites estadísticos adecuados. 'Toda esta evidencia es congruente con un comportamiento mecanico-estadístico normal para el baño de osciladores. Sin embargo, las fluctuaciones relativas de $\left\langle K_{P_{B}}\right\rangle_{t}$ tienen una magnitud mucho mayor y no muestran una dependencia con respecto a $N$ en ninguno de los dos casos, armónico y cático. Para los otros valores de $M$ estudiados los resultados son virtualmente idénticos, ya que la manera de calcular $\sigma^{2}$ excluye toda dependencia con respecto a $M$. Hemos confirmado así las observaciones hechas en el párrafo anterior referidas al valor que debe de tener $N$ para obtener una adecuada termalización del sistema, tanto para el régimen armónico como para el caótico, pues el comportamiento es bastante similar en ambos casos.

Pero, a pesar de todos los resultados ya presentados, falta hacer una última comprobación: esta consiste en averiguar si la fenomenología anteriormente estudiada corresponde a una característica global del sistema, dependiente sólo del valor de $M$ y del número de osciladores, o bien es una característica local dependiente de las condiciones iniciales utilizadas (aunque estas sean del caracter más general posible). Para responder a esta interrogante hemos considerado el siguiente conjunto alternativo de condiciones iniciales

$$
\begin{aligned}
P_{i}(0) & =0 \\
X_{i}(0) & =\sum_{n=1}^{N / 2}\left(\frac{A}{n}\right) a(n) U_{i}(n),
\end{aligned}
$$

donde

$$
a(n)=\sin \left(\frac{\pi n}{N}\right) \cos \psi_{n},
$$

siendo $\psi_{n}$ una variable aleatoria uniformemente distribuida en el intervalo $0-2 \pi$, mientras que

$$
U_{i}(n)=\frac{[\cos (2 \pi i n / N)-Q \tan (\pi n / N) \sin (2 \pi i n / N)]}{\left[1+Q^{2} \tan ^{2}(\pi n / N)\right]^{1 / 2}} i=0, \ldots, N
$$




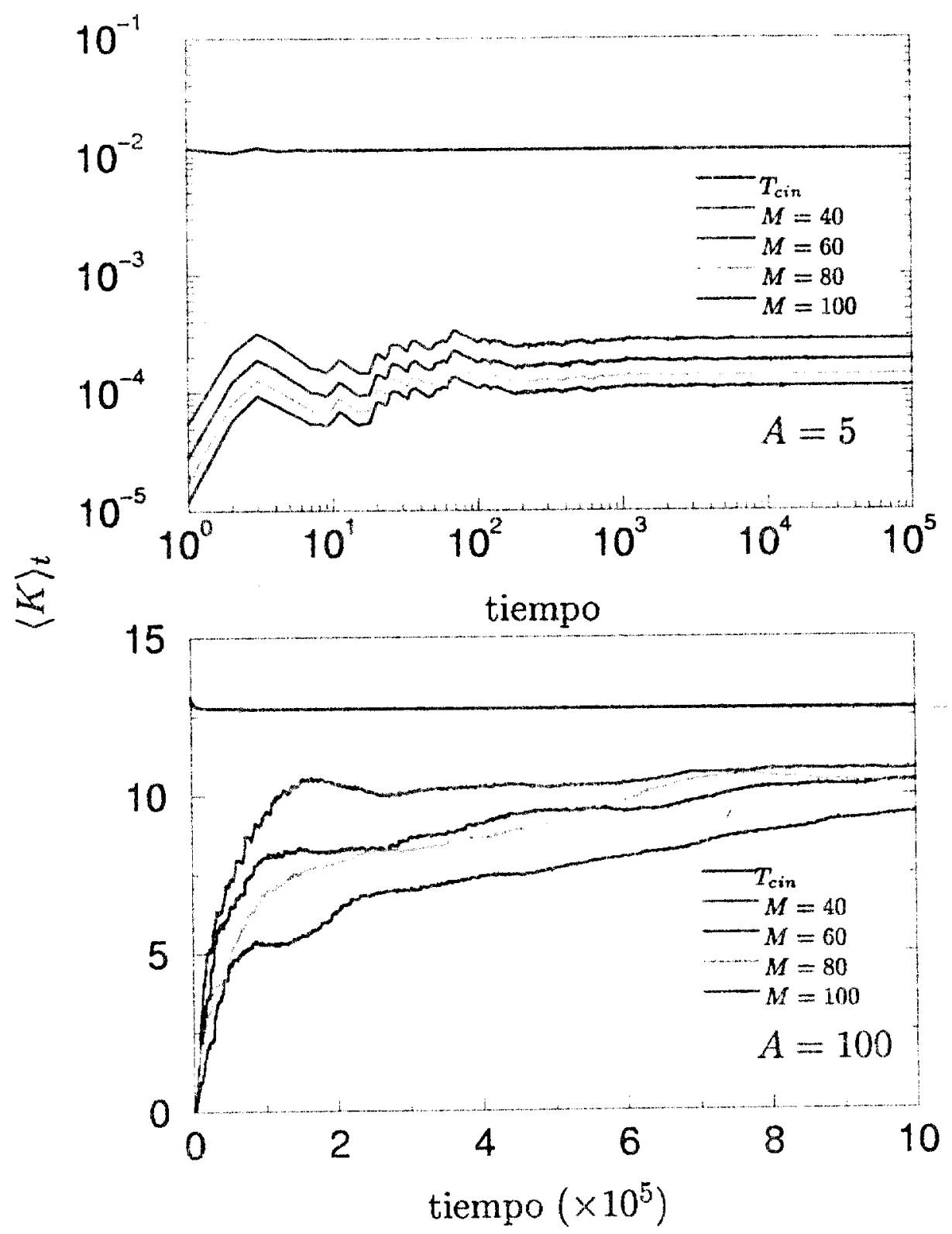

Figura 5.5: $T_{c i n}$ y $\left\langle K_{p B}\right\rangle_{t}$ vs tiempo para los mismos valores de $M$ reportados en la figura 5.3, pero con unas condiciones iniciale; dadas por (5.15)-(5.18). Para $A=5, \epsilon \approx 0.01137 \mathrm{y}$, para $A=100$, $\epsilon \approx 10.8432$. Obsérvese la escala $\log$ - $\log$ para $A=5$.

son las amplitudes reducidas (A.27) tomando la aproximación $\phi_{k} \approx 2 \pi n / N$. Puesto que, en equilibrio, los momentos $\left\{P_{i}\right\}$ deben de ser variables Gaussianas 
con media cero, el hacer $P_{i}(0)=0$ equivale a tomar el valor más probable para las mismas. La componente aleatoria en $\left\{X_{i}(0)\right\}$ está realacionada con la imposibilidad de preparar cualquier sistema físico en un estado inicial perfectamente ordenado: para una temperatura distinta de cero es inevitable un cierto grado de aleatoriedad en las condiciones iniciales. Sin embargo, como las posiciones iniciales están dadas en términos del conjunto completo $\{\mathrm{U}(n)\}$, tenemos que las condiciones iniciales (5.15)-(5.18) corresponden a una subvariedad (toroides KAM) ligeramente perturbada del espacio fase, lo que las hace cualitativamente diferentes a las condiciones (5.7) utilizadas hasta este momento.

En la figura 5.5 se muestra el resultado para $A=5(\epsilon \approx 0.01137)$ y $A=100$ $(\epsilon \approx 10.8432)$ con los mismos valores de $M$ que en la figura 5.3. El comportamiento de $\left\langle K_{B T}\right\rangle_{t}$ es muy sirnilar al caso correspondiente a las condiciones iniciales aleatorias (5.7) e incluso el valor asintótico $T_{c i n}$ se alcanza en una escala de tiempo mucho más pequeña. Por otra parte, el comportamiento asintótico de $\left\langle K_{p_{B}}\right\rangle_{t}$ es muy similar al ya anteriormente encontrado, resultando mucho más pronunciado en el régimen armónico $(\epsilon=0.01)$. Estos resultados muestran claramente que, al empezar el cálculo en un punto de una región ordenada del espacio fase, resulta todavía más difícil obtener un comportamiento estadístico adecuado. Ahora bien, como la fenomenología obtenida sólo difiere cuantitativamente de aquella obtenida con las condiciones iniciales (5.7), podemos concluir que efectivarnente estamos ante una característica global del sistema y que, al parecer, sólo podrá ser modificada aumentando el número de osciladores livianos, como ya lo habíamos planteado.

\subsubsection{Resultados en el Límite Termodinámico}

La situación cambia dramáticamente cuando se incrementa el número $N$ de osciladores del baño térmico. En la figura 5.6 se muestra una simulación con los mismos parámetros utilizados en la figura 5.3, pero ahora utilizando un baño de 300000 osciladores livianos. El criterio utilizado para determinar el valor de $N$ fue el de poder obtener, en la escala de tiempo mostrada, un valor asintótico de $\left\langle K_{P B}\right\rangle_{t}$ que satisfaciera el principio de equipartición (5.13) lo mejor posible para el caso $\epsilon=0.01$. Este criterio fue establecido con base a que estamos estudiando los diversos casos caciticos tomando como referencia el comportamiento del correspondiente caso armónico, por lo que es indispensable conocer en detalle la evolución temporal de los promedios asociados a este caso particular. 


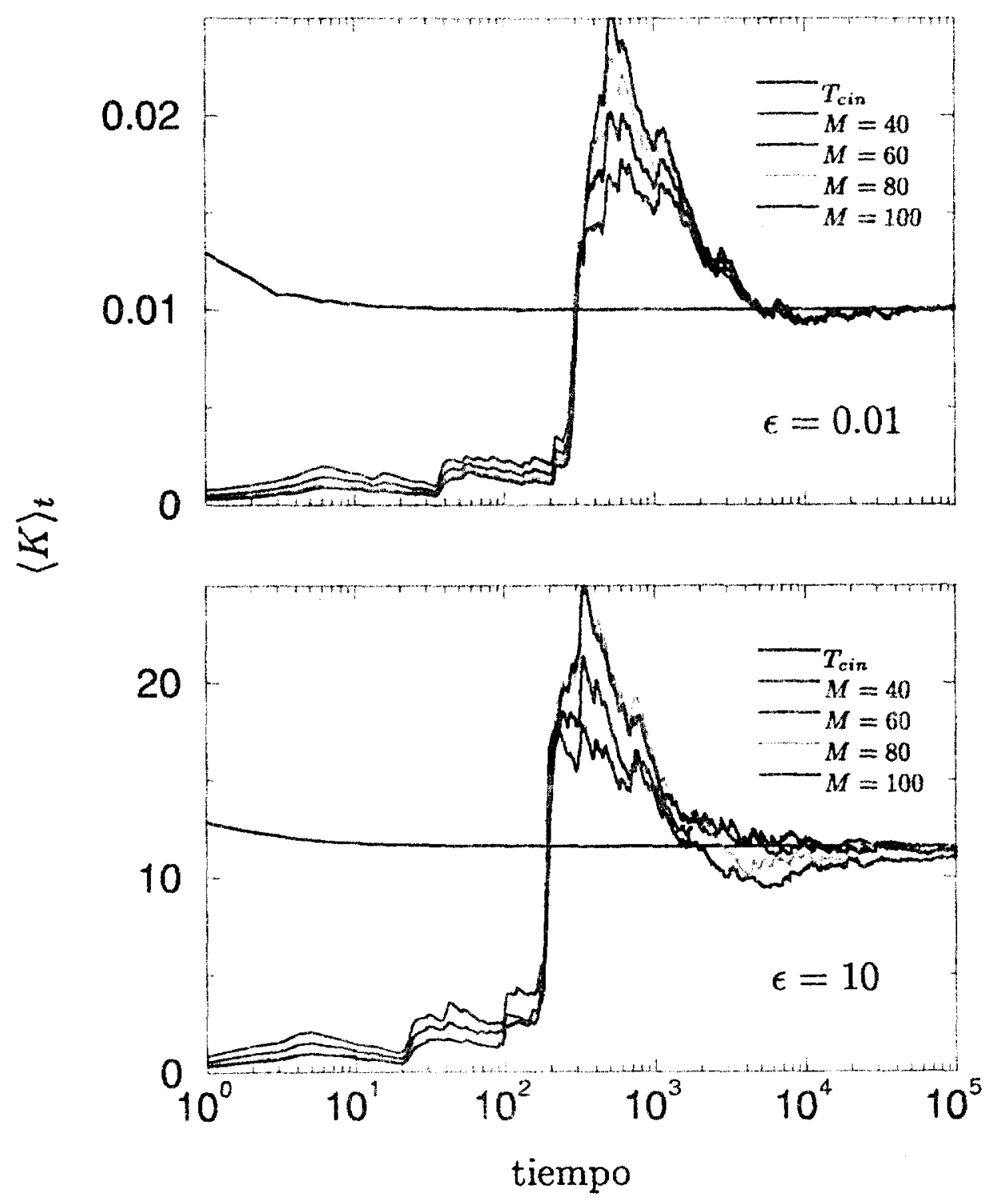

Figura 5.6: Mismos parámetros que en la figura 5.3, pero ahora $\operatorname{con} N=300000$ osciladores livianos.

En la escala de las figuras se observa que, para $\epsilon=0.01$, el sistema ha relajado hacia un estado muy cercano al equilibrio termodinámico, como se esperaba. Puede observarse que en este régimen existe una cierta universalidad en el cornportamiento del promedio $\left\langle K_{P_{B}}\right\rangle_{t}$ para las distintas masas consideradas, ya que todas las curvas tienden asintóticamente de la misma manera al valor de $T_{c i n}$. 
Además, es claramente visible que se desarrolla un "pico" en cada una de las curvas correspondientes a $\left\langle K_{\boldsymbol{P}_{\boldsymbol{B}}}\right\rangle_{t}$ antes de que el sistema finalmente relaje hacia el equilibrio, siendo esta una característica que se vuelve más pronunciada conforme aumenta el tarnaño del baño, como puede verse al comparar con la figura 5.3, y que se presenta en un tiempo más corto para el caso caótico $(t \simeq 325)$ que en el caso armónico $(t \simeq 532)$. Este fenómeno es semejante al del "deslizamiento" de las condiciones iniciales en una dinámica reducida de sistemas acoplados a baños térmicos [117] y también al de la evolución temporal de la función $H$ de Boltzmann para un fluido de Lennard-Jones [118]; en particular, en este último caso las condiciones iniciales son idénticas a las que hemos estado utilizando. Para $\epsilon=10$ obtenemos, sin embargo, un resultado totalmente inesperado: el valor asintótico de equilibrio para $\left\langle K_{p_{B}}\right\rangle_{t}$ todavía no ha sido alcanzado. Este comportamiento no se entiende del todo en vista de los resultados obtenidos para $N=2000 \mathrm{y}$ de lo que esperaríamos de un Hamiltoniano con un término cuártico como (5.4), ya que en este tipo de sistemas es razonable esperar una rápida redistribución de la energía entre todos los grados de libertad mucho más rápida que en el correspondiente caso de un potencial puramente cuadrático. En cuanto al origen de este comportarniento, en este momento sólo podemos hacer algunas suposiciones. Observemos en primer lugar que, tomando corno referencia el caso armónico, el número de osciladores ya es lo suficientemente grande como para considerar que, dentro de los límites impuestos por una metodología de caracter numérico, estamos trabajando muy aproximadamente en el límite termodinámico $(N \rightarrow \infty)$. Esto implica que hemos podido eliminar en gran medida los efectos sobre la equilibración del sistema debidos al número de osciladores y que eran muy evidentes cuando tomamos $N=2000$. Ahora, como un primer intento de cuantificar el tiempo de equilibración del sistema estudiaremos las fluctuaciones relativas de $K_{B T}$ y $K_{P B}$, las cuales se muestran para el caso particular de $M=100$ en la figura 5.7. Para el caso de $T_{c i n}$ las fluctuaciones relativas empiezan a estabilizarse alrededor de un valor constante $\sim O(1 / N)$ en una escala de tiempo $t_{\mathrm{e}} \simeq 5 \times 10^{5}$, mucho más grande que en el caso análogo con $N=2000$. Sin embargo, ésta es la única dependencia en $N$ que puede observarse, ya que las fluctuaciones relativas de $\left\langle K_{P B}\right\rangle_{t}$, tanto para $\epsilon=0.01$ como para $\epsilon=10$, tienen un comportamiento muy parecido al de sus contrapartes para el caso de $N=2000$. Esto nos muestra que las propiedades estadísticas de las fluctuaciones relativas de $\left\langle K_{P B}\right\rangle_{t}$ permanecen en gran medida inalteradas a medida que $N$ se incrementa en varios órdenes de magnitud, por lo que eis muy difícil inferir, a partir de su comportamiento, si el 
sistema ha alcanzado el estado de equilibrio termodinámico definido por (5.13). A pesar de esto, el comportamiento asintótico de $\left\langle K_{p R}\right\rangle_{t}$ parece ser mucho rás regular que para $N=2000$ y el de sus fluctuaciones relativas no resultan mayormente afectadas por el valor de $M$. Estas observaciones hacen plausible considerar que $t_{e}$ puede tomarse, en una primera aproximación, como un indicador efectivo del grado de equipartición que ha alcanzado el sistema.

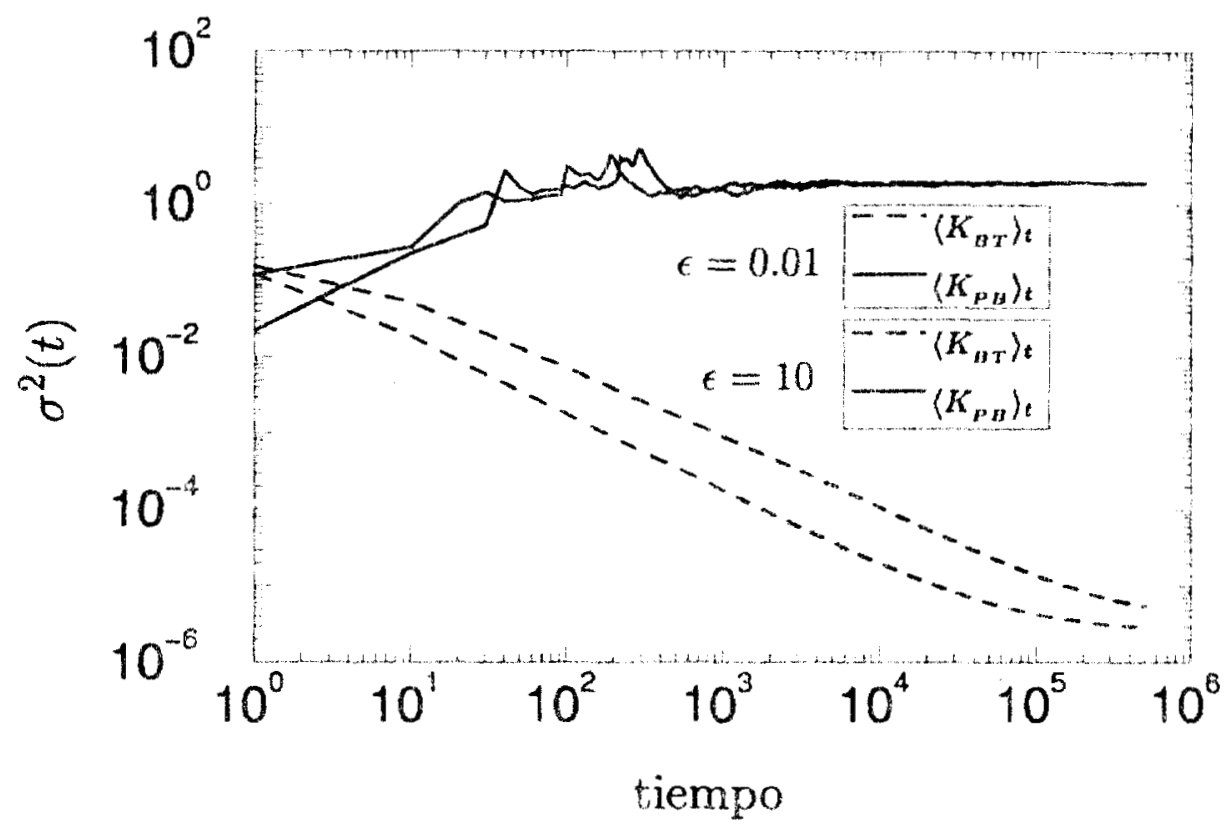

Figura 5.7: Mismos parámetros que en la figura 5.4, pero para $N=300000$ osciladores livianos.

Ahora bien, si deseamos investigar el ritmo de termalización con más detalle tenemos que utilizar un parámetro diferente, el cual debe poder cuantificar, de una manera sencilla, qué tan alejado se encuentra el valor asintótico de $\left\langle K_{P B}\right\rangle_{t}$ del valor de $T_{c i n}$. De esta manera será posible investigar el efecto, evidente en la figura 5.6, del valor de $M$ en la relajación hacia el equilibrio. Proponemos entonces la función

$$
\mathcal{D}_{K}(t)=\frac{1}{T_{c i n}}\left|\left\langle K_{P B}\right\rangle_{t}-T_{c i n}\right|
$$

la cual proporciona una estimación de la diferencia relativa entre $\left\langle K_{P_{B}}\right\rangle_{t}$ y el valor hacia el cual debe de tender asintóticamente, $T_{c i n}$. De hecho, en ese régimen temporal $\mathcal{D}_{K}(t)$ debe de satisfacer la propiedad

$$
\lim _{t \rightarrow \text { grande }} \mathcal{D}_{K}(t)=0
$$


Consideramos que una diferencia porcentual de $1 \%\left(\mathcal{D}_{K}(t) \leq 1\right)$ garantiza que el equilibrio termodinámico ya ha sido alcanzado y que puede entonces hacerse el estudio del movimiento Browniano, como mostraremos en el capítulo siguiente. Cabe mencionar que nuestra función $\mathcal{D}_{\kappa}(t)$ tiene cierto parecido con el estimador introducido por Thirumalai y Mountain para estudiar la equipartición de energía en el modelo FPU, el cual consiste esencialmente en una norma sobre el espacio de un cierto observable obtenida a partir de dos trayectorias diferentes [94]. En nuestro caso tenemos dos propiedades distintas cuyos promedios temporales sirven para construir una norma que está evaluada sobre una misma trayectoria en el espacio fase.

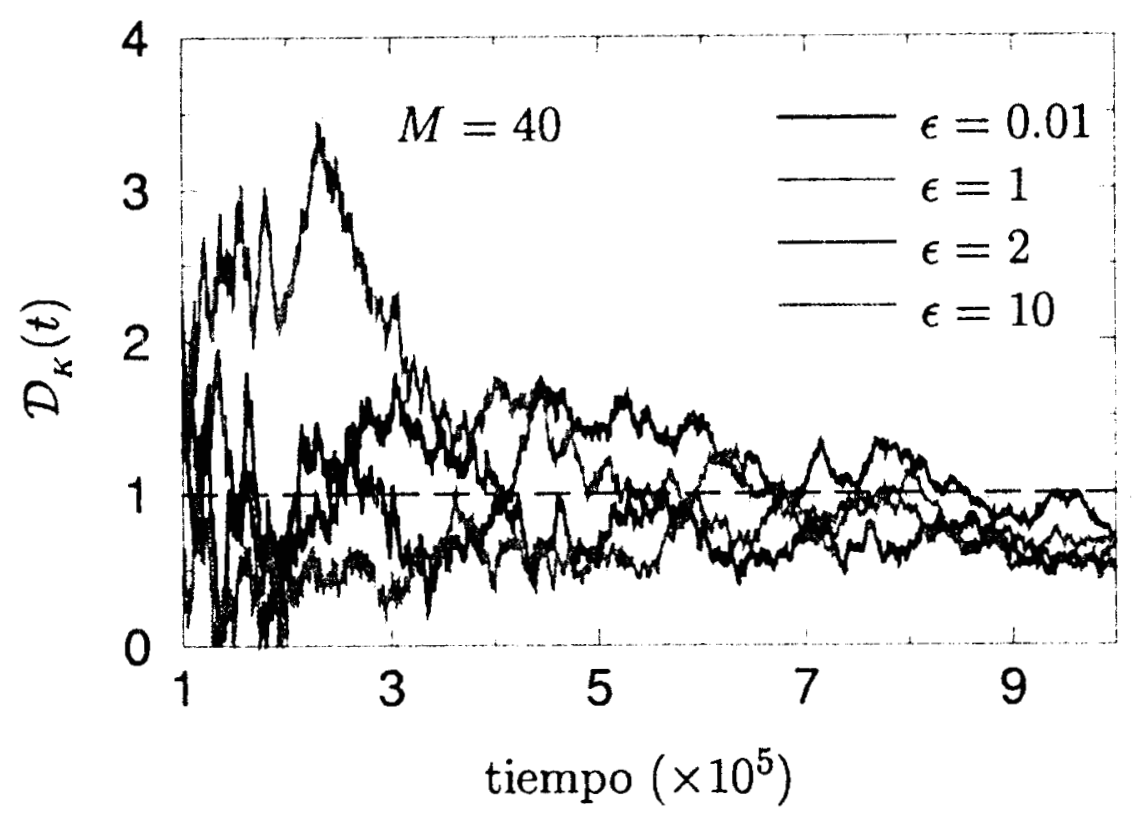

Figura 5.8: Diferencia relativa para $M=40$ y $N=300000$.

El resultado para $M^{\prime}=40$ y algunos valores representativos de $\epsilon$ puede apreciarse en la figura 5.8. Resulta claro que, asintóticamente, $\mathcal{D}_{K}(t)<1$ para todos los casos considerados. Sin embargo, la escala temporal $t \approx 9 \times 10^{5}$ en la que se presenta este comportamiento es muy grande, más grande de hecho que la escala de tiempo $t_{e}$ calculada a partir de las fluctuaciones relativas de $K_{P_{B}}$, como ya lo mencionamos con anterioridad. Recordemos, sin embargo, que la estimación de $t_{e}$ se realizó tomando en cuenta únicamente el valor $M=100$, por lo que es de esperar que el estudio del comportamiento asintótico de $\mathcal{D}_{\kappa}(t)$ para diversos valores 
de $M$ proporcione información mucho más detallada que aquella disponible hasta este momento. Observamos, además, que los valores más grandes de la diferencia porcentual se presentan para $t<3 \times 10^{5}$, la cual es una escala de tiempo en la que ya habíamos considerado que el sistema todavía no alcanza el estado de equilibrio ternodinámico. Por lo tanto, la información que tenemos hasta el momento es congruente con el criterio de equilibración ya establecido a través de $t_{\mathrm{e}}$. El comportamiento de $\mathcal{D}_{K}(t)$ para el caso $M=60$ es muy similar, como puede observarse en la figura 5.9. La diferencia más notoria con respecto al caso anterior es la rapidez con la que el comportamiento asintótico de la diferencia porcentual se presenta. Para $t>2 \times 10^{5}$ tenemos que $\mathcal{D}_{K}(t) \ll 1$ en todos los casos anarmónicos considerados $(\epsilon \geq 1)$. Por otra parte, $\mathcal{D}_{K}(t) \approx 1$ para el caso armónico $(\epsilon=0.01)$ en la misma escala de tiempo, como se muestra. Por lo demás, la fenomenología es muy similar al caso $M=40$.

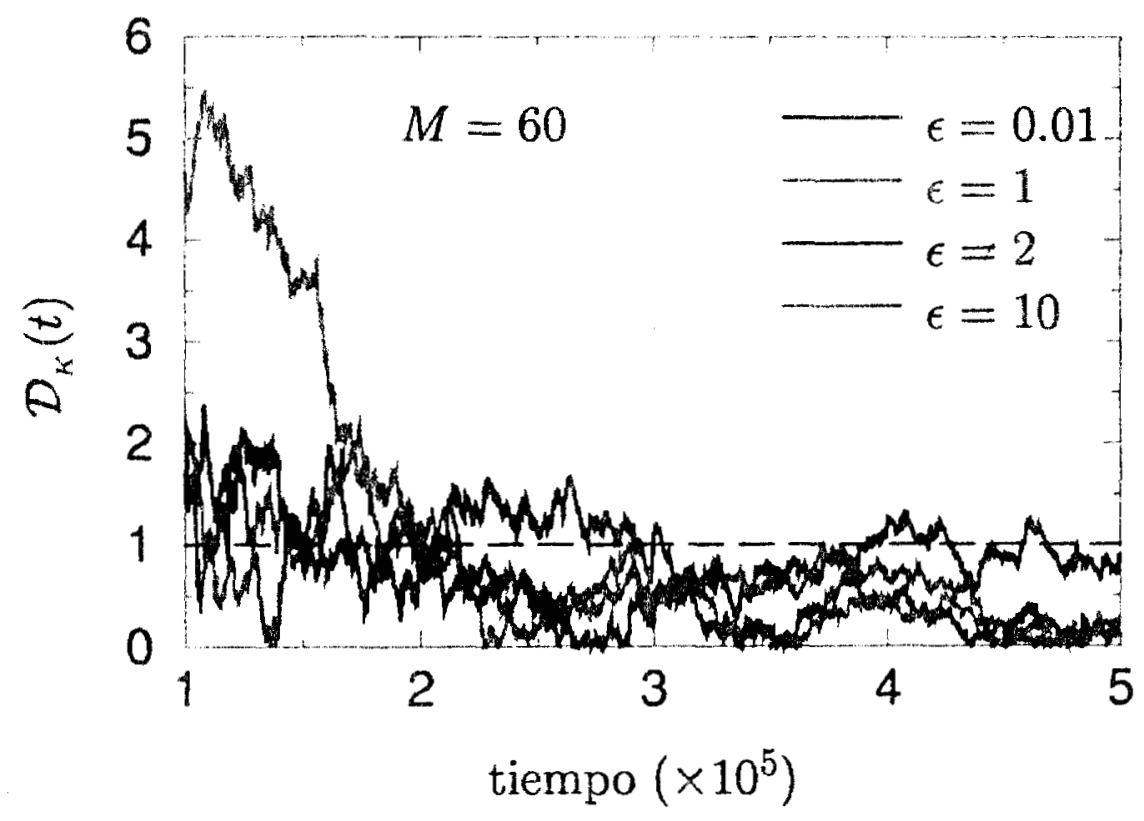

Figura 5.9: Diferencia relativa: $M=60$ y $N=300000$.

Pero este panorama cambia drásticamente para los casos $M=80$ y 100 mostrados en las figuras 5.10 y 5.11 , respectivamente. En particular, asintóticamente tenernos que $\mathcal{D}_{K}(t) \approx 1$ para $\epsilon=0.01$ en ambos casos; este comportamiento es muy semejante al caso análogo para $M=60$. Para $\epsilon=10$ tenemos que $\mathcal{D}_{K}(t) \ll 1$, siendo este comportamiento taribién muy semejante al caso análogo con $M=60$; 


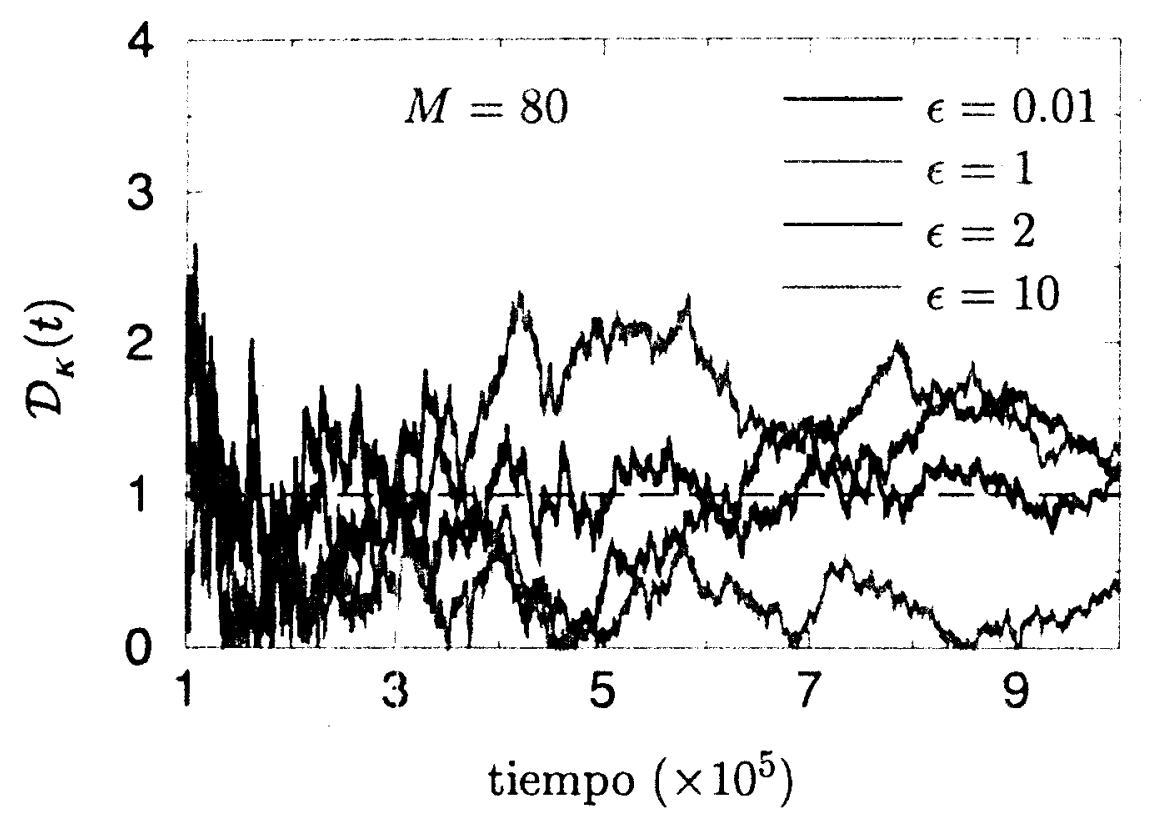

Figura 5..10: Diferencia relativa: $M=80$ y $N=300000$.

en particular, es el único caso anarmónico para el cual se cumple estrictamente que $\mathcal{D}_{K}(t)<1$ en el régimen temporal mostrado. Por lo tanto, podemos afirmar que la diferencia porcentual, para $\epsilon=0.01$ y 10 , tiene un comportamiento muy parecido en los casos $M=80$ y 100 . A continuación observamos que $\mathcal{D}_{K}(t)>1$ para $\epsilon=1$ y 2 en ambos casos considerados $(M=80$ y $M=100)$. Este comportamiento es muy diferente a aquel ya observado para $\epsilon=10$. Con la evidencia hasta el momento disponible no es fácil dar una explicación a esta fenomenología, aunque podría interpretarse como la manifestación de un cambio cualitativo de la dinámica del sistema cuando $\epsilon=1$ y 2 . Esta posibilidad será explorada de una manera totalmente diferente en el capítulo 6.

Teniendo en cuenta toda la información disponible sobre $\mathcal{D}_{K}(t)$ para los casos correspondientes a $M=40,60,80$ y 100 podemos establecer algunas conclusiones. Aunque sólo para $t \approx 9 \times 10^{5}$ el comportamiento de $\mathcal{D}_{K}(t)$ es lo suficientemente regular en todos los cascs considerados, también es claro que, para $t \approx 5 \times 10^{5}$, la diferencia porcentual $\mathcal{D}_{K}(t)$ ya no toma valores muy diferentes a 1 , por lo que podemos considerar que la información proporcionada por el comportamiento asintótico de $\mathcal{D}_{K}(t)$ es congruente con el valor de $t_{e}$ obtenido a partir de las fluctuaciones relativas de $K_{P B}$. La única excepción parece ser el caso $\epsilon=1$ para 
$M=80$, ya que $\mathcal{D}_{\kappa}(t)$ presenta un decaimiento apreciable cuando $t>5 \times 10^{5}$. En este momento no podemos decir si este resuitado tendrá consecuencias; sólo podremos averiguarlo cuando estudiemos el comportamiento del cscilador pesado en equilibrio termodinámico en el capítulo siguiente. Por último, podemos decir que, en vista de los resultados para $N=300000$ osciladores, hemos obtenido con un grado razonable de precisión el estado de equilibrio en todos los casos. Sin embargo, falta un estudio detallado de la dependencia de $t_{e}$ y $\mathcal{D}_{K}(t)$ con respecto a. $N$ que no hemos llevado a cabo debido a que el interés de este trabajo se centra en el estudio del comportamiento del oscilador pesado en el estado de equilibrio, pero que esperamos continuar en futuras investigaciones.

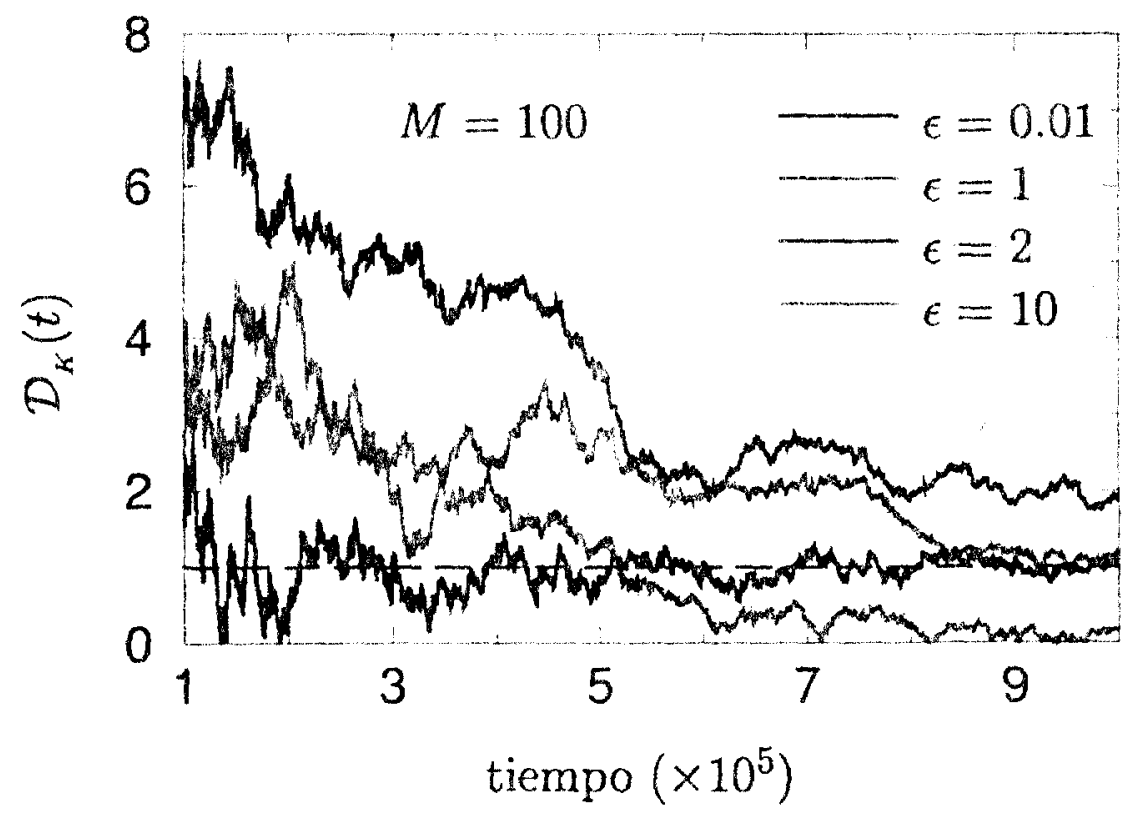

Figura 3.11: Diferencia relativa: $M=100$ y $N=300000$.

\subsection{Resultados Adicionales}

El comportamiento presentado en la sección anterior es irrelevante una vez que se ha alcanzado el estado de equilibrio termodinámico, mismo que necesitamos para estudiar el movimiento Browniano del oscilador pesado. Sin embargo, la fenomenología encontrada es lo suficientemente interesante como para merecer una investigación adicional. Desde este punto de vista, es entonces necesario hacer 
algunas suposiciones sobre el origen del comportamiento asintótico del sistema en el régimen anarmónico. Observamos en primer lugar que la única diferencia con el. modelo FPU usual es la presencia del defecto pesado en el centro de la red. Por lo tanto, la hipótesis más plausible es que los efectos hasta ahora observados pueden tener su origen en el tipo de interacción que existe entre el oscilador pesado y el resto del sistema. Para explorar esta conjetura tenemos que hacer el siguiente cambio de nomenclatura:

$$
\left(X_{i}, P_{i}\right)=\left\{\begin{array}{lll}
(X, P) & \text { si } & i=0 \\
\left(q_{i}, p_{i}\right) & \text { si } & i \neq 0
\end{array}\right.
$$

Entonces el Hamiltoniano (5.4) se puede escribir de la siguiente forma

$$
H=H_{P B}+H_{B T}+H_{I N T},
$$

donde

$$
\begin{aligned}
H_{P B} & =\frac{P^{2}}{2 M}+X^{2}+\frac{\beta}{4} X^{4} \\
H_{B T} & =\sum_{i=1}^{N} \frac{p_{i}^{2}}{2}+\sum_{i=1}^{N-1}\left[\frac{1}{2}\left(q_{i+1}-q_{i}\right)^{2}+\frac{\beta}{4}\left(q_{i+1}-q_{i}\right)^{4}\right]+\frac{1}{2}\left(q_{1}^{2}+q_{N}^{2}\right)+\frac{\beta}{4}\left(q_{1}^{4}+q_{N}^{4}\right) \\
\mathrm{y} & \\
H_{I N T} & =-\beta\left(q_{1}^{3} X-\frac{3}{2} q_{1}^{2} X^{2}+q_{1} X^{3}+X^{3} q_{N}-\frac{3}{2} X^{2} q_{N}^{2}+X q_{N}^{3}\right)-X\left(q_{1}+q_{N}\right) .
\end{aligned}
$$

$H_{P B}$ corresponde al Hamiltoniano de un oscilador anarmónico aislado, $H_{B T}$ es el Hamiltoniano de una cadena FPU de masas unitarias y extremos fijos, y finalmente $H_{I N T}$ cuantifica la interacción entre el oscilador anarmónico y la cadena FPU. Ahora bien, si definimos $h(t) \equiv H(t) / N$, entonces podemos monitorear la evolución temporal de las cantidades $h_{P B}(t), h_{B T}(t)$ y $h_{I N T}(t)$ y poder así observar la relación que existe entre ellas.

Los primeros resultados fueron obtenidos para el caso armónico con $N=2000$ y se muestran en la figura 5.12(a). Desde una perspectiva distinta volvemos a encontrar la misma fenomenología que la ya mostrada en la figura 5.3. Se observa que la energía del sistema, tanto cinética como potencial, está concentrada en el baño de osciladores livianos, siendo despreciable la contribución del oscilador pesado. Es asimismo despreciable la energía de interacción entre ambos sistemas, por lo que el oscilador pesado y la cadena de osciladores livianos están esencialmente 


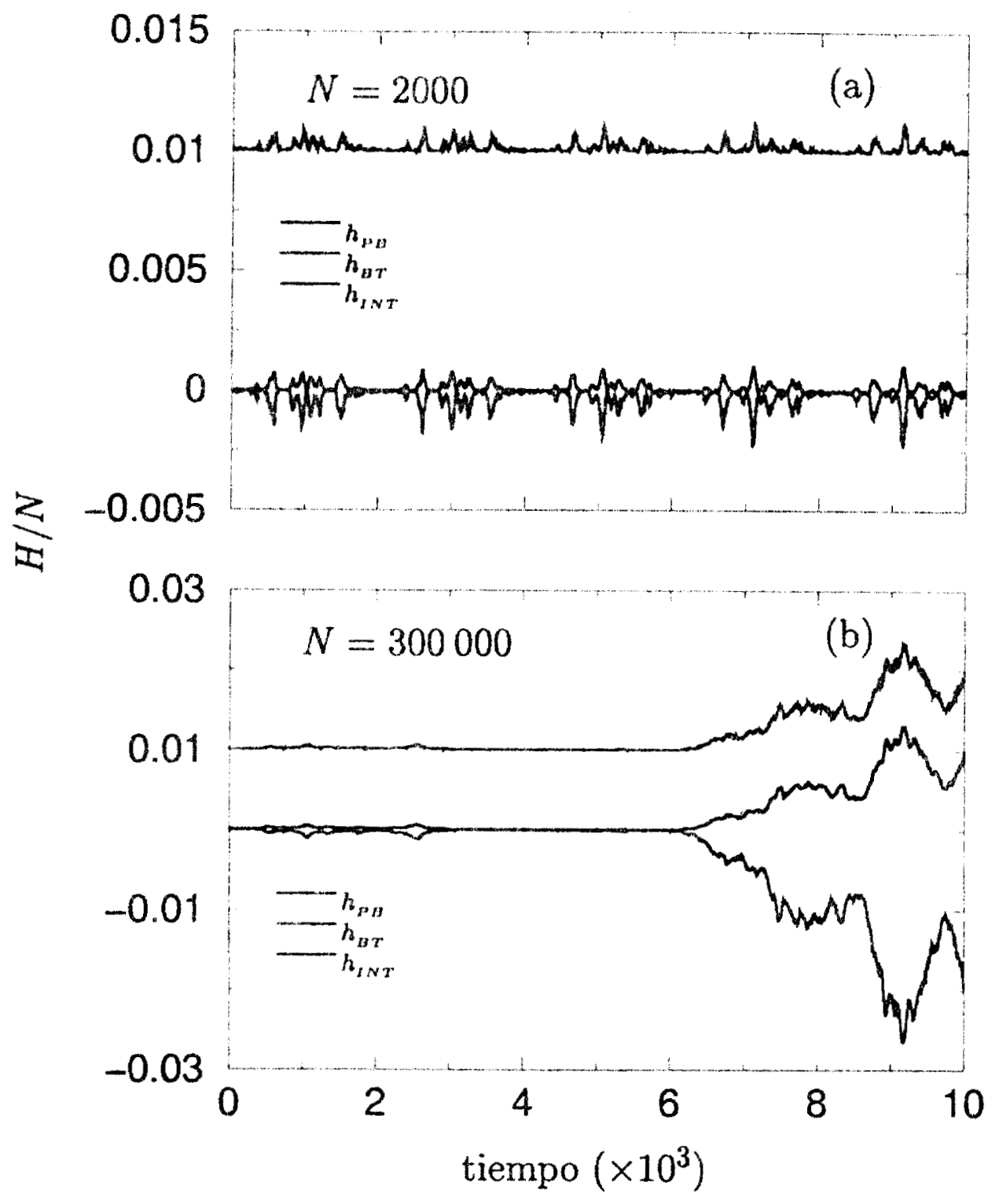

Figura 5.12: $h_{P B}, h_{B T}$ y $h_{I_{N T}}$ para el caso particular $M=60$ como función del número de osciladores livianos en el régimen armónico $(\epsilon=0.01)$.

desacoplados. La situación cambia cuando tenemos $N=300000$, como puede verse en la figura 5.12(b); para este caso, y después de un tiempo transitorio más grande que aquel encontrado en la figura 5.6, vemos claramente que la energía del oscilador pesado empieza a incrennentarse. Además, la forma de la curva cor- 
respondiente $: h_{P B}(t)$ tiende a seguir muy de cerca a la curva de la energía del baño térmico $i_{B T}$. Este comportamiento proporciona una clara evidencia de que empiezan a formarse correlaciones entre el baño y el sistema. Si aumentamos el tiempo de observación hasta $10^{5}$ tenemos que las curvas correspondientes a $h_{P B} \mathrm{y}$ $h_{B T}$ terminan por seguir una evolución temporal prácticamente idéntica, mientras que la energía de interacción toma valores negativos muy grandes, como puede verse en la figura $5 \cdot 13(\mathrm{a})$.

La situación para $\epsilon=10$ se muestra en la figura 5.13(b). Aunque existen semejanzas con el caso armónico, las diferencias resultan ser todavía más interesantes ya que, en el intervalo de tiempo mostrado, el sistema retorna a un estado semejante al inicial, de manera totalmente opuesta a lo esperado y en contraposición al caso lineal. Este comportamiento tan extraño debe de tener su explicación en la interacción que existe entre el oscilador pesado y el baño de osciladores livianos, ya que el elemento diferente que existe con respecto al caso lineal es el predominio de la contribución anarmónica en el Hamiltoniano de interacción $H_{I N T}$.

Sin embargo, el estudio de la influencia de esta peculiar interacción en la relajación hacia el equilibrio está más allá de los objetivos del presente trabajo, por lo que sólo nos conformaremos con dejar constancia de los efectos de esta interacción. Otra de las razones para no continuar con esta linea de investigación en el presente momento tiene que ver con la estructura misma de $H_{I N T}$. Nuestro modelo, (5.4), ha sido construido de tal manera que su estructura fuera muy similar al modelo de osciladores acoplados (3.1), sin preocuparnos por el problema de la interacción entre el oscilador pesado y el baño de osciladores livianos. Una consecuencia de esta metodología es que la ya aludida interacción resulta muy complicada, puesto que involucra términos no-lineales tanto en las coordenadas del oscilador pesado corno en las del baño. De hecho, parece que, hasta donde llega el conocimiento del autor de este trabajo, nunca se ha intentado el estudio de una interacción como la que está expresada en $H_{I N T}$. Pero podemos hacer algunas suposiciones razonables sobre el origen de la fenomenología que hemos encontrado. En la literatura sobre el tema han podido estudiarse sistemas simplificados en los que la interacción es lineal en las coordenadas del baño y arbitraria (esto es, nolineal) en las coordenadas del oscilador pesado $[119,120]$. En este caso se sabe que la consecuencia es la aparición de ruido multiplicativo en la dinámica macroscópica resultante. Si este mismo fenómeno está presente en nuestro sistema, entonces la ecuación de movimiento para el momento del oscilador pesado, cuando éste alcance 

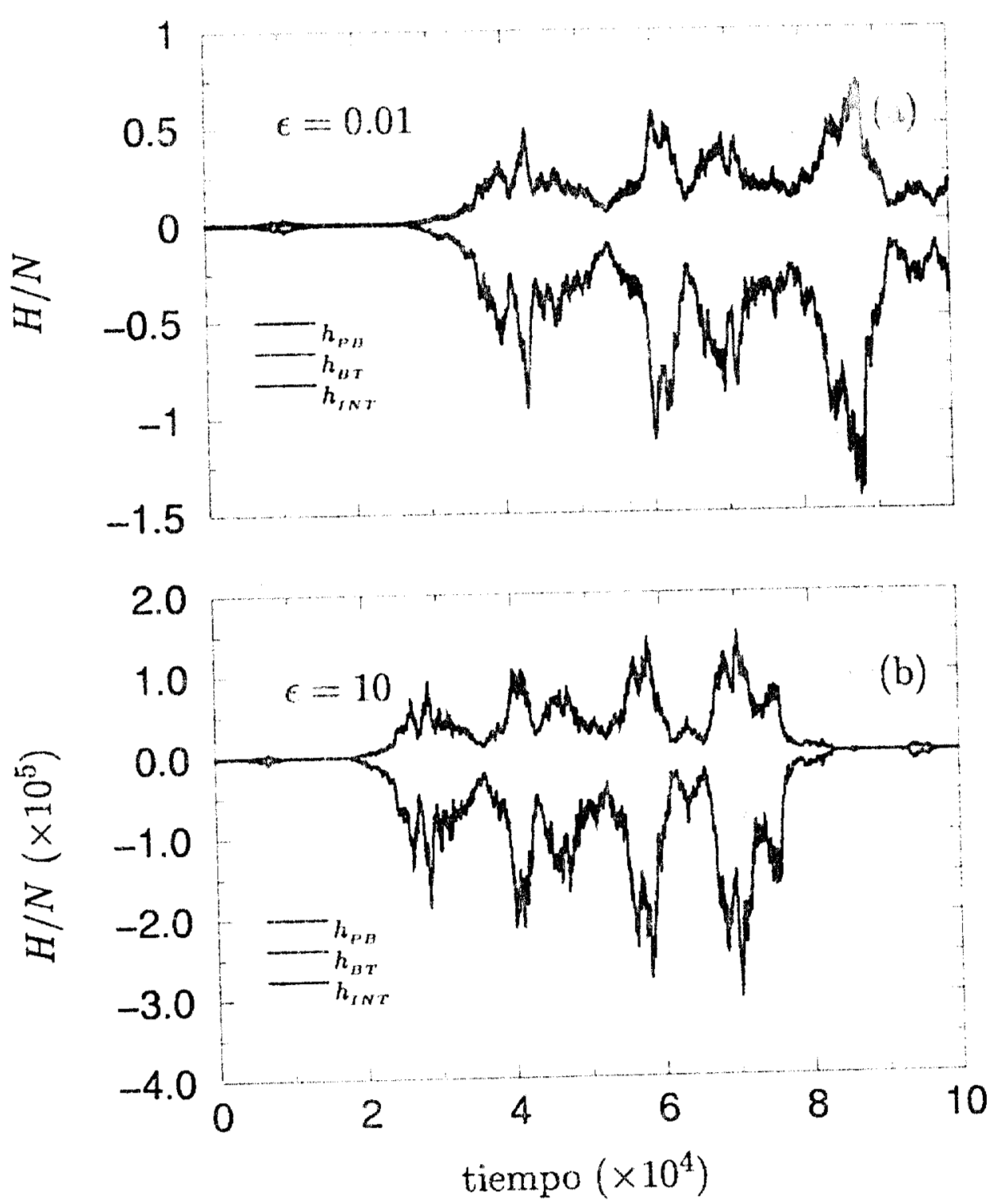

Figura 5.13: $h_{P B}, h_{v \tau}$ y $h_{I N T}$ para el caso particular $M=60$ en función de la densidad de energía $\epsilon$ y con $N=300000$ en ambos casos.

el estado de equilibrio ternodinámico, debería de tener la forma

$$
\dot{P}(t)=-\Gamma P(t)+g(P(t)) f(t)
$$

donde $g$ es una función del momento $P(t)$ tal que $g(P(t)) \rightarrow 1$ cuando $\epsilon \ll 1$. 
Las implicaciones de esta suposición son importantes, pero su esclarecimiento seŕ́ pospuesto para futuras investigaciones debido a las razones ya anteriormente. mencionadas.

\subsection{Conclusiones}

En este capitulo hemos modificado el modelo FPU- $\beta$ introduciendo un defecto pesalo de tal manera que este nuevo modelo sea una extensión del Hamiltoniano de osciladores armónicos acoplados (3.1) ya antes utilizado en el estudio del movimiento Browniano. Las ecuaciones de movimiento del modelo FPU modificado ban sido resueltas numéricamente y hemos podido comprobar la confiabilidad de las trayectorias obtenidas al comprobar la conservación de la energía, así como en el establecimiento del intervalo de valores de $\epsilon$ que corresponden al régimen ordenado y caútico de nuestro modelo. Sin embargo, hemos podido también comprobar que la construcción del estado de equilibrio definido por el principio de equipartición (5.9) es la tarea más difícil hasta este punto de nuestro desarrollo. Como resulta claro de la figura 5.6, para tiempos cortos nuestro sistema sigue una dinámica que no obedece a la ecuación de Langevin, como era de esperarse; sólo cuando las fluctuaciones relativas del baño de osciladores livianos alcanzan su valor estadístico $\sim O(1 / N)$ podemos tener la seguridad de que el sistema ha alcanzado el estado de eyuilibrio buscado. Pero esta relajación no ha resultado nada fácil de caracterizar. A través de la función $\mathcal{D}_{\kappa}(t)$ hemos podido observar que, para lo6 valores extremos de $\epsilon$ que hemos utilizado (0.01 y 10), el comportamiento es muy similar, pero a la vez muy diferente a los casos intermedios también estudiados. Este comportamiento ha impedido utilizar a $\mathcal{D}_{K}(t)$ de una forma análoga a la entropia espectral (4.7) definida en el capítulo 4 para definir un tiempo de equiparticion independiente de las fluctuaciones relativas del baño. Una posible causa de este fracaso la hemos encontrado estudiando la interacción del baño con el oscilador pesado, ya que el comportamiento en el régimen caótico es marcadamente diferente al del régimen armónico. La conjetura de que esta interacción da origen a ruido multiplicistivo en la dinámica macroscópica de nuestro modelo es plausible considerando lus resultados disponibles en la literatura para modelos similares, pero no ha sido explorada con todo el cuidado que amerita porque, a primera aproximación, hemos conseguido el principal objetivo propuesto en este capítulo, el cual era el de construir un sistema de osciladores anarmónicos que tuviera un comportamiento estadístico adecuado. Por lo tanto, las preguntas sin respuesta 
propuestas en este capítulo serán pospuestas para futuras investigaciones, ya que, con la información obtenida hasta el momento, estamos en la posición de estudiar detalladamente el movimiento del oscilador pesado para comprobar si éste efectúa movimiento Browniano de manera análoga al sistema de osciladores armónicos acoplados, lo que haremos a continuación. 


\section{Capítulo 6}

\section{Movimiento Browniano en una Cadena FPU}

\subsection{Introducción}

En el capítulo anterior planteamos el modelo FPU modificado, establecimos la manera de integrar numéricamente las correspondientes ecuaciones de movimiento y estudiamos la relajación hacia el estado de equilibrio termodinámico en función de ciertos valores de los parámetros característicos del sistema. Este proceso de relajación resultó muy difícil de estudiar debido, según los datos obtenidos, al tipo de interacción entre el oscilador pesado y el resto del sistema, por lo que la fenomenología puesta er: evidencia por nuestros experimentos numéricos todavía no ha podido ser explicada en su totalidad.

Ahora bien, a pesar de las dificultades encontradas hemos podido obtener el estado de equilibrio con un grado razonable de aproximación, por lo que podemos retomar la pregunta que ya nos planteábamos en la introducción: ¿qué modificaciones introduce en la fenomenología macroscópica la presencia de un potencial anarmónico en el Hamiltoniano microscópico del sistema? Finalmente podremos, en el presente capítulo, responder a esta pregunta de la manera menos ambigua que nos permitan los datos numéricos que obtengamos siguiendo la metodología que más adelante presentaremos.

Nuestro primer objetivo será el de estudiar el modelo ya planteado en el límite armónico de tal manera que reproduzcamos numéricamente los resultados obtenidos analíticamente en el capítulo 3. Aparte de ser una comprobación del alcance de nuestra metodología, los resultados obtenidos en este régimen tam- 
bién tienen un interés intrínseco, puesto que nunca se habian intentado reproducir numéricamente los resultados analíticos ya conocidos. Posteriormente estudiaremos las modificaciones que el potencial anarmónico introduce en los parámetros macroscópicos característicos del movimiento Browniano, principalmente en el coeficiente de difusión y en el tiempo de relajación de la función de autocorrelación del momento, además de investigar cómo calcularlos con la mayor precisión posible.

\subsection{Validación: El Caso Armónico}

Puesto que ya comprobamos exhaustivamente en el capítulo anterior que, cuando $\epsilon \ll 1$, el comportamiento del sistema definido por el Hamiltoniano (5.4) es prácticamente indistinguible de aquel obtenido utilizando (3.1) (esto es, del caso en que el potencial anarmónico es estrictamente nulo), entonces podemos iniciar muestro estudio del movimiento del oscilador pesado recuperando numéricamente los resultados ya obtenidos analíticamente en el capítulo 3 para el caso de interacciones a primeros vecinos. Con esto garantizamos que podemos estudiar, con un grado razonable de certidumbre, el comportamiento del sistema cuando tengamos que $\epsilon>1$, régimen en el cual no existen expresiones analíticas disponibles.

Para estudiar el movimiento Browniano en una cadena de osciladores utilizando las soluciones nurnéricas a las ecuaciones de movimiento, la metodología consistirá en estudiar las propiedades asociadas al oscilador pesado y que se obtienen a partir de las mismas, tales como su posición, momento y la fuerza total ejercida sobre el oscilador pesado debido a su interacción con todos los demás osciladores del sistema. Puesto que a partir de cada una de estas variables se pueden obtener relaciones bien conocidas, la comprobación del comportamiento estadístico en el régimen armónico se vuelve inmediata, como a continuación veremos.

\subsubsection{Desplazamiento Cuadrático Medio}

Para el caso de la posición, tenemos que comprobar si en nuestro modelo se cumple la relación de difusión de Einstein (2.10). Esto es, debemos obtener una relación del tipo

$$
\left\langle\left[X_{0}(t)-X_{0}(0)\right]^{2}\right\rangle_{t}=2 D t
$$

para $t \gg \tau$, donde $\tau$ es el tiempo caracteristico de decaimiento de la función de autocorrelación del momento. En esta expresión el promedio está calculado en un 
estado de equilibrio termodinámico, por lo que no depende del origen temporal y se dice entonces que el prornedio es estacionario. Esta última propiedad nos permite calcular, para una función $\Psi(t, 0)$ arbitraria (en este caso particular, $\Psi(t, 0)=$ $\left.\left[X_{0}(t)-X_{0}(0)\right]^{2}\right)$, el promedio $\langle\Psi(t, 0)\rangle_{t}$ como $[121]$

$$
\langle\Psi(t, 0)\rangle_{t}=\frac{1}{\mathcal{N}} \sum_{n}^{\mathcal{N}} \Psi\left(t_{n}+t, t_{n}\right),
$$

donde $t_{n}$ son los orígenes temporales que pueden obtenerse a partir de una trayectoria de longitud $t_{\max }$ y cuyo número total disponible está dado por $\mathcal{N}=t_{\max }-t^{1}$. Ahora bien, como el promedio (6.2) tiene que evaluarse sobre la trayectoria del oscilador masivo, entonces la precisión del promedio sólo puede ser aumentada extendiendo lo más posible la trayectoria en el tiempo. Empíricamente hemos encontrado que $t_{\max }=2 \times 10^{5}$ es el tamaño óptimo de la trayectoria (con lo que obtenemos aproximadamente 199800 orígenes temporales descorrelacionados) para una correcta evaluación del promedio $\langle\cdots\rangle_{t}$ en $(6.1)$.

Una estimación del valor del coeficiente de difusión $D$ se puede obtener a partir de (2.34), conocida en la literatura como fórmula de Green-Kubo, si la reescribimos en términos de la función de autocorrelación del momento $\rho_{0}(t)$, con lo que resulta la expresión

$$
M^{2} D=\int_{0}^{\infty}\left\langle P_{0}^{2}(0)\right\rangle \rho_{0}(t) d t
$$

Si utilizamos el principic de equipartición $\left(\left\langle P_{0}^{2}(0)\right\rangle=M k_{B} T\right)$ y la forma exponencial asintótica $\rho_{0}(t)=\exp (-\Gamma t)$, donde $\Gamma=m \omega_{0} / M\left(\omega_{0}=2\right.$ y $m=1$ en nuestro sistema de unidades), entonces obtenemos la relación $2 D=k_{B} T$, misma que fue calculada de manera exacta para el modelo de osciladores armónicos acoplados [45]. Ahora bien, si calculamos la temperatura en unidades de la constante de Boltzmann, tenemos que $2 D$ tiene que coincidir con la temperatura cinética $T_{c i n}$ que obtuvimos en el capítulo anterior. Puesto que el desplazamiento cuadrático medio es la cantidad más fácilmente medible por medio de la dinámica molecular, tenemos entonces una forma muy sencilla de calcular el coeficiente de difusión $D$ del oscilador pesado. Además, como puede también calcularse a partir de la fórmula de Green-Kubo (2.34), tenemos, en principio, una forma adicional de comprobar su validez. Mencionemos por último que, aparte del límite $t \gg \tau \equiv \Gamma^{-1}$,

\footnotetext{
${ }^{1}$ Cabe mencionar que, en virtud del teorema ergódico, esta forma de calcular el promedio $\langle\cdots\rangle_{t}$ es equivalente a tomar un ensamble de sistemas idénticos excepto por sus condiciones iniciales, que son diferentes para cada miembro del ensamble.
} 


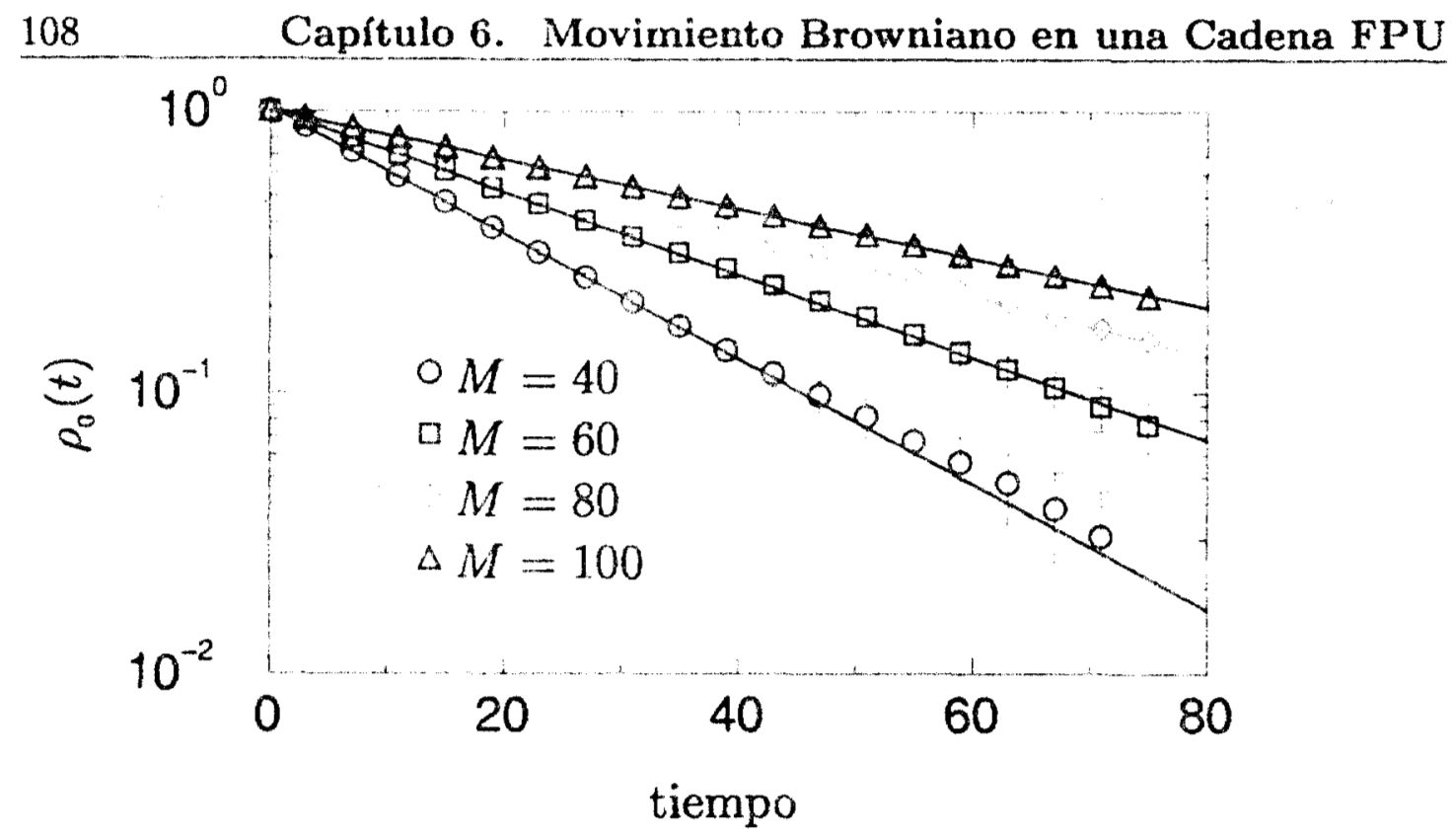

Figura 6.3: Logaritmo de la función de autocorrelación normalizada del momento para cada una de las masas utilizadas vs tiempo. Mismos parámetros que en la figura 6.2.

en una escala lineal-logarítmica. El comportamiento lineal de las curvas en esta gráfica indica claramente un decaimiento exponencial de la forma $\rho_{0}(t)=$ $\exp (-t / \tau)$ en el régimen temporal mostrado, lo que confirma que la ecuación de Langevin (3.39) proporciona una descripción adecuada a la fenomenología hasta ahora estudiada.

Una forma adicional de comprobar la validez de nuestros resultados consistirá en calcular el tiempo de relajamiento $\tau$ a partir de (6.6). Ahora bien, es claro que el límite superior infinito de la integral es meramente formal; tenemos que sustituirlo por un tiempo máximo para el cual la función $\rho_{0}(t)$ ya haya decaido apreciablemente. Este tiempo lo podemos tomar del límite superior del intervalo de validez (6.4) para cada una de las masas estudiadas. Los resultados se muestran en la tabla 6.2 , junto con el correspondiente coeficiente de difusión calculado directamente a partir de la fórmula de Green-Kubo (2.34).

Como resulta evidente, el cálculo del tiempo de relajamiento resulta ser excelente, sobre todo para los casos $M=40$ y 60 . En ambos el error porcentual con respecto al valor exacto es despreciable, $\sim 6.5 \times 10^{-5} \%$. En los otros dos casos el resultado numérico está ligeramente más alejado del valor exacto, y esto es muy probablemente debido a la desviación sistemática que ya habíamos notado 
estado de equilibrio termodinámico, por lo que no depende del origen temporal y se dice entonces que el promedio es estacionario. Esta última propiedad nos permite calcular, para una función $\Psi(t, 0)$ arbitraria (en este caso particular, $\Psi(t, 0)=$ $\left.\left[X_{0}(t)-X_{0}(0)\right]^{2}\right)$, el promedio $\langle\Psi(t, 0)\rangle_{t}$ como $[121]$

$$
\langle\Psi(t, 0)\rangle_{t}=\frac{1}{\mathcal{N}} \sum_{n}^{\mathcal{N}} \Psi\left(t_{n}+t, t_{n}\right)
$$

donde $t_{n}$ son los orígenes temporales que pueden obtenerse a partir de una trayectoria de longitud $t_{\max }$ y cuyo número total disponible está dado por $\mathcal{N}=t_{\max }-t^{1}$. Ahora bien, como el promedio (6.2) tiene que evaluarse sobre la trayectoria del oscilador masivo, entonces la precisión del promedio sólo puede ser aumentada extendiendo lo más posible la trayectoria en el tiempo. Empíricamente hemos encontrado que $t_{\max }=2 \times 10^{5}$ es el tamaño óptimo de la trayectoria (con lo que obtenemos aproximadamente 199800 orígenes temporales descorrelacionados) para una correcta evaluación del promedio $\langle\cdots\rangle_{t}$ en $(6.1)$.

Una estimación del valor del coeficiente de difusión $D$ se puede obtener a partir de (2.34), conocida en la literatura como fórmula de Green-Kubo, si la reescribimos en términos de la función de autocorrelación del momento $\rho_{0}(t)$, con lo que resulta la expresión

$$
M^{2} D=\int_{0}^{\infty}\left\langle P_{0}^{2}(0)\right\rangle \rho_{0}(t) d t
$$

Si utilizamos el principio de equipartición $\left(\left\langle P_{0}^{2}(0)\right\rangle=M k_{B} T\right)$ y la forma exponencial asintótica $\rho_{0}(t)=\exp (-\Gamma t)$, donde $\Gamma=m \omega_{0} / M\left(\omega_{0}=2\right.$ y $m=1$ en nuestro sistema de unidades), entonces obtenemos la relación $2 D=k_{B} T$, misma que fue calculada de manera exacta para el modelo de osciladores armónicos acoplados [45]. Ahora bien, si calculamos la temperatura en unidades de la constante de Boltzmann, tenemos que $2 D$ tiene que coincidir con la temperatura cinética $T_{c i n}$ que obtuvimos en el capítulo anterior. Puesto que el desplazamiento cuadrático medio es la cantidad más fácilmente medible por medio de la dinámica molecular, tenemos entonces una forma muy sencilla de calcular el coeficiente de difusión $D$ del oscilador pesado. Además, como puede también calcularse a partir de la fórmula de Green-Kubo (2.34), tenemos, en principio, una forma adicional de comprobar su validez. Mencionemos por último que, aparte del límite $t \gg \tau \equiv \Gamma^{-1}$,

\footnotetext{
${ }^{1}$ Cabe mencionar que, en virtud del teorema ergódico, esta forma de calcular el promedio $\langle\cdots\rangle_{t}$ es equivalente a tomar un ensamble de sistemas idénticos excepto por sus condiciones iniciales, que son diferentes para cada miembro del ensamble.
} 
no es conocido con precisión el instante en el que (6.1) se vuelve una aproximación válida, ni el tamaño del intervalo temporal en el cual mantiene su validez, por lo que la estimación de $2 D$ obtenida en este párrafo se vuelve de gran utilidad. Para las distintas masas estudiadas se presenta el resultado del cálculo del desplazamiento cuadrático medio en la figura 6.1 .

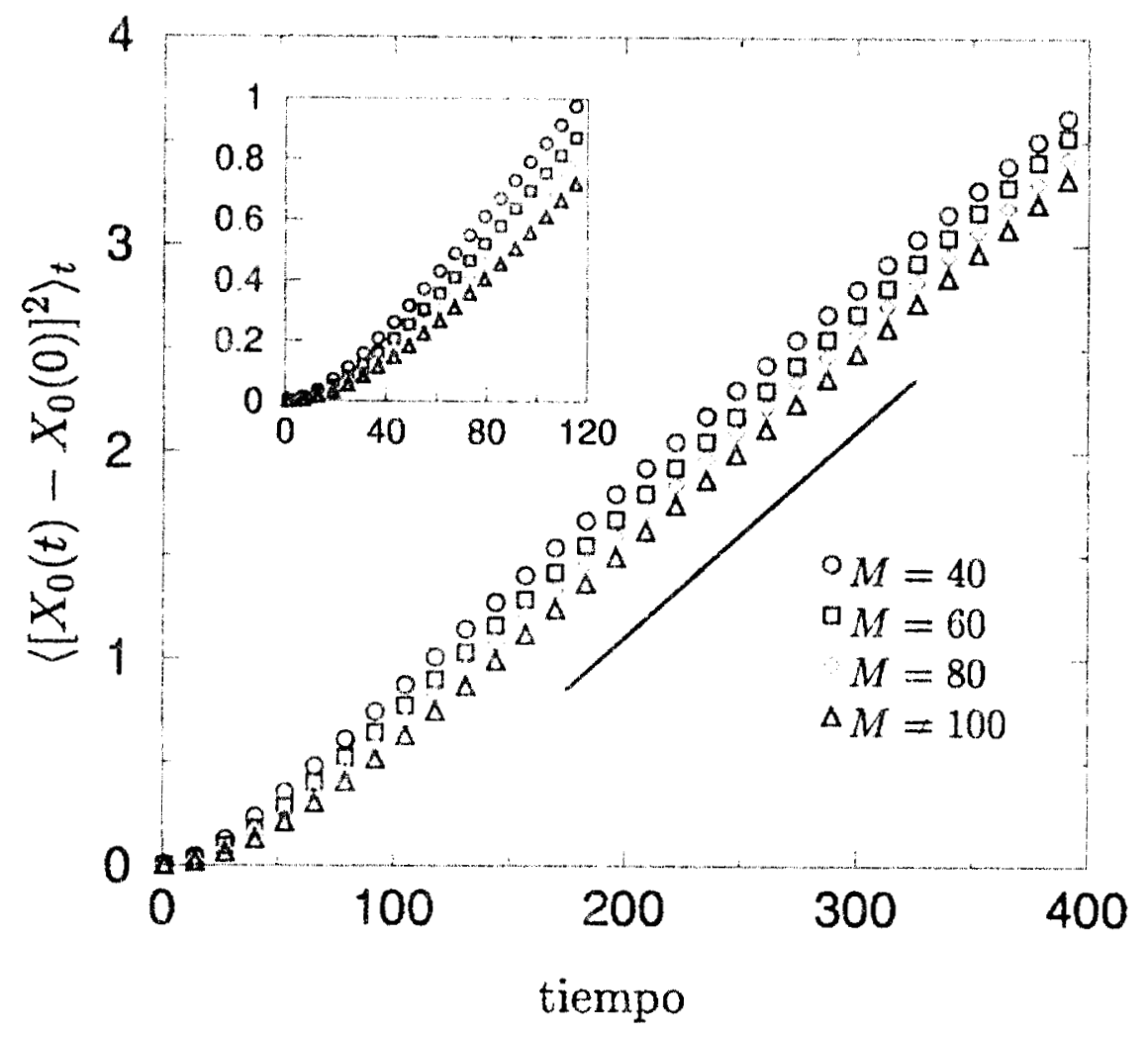

Figura 6.1: Desplazamiento cuadrático medio del oscilador pesado en el límite armónico $(\epsilon=0.01)$ con $N=300000$ para todos los valores de $M$ utilizados. En la figura pequeña se muestra, para mayor claridad, el comportamiento balístico $\sim t^{2}$ que es dominante cuando $t \ll \tau$ en todos los casos. La recta continua, mostrada como referencia, tiene una pendiente de 0.01 .

Como resulta evidente, el comportamiento asintótico, que corresponde a tener lineas rectas que sean muy aproximadamente paralelas, es el que esperábamos. En general tenernos que $\tau=M / 2 \mathrm{y}$, para la masa más grande de $M=100, \tau=50$, por lo que podemos considerar que, para $t \geq 100$, ya estamos en el régimen $t \gg \tau$ en el que la relación de Einstein (6.1) es válida. Si eliminamos los primeros 100 
puntos de cada una de las rectas y se ajusta su pendiente por medio de una regresión de mínimos cuadrados, obtenemos inmediatamente los "coeficientes de difusión" $D_{M}$. Estos resultados están reportados en la tabla 6.1.

Tabla 6.1: Coeflciente de Difusión

\begin{tabular}{|c|cccc|}
\hline & \multicolumn{4}{c|}{$M$} \\
\hline & $\mathbf{4 0}$ & $\mathbf{6 0}$ & $\mathbf{8 0}$ & $\mathbf{1 0 0}$ \\
\hline$D_{M}{ }^{a}$ & 0.0052 & 0.0053 & 0.0052 & 0.0052 \\
\hline
\end{tabular}

${ }^{a}$ En todos los casos las incertidumbres asociadas son $\sim O\left(10^{-5}\right)$

Como ya vimos en el tratamiento fenomenológico del capítulo 2 , el coeficiente $D$ no depende de la masa de la partícula Browniana; esta propiedad también es válida para el caso del oscilador pesado en una cadena infinita de osciladores armónicos interaccionando a primeros vecinos [45] (recordar que ya habíamos establecido que $D=k_{B} T / 2$ ). Ahora bien, dentro del margen de incertidumbre disponible, los datos de la tabla 6.1 confirman satisfactoriamente la afirmación anterior, ya que la diferencia entre cada valor $D_{M}$ de la tabla 6.1 y la media aritmética $\bar{D}$ no es estadísticamente significativa, por lo que podemos hacer la identificación $D \approx \bar{D}$. Además de proporcionar una comprobación adicional de la validez de nuestra metodología, este resultado es un gran avance con respecto al único trabajo semejante al nuestro existente en la literatura. Omerti et al. [122] estudiaron un sistema unidimensional de partículas puntuales en el que una de ellas tiene una masa mayor que las demás, encontrando una dependencia del coeficiente de difusión de la partícula masiva con respecto a su masa, lo cual es un resultado físicamente poco razonable.

\subsubsection{Autocorrelación del Momento}

Nuestro siguiente objetivo será calcular la función de autocorrelación del momento $\rho_{0}(t)$ para el oscilador pesado, ya que es una de las cantidades más fundamentales que describen al movimiento Browniano, como tuvimos ocasión de ver en el capítulo 3. De la relación (3.42), que reescribimos como

$$
\frac{1}{2}<t<\frac{M}{2} \ln M
$$

sabemos que sólo en este intervalo se mantendrá la forma exponencial de $\rho_{0}(t)$, por lo que, de momento, despreciaremos el comportamiento asintótico de la misma. Sin embargo, en ese intervalo la aproximación tiene que tener un alto grado 
de precisión, ya que posteriomente haremos comparaciones con los diversos casos anarmónicos. El método para calcular la función de autocorrelación del momonto es el mismo que aquel ya utilizado para el desplazamiento cuadrático medio. Pero ahora, debido a que $\rho_{0}(t)$ es una función decreciente, es posible calcular las incertidumbres que son introducidas al aproximar el promedio infinito $\langle\cdots\rangle_{\infty}$ por medio de un promedio sobre una cantidad finita de pasos de integración. Si se supone además que la cantidad a estudiar $\left(P_{0}(t)\right.$ en este caso) es una variable aleatoria con una distribución Gaussiana, podemos entonces utilizar la estimación de Zwanzig y Ailawaldi [123] para la incertidumbre de $\rho_{0}(t)$, que corresponde esencialmente a la variancia y que está dada por la expresión

$$
\sigma(t) \simeq\left(\frac{2 \tau}{t_{\max }}\right)^{\frac{1}{2}}\left[1-\rho_{0}(t)\right],
$$

donde $t_{\text {max }}$ es la longitud del intervalo temporal sobre el cual fueron obtenidos los datos para calcular la función $\rho_{0}(t)\left(2 \times 10^{5}\right.$ en nuestro caso) y $\tau$ es el tiempo característico de relajación de esa misma función y que se calcula como

$$
\tau=2 \int_{0}^{\infty} \rho_{0}^{2}(t) d t .
$$

Es importante ahora hacer algunos comentarios. Para nuestro problema particular, la suposición de Gaussianidad para $P_{0}(t)$ deja de serlo, puesto que esa condición la hemos impuesto por construcción a nuestro sistema a través de las condiciones iniciales. Además, como estamos en el régimen armónico $(\epsilon \ll 1)$ esta propiedad se mantiene para todo tiempo posterior. Entonces el tiempo de relajamiento calculado por medio de la Ec. (6.6) coincide con $\Gamma^{-1}$, el cual puede calcularse de manera exacta, como ya fue mostrado anteriormente.

En la figura 6.2 se muestran las funciones $\rho_{0}(t)$ para los valores de $M$ que hasta ahora estudiados. Usando como comparación la fórmula exacta (3.23) en cada caso vemos que la aproximación numérica es muy buena en todos los casos. Sin embargo, el caso $M=40$ muestra un comportamiento asintótico oscilatorio muy marcado que no es del todo explicable, ya que hemos comparado los resultados numéricos de la expresión exacta (3.23) con aquellos obtenidos con la forma asintótica (3.30) y ninguno de ellos muestra el comportamiento oscilatorio esperado, por lo que sería lógico suponer que este tipo de comportamiento no debería ser detectable numéricamente. Pero, como esta fenomenología ocurre para tiempos mucho mayores que el límite de validez para el caso $M=40$ podemos, en una primera instancia, ignorarla. Ahora bien, conforme aumenta la masa del oscilador 


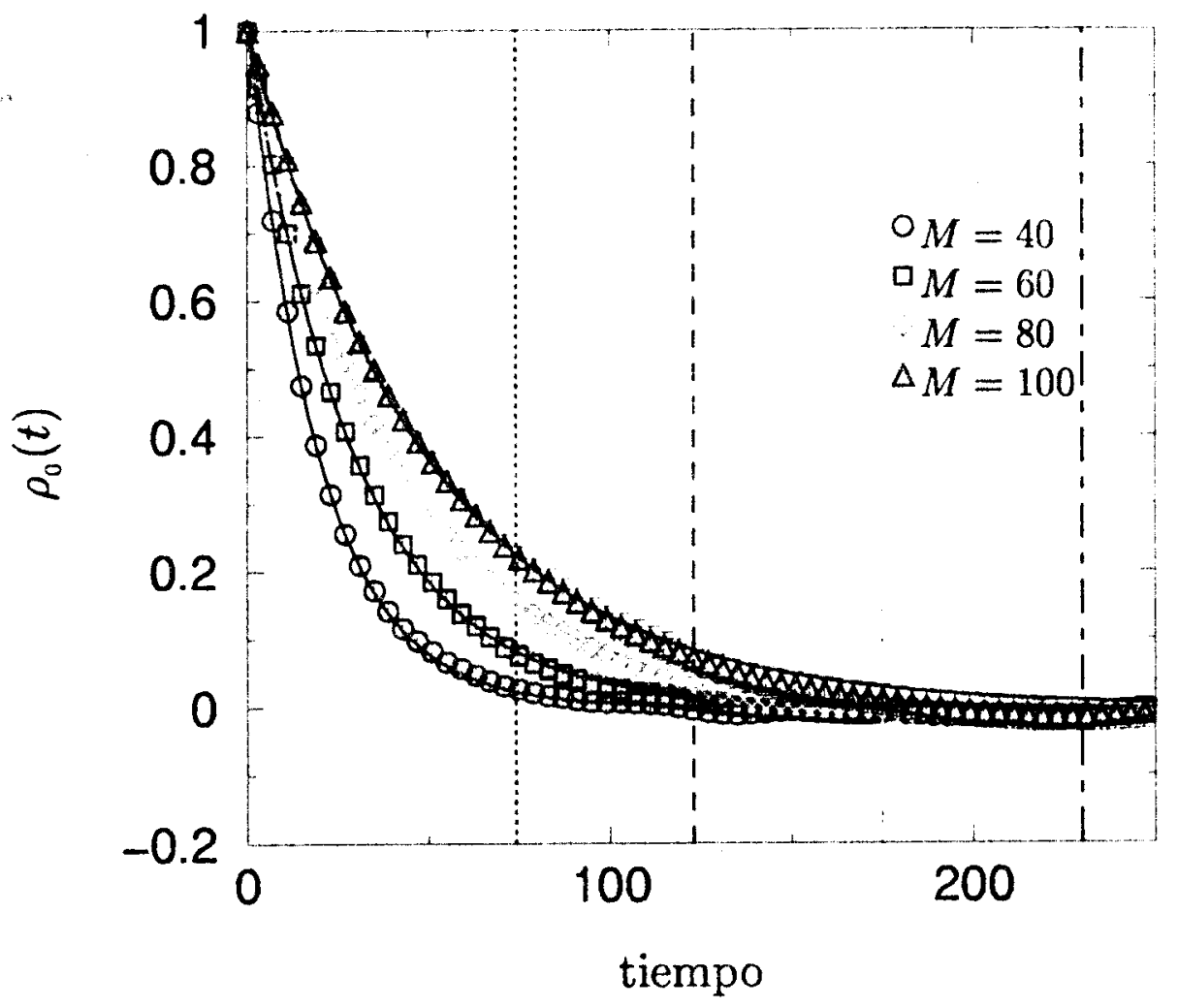

Figura 6.2: Autocorrelación del momento para cada una de las masas utilizadas en el régimen armónico $(\epsilon=0.01)$ con $N=300000$. Las lineas verticales muestran, de izquierda a derecha, el límite del intervalo de validez para $M=40,60,80,100$. Las curvas continuas corresponden a la expresión exacta (3.23) evaluada para cada una de las masas.

pesado el comportamiento asintótico ya antes señalado va desapareciendo gradualmente, aunque hay que aclarar que la incertidumbre también va aumentando y además empieza a haber una desviación sistemática con respecto a la expresión exacta incluso dentro del intervalo de validez que es particularmente evidente para $M=100$. A pesar de estos inconvenientes para todos estos casos el ajuste sigue siendo bastante bueno dentro de la incertidumbre existente, por lo que podemos considerar que efectivamente estamos calculando, a partir de los datos proporcionados por nuesto cálculo de dinámica molecular, la función de autocorrelación del momento correcta en todos los casos que hernos estudiado.

En la figura 6.3 se muestran los mismos resultados que en la figura 6.2, pero 


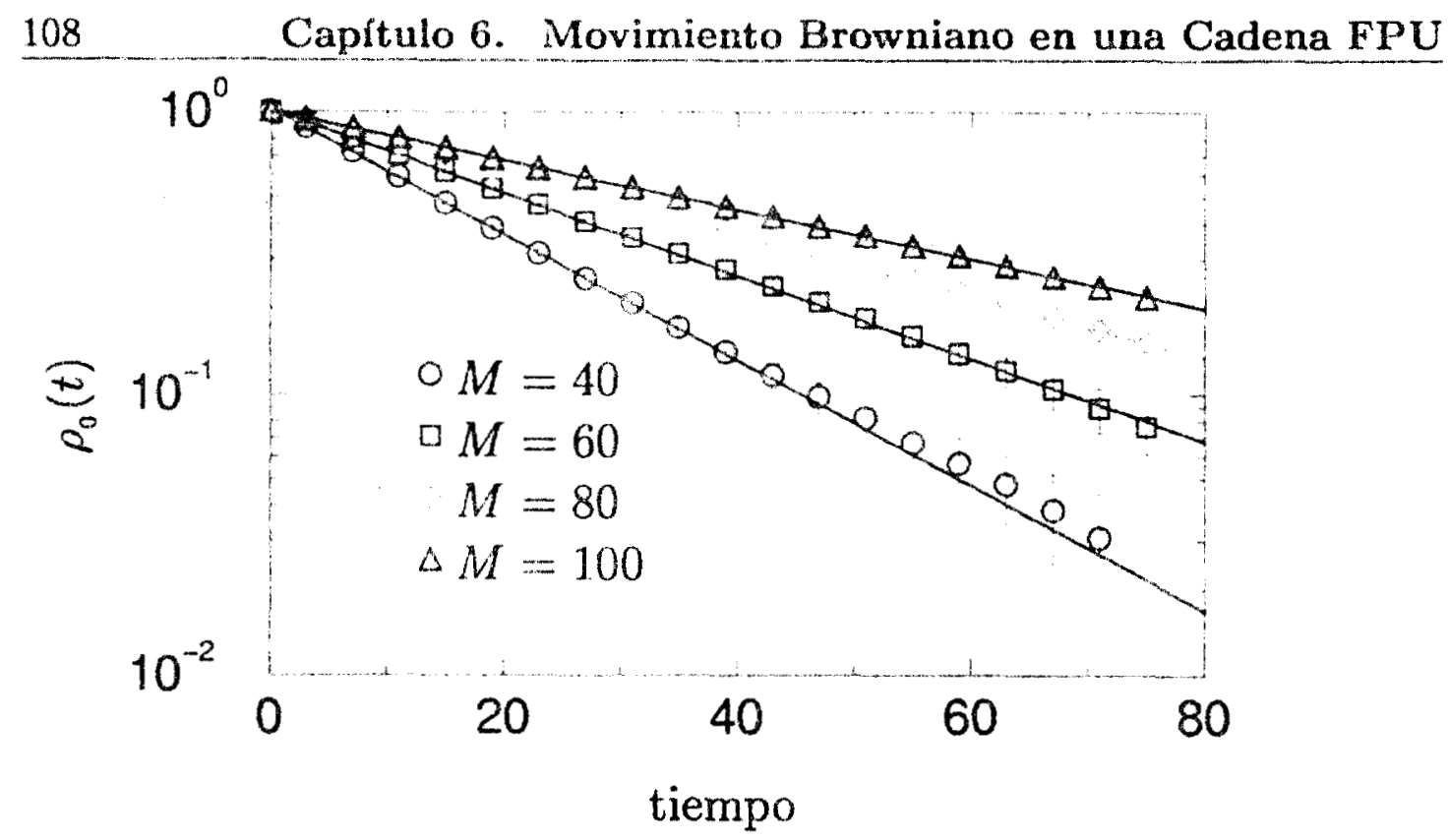

Figura 6.3: Logaritmo de la función de autocorrelación normalizada del momento para cada una de las masas utilizadas vs tiempo. Mismos paranetros que en la figura 6.2.

en una escala lineal-logarítmica. El comportamiento lineal de las curvas en esta gráfica indica claramente un decaimiento exponencial de la forma $\rho_{0}(t)=$ $\exp (-t / \tau)$ en el régimen temporal mostrado, lo que confirma que la ecuación de Langevin (3.39) proporciona una descripción adecuada a la fenomenología hasta ahora estudiada.

Una forma adicional de comprobar la validez de nuestros resultados consistirá en calcular el tiempo de relajamiento $\tau$ a partir de (6.6). Ahora bien, es claro que el límite superior infinito de la integral es meramente formal; tenemos que sustituirlo por un tiempo máximo para el cual la función $\rho_{0}(t)$ ya haya decaido apreciablemente. Este tiempo lo podemos tomar del límite superior del intervalo de validez (6.4) para cada una de las masas estudiadas. Los resultados se muestran en la tabla 6.2 , junto con el correspondiente coeficiente de difusión calculado directamente a partir de la fórmula de Green-Kubo (2.34).

Como resulta evidente, el cálculo del tiempo de relajamiento resulta ser excelente, sobre todo para los casos $M=40$ y 60 . En ambos el error porcentual con respecto al valor exacto es despreciable, $\sim 6.5 \times 10^{-5} \%$. En los otros dos casos el resultado numérico está ligeramente más alejado del valor exacto, y esto es muy probablemente debido a la desviación sistemática que ya habíamos notado 
Tabla 6.2: Tiempo de Relajación y Coeflciente de Difusión

\begin{tabular}{|l|cccc|}
\hline & \multicolumn{4}{c|}{} \\
\hline & $\mathbf{4 0}$ & $\mathbf{6 0}$ & $\mathbf{8 0}$ & $\mathbf{1 0 0}$ \\
\hline$T$ & 20.0013 & 30.0019 & 39.9906 & 49.6926 \\
\hline$D_{M}{ }^{6}$ & 0.0049 & 0.0049 & 0.0048 & 0.0047 \\
\hline
\end{tabular}

${ }^{a}$ Las incertidumbres son $\sim O\left(10^{-6}\right)$

en la figura 6.2. Pero incluso para el caso más impreciso, $M=100$, el error porcentual es de $0.61 \%$, lo cual es una aproximación todavía muy buena. Podemos concluir entonces que el cálculo del tiempo de relajación $\tau$ es confiable dentro de unos límites bastante aceptables. Sin embargo, la situación cambia para el caso del coeficiente de difusión, ya que los datos muestran una desviación sistemática negativa con respecto a aquellos calculados por medio del desplazamiento cuadrático medio, además de que esta diferencia tiende a aumentar conforme aumenta la masa del oscilador pesado. Las diferencias porcentuales están en este caso entre $5.77 \%$ y $9.62 \%$, por lo que su confiabilidad es menor con respecto a los datos de la tabla 6.1. Debido a esto es que el cálculo del coeficiente $D$ se hará utilizando el desplazamiento cuadrático medio cuando más adelante estudiemos los diversos casos en el régimen anarmónico.

\subsubsection{Fuerza sobre el Oscilador Pesado}

Como última prueba de validez calcularemos la función de autocorrelación normalizada de la fuerza instantánea $f(t)$ (FACF) que experimenta el oscilador pesado debido a la presencia del baño de osciladores livianos. De la expresión (3.36) tenemos que debe de cumplirse la relación

$$
\frac{\langle f(t) f(0)\rangle}{\left\langle f^{2}(0)\right\rangle}=J_{0}\left(\omega_{0} t\right)+J_{2}\left(\omega_{0} t\right)
$$

en el régimen armónico. Este comportamiento es precisamente lo que se observa en la figura 6.4 para el caso específico de $M=60$ con un tiempo total de cómputo de $t_{\max }=10^{5}$. Ahora bien, como ya habíamos mencionado en el capítulo 3 , Nakazawa [45] estimó que, tomando un granulamiento grueso temporal en el que la menor escala de tiempo sea $\sim 20 / \omega_{0}$, la expresión anterior se comporta muy aproximadamente como una función $\delta(t)$. En nuestro sistema de unidades la estimación anterior corresponde a tomar la menor escala de tiempo como $t \simeq 10$ 
y entonces resulta claro que, asintóticamente, queda confirmada la afirnación de Nakazawa.

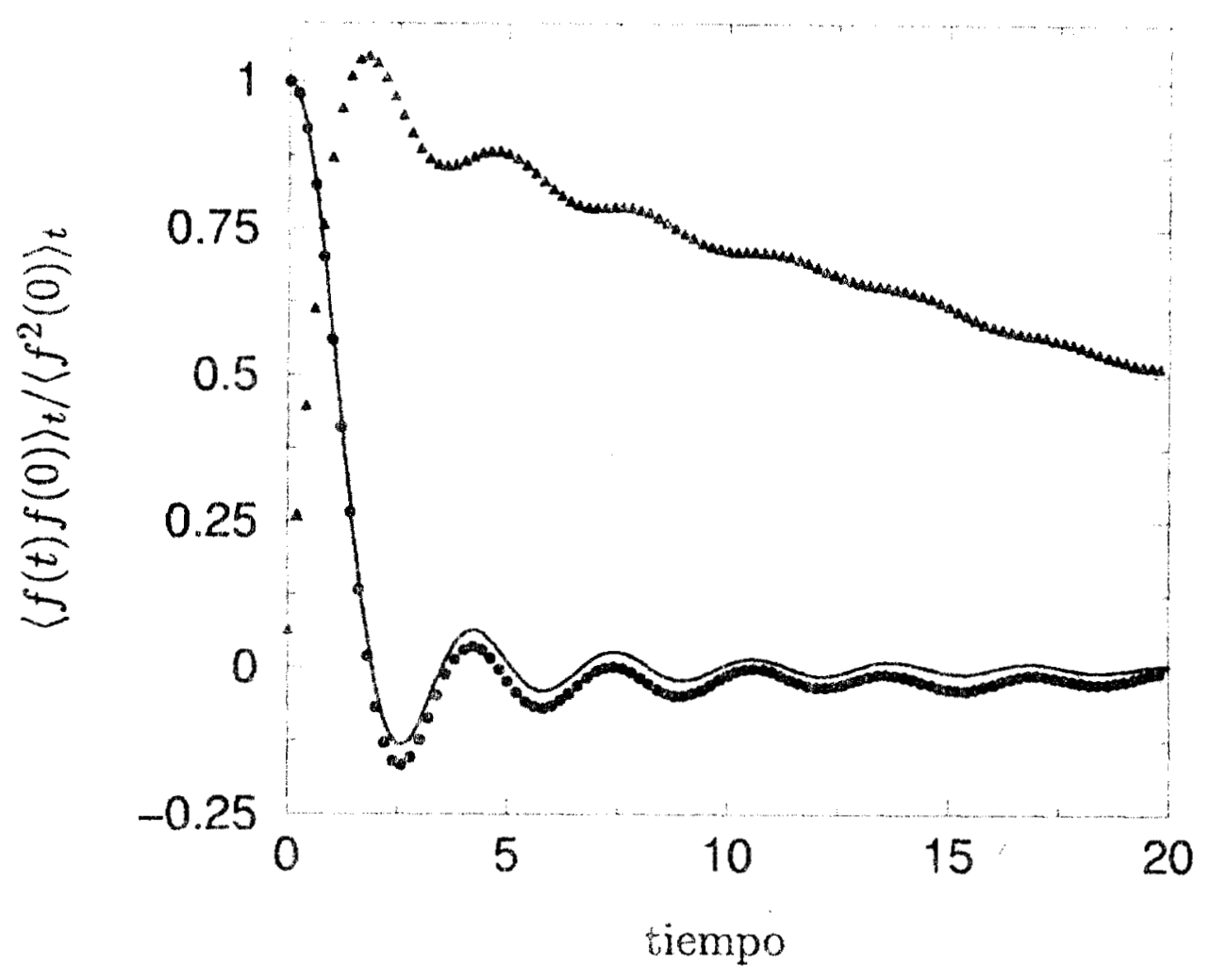

Figura 6.4: La curva continua corresponde a la expresión exacta (6.7) de la función normalizada de autocorrelación de la fuerza y los circulos son el resultado obtenido a partir de los datos de nuestra dinámica molecular. Se muestra además la integral de la FACF (triángulos). Los parámetros son $M=60$ y $N=300000$.

Puede observarse que el ajuste de los datos numéricos con respecto a (6.7) no es tan bueno como en el caso de la autocorrelación del momento, ya que el resultado exacto que estarnos utilizando ess estrictamente válido sólo para $N \rightarrow \infty$. Esta observación está basada en el hecho de que las diferencias observadas están claramente mas allá de la incertidumbre $\sigma$ de los datos numéricos, ya que $\sigma \simeq$ $\sqrt{2 t_{1} / t_{\max }} \simeq 0.005$, donde $t_{1} \simeq 1.25$ es el tiempo característico de decaimiento de la FACE (tiempo en que esta función decae desde uno hasta $\mathrm{e}^{-1}=0.368$ ). Aún así la información proporcionada por nuestro cálculo es significativa, ya que reproduce chalitativamente la forma de la curva correspondiente al resultado exacto. Es clara 
también la separación de escalas de tiempo inherente al movimiento Browniano, pues $t_{1}$ es mucho menor que el correspondiente tiempo asociado al decaimiento de $\rho_{0}(t), \tau=30$ para el caso $M=60$. Se muestra además la integral de la FACF, la cual, en principio, nos debería poder permitir calcular el coeficiente de fricción $\gamma$ a partir de

$$
\gamma=\frac{1}{T_{\text {cin }}} \int_{0}^{t_{2}}\langle f(t) f(0)\rangle d t ; \quad t_{1}<t_{2} \ll \tau .
$$

Como ya habíamos mencionado en el capítulo 2, esta integral tiende a cero cuando $t \gg \tau$ debido al hecho de que estamos trabajando con un sistema finito de osciladores. El límite superior de la integral corresponde al tienupo $\tau_{k}$ postulado por Kirkwood para el modelo de fluido, mismo que en principio permite obtener un valor finito de la integral de la FACF y, por lo tanto, del coeficiente de fricción $\gamma$. Sin embargo, como la FACF presenta un comportamiento oscilatorio muy pronunciado en el intervalo $0<t<7.5$, la correspondiente integral (6.8) no muestra la "meseta" que sería necesaria para poder calcular el coeficiente de fricción y, por el contrario, muestra un decaimiento muy pronunciado en el intervalo temporal mostrado. Esta es una limitación de nuestro modelo, ya que, como este comportamiento oscilatorio no está presente en un fluido de Lennard-Jones, para este último caso sí es posible calcular $\gamma$ a partir de $(6.8)[36,37]$. Pero la situación no es tan grave como parece a primera vista, ya que el coeficiente de fricción y el de difusión están relacionados a través de la relación $(2.35), D=\left(k_{B} T\right) \gamma^{-1}$, por lo que un cálculo basado en la integral (6.8) no ofrece mucha información adicional aparte de aquella ya obtenida anteriormente.

\subsection{Resultados en el Régimen Anarmónico}

Habiendo comprobado las capacidades de nuestro esquema para recuperar de manera numérica los resultados analíticos ya conocidos para $\epsilon \ll 1$ procederemos a continuación a explorar el comportamiento del coeficiente de difusión y del tiempo de relajación para el régimen caótico; esto es, para $\epsilon>1$. Los resultados de esta sección constituyen la parte más importante de este trabajo, ya que el comportamiento de nuestro modelo en el régimen caótico es totalmente desconocido hasta ahora. Además, teniendo como punto de comparación los casos armónicos ya estudiados se puede afirmar que cualquier desviación del comportamiento armónico proviene de la contribución del potencial cuártico en (5.4). 


\subsubsection{Coeficiente de Difusión}

Para el desplazamiento cuadrático medio obtenemos los resultados que se muestran en la figura 6.5 para algunos valores representativos de $\epsilon$. Como es evidente, para todos los casos ilustrados el intervalo temporal en el que el oscilador masivo todavía no tiene un comportamiento difusivo normal (esto es, que su desplazamiento cuadrático medio tenga comportamiento balístico) es muy semejante al del caso armónico, que habíamos estimado como $t \approx(0-100)$. Asintóticamente observamos que, para los valores extremos de $\epsilon(0.01$ y 10$)$, el desplazamiento cuadrático medio tiene un comportaniento muy similar para estos dos valores de $\epsilon, \mathrm{y}$ a su vez ligeramente distinto que el correspondiente a los casos $\epsilon=0.6$ y 1 . En este punto de nuestro desarrollo todavía no podemos aventurar una hipótesis que explique este comportamiento; nos limitamos a constatar su existencia. Ahora bien, examinando el comportamiento del desplazamiento cuadrático medio para cada uno de los valores de $\epsilon$ considerados, encontramos que en todos ellos podemos considerar que el comportamiento balístico es dominante cuando $t<100$, mientras que el mejor ajuste lineal se consigue en el intervalo $(100-400)$. Podemos entonces calcular el valor de un "coeficiente de difusion" $D_{M}$ para cada valor de $\epsilon$ a través de la pendiente de la gráfica del desplazamiento cuadrático medio para cada valor de $M$ en el intervalo temporal (100-400), dentro del cual es válida la relación de Einstein (6.1). Los resultados se muestran en la tabla 6.3; al igual que en las tablas 6.1 y 6.2 la incertidumbre es un orden de magnitud menor a la última cifra reportada.

Tabla 6.3: Coeficiente de difusión en el régimen anarmónico

\begin{tabular}{|c|cccc|}
\hline & \multicolumn{5}{c|}{$M$} & $\mathbf{8 0}$ & $\mathbf{1 0 0}$ \\
\hline$\epsilon$ & $\mathbf{4 0}$ & $\mathbf{6 0}$ & 0.0487 & 0.0506 \\
\hline 0.1 & 0.0499 & 0.049 & 0.0968 & 0.099 \\
\hline 0.2 & 0.113 & 0.1074 & 0.184 & 0.206 \\
\hline 0.4 & 0.208 & 0.212 & 0.263 & 0.287 \\
\hline 0.6 & 0.294 & 0.293 & 0.466 & 0.490 \\
\hline 1 & 0.50 & 0.519 & 1.051 & 0.884 \\
\hline 2 & 0.975 & 0.885 & 1.665 & 1.690 \\
\hline 4 & 1.80 & 1.81 & 2.68 & 2.19 \\
\hline 6 & 2.741 & 2.55 & 3.84 & 3.98 \\
\hline 10 & 4.01 & 4.08 & & \\
\hline
\end{tabular}

A continuación podemos estudiar el comportamiento de $D$ como función de 


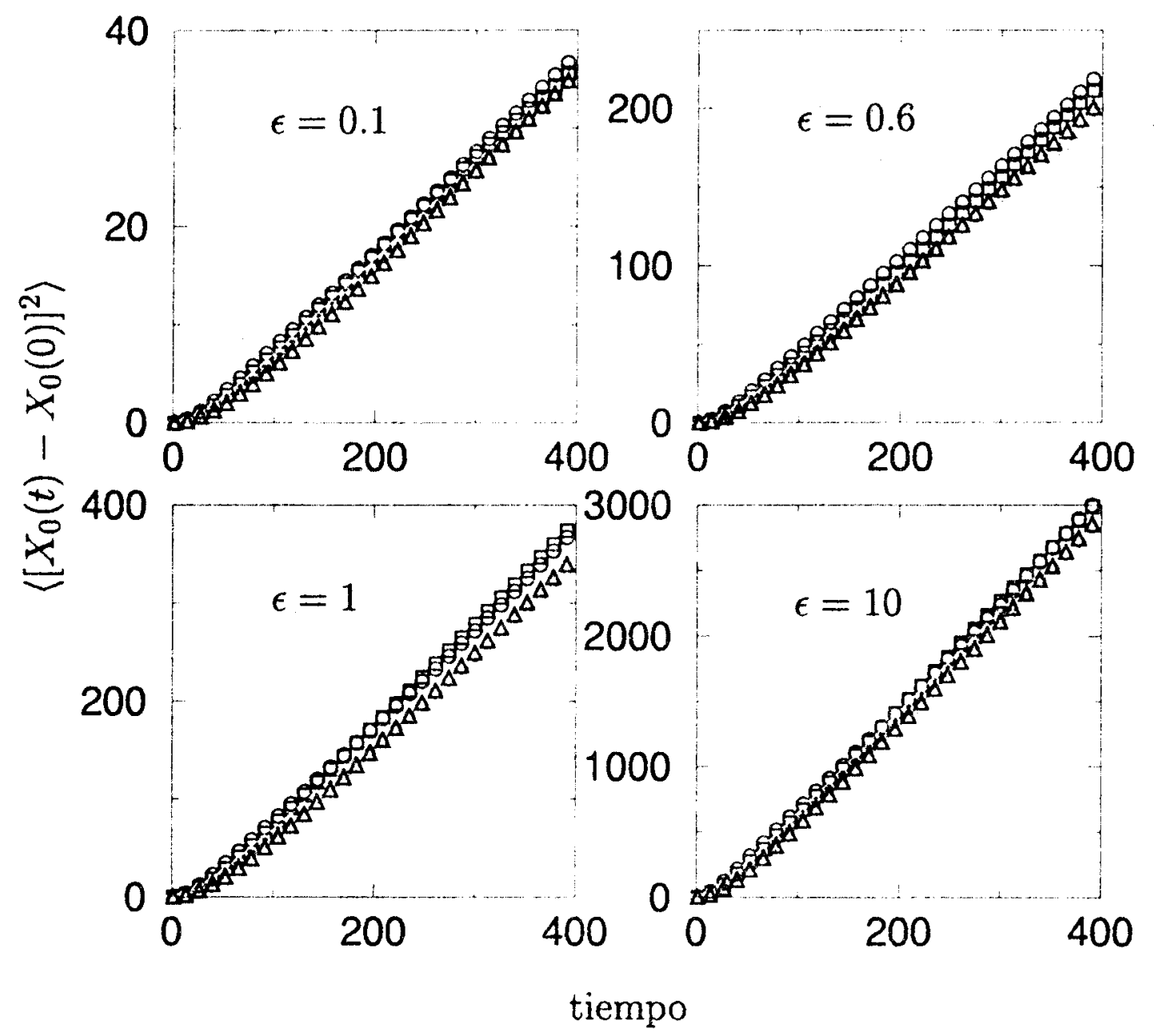

Figura 6.5: Desplazamiento cuadrático medio del oscilador pesado correspondiente a $M=40$ (círculos), 60 (cuadrados), 80 (diamantes) y 100 (triángulos) para distintos valores de $\epsilon$ con $N=300000$ en todos los casos.

є. Pero antes, y al igual que en el caso armónico, tenemos que examinar cómo dependen los distintos valores obtenidos de $D_{M}$ con respecto a la media aritmética $\bar{D}$, pero ahora para cada valor de $\epsilon$. De los valores en la tabla 6.3 vemos que, para una cierta $\epsilon$, los valores de $D_{M}$ para las distintas masas utilizadas no son tan uniformes como en el caso armónico. De hecho, podría inferirse de los datos que existe una dependencia débil del coeficiente de difusión con respecto a $M$. La cuestión importante es si esta dependencia es estadísticamente significativa. Examinando con mayor cuidado los valores de la tabla 6.3 encontramos que no 
existe una desviación sistemática que permita inferir una dependencia significativa del coeficiente de difusión respecto a $M$. De hecho, ningún valor de $D_{M}$, para una cierta $\epsilon$, tiene una desviación mayor a $7 \%$ de la media aritmética $\bar{D}$. Por lo tanto, podemos considerar que el coeficiente de difusión está dado por $D \approx \bar{D}$ para un valor fijo de $\epsilon$. Podemos entonces graficar a $D$ como función de $\epsilon$; los resultados obtenidos se reportan en la figura 6.6.

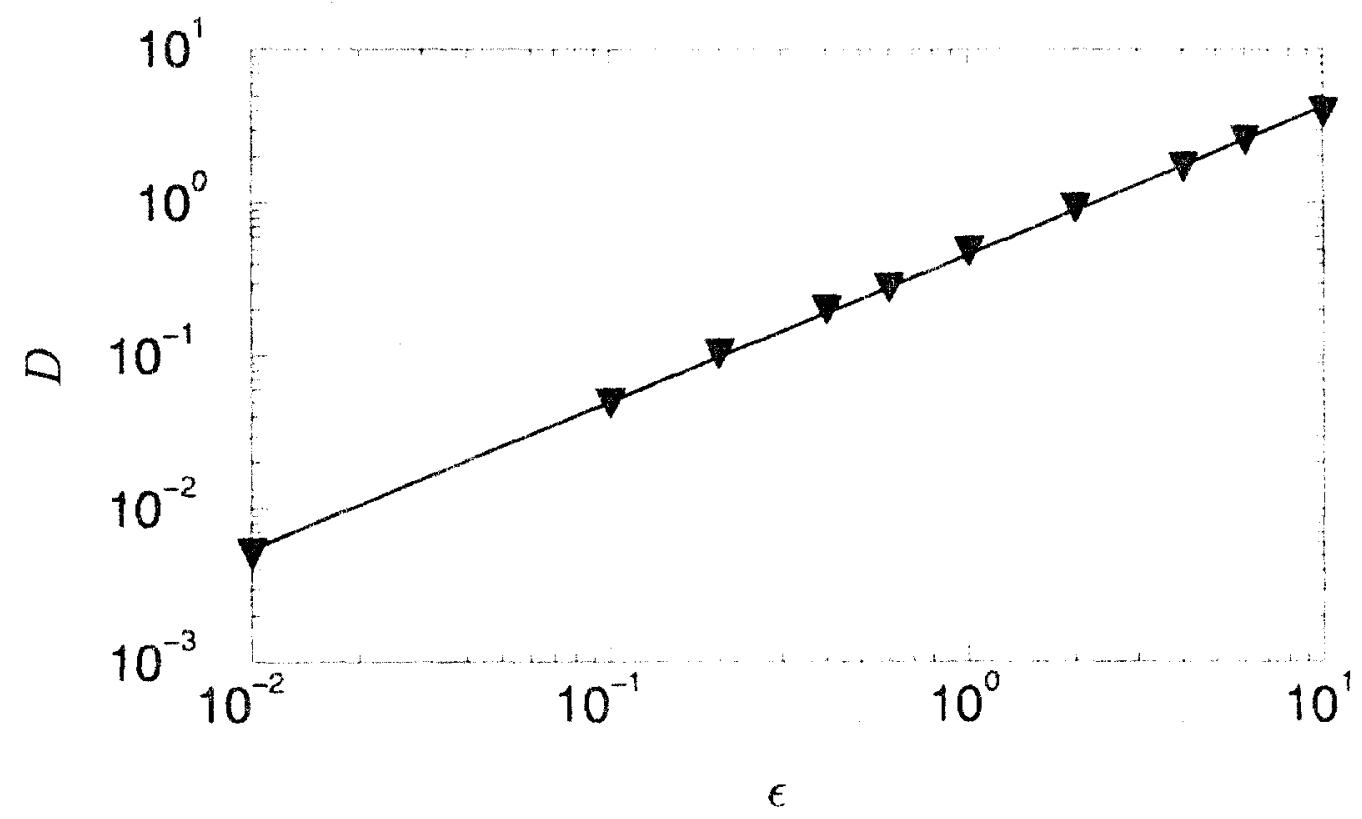

Figura 6.6: Dependencia del coeficiente de difusión $D$ con respecto a la densidad de energía $\epsilon$. La linea recta representa el mejor ajuste de mínimos cuadrados a los datos numéricos.

De la figura resulta que el coeficiente de difusión $D$ tiene una dependencia con respecto a la densidad de energía $\epsilon$ de la forma $D=D_{0} \epsilon^{m}$, donde $D_{0}=0.466 \pm 0.008$ y $m=0.964 \pm 0.008$. De la validez de este escalamiento para todo valor de $\epsilon$ (que incluye desde el régimen armónico hasta el caótico) concluimos que no hay evidencia de alguna característica de la dinámica microscópica (Hamiltoniana) en el comportamiento del coeficiente de difusión $D$ que pueda inferirse a partir de nuestros datos numéricos. Este resultado es congruente con aquellos obtenidos por Dettmann et al. [2, 3] para los modelos de Eherenfest (regular) y de Lorentz (caótico), ya que estos autores estudiaron el comportamiento difusivo de una partícula en un arreglo aleatorio de obstáculos, tanto cuadrados 
(modelo de Eherenfest) como circulares (modelo de Lorentz), y no encontraron diferencias cualitativas entre ambos modelos, aunque tenían una dinámica completamente diferente.

A continuación tenemos que dar una explicación de por qué no es posible observar alguna manifestación del caos microscópico en el comportamiento difusivo de nuestro modelo. Como se recordará del capítulo 2 , al estudiar la fenomenología del movimiento Browniano habíamos visto que el comportamiento difusivo descrito por la relación de Einstein (6.1) es válido para tiempos $t \gg \tau$, donde $\tau$ es el tiempo de correlación de la función de autocorrelación del momento $\rho_{0}(t)$. Este hecho es una consecuencia de que los cambios en la pasición de una partícula Browniana ocurren en una escala de tiempo que es grande en comparación con aquella en la que ocurren cambios significativos en el momento de la misma, como ya también mencionábamos en el capítulo 2 . Cuando $t \gg \tau$ ya se han perdido todos los detalles sobre las condiciones iniciales del sistema y sólo se puede obtener información asintótica de la posición del oscilador pesado a través del comportamiento asintótico del desplazamiento cuadrático medio. Por otra parte; para $t \ll \tau$ o incluso $t \approx \tau$ es posible estudiar propiedades del oscilador pesado que corresponden al espacio de momentos del sistema y que hasta este momento hemos ignorado. Ahora bien, estudiar el comportamiento del oscilador pesado en estos regímenes temporales es equivalente a estudiar el comportamiento de la función $\rho_{0}(t)$, lo que haremos a continuación.

\subsubsection{Tiempo de Relajación $\tau$}

De manera totalmente análoga al caso armónico podemos calcular la función $\rho_{0}(t)$ en el intervalo de valores de $\epsilon$ que corresponden al régimen anarmónico. Para algunos valores particulares de $\epsilon$ los resultados se pueden apreciar en las figuras 6.7 y 6.8. Es claro que el comportamiento de $\rho_{0}(t)$, en la escala temporal mostrada, es aproximadamente exponencial, de manera totalmente análoga al caso armónico ya antes estudiado. Por lo tanto, se puede inferir con un grado razonable de certeza que el oscilador pesado se comporta como una partícula Browniana. Para cada valor de $\epsilon$ el ajuste con la correspondiente aproximación exponencial $\rho_{0}(t)=\exp (-t / \tau)$ es bastante bueno, como se puede apreciar en las figuras en escala semilogarítmica 6.9 y 6.10 , que corresponden a las figuras 6.7 y 6.8 , respectivamente.

La propiedad que caracteriza a la función $\rho_{0}(t)$ es el tiempo de relajación $\tau$, 


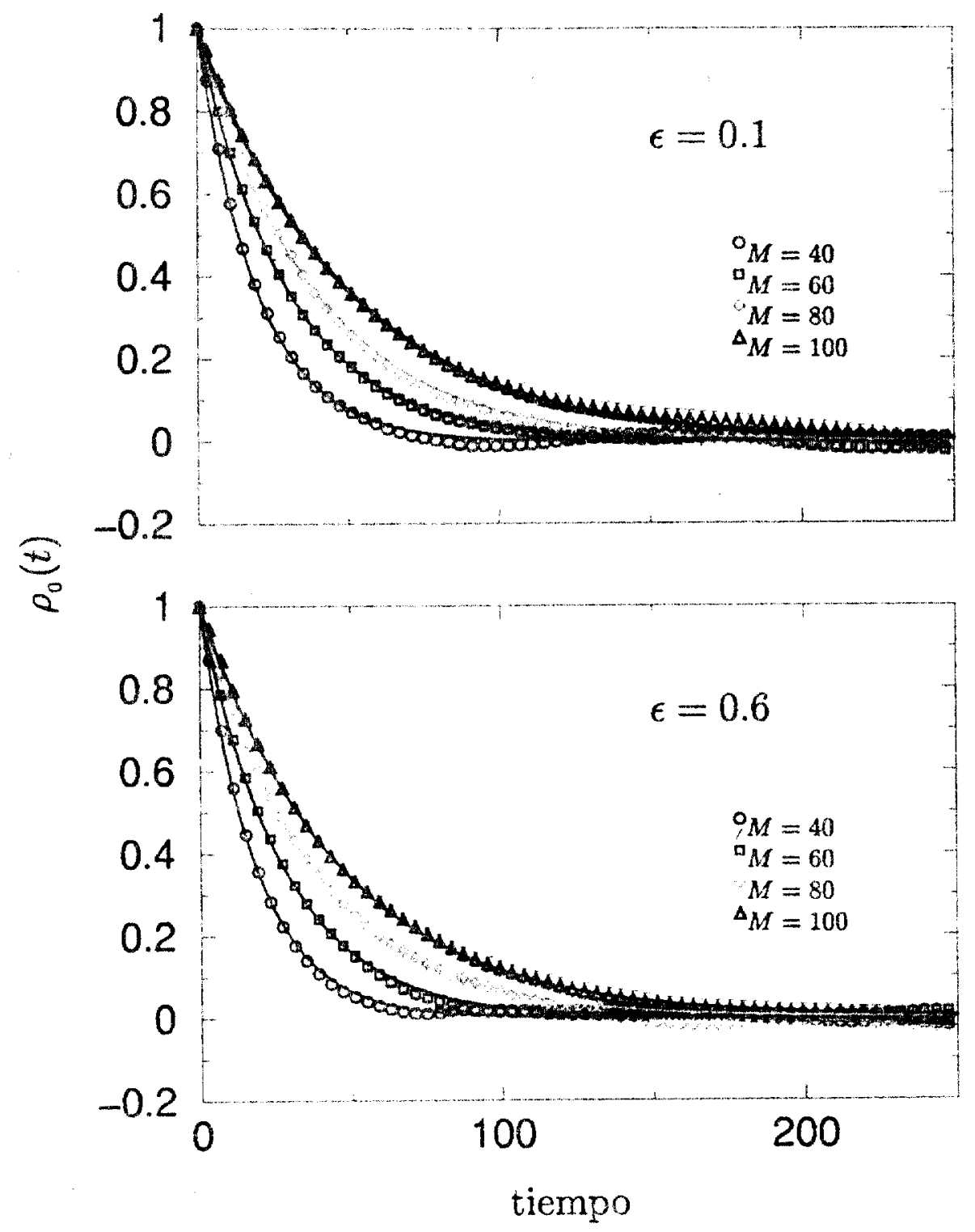

Figura 6.7: Función de autocorrelación $\rho_{0}(t)$ del oscilador pesado para distintos valores de $\varepsilon$ con $N=300000$ y todos los valores de $M$ utilizados. Las lineas continuas para cada valor de $M$ corresponden al ajuste $\exp (-t / \tau)$, donde $\tau$ se calcula a partir de la expresión (6.6).

el cual podemos calcular a partir de (6.6). Para una $\epsilon$ y una $M$ dadas tenemos ahora que definir un tiempo máximo para el límite superior de (6.6). Comparando 


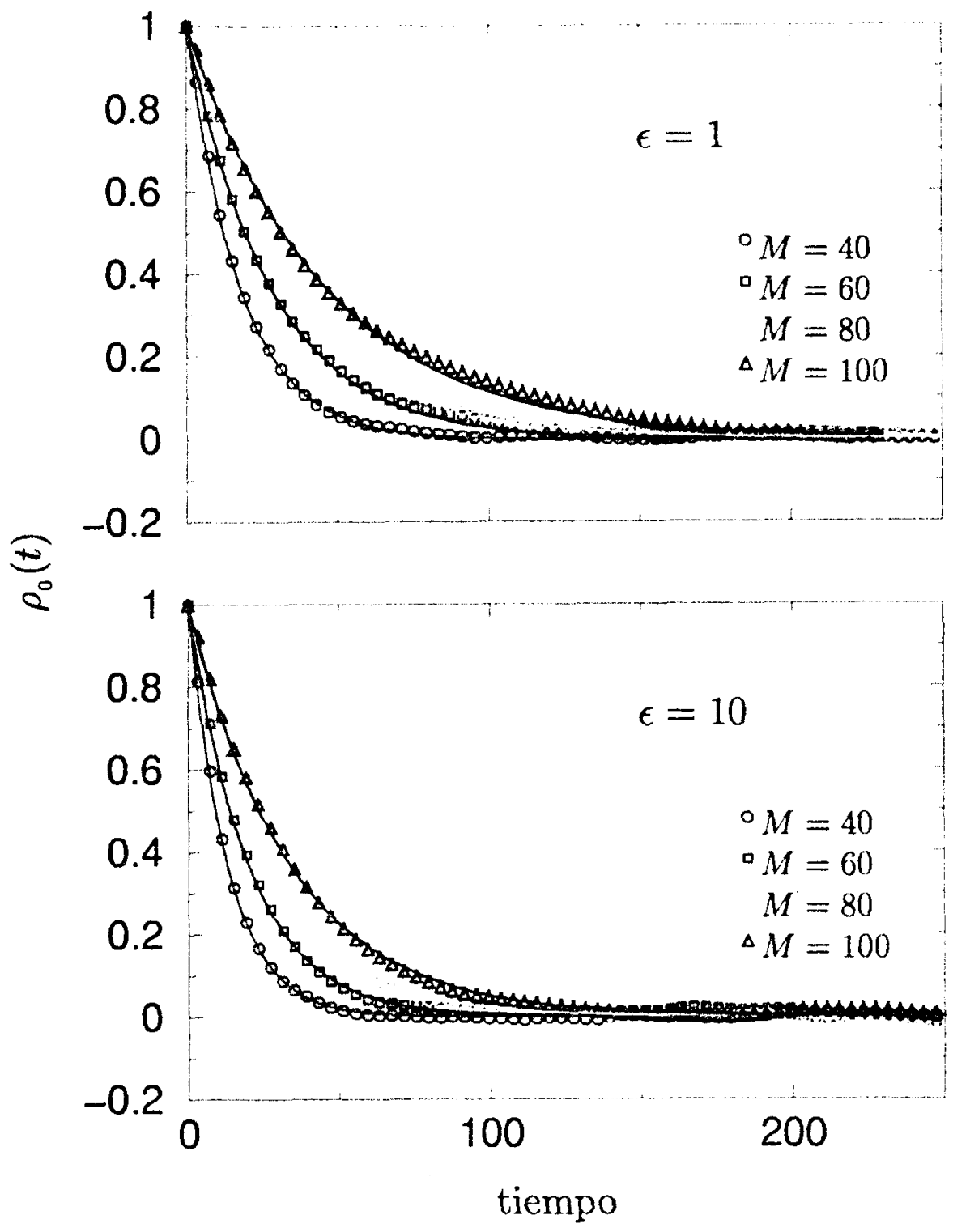

Figura 6.8: Resultados análogos a los de la figura 6.7, pero para otros valores de $\epsilon$.

con el caso armónico tenemos que el efecto de tomar un valor de $\epsilon$ en el régimen caótico es el de disminuir el valor de $\tau$ en todos los casos considerados. Entonces podemos tomar como límite superior de la integral (6.6) a la cota superior de la desigualdad (6.4), tal y como se hizo en el caso armónico. Para comprobar la validez de los valores de $\tau$ calculados a partir de (6.6) podemos calcular los 


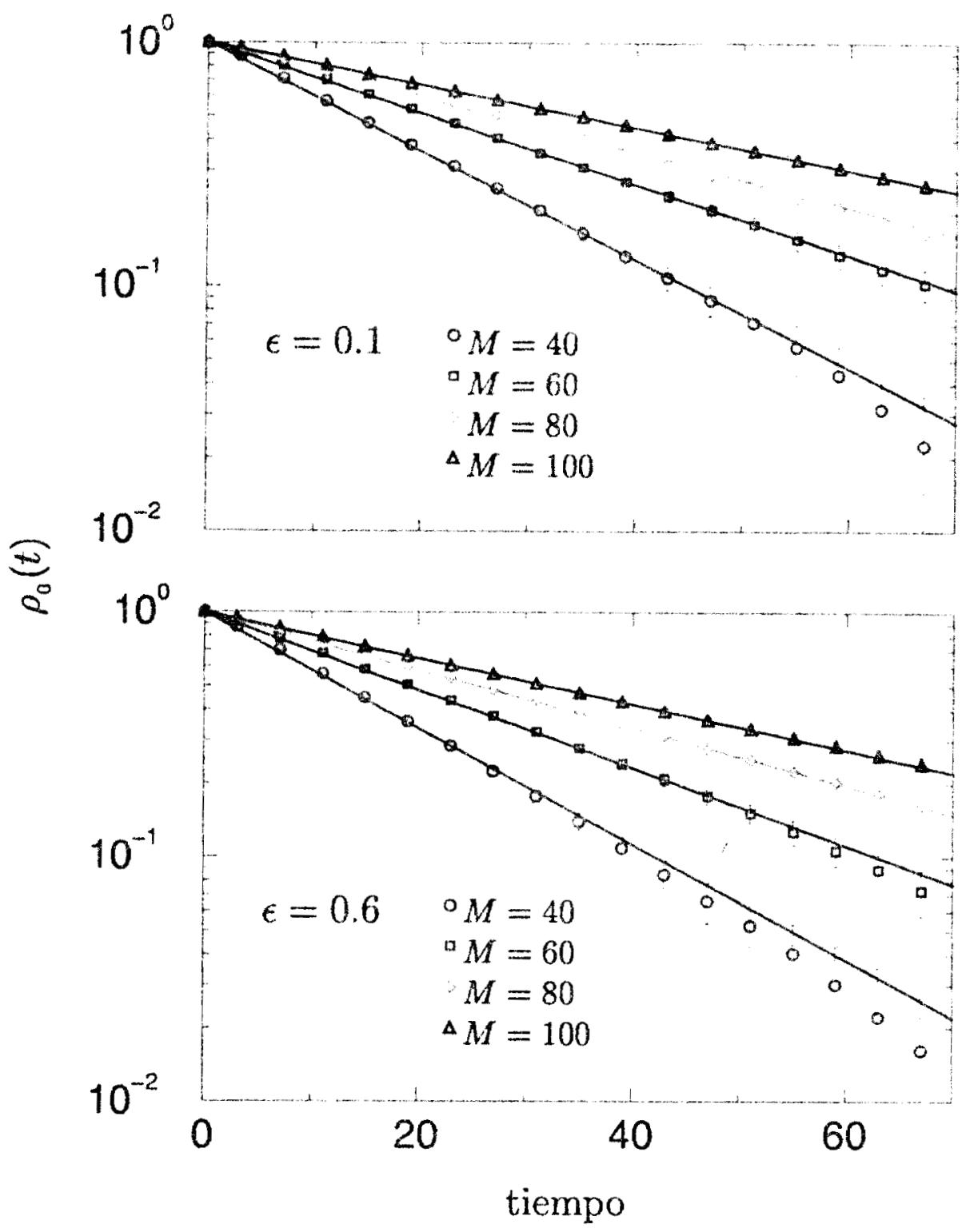

Figura 6.9: Logaritmo de la función de autocorrelación normalizada $\rho_{0}(t)$ del oscilador pesado para distintos valores de $\epsilon$ con $N=300000$ y todos los valores de $M$ utilizados. Las lineas continuas para cada valor de $M$ corresponden al ajuste $\exp (-t / \tau)$, donde $\tau$ se calcula a partir de la expresión (6.6).

tiempos $\tau$ a partir de cada una de las pendientes de las rectas definidas por los puntos de simulación en las figuras 6.9 y 6.10. La diferencia con respecto al valor 


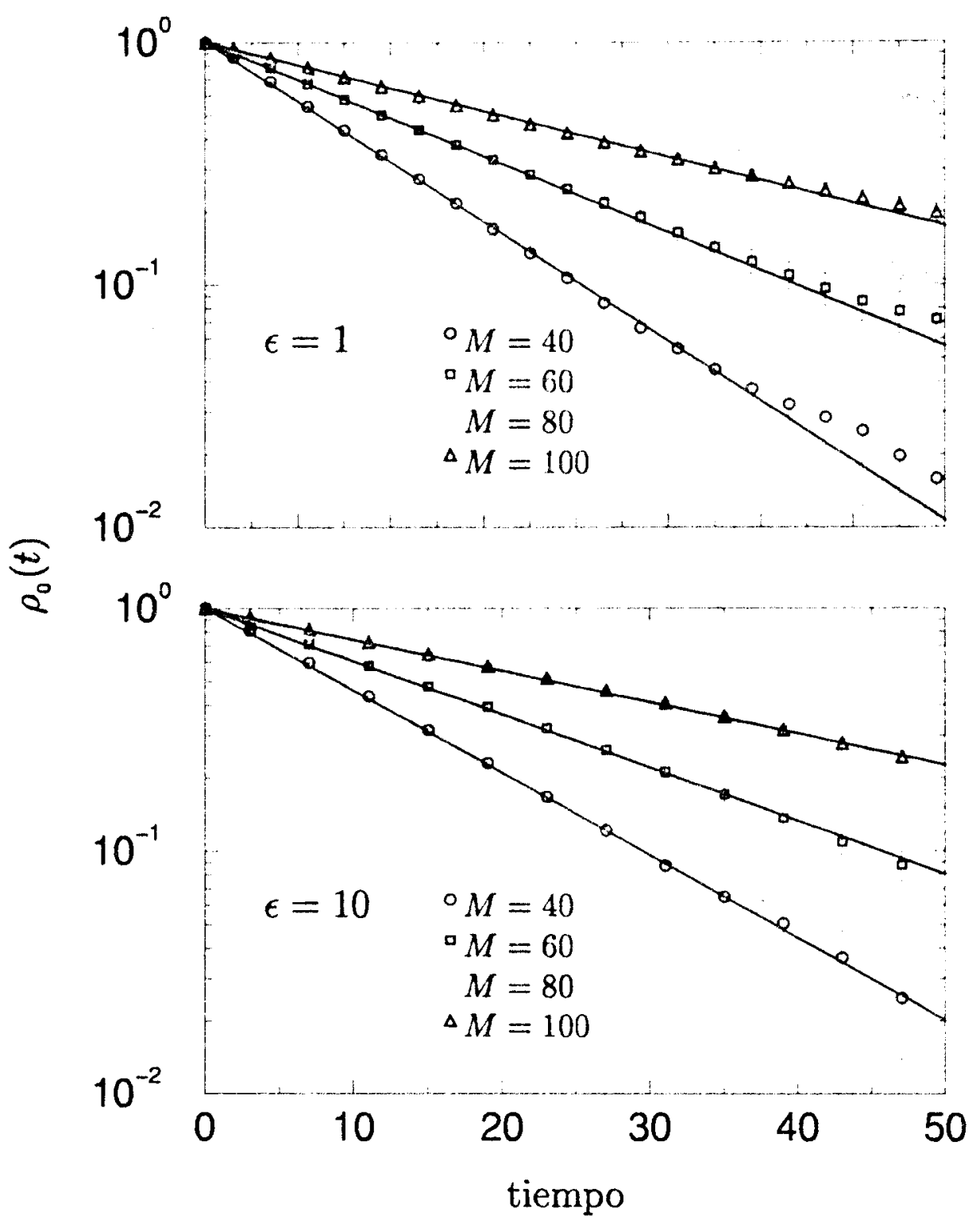

Figura 6.10: Resultados análogos a los de la figura 6.9, pero para otros valores de $\epsilon$.

obtenido utilizando (6.6) no es estadisticamente significativa, por lo que el procedimiento originalmente utilizado es perfectamente válido. Ahora bien, calculando $\tau$ de cualquiera de las maneras antes mencionadas podemos obtener una serie de valores de $\tau$ para una $M$ fija y cada $\epsilon$ considerada, repitiendo posteriormente el procedimiento para todos los valores de $M$ utilizados. El resultado se muestra en 
la figura 6.11 .

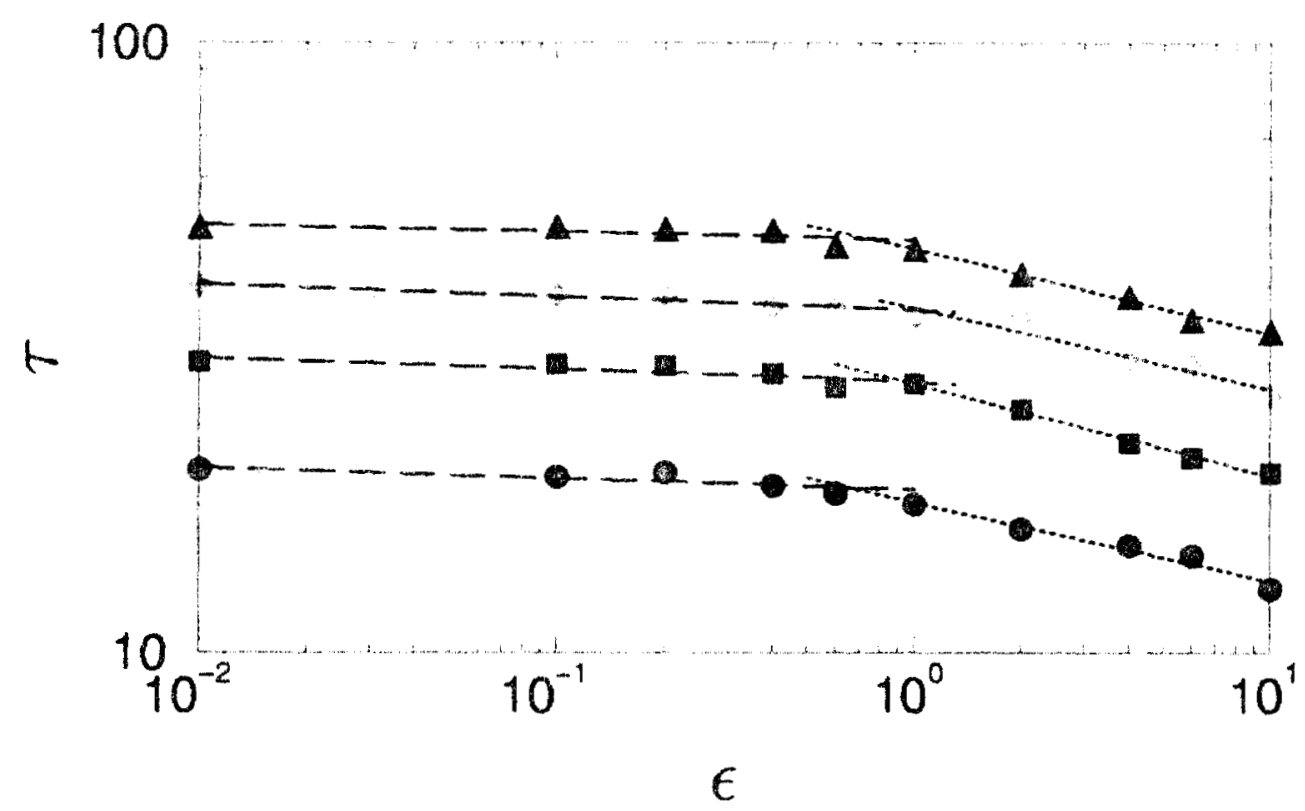

Figura 6.11: Tiempo de relajación $\tau$ vs densidad de energía $\epsilon$. Los símbolos corresponden a $M=40$ (círculos), 60 (cuadrados), 80 (diamantes) y 100 (triángulos). Las lineas rectas representan los ajustes de mínimos cuadrados en las regiones $\epsilon<1$ y $\epsilon \geq 1$.

Puede apreciarse, en la escala log-log utilizada, que los puntos de simulación están separados en dos regiones diferentes y bien definidas, dependiendo del valor de $\epsilon$. Cuando $\epsilon<1$, la dependencia de $\tau$ con respecto a la densidad de energía es bastante débil. Por el contrario, cuando $\epsilon \geq 1, \tau$ decrece de manera muy notoria conforme aumenta el valor de $\epsilon$. En ámbos regínenes la dependencia funcional de $\tau$ con respecto a $\epsilon$ es de la forma

$$
\tau_{M}(\epsilon)= \begin{cases}\tau_{0, M} \epsilon^{\alpha_{M}}, & 0.01 \leq \epsilon<1 \\ \widetilde{\tau}_{0, M} \epsilon^{\sigma_{M}}, & 1 \leq \epsilon \leq 10 .\end{cases}
$$

De la figura 6.11 resulta claro que, para todos los datos de simulación correspondientes a una cierta $M$ tenemos que $\tau_{0, M} \neq \widetilde{\tau}_{0, M}$ y $\alpha_{M} \neq \widetilde{\alpha}_{M}$; esto es, las constantes de escalamiento son diferentes en el régimen armónico $(\epsilon<1)$ y caótico $(\epsilon \geq 1)$. Este resultado contrasta de manera notoria con el reportado en la figura 6.6; en 
ésta, la dependencia $D=D_{0} \epsilon^{m}$ era válida para todo el intervalo de valores de $\epsilon$, desde el régimen armónico hasta el caótico. El comportamiento de $\tau$ muestra, por el contrario, un cambio conforme se pasa del régimen armónico al caótico. Ahora bien, aunque la relación (6.9) fue obtenida de manera numérica (ajuste de mínimos cuadrados) y no existen aproximaciones analiticas en el régirnen caótico que permitiesen una comprobación independiente, su plausibilidad es bastante grande ya que, en el régimen armónico, la validez de (6.9) se puede comprobar observando que los datos de simulación para $\epsilon=0.01$ coinciden, con una precisión mejor que el $1 \%$, con el resultado exacto $\tau=M / 2$ para todos los valores de $M$.

Ahora bien, de los escalamientos presentados en la figura 6.11 se tiene que $\alpha_{M} \approx \alpha_{M^{\prime}}$ para toda $M \neq M^{\prime}$ en el régimen $\epsilon<1$. Tambien tenemos que $\tilde{\alpha}_{M} \approx \tilde{\alpha}_{M^{\prime}}$ para $M \neq M^{\prime}$ y $\epsilon \geq 1$. Estos hechos implican que los exponentes en el escalmiento (6.9) para cada régimen tienen aproximadamente el mismo valor, independientemente del valor de $M$ al que correspondan. Por lo tanto es razonable suponer la existencia de exponentes de escalamiento $\alpha^{*}$ y $\tilde{\alpha}^{*}$ independientes de la masa del oscilador pesado. Para calcular $\alpha^{*} \cdot\left(\tilde{\alpha}^{*}\right)$ rescalarnos los datos de la figura 6.11 en el régimen $\epsilon<1(\epsilon \geq 1)$ con sus correspondientes valores de $\tau_{0, M}$ $\left(\tilde{\tau}_{0, M}\right)$. Definimos entonces las variables

$$
\begin{array}{rlrl}
\epsilon^{*} & \equiv \ln \epsilon / \ln \tau_{0, M} & & \left(\epsilon^{*} \equiv \ln \epsilon / \ln \widetilde{\tau}_{0, M}\right) \\
\tau^{*} & \equiv \ln \tau / \ln \tau_{0, M} & \left(\tau^{*} \equiv \ln \tau / \ln \widetilde{\tau}_{0, M}\right) .
\end{array}
$$

El resultado lo podemos apreciar en la figura 6.12.

Como puede verse hemos obtenido un escalamiento común para todos los datos de simulación en cada uno de los regímenes $\epsilon<1$ y $\epsilon \geq 1$. A partir de los correspondientes ajustes por mínimos cuadrados es inmediato calcular los exponentes de escalamiento $\alpha^{*}$ y $\tilde{\alpha}^{*}$. Tenemos entonces que la forma explícita del escalamiento de $\tau^{*}$ con respecto a $\epsilon^{*}$ en cada uno de los regímenes estudiados puede escribirse de la forma

$$
\tau^{*}\left(\epsilon^{*}\right) \sim\left\{\begin{array}{l}
\left(\epsilon^{*}\right)^{-0.017 \pm 0.004}, \quad-1.57541 \leq \epsilon^{*}<\epsilon_{c}^{*} \\
\left(\epsilon^{*}\right)^{-0.136 \pm 0.007}, \quad \epsilon_{c}^{*}>\epsilon^{*} \leq 0.80121 .
\end{array}\right.
$$

donde $\epsilon_{c}^{*} \approx 0$ y que corresponde al punto en el que ocurre el cambio de escalamiento. Este último es muy pronunciado, ya que los exponentes obtenidos difieren en un orden de magnitud. Tenemos entonces que, en el cambio de escalamiento de $\tau^{*}$ se está manifestando la transición del comportamiento del sistema de armónico a caótico. Este cambio ocurre para un valor específico de $\epsilon_{c}^{*}$. 


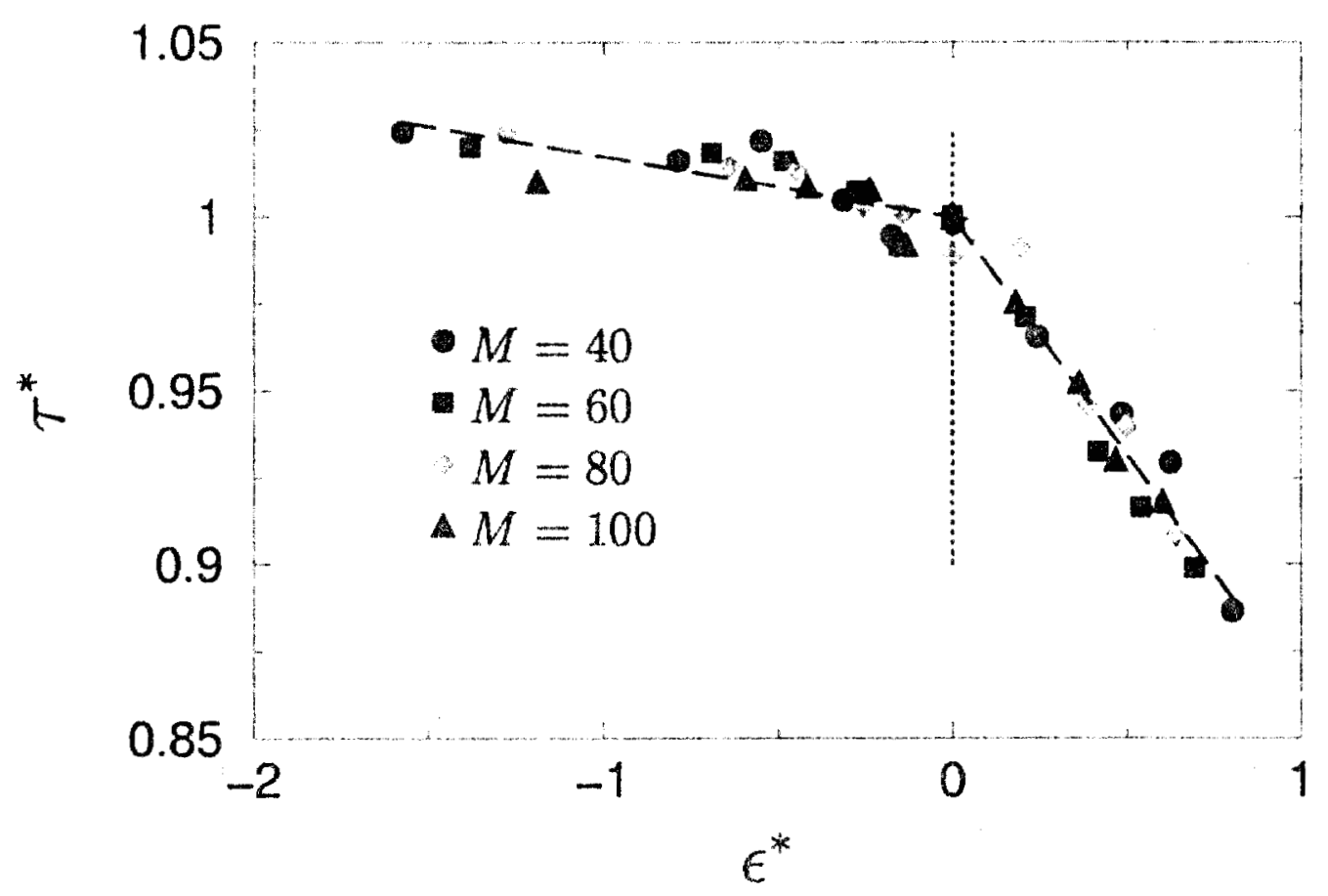

Figura 6.12: Mismos datos que en la figura 6.11, pero en términos de la variables rescaladas $\tau^{*}$ y $\epsilon^{*}$. Las lineas rectas representan los ajustes de mínimos cuadrados en las regiones $\epsilon^{*}<0$ y $\epsilon^{*} \geq 0$.

\subsubsection{Discusión}

La primera observación que podemos hacer sobre los resultados antes presentados concierne a la relación entre $\tau$ y $D$ que puede obtenerse a través de la fórmula de Green-Kubo (2.34), la cual se puede escribir como

$$
D_{M}=\frac{T}{M} \int_{0}^{\infty} \rho_{0}(t) d t
$$

utilizando el principio de equipartición $\left\langle P_{0}^{t}(0)\right\rangle=M T$. Si en esta última expresión sustituimos la forma exponencial $\rho_{0}(t)=\exp (-t / \tau)$ obtenemos

$$
D_{M}=\left(\frac{T}{M}\right) \tau
$$


Esta relación muestra que $D$ y $\tau$ no son variables independientes, por lo que el cambio de escalamiento de $\tau$ como función de $\epsilon$ mostrado en la figura 6.11 debería de poder observarse en la dependencia de $D$ respecto a $\epsilon$ mostrada en la figura 6.6, pero no ocurre así. La elucidación de esta aparente contradicción es muy importante porque, de la validez del escalamiento $D=D_{0} \epsilon^{m}$ en todo el intervalo de valores de $\epsilon$, concluimos que no había evidencia de caos microscópico en el comportamiento difusivo del oscilador pesado.

Para resolver este problema primeramente observamos que $T / M \ll 1$ para todos los valores de $M$ considerados. Esto implica que los detalles del comportamiento de $\tau$ se pierden cuando se multiplica este tiempo de relajación por el factor $T / M$. Para hacer mas explícita esta explicación mostramos, en la tabla 6.4, el cálculo de $D_{M}$ a partir de $\tau$ para el caso particular $M=40$; un análisis semejante para los demás valores de $M$ permite hacer observaciones similares.

Tabla 6.4: Valores de $D_{M}$ a partir de $\tau$. Caso particular $M=40$

\begin{tabular}{|l|cccc|}
\hline$\epsilon$ & $\tau$ & $T$ & $T / M$ & $(T / M) \tau$ \\
\hline 0.1 & 19.4891 & 0.1007694 & 0.0025192 & 0.040969 \\
\hline 0.2 & 19.8165 & 0.2028052 & 0.0050701 & 0.100472 \\
\hline 0.4 & 18.8570 & 0.4100227 & 0.0102505 & 0.193294 \\
\hline 0.6 & 18.2922 & 0.6206903 & 0.0155172 & 0.283844 \\
\hline 1 & 17.6088 & 1.0500724 & 0.0262518 & 0.462263 \\
\hline 2 & 16.0323 & 2.1560271 & 0.0539006 & 0.864151 \\
\hline 4 & 15.0357 & 4.4493466 & 0.1112336 & 1.672475 \\
\hline 6 & 14.4557 & 6.8046176 & 0.1701154 & 2.459137 \\
\hline 10 & 12.7883 & 11.6182387 & 0.2904559 & 3.714437 \\
\hline
\end{tabular}

Como podemos claramente observar, los valores de la última columna en la tabla 6.4 son muy semejantes a los correspondientes valores de la primera columna de la tabla 6.3. Un análisis semejante puede hacerse para los restantes valores de $M$ y así construir una tabla semejante a 6.3. Si se toma el promedio aritmético $\bar{D}$ de todos los valores $D_{M}$ para cada valor de $\epsilon$, entonces se obtiene un conjunto de datos que, al graficarlos, reproducen los resultados de la figura 6.6. Deducimos que no hay contradicción, pues, aunque el cambio de comportamiento respecto a $\epsilon$ existe en el coeficiente de difusión $D$ en virtud de la relación (6.14), este cambio es inobservable debido al orden de magnitud del factor $T / M$ en todos los casos considerados.

A continuación debemos estudiar el origen del cambio de escalamiento en $\tau$ 
observado en la figura 6.11. Debido a la simplicidad de nuestro sistema la hipótesis más probable es que cl cambio de escalamiento tenga su origen en la dinámica microscópica del sisterna. En el capitulo 5 habiamos visto que la distancia $d(t)$ entre dos trayectorias cuyos puntos iniciales están separados por una distancia infinitesimal proporciona información adecuada sobre el comportamiento dinámico del modelo conforme cambia el valor de $\epsilon$. En la figura 6.13 presentamos un caso particular con $M=60$ y $N=300000$; para otros valores de $M$ los resultados son análogos.

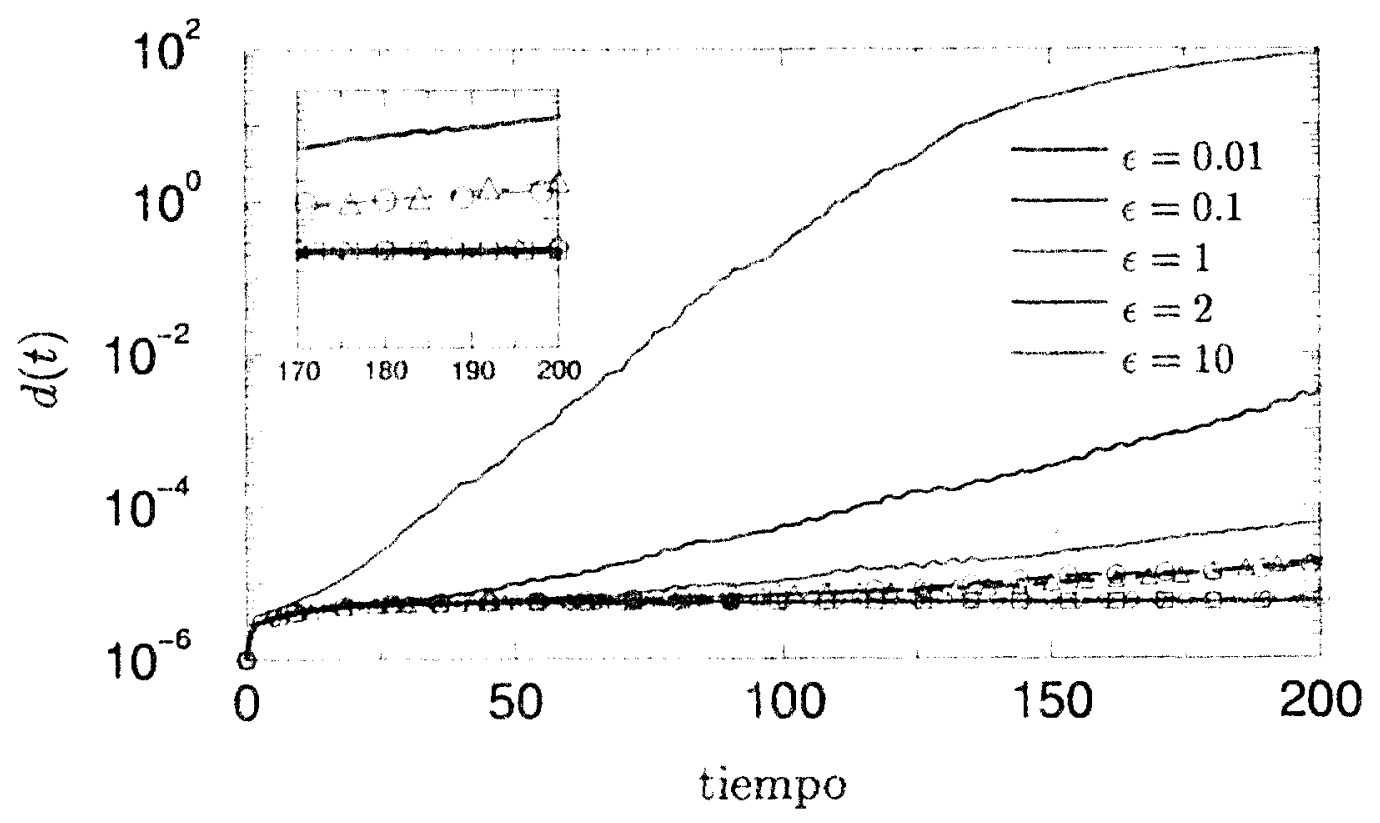

Figura 6.13: Distancia en el espacio fase entre pares de trayectorias para el caso $M=60$ y $N=300000$. Las curvas en linea discontinua entre $\epsilon=0.1$ (rojo) y $\epsilon=1$ (verde) corresponden a los casos $\epsilon=0.2$ (cuadrados), 0.4 (diamantes), 0.6 (círculos) y 0.8 (triángulos). En la figura pequeña se muestra con más detalle el comportarniento asintótico de $d(t)$.

Como puede apreciarse, esta figura es prácticamente indistinguible de 5.2 para el mismo valor de $M$, aunque $N$ difiera en ambas figuras en dos órdenes de magnitud. El comportaiento dinámico es entonces independiente del número $N$ de osciladores livianos, al menos según la información proporcionada por la distancia 
$d(t)$. Hemos además calculado este parámetro en el intervalo $0.1<\epsilon<1$. Para $\epsilon=0.2$ y 0.4 la distancia $d(t)$ se mantiene dentro del mismo orden de magnitud, al igual que para los casos $\epsilon=0.01$ y $\epsilon=0.1$ también mostrados. Para $\epsilon=0.6$ y 0.8 la distancia empieza a tener un comportamiento exponencialmente divergente muy débil; sólo para $\epsilon=1$ podemos considerar que el cambio de magnitud en $d(t)$ es de más de un orden de magnitud, lo que es característico de una dinámica caótica. Por lo tanto podemos ver que la transición dinámica entre el comportamiento armónico y caótico ocurre para valores de $\epsilon \approx 1$. Esta "frontera" entre ambos regímenes es difusa, pero es lo suficientemente definida como para poder afirmar que, en el intervalo $\epsilon<1$, el comportamiento de nuestro sistema es prácticamente indistinguible del comportamiento armónico reproducido para el valor particular $\epsilon=0.01$. Por otra parte, en el intervalo $\epsilon \geq 1$ la dinámica microscópica es caótica, con $\epsilon=10$ definiendo el línite superior de un régimen de caos plenamente desarrollado (la distancia $d(t)$ presenta la máxima divergencia posible consistente con una elección particular de las condiciones iniciales). Estos rangos de valores de $\epsilon$ coinciden de manera muy cercana con aquellos obtenidos de la figura 6.11 para la dependencia de $\tau$ respecto a $\epsilon$. De esta similitud podemos concluir que el cambio de escalamiento en $\tau$ es debido al cambio en la dinámica microscópica del modelo cuando $\epsilon \approx 1$. Es importante resaltar que $\tau$ es un parámetro asociado exclusivamente al oscilador pesado, por lo que hemos confirmado que este último se comporta efectivamente como una sonda del comportamiento dinámico del sistema de muchos grados de libertad al que está acoplado.

Concluyamos este capítulo con dos observaciones adicionales. La transición entre el régimen armónico y el caótico ha sido también caracterizada estudiando numéricamente el comportamiento del tiempo de decaimiento $\tau_{R}$ de la entropía espectral (4.7) con respecto a $\epsilon$. Para el modelo FPU- $\beta$ [67], así como para otros modelos unidimensionales [124], se tiene que $\tau_{R}$ es constante para valores altos de $\epsilon$, pero se incrementa significativamente debajo de cierto umbral $\epsilon_{R}$. Para el modelo FPU- $\beta$ se tiene que $\epsilon_{n} \approx 1$; este umbral coincide aproximadamente con el umbral $\epsilon \approx 1$ que existe entre las regiones armónica y caótica de nuestro modelo y que se obtiene de analizar la dinámica a partir de la figura 6.13 y también del análisis del comportamiento del tiempo de relajación $\tau$ de $\rho_{0}(t)$ presente en la figura 6.11. Este mismo cambio de comportamiento del tiempo de decaimiento $\tau_{R}$ de una forma modificada de la entropía espectral (4.7) también ha sido estudiado numéricamente para el caso de la relajación hacia el equilibrio de un modelo de Xe sólido con impurezas de $\mathrm{I}_{2}[61]$. En ese trabajo se arguye que los resultados obtenidos por 
medio de la entropía espectral son semejantes a aquellos obtenidos estudiando el tiempo de relajación de la función de autocorrelación de la velocidad de un átomo de la molécula de Yodo, por lo que la transición entre el régimen armónico y caótico puede, en principio, estudiarse experimentalmente. Ahora bien, aunque nuestro modelo es muy simplificado y no corresponde exactamente a una situación experimental real, la metodología que hemos utilizado para estudiarlo puede tener mayores ventajas que aquella expuesta en [61], ya que la función $\rho_{0}(t)$ la hemos estado calculando en el estado de equilibrio, mientras que, a través de la entropía espectral se estudia la relajación del sistema hacia el equilibrio partiendo de una configuración artificialmente impuesta al sistema.

Por último, recordemos que el efecto del defecto pesado sobre la dinámica del resto de los osciladores livianos que componen al sistema es despreciable; ambos están sólo débilmente acoplados. Sin embargo es posible obtener información sobre la dinámica de la cadena estudiando únicamente el comportamiento del oscilador pesado. Por lo tanto, este último juega el papel de una sonda del comportamiento global del sistema, coroo ya lo habíamos notado anteriormente. Pero en la literatura han sido estudiados otros tipos de sistemas reticulares en los cuales un defecto en la red produce un efecto totalmente diferente al de nuestro oscilador pesado. Estos sistemas consisten, esencialmente, en un conjunto de péndulos caóticos acoplados, teniendo uno de ellos una longitud menor que los demás. El resultado es que este único péndulo es capaz de producir patrones espacioternporalmente ordenados en lugar de un comportamiento cático complejo, tanto en una cadena lineal [125] de $N=128$ péndulos como en un arreglo bidimensional de tamaño $50 \times 50$ [126]. Por lo tanto, la introducción de este defecto proporciona un mecanismo para controlar el comportamiento dinámico de estos sistemas. Este efecto es totalmente opuesto al que nosotros hemos encontrado al perturbar la cadena FPU con un defecto pesado, por lo que nuestros resultados pueden iniciar una linea de investigación totalmente diferente, pero complementaria, a lo que hasta ahora se ha venido trabajando con respecto a sistemas dinámicos cáticos de muchos grados de libertad. 


\section{Capítulo 7}

\section{Conclusiones y Perspectivas}

En este trabajo hemos planteado un modelo unidimensional de osciladores anarmónicus que corresponde, esencialmente, a una extensión del modelo de osciladores armónicos acoplados a primeros vecinos utilizado con anterioridad para estudiar algunos aspectos del movimiento Browniano. Con la ayuda de este nuevo modelo hemos podido investigar un aspecto del movimiento Browniano hasta ahora muy poco estudiado: el efecto de la dinámica microscópica de la cadena de osciliadores sobre las propiedades macroscópicas del oscilador pesado. Los factores que nos permitieron obtener resultados en esta dirección fueron la sencillez del modelo y el hecho de que la cadena de osciladores livianos corresponde esencialmente al modelo FPU- $\beta$, extensamente estudiado en la literatura dentro del contexto de la equipartición de la energía y la relajación hacia el equilibrio. El hecho de que este modelo presente una transición muy bien definida entre dos regímenes dinámicos distintos nos permitió interpretar los resultados obtenidos como provenientes de la dinámica microscópica del modelo. Además, pudimos comprobar que las propiedades difusivas de este modelo, así como de otros ya estudiados anteriormente, no son las adecuadas para obtener información sobre la dinámica microscópica.

Existen varias lineas de investigación con las que se pueden ampliar los resultados del presente trabajo. La más inmediata consiste en investigar con más detalle la relación entre el escalamiento que hemos descubierto en el tiempo de relajación $\tau$ y la dinámica microscópica del modelo FPU- $\beta$. Aunque la relación entre el cambio de escalamiento en $\tau$ mostrado en la figura 6.11 y la distancia $d(t)$ (para $M=60$ en la figura 6.13) establece una conección bastante firme entre la dinámica Hamiltoniana de nuestro modelo y su comportamiento estadístico, es 
posible hacer un estudio mucho más detallado, ya que el hecho de que el cambio de escalamiento en $\tau$ ocurra de manera muy pronunciada para un rango de valores de $\epsilon$ muy específico puede estar relacionado a alguna característica particular de la dinámica Hamiltoniana del modelo FPU- $\beta$. Como ya lo habíanos mencionado en el capítulo 4, para este modelo se sabe que el máximo exponente de Lyapunov $\lambda_{1}$ presenta una dependencia con respecto a $\epsilon$ de la forma $\lambda_{1} \sim \epsilon^{\alpha}$. El exponente $\alpha$ no es constante para todo el rango de valores de $\epsilon$, sino que cambia de valor para el valor crítico $\epsilon_{\mathfrak{c}} \approx 1$, como se muestra en la figura 7.1. Este cambio de escalamiento tiene semajanza con el que se observa en la figura 6.11 para $\tau$ vs. $\epsilon$. Una extensión muy directa de nuestro trabajo consistiría en calcular $\lambda_{1}$ como función de $\epsilon$ y $M$ para nuestro modelo FPU modificado; posteriormente podría estudiarse si el valor crítico $\epsilon_{c}$ de la densidad de energía tiene efectivamente una dependencia con respecto a $M$ o permanece inalterado ( $\mathrm{y}$, por lo tanto, idéntico al valor $\epsilon_{c} \approx 0.8$ ya reportado en la literatura [115]).

Otra linea de investigación consiste en continuar el estudio sobre la equilibración del sistema que inciamos en el capítulo 5 . El resultado más firme que hemos obtenido es que el ritmo de termalización depende crucialmente del número de osciladores livianos $N$ y, en menor medida, de la masa $M$ del oscilador pesado. La principal dificultad en este sentido consiste en que hernos sido incapaces, hasta este momento, de identificar una función, análoga a la entropía espectral (4.7), que defina, de manera unívoca, qué tan alejado está el sistema del equilibrio. Una vez que pueda identificarse una furción semejante a $\mathcal{S}(t)$ (ver capítulo 4) para nuestro caso será posible definir un tiempo de equilibración $\tau_{e q}$ (que no debe de confundirse con el tiempo de relajación $\tau$ estudiado en el capítulo 6) y estudiar su dependencia con respecto a $\epsilon$ y $M$. La utilidad de esta linea de investigación estriba en que, como ya se mencionó en el capítulo 4, mucho esfuerzo se ha dedicado a estudiar la equilibración del modelo FPU (y otros similares), principalmente su dependencia respecto a $N$. Podemos aventurar la hipótesis de que, incluso cuando $\epsilon \gg 1$, subsisten regiones en el espacio fase del sistema que no son compatibles con el estado de equilibrio termodinámico y cuya medida disminuye a cero sólo cuando $N \gg 1$, que ha sido nuestro caso. De hecho, pareciera ser que el comportamiento no-estadístico de $\left\langle K_{p_{B}}\right\rangle_{t}$, claramente observable en las figuras 5.3 y 5.5 , es una manifestación de la existencia de las regiones ya antes aludidas y que sólo pudo ser puesto de manifiesto por la presencia del defecto pesado, ya que $\left\langle K_{B T}\right\rangle_{t}$ se comporta de la manera esperada y el valor de $T_{c i n}$ es consistente con el correspondiente valor obtenido para el caso de una cadena FPU de masas iguales. 


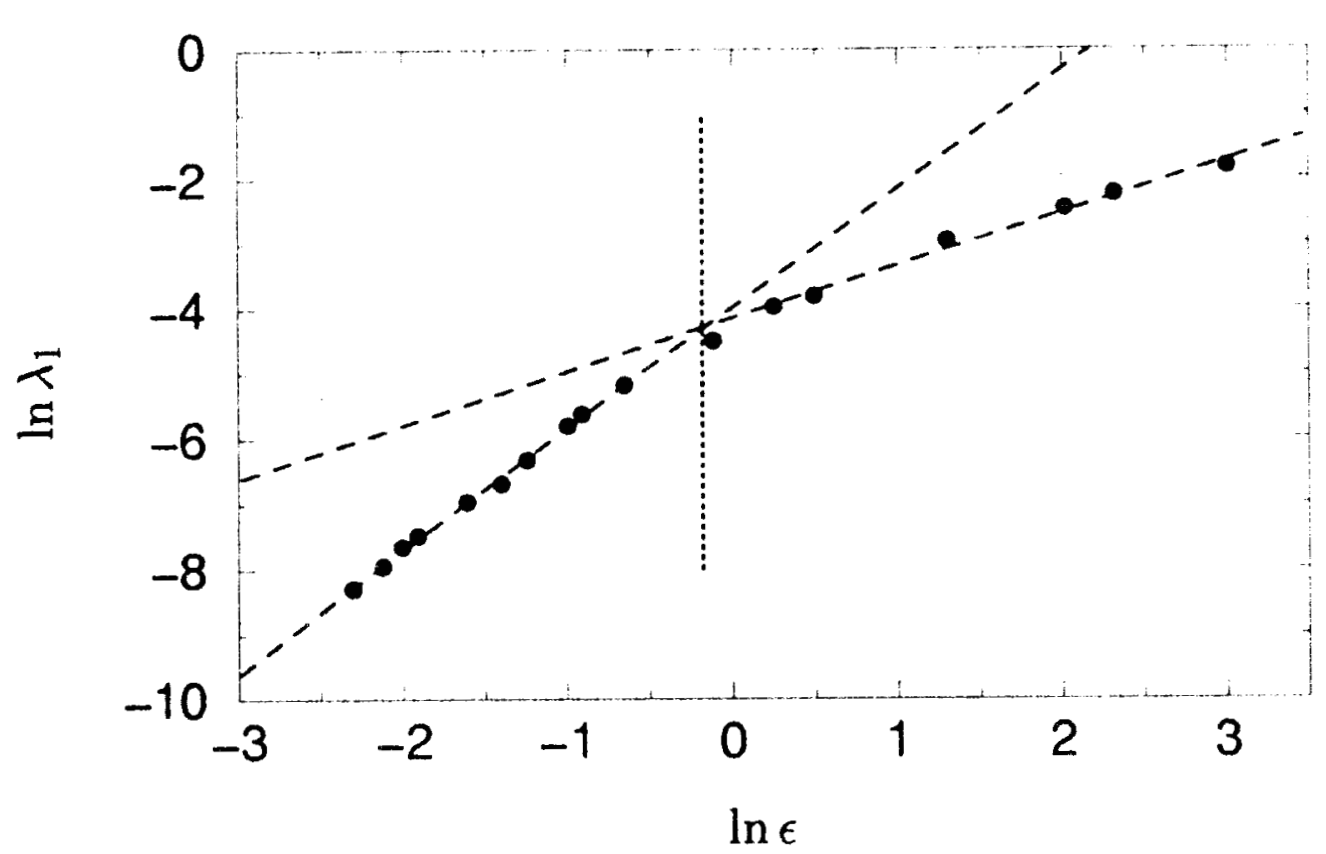

Figura 7.1: Máximo exponente de Lyapunov $\lambda_{1}$ vs densidad de energía $\epsilon$ para el modelo FPU- $\beta$ de masas iguales y $N=128$. Los escalamientos $\epsilon^{2}$ y $\epsilon^{2 / 3}$ están representados por las lineas rectas, mientras que la recta vertical punteada indica la posición de $\epsilon_{c}$. Datos obtenidos de la Ref. [67].

En relación a esta última propuesta podríamos explorar con más detalle la posible existencia de ruido multiplicativo para el caso $\epsilon \geq 1$ y si influye en el ritmo de termalización de nuestro sistema en ese régimen. Además, si se desea simplificar el acoplamiento entre el oscilador pesado y el baño, es posible hacerlo de manera inmediata modificando el Hamiltoniano (5.4) de tal manera que las constantes de acoplamiento entre el oscilador pesado y el baño, tanto la armónica como la anarmónica, sean diferentes a las que existen entre los osciladores del baño. Para el caso de osciladores armónicos acoplados este estudio ya ha sido llevado a cabo de manera analítica [127].

Otra linea de investigación podría iniciarse estudiando el efecto de la inclusión de más de un oscilador pesado en la cadena. Recordemos que, según los resultados del capítulo 5, la equilibración de nuestro sistema depende más del número de grados de libertad que a la presencia del potencial anarmónico en el Hamilto- 
niano. Es entonces razonable suponer que, si se incluyen más osciladores pesados en el sistema, la equilibración se dará en una escala de tiempo mucho menor que la existente para un solo oscilador pesado, que ha sido el caso estudiado en este trabajo. Considerando esta hipótesis sería conveniente comparar los tiempos de termalización de este nuevo sistema en los casos armónico y caótico para ver si son semejantes o diferentes a los correspondientes casos de nuestro sistema de un solo oscilador pesado. Una vez que el sistema esté termalizado podría estudiarse el movimiento Browniano de cada oscilador pesado de tal manera que los resultados pudiesen compararse con aquellos ya conocidos para el caso armónico [128]. Además, ese sería el primer paso para modelar a un sistema cristalino tridimensional dopado con impurezas, con lo cual podríamos comprobar si el tiempo de relajación $r$ asociado a la función de autocorrelación del momento de las impurezas presenta un comportamiento similar al obtenido para nuestro sistema unidirnensional (ver figura 6.11). Cabe señalar que ya se conocen resultados sobre la dinámica cańtica de redes cristalinas compuestas de gases nobles [129].

Retomando el problema del flujo de calor y relajación térmica en modelos unidimensionales hay que mencionar que ya ha sido estudiada la influencia de un defecto pesado en la conducción de calor para el caso de una cadena armónica [130]. Más recientemente ha sido estudiado el problema de la relajación térmica de sistemas FPU, tanto en una y dos dimensiones, como función tanto de los términos potenciales cuadrático y cuártico como de la dimensionalidad [131]. Puede resultar interesante ampliar los resultados de estos trabajos estudiando el efecto de la inclusión de defectos pesados para tratar de investigar si esta modificación altera significativamente la fenomenología ya conocida.

También podríamos investigar, a nivel dinámico, cuál es el efecto de una disparidad de masas en sistemas de baja dimensionalidad. Esta posibilidad resulta muy atractiva porque las herramientas que existen para caracterizar el caos en este tipo de sistemas están mucho más desarrolladas en comparación al caso $N \gg 1$; además, está estrechamente relacionada a nuestro trabajo porque, como ya lo mencionamos en el capítulo 4, el Hamiltoniano del modelo Hénon-Heiles [87] puede obtenerse a partir del correspondiente al modelo FPU $-\alpha$ tomando $N=3$, condiciones periódicas y haciendo un reescalamiento de las variables [88]. Como antecedente podemos mencionar que ya se ha estudiado antes el efecto de una disparidad de masas en el modelo de Toda (uno de los pocos modelos con ecuaciones de movimiento no-lineales pero integrables que se conocen) para $N=2$ $y$, en este caso particular, se há encontrado que el sistema deja de ser integrable 
cuando las masas de las partículas no son iguales [132]. Por lo tanto sería inmediato investigar que modificaciones se presentan, con una disparidad de masas, en la fenomenología del modelo Hénon-Heiles, ya ampliamente caracterizada a través del estudio de las secciones de Poincaré de este sistema.

Finalmente, podemos plantear dos posibles lineas de investigación que nos permitirían relacionar nuestro trabajo con desarrollos recientes en el campo de los sistemas dinámicos. En primer lugar mencionernos que ha sido mostrado que el tiempo medio de existencia de ciertos modos localizados está relacionado al tiempo que le toma al sistema alcanzar la equipartición [133]. Sería interesante tratar de averiguar si estos modos localizados tienen alguna influencia en la relajación hacia el equilibrio de nuestro modelo. Por último, mencionemos que una área de investigación de mucho interés en la actualidad es el estudio de la difusión anómala [134], que está caracterizada por una dependencia del desplazamiento cuadrático medio con respecto al tiempo de la forma $\sim t^{\alpha}$, donde $\alpha \neq 1$. Es bien sabido que este comportamiento difusivo está estrechamente relacionado a la coexistencia de regiones ordenadas y caóticas en el espacio fase de los modelos en los cuales se presenta este fenómeno. Una pregunta interesante surge inmediatamente: ¿por qué no hay evidencia de difusión anómala en nuestro modelo? Como ya hemos visto, para valores $\epsilon \approx 1$ ocurre la transición entre el comportamiento regular y caótico, por lo que existe coexistencia entre caos y orden. Sin embargo, de la figura (6.5) no es posible inferir la existencia de difusión anómala en nuestro modelo; para $\epsilon=0.6$ y 1 el comportamiento del desplazamiento cuadrático medio es virtualmente idéntico al de los casos armónico $(\epsilon=0.01)$ y totalmente caótico $(\epsilon=10)$. Esta ausencia de evidencia de difusión anómala podría deberse a que no hemos podido detectarla o que, por alguna razón desconocida, efectivamente no existe en nuestro modelo, aunque estén dadas las condiciones para su existencia. La confirmación o refutación de estas hipótesis puede ser tema de futuras, y posiblemente muy fructiferas, investigaciones. 


\section{Apéndice A}

\section{Obtención del Conjunto Completo $\left\{U_{i}(k)\right\}$}

Para obtener la forma (3.23) de la función de autocorrelación del momento primero reescribamos al Hamiltoniano del sistema (3.1) en la forma matricial

$$
H=\frac{1}{2}\left[\sum_{i=-N / 2}^{N / 2} \frac{P_{i}^{2}}{M_{i}}+\sum_{i, j=-N / 2}^{N / 2} X_{i} \Phi_{i j} X_{j}\right],
$$

donde la estructura de la matriz $\Phi_{i j}$ está dada en térninos de la constante armónica de acoplamiento $\kappa \mathrm{y}$ de las condiciones de frontera utilizadas (periódicas en nuestro caso). A partir de las ecuaciones de Hamilton se obtiene la ecuación de movimiento para los desplazamientos $\left\{X_{i}\right\}$ de la forma

$$
M_{i} \ddot{X}_{i}(t)=-\sum_{j} \Phi_{i j} X_{j}(t) .
$$

Haciendo explícita la dependencia temporal periódica de los desplazamientos $\left\{X_{i}(t)\right\}$ por medio de

$$
X_{i}(t)=U_{i} \exp (-i \omega t),
$$

donde $\left\{U_{i}\right\}$ se conocen como amplitudes reducidas, las ecuaciones de movimiento del sistema se pueden entonces escribir como

$$
\sum_{j} \Phi_{i j} U_{j}=\omega^{2} M_{i} U_{i},
$$

o tambien de la forma

$$
\cdot \sum_{j}\left(\Phi_{i j}-\omega^{2} M_{j} \delta_{i j}\right) U_{j}=0
$$


La condición para que (A.5) tenga soluciones no-triviales está dada por

$$
\operatorname{det}\left(\Phi_{i j}-\omega^{2} M_{j} \delta_{i j}\right)=0
$$

Este determinante representa la llamada ecuación característica cuyas raices $\left\{\omega_{k}^{2}\right\}$ son conocidas como frecuencias propias del sistema. Ahora bien, para cada solución $\omega_{k}^{2}$ tenemos un vector $\left\{U_{i}(k)\right\}$ que corresponde a un modo de vibración con una única frecuencia $\omega_{k}$. Si escribimos la ecuación (A.4) para las raices $k$-ésima y $k^{\prime}$-ésima de la ecuación secular tenemos que

$$
\begin{aligned}
\sum_{j} \Phi_{i j} U_{j}(k) & =\omega_{k}^{2} \sum_{j} M_{j} U_{j}(k) \delta_{i j} \\
\sum_{i} \Phi_{i j} U_{i}\left(k^{\prime}\right) & =\omega_{k^{\prime}}^{2} \sum_{i} M_{i} U_{i}\left(k^{\prime}\right) \delta_{i j}
\end{aligned}
$$

Si a continuación multiplicamos a la primera de estas ecuaciones por $U_{i}\left(k^{\prime}\right)$ y sumamos sobre $i$, a la segunda por $U_{j}(k)$ y sumamos sobre $j$ y restamos ambas, resulta que

$$
\left(\omega_{k}^{2}-\omega_{k^{\prime}}^{2}\right) \sum_{i} M_{i} U_{i}(k) U_{i}\left(k^{\prime}\right)=0
$$

De este último resultado es inmediato obtener la relación general de ortonormalización para los vectores $\left\{U_{i}(k)\right\}$, que se puede escribir de la forma

$$
\sum_{i} M_{i} U_{i}(k) U_{i}^{*}\left(k^{\prime}\right)=\delta_{k k^{\prime}}
$$

Hasta este punto nuestro desarrollo ha sido independiente de los valores especificos de $\left\{M_{i}\right\}$. Para el caso particular que nos interesa $\left(M_{i}=m<M\right.$ si $i \neq 0$ y $M_{0}=M$ ) podemos reescribir a las ecuaciones de movimiento (A.5) de la forma

$$
\sum_{j}\left(L_{i j}-\delta L_{i j}\right) U_{j}=0
$$

donde

$$
L_{i j}=m \omega^{2} \delta_{i j}-\Phi_{i j}^{(0)}
$$

y

$$
\delta L_{i j}=-\omega^{2} m Q \delta_{j 0} \delta_{i j}+\Delta \Phi_{i j} .
$$

$\Phi_{i j}^{(0)}$ corresponde a la matriz de acoplamiento de un cristal perfecto (todas las masas iguales), mientras que $\Delta \Phi_{i j}$ es la parte de la matriz $\Phi_{i j}$ directamente afectada por la presencia del defecto. Ahora, si este último no existiera tendríamos 
que $\delta L_{i j}=0$ y las ecuaciones de movimiento del sistema serían $\sum_{j} L_{i j} U_{j}=0$. Entonces definimos la función de Green de un cristal perfecto como

$$
\sum_{j} L_{i j} G_{j n}=\delta_{i n}
$$

Si multiplicamos (A.11) por la función de Green arriba definida y sumamos sobre $i$ obtenemos

$$
U_{i}=\sum_{n, j} G_{i n} \delta L_{n j} U_{j}
$$

relación a partir de la cual obtendremos la expresión explícita para las amplitudes reducidas de la red imperfecta, como veremos más adelante.

La condición (A.6) para que (A.5) tenga soluciones no-triviales se escribe, en términos de $L_{i j}$ y $\delta L_{i j}$, como

$$
\operatorname{det}\left(L_{i j}-\delta L_{i j}\right)=0
$$

cuyas soluciones son todas las frecuencias del cristal imperfecto. Sin embargo, se sabe que, a partir de la condición de solubilidad de (A.15):

$$
\Delta\left(\omega^{2}\right)=\operatorname{det}\left(\delta_{i j}-\sum_{n} G_{i n} \delta L_{n j}\right)=0,
$$

se obtienen únicamente las frecuencias que estan afectadas por la presencia del defecto (Ref. [54], pg. 365). Ahora bien, el hecho de que no todas las frecuencias se vean afectadas por la presencia del defecto es una consecuencia de que los modos pares $\left(U_{i}=U_{-i}\right)$ tienen un nodo precisamente en el sitio $i=0$ de la red, por lo que no se ven alterados por la impureza localizada en ese sitio. La solución de (A.17) corresponde a los modos normales impares del cristal imperfecto.

Por medio de técnicas de teoría de grupos que utilizan las condiciones generales de invariancia ante una traslación o rotación del cristal como un todo [j4] se puede rescribir el determinante (A.17) de la forma

$$
1+Q m \omega^{2} g(0)=0,
$$

donde $g(0)$ es el valor correspondiente a $i=0$ de la función de Green para un cristal perfecto y que se calcula a partir de

$$
g(i)=\frac{1}{N} \sum_{n=-(N / 2)+1}^{N / 2} \frac{\exp (\mathrm{i} 2 \pi n i / N)}{m \omega^{2}-2 \kappa(1-\cos (2 \pi n / N))} .
$$


La forma explícita de $g(i)$ para este problema es (Ref. [54], p. 433)

$$
g(i)=\frac{\cot (N \phi / 2) \cos i \phi+\sin |i| \phi}{2 \kappa \sin \phi}, \quad 0<\phi<\pi
$$

Utilizando esta última expresión para la función de Green, las soluciones a la ecuación (A.18) son

$$
\phi_{k}=(2 \pi n / N)-(2 / N) \tan ^{-1}\left(Q \tan \left(\phi_{k} / 2\right)\right),
$$

donde $n=1,2, \ldots, N / 2$ cuando se tienen condiciones de frontera periódicas, como es nuestro caso.

La dependencia espacial de las amplitudes reducidas que son modificadas por la presencia del defecto está dada, a partir de (A.15), por

$$
U_{i}(n)=-Q m \omega_{n}^{2} g(i ; n) U_{0}(n),
$$

donde el índice $n$ indica la $n$-ésima solución de (A.21) y la dependencia de $g$ en $n$ entra a través de $\phi k$. De la condición de ortonormalidad $(A, 10)$ tenemos que

$$
m \sum_{i} U_{i}^{2}(k)+Q m U_{0}^{2}(k)=1 .
$$

Si eliminamos $U_{i}(k)$ de esta ecuación con la ayuda de (A.22) encontramos que $U_{0}(k)$ está determinada por

$$
U_{0}^{2}(k)=\left[Q^{2} m^{3} \omega_{k}^{4} \sum_{i} g^{2}(i ; k)+Q m\right]^{-1} .
$$

Utilizando (A.19) y la expresión explícita de $g(n)$ podemos evaluar la suma en esta expresión, lo que resulta en

$$
\begin{aligned}
\sum_{i} g^{2}(i) & =\frac{1}{N} \sum_{i}\left[m \omega^{2}-2 \kappa+2 \kappa \cos (2 \pi n / N)\right]^{-2} \\
& =-\frac{1}{N m} \frac{d}{d \omega^{2}} \sum_{n}\left[m \omega^{2}-2 \kappa+2 \kappa \cos (2 \pi n / N)\right]^{-1} \\
& =\frac{N}{8 \kappa^{2} \sin ^{2} \phi} \csc ^{2} \frac{N \phi}{2}, \quad 0<\phi<\pi
\end{aligned}
$$

donde hemos usado $\omega=\omega_{0} \sin (\phi / 2)$. Podemos simplificar ia ecuación (A.25) si recordamos que no queremos la suma para un valor arbitrario de $\phi$, sino para una de las soluciones a (A.21). Por lo tanto teremos que

$$
\sum_{i} g^{2}(i ; k)=\frac{N}{32 \kappa^{2}} \frac{1+Q^{2} \tan ^{2}\left(\phi_{k} / 2\right)}{Q^{2} \sin ^{4}\left(\phi_{k} / 2\right)}, \quad 0<\phi_{k}<\pi
$$


Cuando sustituimos este resultado en (A.24) y combinamos la expresión resultante con (A.20) y (A.22) obtexemos

$$
U_{i}(k)=\left(\frac{2}{m N}\right)^{1 / 2} \frac{\left[\cos \left(i \phi_{k}\right)-Q \tan \left(\phi_{k} / 2\right) \sin \left(|i| \phi_{k}\right)\right]}{\left[1+Q^{2} \tan ^{2}\left(\phi_{k} / 2\right)\right]^{1 / 2}}, \quad 0<\phi_{k}<\pi \quad \text { (A.27) }
$$

siendo esta la expresión necesaria para evaluar a (3.21), como se menciona en el capítulo 3. 


\section{Apéndice B}

\section{Correspondencia entre Modelos de Corto y Largo Alcance}

Recordemos que la expresión (3.63) de la función de autocorrelación del momento

$$
\rho_{0}(t)=\exp \left(\Gamma_{p} t\right)+\mu_{p}\left(\frac{2}{\pi \omega_{0}^{P} t}\right)^{\frac{1}{2}} \cos \left(\omega_{0}^{p} t-\frac{\pi}{4}\right),
$$

es válida para interacciones de muy largo alcance, por lo que $z \rightarrow 1$. Entonces los parámetros $\omega_{0}^{P}$ y $\Gamma_{P}$, Ecs. (3.58), se pueden escribir, en ese limite, corno

$$
\omega_{0}^{P}=\left(\frac{\kappa}{m \mu_{p}}\right)^{1 / 2} \quad \text { y } \quad \Gamma_{p}=\left(\frac{\kappa \mu_{p}}{m}\right)^{1 / 2}
$$

donde $z=1-2 \mu_{p}$, como ya se mencionó en el capítulo 3. Puesto que $z \rightarrow 1$, entonces $\mu_{p} \rightarrow 0$. A partir de las expresiones (B.2) podemos definir $\mu_{p}$ como

$$
\mu_{P} \equiv \frac{\Gamma_{p}}{\omega_{0}^{P}} \ll 1
$$

Tenemos entonces que el parámetro $\mu_{P}$ cuantifica la magnitud de la contribución no exponencial en (3.63).

Por otro lado, si en la expresión asintótica para $D_{0}(t)$, Ec. (3.30), tomamos la condición $M \gg m$ y definimos $\mu \equiv m / M$ podemos escribir

$$
D_{0}(t)=\exp (\Gamma t)+\mu\left(\frac{2}{\pi \omega_{0}^{3} t^{3}}\right)^{\frac{1}{2}} \sin \left(\omega_{0} t-\frac{\pi}{4}\right)
$$

donde

$$
\Gamma=\left(\frac{m !}{M^{r}}\right) \omega_{0}=\mu \omega_{0} \quad \text { y } \quad \omega_{0}=2\left(\frac{\kappa}{m}\right)^{1 / 2}
$$




\section{A péndice B. Correspondencia entre Modelos de Corto y Largo Alcance}

Para que la contribución exponencial domine el comportamiento asintótico de $D_{0}(t)$ tenemos que hacer $\mu \ll 1$. Por lo tanto, hemos podido definir los parámetros $\mu_{p}$ y $\mu$ que, cuando son pequeños, permiten despreciar asintóticamente las partes oscilatorias de las respectivas funciones de autocorrelación del momento. Es así que ambos modelos tienen cualitativamente el mismo comportamiento asintótico. Falta comprobar que los coeficientes $\Gamma_{p}$ y $\Gamma$ sean iguales en la aproximación mencionada en el capítulo 3.

Para mostrarlo tenemos primero que, para el modelo de Phillipson,

$$
\Gamma_{p}=\mu_{p} \omega_{0}^{P}
$$

mientras que, para el caso de una partícula pesada de masa $M$ inmersa en una cadena de partículas de masa $m$,

$$
\Gamma=\mu \omega_{0}
$$

Ahora bien, si hacemos que una partícula "pesada" de masa $m$ esté inmersa en una cadena de osciladores "livianos" de masa $m \mu_{p} / 4$ tenemos que hacer la identificación $M \rightarrow m$ y $m \rightarrow m \mu_{p} / 4$ en la expresión (B.7) con lo que resulta

$$
\begin{aligned}
\Gamma & =\mu \omega_{0} \\
& =\frac{m}{M}\left(\frac{4 \kappa}{m}\right)^{1 / 2} \\
& \rightarrow \frac{\frac{m \mu_{P}}{1}}{m}\left(\frac{4 \kappa}{\frac{m \mu_{P}}{4}}\right)^{1 / 2} \\
& =\left(\frac{\mu_{p} \kappa}{m}\right)^{1 / 2} \\
& =\Gamma_{P},
\end{aligned}
$$

demostrando así la equivalencia de ambos modelos. Una posible aplicación de este resultado, propuesta por Phillipson, sería justificar la teoría del movimiento Browniano para una partícula dada de un sistema homogéneo, como la originalmente propuesta por Kirkwood [19] y que ya fue mencionada en el capítulo 2. Como se recordará, en esa teoría el coeficiente de fricción está expresado en términos de la autocorrelación de la fuerza total ejercida sobre una cierta partícula del fluido, Ec. (2.48). Cuando la partícula de interés tiene una masa mayor a la de las demás ya vimos que el uso de ésta expresión, en el límite del acoplamiento 
débil, está plenamente justificada. Pero éste no es el caso para un sistema de partículas idénticas. Ahora bien, si pudiera demostrarse que una partícula en un fluido molecular con interacciones de largo alcance tuviera el mismo comportamiento que el del oscilador central en un sistema con interacciones de largo alcance del tipo trabajado por Phillipson, entonces podría justificarse el uso de la expresión (2.48). Sin embargo, los resultados de dinámica molecular de Vogelsang y Hoheisel [36], utilizando un potencial tipo Lennard-Jones, parecen desmentir esta suposición, ya que esitos autores no pudieron calcular el coeficiente de fricción utilizando (2.48). 
142A péndice B. Correspondencia entre Modelos de Corto y Largo Alcance 


\section{Apéndice $\mathrm{C}$}

\section{El Modelo de Mazur y Braun (1964)}

En este apéndice se desarrollará con todo detalle la metodología utilizada por Mazur y Braun [52] para aplicar el límite del acoplamiento débil al modelo de osciladores acoplados independientemente del alcance de la interacción entre ellos, para así recuperar la fenomenología del movimiento Browniano.

\section{C.1 Dinámica del oscilador pesado en un "baño térmico"}

Consideremos un sisteme, unidimensional consistente de $N+1$ osciladores interaccionando por medio de fuerzas armónicas. El Hamiltoniano del sistema es

$$
H=\frac{1}{2}\left[\sum_{i=-N / 2}^{N / 2} \frac{P_{i}^{2}}{M_{i}}+\sum_{i, j=-N / 2}^{N / 2} X_{i} \Phi_{i j} X_{j}\right],
$$

donde $M_{i}, P_{i}$ y $X_{i}$ denotan la masa, el momento y el desplazamiento del $i$-ésimo oscilador, respectivaments. A continuación establecemos que $M_{0}=M$ (oscilador masivo) y $M_{i}=1$ si $i \neq 0$. La matriz $\Phi$ determina la interacción armónica entre los osciladores y es tal que satisface la propiedad

$$
\Phi_{i j}=\Phi_{|i-j|}
$$

la cual establece que la interacción entre cualquier par de osciladores depende sólo de la distancia entre ellos, como es físicamente razonable suponer. 
Si introducimos en la descripción una fuerza externa derivable de un potencial

$$
F_{\mathrm{e}}\left(X_{0}\right)=-\frac{\partial U_{\mathrm{e}}\left(X_{0}\right)}{\partial X_{0}}
$$

actuando sobre el oscilador masivo, las soluciones de las ecuaciones de movimiento pueden escribirse formalmente como

$$
\begin{aligned}
X_{i}(t)= & M_{i}^{-\frac{1}{2}} \sum_{j}\left[\cos \left(\mathbf{D}^{\frac{1}{2}} t\right)\right]_{i j} M_{j}^{\frac{1}{2}} X_{j}(0) \\
& +M_{i}^{-\frac{1}{2}} \sum_{j}\left[\mathbf{D}^{-\frac{1}{2}} \sin \left(\mathbf{D}^{\frac{1}{2}} t\right)\right]_{i j} M_{j}^{-\frac{1}{2}} P_{j}(0) \\
& +M_{i}^{-\frac{1}{2}} \int_{0}^{i} d t^{\prime}\left[\mathbf{D}^{-\frac{1}{2}} \sin \mathrm{D}^{\frac{1}{2}}\left(t-t^{\prime}\right)\right]_{i 0} M^{-\frac{1}{2}} F_{\mathrm{e}}\left(X_{0}\left(t^{\prime}\right)\right)
\end{aligned}
$$

y

$$
\begin{aligned}
P_{i}(t)= & M_{i}^{\frac{1}{2}} \sum_{j}\left[\cos \left(\mathrm{D}^{\frac{1}{2}} t\right)\right]_{i j} M_{j}^{-\frac{1}{2}} P_{j}(0) \\
& -M_{i}^{\frac{1}{2}} \sum_{j}\left[\mathrm{D}^{\frac{1}{2}} \sin \left(\mathrm{D}^{\frac{1}{2}} t\right)\right]_{i j} M_{j}^{\frac{1}{2}} X_{j}(0) \\
& +M_{i}^{\frac{1}{2}} \int_{0}^{t} d t^{\prime}\left[\cos \mathrm{D}^{\frac{1}{2}}\left(t-t^{\prime}\right)\right]_{i 0} M^{-\frac{1}{2}} F_{\mathrm{e}}\left(X_{0}\left(t^{\prime}\right)\right)
\end{aligned}
$$

donde $\mathbf{D}$ es la matriz dinámica cuyos elementos están dados por

$$
\mathrm{D}_{i j}=M_{i}^{-\frac{1}{2}} \Phi_{i j} M_{j}^{-\frac{1}{2}}
$$

y las funciones trigonométricas $\cos \left(D^{\frac{1}{2}} t\right)$, etc. están definidas por sus desarrollos en serie. La matriz $\Phi$ debe además satisfacer la relación

$$
\sum_{j} \Phi_{j i}=M_{i}^{\frac{1}{2}} \sum_{j} M_{j}^{\frac{1}{2}} D_{j i}=0,
$$

misma que garantiza la conservación del momento total del sistema.

Para obtener la ecuación de movimiento del oscilador pesado diferenciamos $P_{0}(t)$ con respecto al tiempo en la ecuación (C.5), con lo cual

$$
\begin{aligned}
\dot{P}_{0}(t)= & -\left(\mathbf{D}^{\frac{1}{2}} \sin \mathbf{D}^{\frac{1}{2}} t\right)_{00} P_{0}(0)-M^{\frac{1}{2}} \sum_{j \neq 0}\left(\mathbf{D}^{\frac{1}{2}} \sin \mathbf{D}^{\frac{1}{2}} t\right)_{0 j} P_{j}(0) \\
& -M^{\frac{1}{2}} \sum_{j}\left(\mathbf{D} \cos \mathbf{D}^{\frac{1}{2}} t\right)_{0 j} M_{j}^{\frac{1}{2}} X_{j}(0)+F_{\mathrm{e}}\left(X_{0}(t)\right) \\
& -\int_{0}^{t} d t^{\prime}\left(\mathbf{D}^{\frac{1}{2}} \sin \mathbf{D}^{\frac{1}{2}}\left(t-t^{\prime}\right)\right)_{00} F_{\mathrm{e}}\left(X_{0}\left(t^{\prime}\right)\right)
\end{aligned}
$$


Como siguiente paso despejamos, de la misma ecuación (C.5), a $P_{0}(0)$ tomando $j=0$ y cambiando los írdices $j \rightarrow i$. El resultado es

$$
\begin{aligned}
P_{0}(0)= & \left(\cos \mathbf{D}^{\frac{1}{2}} t\right)_{00}^{-1} P_{0}(t)-M^{\frac{1}{2}} \sum_{i \neq 0} \frac{\left(\cos \mathbf{D}^{\frac{1}{2}} t\right)_{0 i}}{\left(\cos \mathbf{D}^{\frac{1}{2}} t\right)_{00}} P_{i}(0) \\
& +M \frac{\left(\mathbf{D}^{\frac{1}{2}} \sin \mathbf{D}^{\frac{1}{2}} t\right)_{00}}{\left(\cos \mathbf{D}^{\frac{1}{2}} t\right)_{00}} X_{0}(0)+M^{\frac{1}{2}} \sum_{i \neq 0} \frac{\left(\mathbf{D}^{\frac{1}{2}} \sin \mathbf{D}^{\frac{1}{2}} t\right)_{0 i}}{\left(\cos \mathbf{D}^{\frac{1}{2}} t\right)_{00}} X_{i}(0) \\
& -\left(\cos \mathbf{D}^{\frac{1}{2}}\right)_{00}^{-1} \int_{0}^{t} d t^{\prime}\left[\cos \mathbf{D}^{\frac{1}{2}}\left(t-t^{\prime}\right)\right]_{00} F_{\mathbf{e}}\left(X_{0}\left(t^{\prime}\right)\right) .
\end{aligned}
$$

Sustituyendo este resulttdo en (C.8) obtenemos

$$
\begin{aligned}
& \dot{P}_{0}(t)-F_{\mathrm{e}}\left(X_{0}(t)\right)=-\gamma(t) P_{0}(t)+F(t) \\
& \quad+\int_{0}^{t} d t^{\prime}\left(\gamma(t)-\gamma\left(t-t^{\prime}\right)\right)\left(\cos \mathrm{D}^{\frac{1}{2}}\left(t-t^{\prime}\right)\right)_{00} F_{\mathrm{e}}\left(X_{0}\left(t^{\prime}\right)\right),
\end{aligned}
$$

donde $\gamma(t)$ es un "coeficiente de fricción" dependiente del tiempo dado por

$$
\gamma(t)=\frac{\left(\mathbf{D}^{\frac{1}{2}} \sin \mathbf{D}^{\frac{1}{2}} t\right)_{00}}{\left(\cos \mathbf{D}^{\frac{1}{2}} t\right)_{00}}=-\frac{d}{d t} \ln \left(\cos \mathbf{D}^{\frac{1}{2}} t\right)_{00}
$$

y $F(t)$ es una fuerza que tiene la forma

$$
\begin{aligned}
F(t)= & M^{\frac{1}{2}} \sum_{i \neq 0}\left[\gamma(t)\left(\cos \mathbf{D}^{\frac{1}{2}} t\right)_{0 i}-\left(\mathbf{D}^{\frac{1}{2}} \sin \mathbf{D}^{\frac{1}{2}} t\right)_{0 i}\right] P_{i}(0) \\
& -M^{\frac{1}{2}} \sum_{i \neq 0}\left[\gamma(t)\left(\mathbf{D}^{\frac{1}{2}} \sin \mathbf{D}^{\frac{1}{2}} t\right)_{0 i}+\left(\mathbf{D} \cos \mathbf{D}^{\frac{1}{2}} t\right)_{0 i}\right] X_{i}(0) \\
& -M \gamma\left(t_{j}^{\prime}\left(\mathbf{D}^{\frac{1}{2}} \sin \mathbf{D}^{\frac{1}{2}} t\right)_{00} X_{0}(0)-M\left(\mathbf{D} \cos \mathbf{D}^{\frac{1}{2}} t\right)_{00} X_{0}(0) .\right.
\end{aligned}
$$

Si en esta última expresión usamos (C.7) podemos reescribir a la fuerza $F(t)$ como

$$
\begin{aligned}
F(t)= & M^{\frac{1}{2}} \sum_{i \neq 0}\left[\gamma(t)\left(\cos \mathbf{D}^{\frac{1}{2}} t\right)_{0 i}-\left(\mathbf{D}^{\frac{1}{2}} \sin \mathbf{D}^{\frac{1}{2}} t\right)_{0 i}\right] P_{i}(0) \\
& -M^{\frac{1}{2}} \sum_{i \neq 0}\left[\gamma(t)\left(\mathbf{D}^{\frac{1}{2}} \sin \mathbf{D}^{\frac{1}{2}} t\right)_{0 i}+\left(\mathbf{D} \cos \mathbf{D}^{\frac{1}{2}} t\right)_{0 i}\right]\left(X_{i}(0)-X_{0}(0)\right) .
\end{aligned}
$$

Obsérvese que la fuerza $F^{\prime}(t)$ no depende de la fuerza externa $F_{\mathrm{e}}\left(X_{0}\right)$, sino sólo del estado inicial de los $N$ osciladores de masa unitaria, los cuales pueden considerarse como el "medio" que rodea al oscilador pesado. Este estado inicial está determinado por los momentos iriciales y los desplazamientos relativos de los osciladores livianos respecto al oscilador pesado. 
A continuación supongamos que las condiciones iniciales del oscilador pesado $\left\{P_{0}(0), X_{0}(0)\right\}$ son tales que están en equilibrio termodinámico con respecto al "medio" en el instante $t=0$. Esto equivale a considerar al conjunto de osciladores livianos como un "baño térmico" en el cual está sumergido el oscilador pesado. Entonces el así definido "baño térmico" puede ser descrito por una función de distribución canónica no-estacionaria

$$
f_{\mathrm{ne}}=\exp \left(-\mathcal{H} / k_{B} T\right)
$$

donde $T$ es la temperatura del "baño térmico" y

$$
\mathcal{H}=\frac{1}{2}\left[\sum_{i, 00} P_{i}^{2}+\sum_{i, j \neq 0}\left(X_{i}-X_{0}\right) \Phi_{i, j}\left(X_{j}-X_{0}\right)\right] .
$$

Debido a la elección de variables en este último Hamiltoniano vemos, a partir de (C.13), que $F(t)$ es una suma de variables aleatorias Gaussianas, siendo entonces ella misma un proceso aleatorio Gaussiano.

Como $F(t)$ no depende de $F_{\mathrm{e}}\left(X_{0}(t)\right)$, en la ecuación de movimiento (C.10) podemos tomar $F_{\mathrm{e}}\left(X_{0}(t)\right)=0$, despejar $F(t)$ y calcular así la función de autocorrelación para esta fuerza. Utilizando el ensamble no-estacionario definido por (C.14) el resultado es

$$
\begin{aligned}
\left\langle\left\langle F\left(t_{0}\right) F\left(t_{0}-t\right)\right\rangle\right\rangle= & -\frac{\partial^{2}}{\partial t^{2}} \rho_{0}^{*}(t)+\gamma\left(t_{0}\right) \gamma\left(t_{0}+t\right) \rho_{0}^{*}(t) \\
& +\left[\gamma\left(t_{0}\right)-\gamma\left(t_{0}+t\right)\right] \frac{\partial}{\partial t} \rho_{0}^{*}(t),
\end{aligned}
$$

donde $\langle(\cdots)\rangle$ denota un promedio usando la función de distribución (C.14), mieritras que la función de autocorrealción del momento del oscilador pesado en ausencia de fuerzas externas, $\rho_{0}^{*}(t)$, está definida como

$$
\rho_{0}^{*}(t) \equiv\left\langle P_{0}(0) P_{0}(t)\right\rangle
$$

En esta última expresión $\langle.$.$\rangle denota un promedio tomado con la función de$ distribución estacionaria

$$
f_{\mathrm{e}}=\exp \left(-H / k_{B} T\right)
$$

con $H$ dada por (C.1). El cambio de promedio puede hacerse porque $F(t)$ no depende del valor de $P_{0}(0)$. Ahrora bien, para escribir la forma explícita de la función de autocorrelación del momento $\rho_{0}^{*}(t)$ en términos de la solución exacta 
ya anteriormente obtenida multiplicamos la ecuación de movimiento (C.5) por $P_{0}(0)$ tomando además $F_{\mathrm{e}}\left(X_{0}(t)\right)=0$, con lo que obtenemos

$$
\begin{aligned}
P_{0}(0) P_{0}(t)= & P_{0}^{2}(0)\left(\cos \mathbf{D}^{\frac{1}{2}} t\right)_{00}+M^{\frac{1}{2}} \sum_{i \neq 0}\left(\cos \mathrm{D}^{\frac{1}{2}} t\right)_{0 i} P_{0}(0) P_{i}(0) \\
& -M^{\frac{1}{2}} \sum_{i}\left(\mathbf{D}^{\frac{1}{2}} \sin \mathbf{D}^{\frac{1}{2}} t\right)_{0 i} M_{i}^{\frac{1}{2}} X_{i}(0) P_{0}(0) .
\end{aligned}
$$

Puesto que las condiciones iniciales de los osciladores del "baño" son Gaussianas, al tomar el promedio $(\cdots)$ en ambos lados de la expresión anterior los últimos dos sumandos del lado derecto se eliminan, pues

$$
\left\langle P_{0}(0) P_{i}(0)\right\rangle=\left\langle P_{0}(0) X_{i}(0)\right\rangle=0 . \quad i \neq 0
$$

Entonces

$$
\rho_{0}^{*}(t):=\left\langle P_{0}(0) P_{0}(t)\right\rangle=\left(\cos \mathrm{D}^{\frac{1}{2}} t\right)_{00}\left\langle P_{0}^{2}(0)\right\rangle .
$$

Utilizando (C.1) podemos calcular $\left\langle P_{0}^{2}(0)\right\rangle$ como

$$
\left\langle P_{0}^{2}(0)\right\rangle=\frac{\int_{-\infty}^{+\infty} P^{2} \exp \left(-P^{2} / 2 M k_{B} T\right) d P}{\int_{-\infty}^{+\infty} \exp \left(-P^{2} / 2 M k_{B} T\right) d P}=M k_{B} T
$$

obteniendo finalmente la expresión para la función de autocorrelación del momento

$$
\rho_{0}^{*}(t)=M k_{B} T\left(\cos \mathrm{D}^{\frac{1}{2}} t\right)_{00},
$$

misma que resulta muy semejante a la expresión (3.46) utilizada por Ford et al.. Sin embargo, (C.22) es más complicada debido a que está escrita en términos de la matriz dinámica $\mathbf{D}$ característica de un cristal con un isótopo masivo en la posición $i=0$

\section{C.2 Limite dell Acoplamiento Débil}

Ahora procederemos a aplicar el límite del acoplamiento débil al cálculo de elemento 00 de la matriz $\cos \mathrm{D}^{\frac{1}{2}} t$ para así poder calcular la función de autocorrelación del momento (C.22) y demostrar que la misma tiene un decaimiento estrictamente exponencial, como ya mencionamos en el capítulo 3 . En el límite $N \rightarrow \infty$ podemos 
escribir

$$
\begin{aligned}
\left(\cos \mathbf{D}^{\frac{1}{2}} t\right)_{00} & =\left(\cos M \mathrm{D}^{\frac{1}{2}} \tau\right)_{\infty \omega} \\
& =\int_{-\infty}^{+\infty} d \omega \cos \omega \tau\left[\delta\left(M \mathbf{D}^{\frac{1}{2}}-\omega \mathbf{I}\right)\right]_{\omega 0} \\
& =\int_{-\infty}^{+\infty} d \omega \cos \omega \tau S_{M}(\omega)
\end{aligned}
$$

donde

$$
\tau=t / M
$$

y el espectro $S_{M}(\omega)$ está definido como

$$
S_{M}(\omega)=\frac{1}{2}\left[\delta\left(M \mathbf{D}^{\frac{1}{2}}-\omega \mathbf{I}\right)+\delta\left(M \mathbf{D}^{\frac{1}{2}}+\omega \mathbf{I}\right)\right\}_{(0)} .
$$

El escalamiento en el tiempo (C.24) fue introducido para poder estudiar el movimiento del oscilador pesado en el límite $M \rightarrow \infty$ puesto que, en la escala original de tiempo, un oscilador infinitamente pesado no reaccionaría en absoluto a la presencia del "nedio". Si además tomamos el límite $t \rightarrow \infty$ de tal manera que el tiempo escalado $\tau$ permanezca finito, entonces tenemos una aplicación particular del límite del acoplamiento débil ya mencionado en el capítulo 2.

A partir de la representación

$$
\lim _{\epsilon \rightarrow 0^{+}} \frac{1}{u \mp \mathrm{i} \epsilon}=\mathcal{P}\left(\frac{1}{u}\right) \pm \mathrm{i} \pi \delta(u),
$$

donde $\mathcal{P}$ denota el valor principal (ver Ref. $[135], \S 8$ ) podemos obtener las siguientes representaciones de la función delta

$$
\delta\left(M \mathbf{D}^{\frac{1}{2}}-\omega \mathbf{I}\right)=\frac{1}{\pi} \operatorname{Im} \lim _{\epsilon \rightarrow 0^{+}} \frac{1}{M \mathbf{D}^{\frac{1}{2}}-(\omega+\mathrm{i} \epsilon) \mathbf{I}}
$$

y

$$
\delta\left(M \mathrm{D}^{\frac{1}{2}}+\omega \mathbf{I}\right)=-\frac{1}{\pi} \operatorname{Im} \lim _{\epsilon \rightarrow 0^{+}} \frac{1}{M \mathrm{D}^{\frac{1}{2}}+(\omega+\mathrm{i} \epsilon) \mathrm{I}},
$$

las cuales nos permiten escribir el espectro (C.25) como

$$
S_{M}(\omega)=\operatorname{Im} \lim _{\epsilon \rightarrow 0^{+}} \frac{1}{\pi} \frac{1}{M^{2}}(\omega+\mathrm{i} \epsilon)\left[\mathrm{D}-\left(\frac{\omega+\mathrm{i} \epsilon}{M}\right)^{2} \mathrm{I}\right]_{00}^{-1} .
$$

\footnotetext{
${ }^{\prime}$ Aunque la nomenclatura es parecida, no hay que confundir este espectro $S_{M}(\omega)$ con el espectro de frecuencias $g(\omega)$ ya definido en el capitilo 3 y que posteriormente utilizaremos.
} 
Definiendo la resolvente de la matriz dinámica $\mathrm{D}$ como

$$
\mathbf{R}\left(z^{2}\right)=\left(\mathbf{D}-z^{2} \mathbf{I}\right)^{-1},
$$

vemos entonces que el espectro $S_{M}(\omega)$ toma la siguiente forma

$$
S_{M}(\omega)=\operatorname{Im} \lim _{\epsilon \rightarrow 0^{+}} \frac{1}{\pi} \frac{1}{M^{2}}(\omega+\mathrm{i} \epsilon) \mathrm{R}_{00}\left(\left(\frac{\omega+\mathrm{i} \epsilon}{M}\right)^{2}\right) .
$$

La utilidad de esta expresión para $S_{M}(\omega)$ es que también podemos definir una resolvente para la matriz $\Phi$ como

$$
\mathbf{Q}\left(z^{2}\right)=\left(\mathbf{\Phi}-z^{2} \mathbf{I}\right)^{-1} .
$$

Si además recordamos la relación (C.6) entre las matrices $D$ y $\Phi$, entonces es claro que podemos obtener una relación entre los elementos 00 de las resolventes $\mathbf{R}$ y Q. Para esto reescribimo; (C.6) en forma matricial

$$
\mathbf{D}=\mathbf{M}^{-\frac{1}{2}} \boldsymbol{\Phi} \mathbf{M}^{-\frac{1}{2}}
$$

siendo $\mathbf{M}$ una matriz diagonal con elementos $M_{j \delta_{j i}}$. Si tomamos la forma equivalente

$$
\mathbf{M}^{\frac{1}{2}} \mathbf{D}=\boldsymbol{\Phi} \mathbf{M}^{-\frac{1}{2}},
$$

despejamos de (C.30) y (C.32) D y $\Phi$, respectivamente y sustituimos en (C.34), encontramos que

$$
\mathbf{M}^{\frac{1}{2}}\left(\mathbf{R}^{-1}+z^{2} \mathbf{I}\right)=\left(\mathbf{Q}^{-1}+z^{2} \mathbf{I}\right) \mathbf{M}^{-\frac{1}{2}} .
$$

A continuación multiplicamos (C.35) a la izquierda por $\mathbf{Q}$ y a la derecha por $\mathbf{R}$; despejando $\mathbf{R}$ y tomando el elemento 00 se encuentra la relación buscada entre los elementos 00 de las resolventes de las matrices $\mathbf{D}$ y $\Phi$ de la forma

$$
\mathrm{R}_{100}\left(z^{2}\right)=\frac{M Q_{00}\left(z^{2}\right)}{1+z^{2}(1-M) Q_{00}\left(z^{2}\right)} .
$$

Puesto que deseamos tomar el límite $M \rightarrow \infty$, en esta última expresión hacemos el cambio de variable $z^{2} \rightarrow z^{2} / M^{2}$, multiplicamos ambos lados por $z / M^{2}$ y entonces tomamos el límite $M \rightarrow \infty$ para obtener finalmente

$$
\lim _{M \rightarrow \infty} \frac{1}{M^{2}} z \mathrm{~F}_{00}\left(\frac{z^{2}}{M^{2}}\right)=\frac{\lim _{M \rightarrow \infty} z M^{-1} \mathrm{Q}_{00}\left(\frac{z^{2}}{M^{2}}\right)}{1-z \lim _{M \rightarrow \infty} z M^{-1} \mathrm{Q}_{00}\left(\frac{z^{2}}{M^{2}}\right)} .
$$


Nuestro siguiente paso consistirá en expresar $Q_{00}$ en términos del elemento 00 de la matriz $\Phi$. Este objetivo se consigue desarrollando la resolvente $\mathbf{Q}$ en serie de potencias a partir de la definición (C.32) como

$$
\begin{aligned}
\mathbf{Q}\left(z^{2}\right) & =\left(\boldsymbol{\Phi}-z^{2} \mathbf{I}\right)^{-1} \\
& =-\frac{1}{z^{2}}\left(\mathbf{I}-\frac{1}{z^{2}} \boldsymbol{\Phi}\right)^{-1} \\
& =-\frac{1}{z^{2}}\left(\mathbf{I}+\frac{1}{z^{2}} \boldsymbol{\Phi}+\frac{1}{z^{4}} \boldsymbol{\Phi}^{2}+\ldots\right),
\end{aligned}
$$

del cual es inmediato obtener el clemento de matriz deseado

$$
Q_{00}\left(z^{2}\right)=-\frac{1}{z^{2}}\left((\mathbf{I})_{00}+\frac{1}{z^{2}}(\boldsymbol{\Phi})_{00}+\frac{1}{z^{4}}\left(\boldsymbol{\Phi}^{2}\right)_{00}+\ldots\right) .
$$

Ahora bien, como ya se mencionó en el capítulo 3, en el límite $N \rightarrow \infty$ los elementos de la matriz $\Phi$ se pueden expresar como la transformada de Fourier de una cierta función $f(9)$. Esto es,

$$
\Phi_{i j}=\frac{1}{2 \pi} \int_{-\pi}^{\pi} d \theta f(\theta) \exp (\mathrm{i}(i-j) \theta) .
$$

Puesto que deseamos asegurarnos que $\mathbf{\Phi}$ sea

- simétrica positiva,

- semi-definida y

- con la propiedad (C.7),

tenemos entonces que utilizar una función $f(\theta)$ que sea

- $f(\theta)>0$

- $f(-\theta)=f(\theta)$,

- $f(0)=0$,

- acotada y

- monotónica por partes. 
Suponiendo que podaraos encontrar una $f(\theta)$ que satisfaga las propiedades enumeradas arriba, de la cefinición (C.39) podemos calcular cada uno de los elementos de la serie (C.38). Por ejemplo, el primero es

$$
\mathbf{I}_{00}=1=\frac{1}{2 \pi} \int_{-\pi}^{\pi} d \theta,
$$

el segundo tiene la forma

$$
(\Phi)_{00}=\frac{1}{2 \pi} \int_{-\pi}^{\pi} d \theta f(\theta),
$$

mientras que el tercero se puede calcular como

$$
\begin{aligned}
\left(\Phi^{2}\right)_{00} & =\lim _{N \rightarrow \infty} \sum_{i=-N / 2}^{N / 2} \Phi_{0 i} \Phi_{i 0} \\
& =\frac{1}{(2 \pi)^{2}} \lim _{N \rightarrow \infty} \sum_{i=-N / 2}^{N / 2} \int_{-\pi}^{\pi} \int_{-\pi}^{\pi} d \theta_{1} d \theta_{2} f\left(\theta_{1}\right) f\left(\theta_{2}\right) \exp \left(-\mathrm{i} \theta_{1} i\right) \exp \left(\mathrm{i} \theta_{2} i\right) \\
& =\frac{1}{(2 \pi)^{2}} \int_{-\pi}^{\pi} \int_{-\pi}^{\pi} d \theta_{1} d \theta_{2} f\left(\theta_{1}\right) f\left(\theta_{2}\right) \lim _{N \rightarrow \infty} \sum_{i=-N / 2}^{N / 2} \exp \left(\mathrm{i}\left(\theta_{2}-\theta_{1}\right) i\right) \\
& =\frac{1}{2 \pi} \int_{-\pi}^{\pi} d \theta_{1} f^{2}\left(\theta_{1}\right)
\end{aligned}
$$

si utilizamos la identidad

$$
\lim _{N \rightarrow \infty} \sum_{i=-N / 2}^{N / 2} \exp (\mathrm{i} \theta i)=2 \pi \delta(\theta),
$$

y asi sucesivamente. Teniendo en cuenta estos resultados podemos escribir $Q_{00}$ como

$$
\begin{aligned}
\mathrm{Q}_{00}\left(z^{2}\right) & =-\frac{1}{z^{2}}\left(\frac{1}{2 \pi} \int_{-\pi}^{\pi} d \theta+\frac{1}{z^{2}} \frac{1}{2 \pi} \int_{-\pi}^{\pi} d \theta f(\theta)+\frac{1}{z^{4}} \frac{1}{2 \pi} \int_{-\pi}^{\pi} d \theta f^{2}(\theta)+\ldots\right) \\
& =\frac{1}{2 \pi} \int_{-\pi}^{\pi} d \theta\left[-\frac{1}{z^{2}}\left(1+\frac{f(\theta)}{z^{2}}+\frac{f^{2}(\theta)}{z^{4}}+\ldots\right)\right] \\
& =\frac{1}{2 \pi} \int_{-\pi}^{\pi} d \theta\left[-\frac{1}{z^{2}}\left(1-\frac{f(\theta)}{z^{2}}\right)^{-1}\right]
\end{aligned}
$$

o bien,

$$
Q_{30}\left(z^{2}\right)=\frac{1}{2 \pi} \int_{-\pi}^{\pi} d \theta \frac{1}{f(\theta)-z^{2}} .
$$


Por medio del cambio de variable $\omega^{2}=f(\theta)$ podemos escribir la ecuación anterior de la siguiente forma

$$
Q_{\omega 0}\left(z^{2}\right)=\frac{1}{2} \int_{-\omega_{0}}^{\omega_{0}} d \omega \frac{g(\omega)}{\omega^{2}-z^{2}},
$$

donde $g(\omega)$ es el espectro de frecuencias positivas de los modos normales en un sistema de osciladores con masas idénticas y cuya forma explícita puede calcularse a partir de (3.61) para el caso de una matriz $\Phi$ de interacción específica.

Realizando el cambio de variable $z \rightarrow z / M$ y multiplicando la última expresión obtenida para $Q_{00}$ por $z / M$ obtenemos

$$
z M^{-1} Q_{00}\left(\frac{z^{2}}{M^{2}}\right)=\frac{1}{2} \int_{-\omega_{0}}^{\omega_{0}} d \omega g(\omega) \frac{1}{2}\left\{\frac{1}{\omega-z / M}-\frac{1}{\omega+z / M}\right\} .
$$

De la definición (C.26) que nos permitió escribir (C.27) y (C.28) tenemos

$$
\lim _{M \rightarrow \infty} \frac{1}{\omega \mp z / M}=\mathcal{P}\left(\frac{1}{\omega}\right) \pm i \pi \delta(\omega),
$$

Utilizando esta expresión en (C.45) encontramos que

$$
\lim _{M \rightarrow \infty} z M^{-1} Q_{00}\left(\frac{z^{2}}{M^{2}}\right)=\mathrm{i} \frac{\pi}{2} g(0) .
$$

Si a continuación sustituimos (C. 47$)$ en (C.37) obtenemos

$$
\lim _{M \rightarrow \infty} \frac{1}{M^{2}} z \mathrm{R}_{00}\left(\frac{z^{2}}{M^{2}}\right)=\frac{\mathrm{i} \frac{\pi}{2} g(0)}{1-\mathrm{i} \frac{\pi}{2} g(0) z} .
$$

Haciendo uso de este último resultado tenemos que la expresión (C.31) para el espectro $S_{M}(\omega)$ puede escribirse de la siguiente manera

$$
S_{M}(\omega)=\operatorname{Im} \frac{1}{\pi} \frac{i \frac{\pi}{2} g(0)}{1-i \frac{\pi}{2} g(0) \omega} .
$$

Si ahora escribimos explícitamente la parte imaginaria obtenemos

$$
S_{M}(\omega)=\frac{1}{\pi} \frac{\Gamma_{M}}{\Gamma_{M}^{2}+\omega^{2}}
$$

donde

$$
\Gamma_{M}=\frac{2}{\pi g(0)}
$$


Es claro entonces que $g(0) \neq 0$ es la única (y no muy restrictiva) condición que se establece sobre el espectro de frecuencias de los modos normales para que el coeficiente $\Gamma_{M}$ esté bien definido. Por lo demás la matriz $\Phi$ es totalmente arbitraria, lo cual implica que la especificación detallada del "medio" no es necesaria para obtener el movimiento Browniano en el límite del acoplamiento débil.

Finalmente, sustituyendo el resultado (C.50) en la expresión (C.23) obtenemos

$$
\lim _{\substack{M \rightarrow \infty \\ t \rightarrow M \infty \\ t / M=\tau}}\left(\cos D^{\frac{1}{2}} t\right)_{00}=\exp \left(-\Gamma_{M}|\tau|\right)
$$

el cual nos permite calcular el "coeficiente de fricción" (C.11) como

$$
\lim _{M \rightarrow \infty} M \gamma(\tau)=\Gamma_{M} \quad \tau>0
$$

En este punto de nuestro desarrollo es claro que se recuperan los resultados ya conocidos de la fenomenología del movimiento Browniano, puesto que la función $\gamma(t)$ que aparece en (C.10) se convierte, en el límite de masa infinita, en una constante positiva. De igual manera, tomando ese mismo límite en (C.10), esta ecuación se reduce asintóticamente a la ecuación de Langevin

$$
\dot{P}_{0}(\tau)=-\Gamma_{M} P_{0}(\tau)+\lim _{M \rightarrow \infty}\left[M F(\tau)+M F_{\mathrm{e}}\left(X_{0}(\tau)\right)\right], \quad \tau>0
$$

donde ahora $F(\tau)$ es una fuerza estocástica Gaussiana. Para comprobarlo partimos de (C.16). En el límite de masa infinita el tercer sumando de esa expresión es cero en virtud de (C.52) y (C.53). Si ahora sustituimos en los sumandos que quedan (C.23), tomamos el límite ya mencionado y usamos (C.50), tenemos finalmente que

$$
\lim _{M \rightarrow \infty} M\left\langle F\left(\tau_{0}\right) F\left(\tau_{0}+\tau\right)\right\rangle=2 \Gamma_{M} k_{B} T \delta(\tau) .
$$

Ahora bien, es claro que (C.54) y (C.55) no tienen exactamente la misma forma de la ecuación fenomenológica de Langevin y de la autocorrelación de la fuerza estocástica, respectivamente; la semejanza se vuelve identidad sólo en el límite de masa infinita. Sin embargo, para el caso de interacciones a primeros vecinos, el coeficiente de fricción $\Gamma_{M}$ es idéntico a aquel calculado en el capítulo 3. Para comprobarlo tenemos que partir de la forma explícita de la matriz $\Phi$ para el caso de primeros vecinos y condiciones de frontera periódicas

$$
\Phi_{i j}=2 \delta_{i, j}-\delta_{i, j+1}-\delta_{i, j-1},
$$


utilizar (C.39) para calcular de ahí, por inversión, la función $f(\theta)$ y sustituir la expresión resultante en (3.61) para obtener finalmente la densidad de frecuencias $g(\omega)$, cuya forma explícita está dada por (3.59) como ya lo habíamos mencionado. Si ahora sustituimos $g(0)$ en (C.51) obtenemos de manera trivial que

$$
\Gamma_{M}=\omega_{0}
$$

Para el caso de primeros vecinos tenemos que la expresión asintótica (3.31) de la correspondiente función $\gamma(t)$ está dada por

$$
\gamma(t) \rightarrow \frac{\omega_{0}}{\left(Q^{2}-1\right)^{1 / 2}} \quad \text { para } \quad t \gg \omega_{0}^{-1}
$$

donde $Q=M / m-1$. Si ambos esquemas dan las mismos resultados, entonces $\gamma(t)$ debe de satisfacer

$$
\lim _{M \rightarrow \infty} M \gamma(t)=\omega_{0}
$$

que es un límite análogo a (C.53). 'Tomando $m=1$ la demostración es inmediata:

$$
\begin{aligned}
\lim _{M \rightarrow \infty} M \gamma(t) & =\lim _{M \rightarrow \infty} \frac{M \omega_{0}}{\left[(M-1)^{2}-1\right]^{1 / 2}} \\
& \simeq \lim _{M \rightarrow \infty} \frac{M \omega_{0}}{\left[M^{2}\right]^{1 / 2}} \\
& =\omega_{0},
\end{aligned}
$$

lo que implica que $\Gamma_{M}=\Gamma$. Queda comprobado que, en el límite del acoplamiento débil, el modelo de prineros vecinos se vuelve un caso particular del Hamiltoniano (C.1). 


\section{Apéndice D}

\section{Algoritmo de Integración}

Para poder integrar numéricamente las ecuaciones de movimiento (5.5), que escribimos como

$$
\frac{d^{2}}{d t^{2}} X_{i}(t)=G_{i}\left(\left\{X_{i}(t)\right\}\right)
$$

observamos, en primer lugar, que son invariantes ante una inversión temporal; esto es, si $t \rightarrow-t$, entonces las velocidades del sistema cambian como $V_{i} \rightarrow-V_{i}$ y (D.1) permanece inalterada. Esta caracteristica es fundamental en el desarrollo que a continuación presentamos.

Sea $\Delta t$ un intervalo de tiempo pequeño. Si realizamos dos desarrollos en serie de Taylor, uno hacia adelante y otro hacia atrás en el tiempo, obtenemos, al sustituir (D.1), las siguientes expresiones

$$
\begin{aligned}
X_{i}(t+\Delta t)=X_{i}(t)+ & -\Delta t \frac{d}{d t} X_{i}(t)+\frac{\Delta t^{2}}{2} G_{i}\left(\left\{X_{i}(t)\right\}\right)+\frac{\Delta t^{3}}{6} \frac{d}{d t} G_{i}\left(\left\{X_{i}(t)\right\}\right) \\
& +\frac{\Delta t^{4}}{24} \frac{d^{2}}{d t^{2}} G_{i}\left(\left\{X_{i}(t)\right\}\right)+\frac{\Delta t^{5}}{120} \frac{d^{3}}{d t^{3}} G_{i}\left(\left\{X_{i}(t)\right\}\right), \\
X_{i}(t-\Delta t)=X_{i}(t)- & -\Delta t \frac{d}{d t} X_{i}(t)+\frac{\Delta t^{2}}{2} G_{i}\left(\left\{X_{i}(t)\right\}\right)-\frac{\Delta t^{3}}{6} \frac{d}{d t} G_{i}\left(\left\{X_{i}(t)\right\}\right) \\
& +\frac{\Delta t^{4}}{24} \frac{d^{2}}{d t^{2}} G_{i}\left(\left\{X_{i}(t)\right\}\right)-\frac{\Delta t^{5}}{120} \frac{d^{3}}{d t^{3}} G_{i}\left(\left\{X_{i}(t)\right\}\right),
\end{aligned}
$$

donde $\Delta t^{n} \equiv(\Delta t)^{n}$. Si a continuación tomamos (D.2)+(D.3), el resultado es

$$
X_{i}(t+\Delta t)+X_{i}(t-\Delta t)=2 X_{i}(t)+\Delta t^{2} G_{i}\left(\left\{X_{i}(t)\right\}\right)+\frac{\Delta t^{4}}{12} \frac{d^{2}}{d t^{2}} G_{i}\left(\left\{X_{i}(t)\right\}\right) .
$$

En esta expresión y en las subsiguientes el último término del lado derecho es el error local de truncamiento de mayor orden, el cual se incluye para efectos de comparación. Ahora bien, si despreciamos los términos de orden $O\left(\Delta t^{4}\right)$ obtenemos 
la forma explicita del algoritmo de Verlet

$$
X_{i}(t+\Delta t)=2 X_{i}(t)-X_{i}(t-\Delta t)+\Delta t^{2} G_{i}\left(\left\{X_{i}(t)\right\}\right)
$$

el cual calcula correctamente las posiciones hasta tercer orden en $\Delta t$. Este algoritmo ha sido el más utilizado en estudios de dinámica molecular debido a un buen número de razones. La principal de ellas es que es algoritmicamente estable [121] para valores relativamente grandes de $\Delta t$. Esto quiere decir que la expresión (D.5) no amplifica los errores que se van cometiendo de un paso de integración al siguiente (las posiciones se calculan correctamente hasta $O\left(\Delta t^{3}\right.$ ) incluso para tiempos de cómputo muy grandes). Además, su simplicidad lo hace muy eficiente en términos de tiempo de CPU y de almacenamiento. Sin embargo, la expresión (D.5) sólo calcula la trayectoria en el espacio de configuración del sistema. Para calcular las velocidades $V_{s}(y$, por lo tanto, la trayectoria completa en el espacio fase) podemos tomar (D.2)-(D.3), con lo que obtenemos

$$
V_{i}(t)=\frac{1}{2 \Delta t}\left[X_{i}(t+\Delta t)-X_{i}(t-\Delta t)\right]-\frac{\Delta t^{2}}{6} \frac{d}{d t} G_{i}\left(\left\{X_{i}(t)\right\}\right),
$$

la cual permite calcular la velocidad en el instante $t$ utilizando la información disponible en el intervalo $2 \Delta t$. Otra forma equivalente de expresarlas es por medio de las velocidades que se obtienen a partir de la información disponible en el intervalo $\Delta t$ y que están definidas como

$$
X_{i}(t+\Delta t)-X_{i}(t) \equiv(\Delta t) V_{i}(t+\Delta t / 2)
$$

con lo que se obtiene

$$
V_{i}(t)=\frac{1}{2}\left[V_{i}(t+\Delta t / 2)+V_{i}(t-\Delta t / 2)\right]-\frac{\Delta t^{2}}{6} \frac{d}{d t} G_{i}\left(\left\{X_{i}(t)\right\}\right)
$$

El primer inconveniente que encontramos siguiendo esta metodología es que las velocidades son calculadas con una precisión inferior a aquella con la cual se calculan las posiciones. Esto plantea problemas muy serios si, como es nuestro caso, estamos interesados en propiedades que dependan precisamente de las velocidades, tales como la temperatura cinética y las funciones de autocorrelación del momento, entre otras. Como los errores locales de truncamiento dependen directamente del tamaño del intervalo $\Delta t$, éstos disminuyen si tomamos un valor de $\Delta t$ suficientemente pequeño (con el sistema de unidades elegido para plantear la forma 
del Hamiltoniano (5.4) siempre tendremos $\Delta t<1$ ). Pero en la práctica es importante utilizar el paso de integración más grande posible de tal manera que el muestreo del espacio fast: sea lo más eficiente posible. Este hecho impone una restricción muy importante en cuanto al tamaño de $\Delta t$ que puede utilizarse en un problema particular. Finalmente, una restricción todavía más estricta la impone el número de osciladores utilizados para obtener los resultados reportados en este trabajo $\sim O\left(10^{5}\right)$, mismo que vuelve impráctico utilizar valores de $\Delta t$ para los cuales (D.6) o (D.8) proporcionan una aproximación razonable al valor exacto de las velocidades $\left\{V_{n}(t)\right\}$.

Una forma de mejoras está situación conservando el algoritmo de Verlet consiste en desarrollar las velocidades mismas (ya que éstas son las variables que nos interesa calcular con la mayor precisión posible) en serie de Taylor para varios instantes de tiempo, tanto anteriores como posteriores a $t$, obteniendo así expresiones de mayor orden que (D.8). Planteamos entonces los siguientes desarrollos en serie para las velocidades:

$$
\begin{aligned}
V_{i}(t+\Delta t / 2)= & V_{i}(t)+\frac{\Delta t}{2} G_{i}\left(\left\{X_{i}(t)\right\}\right)+\frac{\Delta t^{2}}{8} \frac{d}{d t} G_{i}\left(\left\{X_{i}(t)\right\}\right) \\
& +\frac{\Delta t^{3}}{48} \frac{d^{2}}{d t^{2}} G_{i}\left(\left\{X_{i}(t)\right\}\right)+\frac{\Delta t^{4}}{384} \frac{d^{3}}{d t^{3}} G_{i}\left(\left\{X_{i}(t)\right\}\right) \\
V_{i}(t-\Delta t / 2)= & V_{i}(t)-\frac{\Delta t}{2} G_{i}\left(\left\{X_{i}(t)\right\}\right)+\frac{\Delta t^{2}}{8} \frac{d}{d t} G_{i}\left(\left\{X_{i}(t)\right\}\right) \\
& -\frac{\Delta t^{3}}{48} \frac{d^{2}}{d t^{2}} G_{i}\left(\left\{X_{i}(t)\right\}\right)+\frac{\Delta t^{4}}{384} \frac{d^{3}}{d t^{3}} G_{i}\left(\left\{X_{i}(t)\right\}\right) \\
V_{i}(t-3 \Delta t / 2)= & V_{i}(t)-\frac{3 \Delta t}{2} G_{i}\left(\left\{X_{i}(t)\right\}\right)+\frac{9 \Delta t^{2}}{8} \frac{d}{d t} G_{i}\left(\left\{X_{i}(t)\right\}\right) \\
& -\frac{27 \Delta t^{3}}{48} \frac{d^{2}}{d t^{2}} G_{i}\left(\left\{X_{i}(t)\right\}\right)+\frac{81 \Delta t^{4}}{384} \frac{d^{3}}{d t^{3}} G_{i}\left(\left\{X_{i}(t)\right\}\right)
\end{aligned}
$$

Si ahora realizarnos la suma (D.9) $+($ D.10) obtenemos

$$
V_{i}(t)=\frac{1}{2}\left[V_{i}(t+\Delta t / 2)+V_{i}(t-\Delta t / 2)\right]-\frac{\Delta t^{2}}{8} \frac{d}{d t} G_{i}\left(\left\{X_{i}(t)\right\}\right)
$$

que corresponde a la expresión de primer orden (D.8) para $V_{i}(t)$ obtenida por medio del desarrollo en serie de las posiciones, pero con un término de error local de magnitud diferente al que aparece en la expresión (D.8). Las implicaciones de esta diferencia las discutiremos más adelante. 
Tomando la combinación 3(D.9) +6(D.10)-9(D.11) obtenemu:

$$
V_{i}(t)=\frac{1}{8}\left[3 V_{i}(t+\Delta t / 2)+6 V_{i}(t-\Delta t / 2)-V_{i}(t-3 \Delta t / 2)\right]-\frac{\Delta t^{\prime}}{16} d l^{2} G_{i}\left(\left\{X_{i}(t)\right\}\right)
$$

Esta nueva expresión para el cálculo de las velocidades es correctin hasta segundo orden en $\Delta t$, por lo cual es inherentemente superior a (D.8) comm estimador de las velocidades.

Siguiendo este mismo esquema es posible derivar una expresi'm para $V_{i}(t)$ que sea correcta hasta tercer orden en $\Delta t$. De hecho, Amini y Fincham [136] han derivado 12 expresiones distintas para el cálculo de la temperatura cinética por medio de las velocidades y que son correctas hasta distintos órdens's al tomar los desarrollos en serie de las posiciones, velocidades y de la energía cinética misma, inclusive. Sin embargo, existe un problema con todos estos drxirrollos de más alto orden en $\Delta t$, ya que las expresiones resultantes son muy presas para valores pequeños del paso de integración, pero se vuelven numéricment inestables para valores grandes de $\Delta t$ [137]. Quedúndonos entonces con las exprannes de primer orden en $\Delta t$ (esto es, cuando el error local de truncamiento es orden $\Delta t^{2}$ ) se obtienen las mismas expresiones para las velocidades (Ecs. (D.8, , (D.12), como ya vimos), pero con diferentes coeficientes en los términos del err." "cal. A mayor orden estos dos enfoques nos llevan a diferentes expresiones pa: $V_{i}$ así como a diferentes errores locales de truncamiento. Estas diferencias s coeficientes surgen debido a que las dos metodologías corresponden a prot. . was de valores iniciales ligeramente distintos, en los cuales las coordenadas $y$. elocidades se especifican de manera exacta, pero distinta en ambos casos, $\%$ los primeros pasos de integración. Para resolver un auténtico problema de con cicones iniciales se deben de hacer suposiciones adicionales, como ilustraremos nis adelante. Por lo tanto, ninguno de estos dos enfoques es intrínsecamente supe $:$ al otro.

Debido a todos estos factores podemos decir que la mejor fo. a de elegir la manera de calcular las velocidades tiene que basarse en los resu fos obtenidos en un problema particular. Extensas simulaciones reportadas Amini y Fincham [136] muestran que, tanto para el problema de dos oscilads $*$, unidimensionales interaccionando con un potencial Coulombiano repulsivo cs: $:$ para el de un fluido molecular caracterizado por un potencial de Lennard-Jont $\quad 25$, los valores tanto de la energía total instantánea como de la temperatura ca cabtenidos con la expresión (D.8) están sistemáticamente subestimados, mir as que la expresión (D.13) sobreestima esas mismas cantidades. Puesto qut a desviaciones 
son comparables en magnitud pero tienen signos opuestos, estos autores proponen un procedimiento bastante obvio, aunque totalmente ad hoc, para construir un mejor estimador de la energía cinética que consiste en tomar el promedio de las expresiones para las velocidades (D.8) y (D.13), obteniéndose

$$
V_{i}(t)=\frac{1}{16}\left[7 V_{i}(t--\Delta t / 2)+10 V_{i}(t-\Delta t / 2)-V_{i}(t-3 \Delta t / 2)\right]
$$

Si a esta última expresión la escribimos en términos de las posiciones $X_{i}$ que van siendo calculadas por medio de (D.5), obtenemos finalmente una fórmula que nos permite calcular los momentos $\left\{P_{i}(t)\right\}$ del sistema como

$P_{i}(t)=\frac{M_{i}}{16 \Delta t}\left[7\left[X_{i}(t+\Delta t)-X_{i}(t)\right]+10\left[X_{i}(t)-X_{i}(t-\Delta t)\right]-X_{i}(t-\Delta t)+X_{i}(t-2 \Delta t)\right]$.

El esquema completo de integración consiste entonces en utilizar (D.5) para calcular las posiciones $X_{i}(t+\Delta t)$ a partir de la posición actual $X_{i}(t)$ y la posición previa $X_{i}(t-\Delta t)$, para a continuación calcular el momento $P_{i}(t)$ en el instante actual a partir de las posiciones en los dos instantes inmediatamente anteriores, $X_{i}(t-\Delta t)$ y $X_{i}(t-2 \Delta t)$ por medio de (D.15). Pero entonces notamos que las posiciones y momentos iniciales $\left\{X_{i}(0), P_{i}(0)\right\}$ no son suficientes para iniciar el cálculo; es necesario hacer suposiciones adicionales para obtener las posiciones en el instante inmediatamente anterior a $t=0 \mathrm{y}$ poder inicializar asi nuestro esquema de integración. La forma más directa de proceder ( $\mathrm{y}$ que es la que de hecho hemos utilizado), consiste en obtener las posiciones en el instante $-\Delta t$ a partir de un integrador de Euler de la forma $X_{i}(-\Delta t)=X_{i}(0)-\left(P_{i}(0) / M_{i}\right) \Delta t$, donde $\left\{X_{i}(0)=0\right\}$ y los momentos iniciales $\left\{P_{i}(0)\right\}$ satisfacen ciertas características ya explicadas en el capítulo 5. Para el primer paso de integración se utiliza (D.6) de tal manera que, a partir del segundo paso, ya se tengan las posiciones en los dos instantes previos necesarios para aplicar el algoritmo (D.15) en todos los instantes subsecuentes.

Las trayectorias en el espacio fase de nuestro modelo calculadas a partir del esquema definido por (D.5) y (D.15) están además sujetas al efecto de los errores de redondeo que surgen debido a la implementación particular que se haga del algoritmo. Algunas de las fuentes de este tipo de errores están relacionadas al número de cifras significativas mantenidas en cada etapa del cálculo, al orden en que estos cálculos sor efectuados en la práctica y a cualquier aproximación utilizada en la evaluación de las funciones involucradas (potencias, raíces, etc.), entre otras. Por mucho, la fuente más importante de los errores de redondeo es 
la cantidad de cifras significativas que una cierta computadora utiliza para representar números de punto flotante. Para estudiar el efecto de esta fuente de error en la solución de nuestro sistema particular hicimos dos implementaciones diferentes del algoritmo: una en la que se utilizó una representación de números de punto flotante en doble precisión con longitud de palabra de 32 bits y otra con 64 bits. Los resultados de ambas implementaciones muestran diferencias significativas cuando $\epsilon \gg 1$ (precisamente el rango de $\epsilon$ que nos interesa estudiar), por lo que todos los calculos reportados en este trabajo fueron realizados utilizando la aritmética de 64 bits. El efecto de otras posibies fuentes de error de redondeo, tales como el nivel de optimizacion utilizado para generar el cónigo ejecutable (y que es necesario debido al tamaño de nuestro sistema), parece ser despreciable según todos los resultados obtenidos en pruebas preliminares. Como una última comprobación de la validez de mestro esquema de integración, mencionemos que el valor de la temperatura cinética para el caso $c=10\left(T_{c i n} \approx 11.61\right)$ obtenido por medio de (D.5) y (D.15) coincide con el valor ya reportado en la literatura [115] y que fué obtenido utilizando un método de integración más elaborado que el nuestro [138]. 


\section{Bibliografía}

[1] D. Dürr y H. Spohn, "Brownian motion and microscopic chaos," Nature (London) 394, 831 (1998); P. Gaspard, M. E. Briggs, M. K. Francis, J. V. Sengers, R. W. Gammon, J. R. Dorfman y R. V. Calabrese, "Experimental evidence for microscopic chacs," ibid., 865 (1998)

[2] C. P. Dettmann, E. G. D. Cohen y H. van Beijeren, "Microscopic chaos from Brownian motion?," Nature (London) 401, 875 (1999) http://arXiv.org/abs/nlin.CD/9904041

[3] C. P. Dettmann y E. G. D. Cohen, "Microscopic chaos and diffusion," J. Stat. Phys. 101, 775 (2000) http://arXiv.org/abs/nlin/0001062

[4] C. P. Dettmann y E. G. D. Cohen, "Note on chaos and diffusion," Preprint, http://arXiv.org/abs/nlin/0011025

[5] J. W. Gibbs Elementary Principles in Statistical Mechanics, Ox Bow Press, Conneticut, 1981 (reimpresión de la edición de 1902)

[6] L. Boltzmann Lectures on Gas Theory, Dover, New York, 1995

[7] E. G. D. Cohen, "Boltzmann and statistical mechanics," Preprint, http://arXiv.org/abs/cond-mat/9608054

[8] E. Braun, "Movimiento Browniano," Ciencia 35, 97 (1984)

[9] S. G. Brush The Kird of Motion We Call Heat, North-Holland, Amsterdam, 1976

[10] L. Boltzmann, "Entgegnung auf die wärmetheoretischen betrachtungen des Hern. E. Zermelo," Ann. Phys. 57, 773 (1896); reimpreso y traducido en: S. G. Brush Kinetic Theory, Pergamon Press, Oxford (1966) 
[11] A. Einstein, "Üher die von der molekularkinetischen theorie der wäsme geforderte bewegung von in ruhenden flüssigkeiten suspendierten teilchen," Ann. Thys. 17, 349 (1905); reimpreso y traducido en: Investigations on the Theory of Brownian Motion, ed. R. Fürth, Dover, New York, 1956

[12] M. von Smoluchowski, "Zarys teoryi kinetycanej ruch browna i roztworów metnych," Rozprawy Kraków A46, 257 (1906)

[13] D. S. Lemons y A. Gythel, "Panl Langevin's paper "On the theory of Brownian motion" "Sur la theorie du mouvement brownien," C. R. Acad. Sci. (Paris) 146, 330 (1908)]," Am. J. Phys. 65, 1079 (1997)

[14] D. T. Gillespie, "Fluctuation and dissipation in Brownian motion," Am. J. Phys. 61, 1077 (1993); B. G. de Grooth, "A simple model for Brownian motion leading to the Langevin equation," Am. J. Phys. 67, 1248 (1999); P. Grassia, "Dissipation, fuctuations, and conservation laws," Am. J. Phys. $69,113(2001)$

[15] D. T. Gillespie, "The mathematics of Brownian motion and Johnson noise," Arn. J. Phys. 64, 225 (1996)

[16] J. M. Sancho, M. San Miguel, S. L. Katz y J. D. Gunton, "Analytical and numerical studies of multiplicative noise," Phys. Rev. A 26, 1589 (1982) Apéndice $\mathrm{A}$.

[17] D. T. Gillespie, "Exact numerical simulation of the Ornstein-Uhlenbeck process and its integral," Phys. Rev. E 54, 2084 (1996)

[18] S. Chandrasekhar, "Stochastic problems in physics and astronomy," Rev. Mod. Phys. 15, 1 (1943); reimpreso en: Selected Papers in Noise and Stochastic Processes, ed. N. Wax, Dover, New York, 1954

[19] J. G. Kirkwood, "The statistical mechanical theory of transport processes. I. General Theory," J. Chem. Phys. 14, 180 (1946)

[20] J. Lebowitz y E. Rubin, "Dynamical study of Brownian motion," Phys. Rev. 131, $2381(1963)$

[21] J. Lebowitz y P. Résibois, "Microscopic theory of Brownian motion in an oscillating field; Connection with macroscopic theory," Phys. Rev. A 139, $1101(196 j)$ 
[22] P. Résibois y R. Dęvis, "Transport equation of a Brownian particle in an external field," Physica (Amsterdam) 30, 1077 (1964)

[23] P. Mazur e I. Oppenheim, "Molecular theory of Brownian motion," Physica (Amsterdam) 50, 24:1 (1970)

[24] R. Zwanzig, "Elementary derivation of time-correlation formulas for transport coefficients," J. Chem. Pbys. 40, 2527 (1964)

[25] S. Nordholm y R. Zwanzig, "A systematic derivation of exact generalized Brownian motion theory," J. Stat. Phys. 13, 347 (1975)

[26] R. Zwanzig, "Ensarable method in the theory of irreversibility," J. Chem. Phys. 33, 1338 (1960)

[27] H. Mori, "Transport, collective motion, and Brownian motion," Prog. Theor. Phys. 33, 423 (1965)

[28] H. Mori, "A continued-fraction representation of the time-correlation functions," Prog. Theor. Phys. 34, 399 (1965)

[29] L. F. Rull, E. de Miguel, J. J. Morales y M. J. Nuevo, "Brownian motion in an isothermal-isobaric bath: Mass and size dependence," Phys. Rev. A 40, 5856 (1989)

[30] M. S. Green, "Markoff random processes and the statistical mechanics of time-dependent phenomena," J. Chem. Phys. 20, 1281 (1952); "ibid. II. Irreversible Processes in Fluids," ibid 22, 398 (1954)

[31] I. A. Michaels e I. Oppenheim, "Long-time tails and Brownian motion," Physica $A$ 81, $221(197 . j)$

[32] B. J. Alder y T. E. Wainwright, "Velocity autocorrelations for hard spheres," Phys. Rev. Lett. 18, 988 (1967)

[33] D. Levesque y W. T'. Ashurst, "Long-time behavior of the velocity autocorrelation function for a fluid of soft repulsive particles," Phys. Rev. Lett. 33, 277 (1974)

[34] R. F. Fox, "Long-time tails and diffusion," Phys. Rev. A 27, 3216 (1983) 
[35] J. R. Dorfman, T. R. Kirkpartick y J. V. Sengers, "Generic long-range correlations in molecular fluids," Ann. Rev. Phys. Chern. 45, 213 (1994)

[36] R. Vogelsang y C. Hoheisel, "Determination of the friction coefficient via the force autocorrelation function. A molecular dynamics investigation for a dense Lennard-Jones fluid," J. Stat. Pbys. 47, 193 (1987)

[37] L. F. Rull, J. J. Morales y M. J. Nuevo, "Friction coefficient of a Brownian particle: Dependence on size and mass," Phys. Lett. A 167, 65 (1992)

[38] R. 1. Cukier y J. M. Deutch: "Microscopic theory of Brownian motion: The multiple-time-scale point of view," Phys. Rev. 177, 240 (1969)

[39] L. Bocquet, J. Piasecki y J. P. Hansen, "On the Brownian motion of a massive sphere suspended in a hard-sphere fluid. I. Multiple time-scale analysis and microscopic expression for the friction coefficient," J. Stat. Phys. 76, 505 (1994)

[40] L. Bocquet, J.P. Hansen y J. Piasecki, "On the Brownian motion of a massive sphere suspended in a hard-sphere huid. II. Molecular dyramics estimates of the friction coefficient," J. Stat. Phys. 76, 527 (1994)

[41] A. Pérez-Madrid, I. M. Rubí y P. Mazur, "Brownian motion in the presence of a temperature gradient," Physica A 212, 231 (1994)

[42] P. Mazur y E. Montroll, "Poincaré cycles, ergodicity, and irreversibility in assemblies of coupled harmonic oscillators," J. Math. Phys. 1, 70 (1960)

[43] E. Teramoto, "Heat flow in the linear chain of harmonically coupled particles," Prog. Theo. Phys. 28, 1059 (1962); Z. Rieder, J. L. Lebowitz y E. Lieb, "Properties of a harmonic crystal in a stationary nonequilibrium state," $J$. Math. Phys. 81073 (1967)

[44] R. J. Rubin, "Statistical dynamics of simple cubic lattices. Model for the study of Brownian motion," J. Math. Phys. 1, 309 (1960)

[45] H. Nakazawa, "A statistical mechanical model of Brownian motion," Prog. Theor. Phys. Suppl. No. 36, 172 (1966)

[46] H. Simanjuntak y L. Gunther, "Non-strchastic Langevin equation and the arrow of time," Physica A 147, 487 (1988) 
[47] P. C. Hemmer Dynamic and Stochastic Types of Motion in the Linear Chain, Tesis Doctoral, Trondheim, Noruega, 1959

[48] S. Takeno y J. Hori, "Continuum approximation for the motion of a heavy particle in one- and three-dimensional lattices," Prog. Theo. Phys. Suppl. No. 23,177 (1962)

[49] P. Ullersma, "An exactly solvable model for Brownian motion III. Motion of a heavy mass in a linear chain," Physica (Amsterdam) 32, 74 (1966)

[50] G. W. Ford, M. Keic y P. Mazur, "Statistical mechanics of assemblies of coupled oscillators," J. Math. Phys. 6, 504 (1965)

[51] P. E. Phillipson, "Effects of long range interactions in harmonically coupled systems I. Equilibrium fluctuations and diffusion," J. Math. Phys. 15, 2127 (1974)

[52] P. Mazur y E. Braun, "On the statistical mechanical theory of Brownian motion," Physica (Amsterdam) 30, 1973 (1964)

[53] M. H. Lee, "Can the velocity autocorrelation function decay exponentially?," Phys. Rev. Lett. 51, 1227 (1983); "Solutions of the generalized Langevin equation by a method of recurrence relations," Phys. Rev. B 26, 2547 (1982)

[54] A. A. Maradudin, E. W. Montroll, G. H. Weiss e I. P. Ipatova Theory of Lattice Dynamics in the Harmonic Approximation, 2a ed., Academic Press, New York, 1971

[55] J. Ford, "The Fermi-Pasta-Ulam problem: Paradox turns discovery," Phys. Rep. 213, 271 (1992)

[56] G. Parisi, "Planck's legacy to statistical mechanics," Preprint http://arXiv.org/abs/cond-mat/0101293

[57] E. Fermi, J. Pasta, S. Ulam y M. Tsingou, "Studies of nonlinear problems I," Los Alarnos Preprint LA-1940 (1955); publicado en: Collected Papers of Enrico Fermi, ed. E. Segré, Vol. II, University of Chicago Press, Chicago, 1965, p. 978; también reimpreso en: Nonlinear Wave Motion, ed. A.C. Newell, Lecture Notes in Applied Mathematics, Vol. 15, AMS, Providence, RI, 1974; también en: The Miny Body Problem, ed. D. C. Mattis, World Scientific, Singapore, 1993 , p. $851^{\circ}$ 
[58] R. L. Bivins, N. Metropolis y J. R. Pasta, "Nonlinear coupled oscillators: Modal equation approach," J. Comput. Phys. 12, 65 (1973)

[59] G. Benettin, "Ordered and chaotic motions in dynamical systems with many degrees of freedom," en: Molecular Dynarnics Simulation of Statistic. I Mechanical Systems, Varenna XCVII Course, eds. G. Ciccotti y W. G. Hoover, North-Holland, Amsterdam, 1986

[60] G. Benettin, G. Lo Vecchio y A. Tanenbaum, "Stochastic transition in two-dimensional Lennard-Jones systems," Phys. Rev. A 22, 1709 (1980); G. Benettin, A. Tanenbaum, "Ordered and stochastic behavior in a twodimensional Lennard-Jones system," Phys. Rev. A 28, 3020 (1983)

[61] L. Casetti, R. Livi, A. Macchi y M. Pettini, "Relaxation times in an anharmonic crystal with diluted impurities," Europhys. Lett. 32, 549 (1995)

[62] D. A. McQuarrie, Statistical Mechanics, Harper \& Row, New York, 1976

[63] F. M. Izrailev y B. V. Chirikov, "Statistical properties of a nonlinear string," Sov. Phys. Dokl. 1130 (1966); B.V. Chirikov, F.M. Izrailev y V.A. Tayursky, "Numerical experiments on the statistical behavior of dynamical systems with a few degrees of freedom," Comput. Phys. Comm. 5, 11 (1973)

[64] P. Bocchieri, A. Scotti, B. Bearzi y A. Loinger, "Anharmonic chain with Lennard-Jones interaction," Phys. Rev. 2, 2013 (1970)

[65] R. Livi, M. Pettini, S. Ruffo, M. Sparpaglione y A. Vulpiani, "Equipartition threshold in nonlinear large Hamiltonian systems: The Fermi-Pasta-Ulam Model," Phys. Rev. A 31, 1039 (1985)

[66] R. Livi, M. Pettini, S. Ruffo y A. Vulpiani, "Further results on the equipartition threshold in large nonlinear Hamiltonian systems," Phys. Rev. A 31, $2740(1985)$

[67] M. Pettinì y M. Landolf, "Relaxation properties and ergodicity breaking in nonlinear Hamiltonian dynamics," Phys. Rev. A 41, 768 (1990)

[68] I Kantz, R. Livi y S. Ruffo, "Equipartition thresholds in chains of anharII aic oscillators," J. Stat. Phys. 76, 627 (1994) 
[69] J. De Luca, A. J. Lichtenberg y M. A. Lieberman, "Time scale to ergodicity in the Fermi-Pasta-Clam system," Chaos 5, 283 (1995)

[70] J. De Luca, A. J. Licitenberg y S. Ruffo, "Energy transitions and time scales to equipartition in the Fermi-Pasta-Ulam oscillator chain," Phys. Rev. E 1, $2877(1995)$

[71] G. Parisi, "On the approach to equilibrium of a Hamiltonian chain of anharmonic oscillators," Europhys. Lett. 40, 357 (1997)

[72] L. Casetti, M. Cerruti-Sola, M. Pettini y E. G. D. Cohen, "The Fermi-PastaUlam problem revisited: Stochasticity thresholds in nonlinear Hamiltonian systems," Phys. Rev. E 55, 6566 (1997)

[73] V. I. Arnold y A. Avez Ergodic Problems of Classical Mechanics, Benjamin, New York, 1968

[74] Ya. G. Sinai, "Dynaraical systems with elastic reflections. Ergodic properties of dispersing billiards," Russ. Math. Surv. 25, 137 (1970)

[75] L. D. Landau y E. M. Lifshitz Statistical Physics, Pergamon Press, Oxford, 1958

[76] A. I. Khinchin Mathematical Foundations of Statistical Mechanics, Dover, New York, 1949

[77] H. Poincaré Les Méthodes Nouvelles de la Méchanique Celeste, Blanchard, París, 1897; E. Fermi, "Dimostrazione che in generale un sistema meccanico normale è quasi ergodico," Nuovo Cimento 25, 267 (1923); "Generalizzazione del teorema di Poincaré sopra la non esistenza di integrali uniformi di un sistema di equazioni canoniche normali," ibid 26, 105 (1923)

[78] A. N. Kolmogorov, "Preservation of conditionally periodic movements with small change in the Hamiltonian function," Dokl. Akad. Nauk, SSSR 98, 527 (1954); traducido al inglés y reimpreso en: Chaos, ed. Hao Bai-Lin, World Scientific, Singapore, 1984

[79] V. I. Arnold, "Proof of a theorem of A. N. Kolmogorov on the invariance of quasi-periodic motions under small perturbations of the Hamiltonian," Russ. Math. Surv. 18, 9 (1963) 
[80] J. K. Moser, "On invariant curves of area-preserving mappings on an annulus," Nachr. Akad. Wiss. Göttingen Math. Physik K1, 1 (1962)

[81] N. N. Nekhoroshev, "Behavior of Hamiltonian systems close to integrable," Funct. Anal. Appl. 5, 338 (1971); "An exponential estimate of the tme of stability of nearly-integrable Hamiltonian systems," Russ. Math. Surv. 32, I (1977)

[82] L. Chierchia y G. Gallavotti, "Srnooth prime integrals for quasi-integrable Hamiltonian systems," Nuovo Cimento B 67, 277 (1982); G. Benettin, L. Galgani, A. Giorgilli y J. M. Strelcyn, "A proof of Kolmoborov's theorem on invariant tori using canonical transformations defined by the Lie method," ibid. 79, 201 (1984)

[83] E. Wayne, "The KAM theory of systems with short range interactions, I," Commun. Math. Phys. 96, 311 (1984); "ibid., II," ibid. 96, 331 (1984)

[84] G. Benettin y G. Gallavotti, "Stability of motions near resonances in quasiintegrable Hamiltonian systems" J. Stat. Phys. 44, 293 (1986)

[85] G. Benettin, L. Galgani y A. Giorgilli, "A proof of Nekhoroshev's theorem for the stability times in nearly integrable Hamiltonian systerns," Cel. Mech. 37, 1 (1985)

[86] P. Lochak, "Effective speed of Arnold's diffusion and small denominators," Phys. Lett. A 143, 39 (1990)

[87] M. Hénon y C. Heiles, "The applicability of the third integral of motion; Some numerical experiments," Astron. J. 69, 73 (1964)

[88] G. H. Lunsford y J. Ford, "On the stability of periodic orbits for nonlinear oscillator systems in regions exhibiting stochastic behavior," J. Math. Phys. 13, $700(1972)$

[89] C. P. Enz, M. O. Hongler y C. V.Quach Thi, "Stability of linear chains with third-order anharmonicity," Helv. Phys. Acta 48, 787 (1975)

[90] G. P. Berman y A. R. Kolovskij, "The limit of stochasticity for a onedimensional chain of interacting oscillators," Sov. Phys.-JETP 60, 1116 (1984) 
[91] S. Isola, R. Livi y S. Ruffo, "Qualitative changes in Hamiltonian dynamics," Phys. Lett. A 112, 4.48 (1985)

[92] S. Isola, R. Livi y S. Ruffo, "Broken ergodicity and single-particle statistical properties," Europhys. Lett. 3, 407 (1987)

[93] M. Casartelli y S. Sello, "Low stochasticity and relaxation in the Hénon-Heiles model," Phys. Lett. A 112, 249 (1985)

[94] D. Thirumalai y R. L. Mountain, "Probes of equipartition in nonlinear Hamiltonian systems," J. Stat. Phys. 57, 789 (1989)

[95] G. Benettin, L. Galgani y J. M. Strelcyn, "Kolmogorov entropy and numerical experiments," Phys. Rev. A 14, 2338 (1976)

[96] R. Livi, A. Politi y S. Ruffo, "Distribution of characteristic exponents in the thermodynamic limi:," J. Phys. A 19, 2033 (1986)

[97] J. P. Eckmann y E. Wayne, "Lyapunov spectra for infinite chains of nonlinear oscillators," J. Stat. Phys. 50, 853 (1988)

[98] P. Poggi y S. Ruffo, "Exact solutions in the FPU oscillator chain," Physica D 103, 251 (1997)

[99] M. Pettini, "Geometrical hints for a nonperturbative approach to Hamiltonian dynamics," Phys. Rev E 47, 828 (1993); L. Casetti y M. Pettini, "Analytic computation of the strong stochasticity threshold in Hamiltonian dynamics using Riemannian geometry," Phys. Rev. E 48, 4320 (1993)

[100] L. Casetti, R. Livi y M. Pettini, "Gaussian model for chaotic instability of Hamiltonian flows," Phys. Rev. Lett. 74, 375 (1995)

[101] R. Livi, M. Pettini, S. Ruffo y A. Vulpiani, "Chaotic behavior in nonlinear Hamiltonian systems and equilibrium statistical mechanics," J. Stat. Phys. 48, 539 (1987)

[102] S. Lepri, R. Livi y A. Politi, "Heat conduction in chains of nonlinear oscillators," Phys. Rev. Lett. 78, 1896 (1997)

[103] S. Lepri, "Memory effects and heat transport in one-dimensional insulators," Preprint, http://aræiv.org/abs/cond-mat/0010188 
[104] D. T. Morelli, J. Heremans, M. Sakamoto y C. Uher, "Anisotropic heat conduction in diacetylenes," Phys. Rev. Lett. 57, 869 (1986); A. Smontara, I. C. Lasjaunias y R. Maynard, "Phonon Poiseuille flow in quasi-onedimensional single crystals," Phys. Rev. Lett. 77, 5397 (1996)

[105] H. Forsman y P. Anderson, "Thermal conductivity at high pressure of solid odd-numbered $n$-alkanes ranging from $\mathrm{C}_{9} \mathrm{H}_{20}$ to $\mathrm{C}_{19} \mathrm{H}_{40}, " J$. Chem. Phys. 80, 2804 (1984)

[106] T. S. Tighe, J. M. Worlock y M. L. Roukes, "Direct thermal conductance measurements on suspended monocrystalline nanostructures," Phys. Rev. Lett. 70, 2687 (1997)

[107] L. G. C. Rego y G. Kirczenow, "Quantized thermal conductance of dielectric quantum wires," Phys. Rev. Lett. 81, 232 (1998)

[108] D. M. Leitner y P. G. Wolynes, "Heat flow through an insulating nanocrystal," Phys. Rev. E 61, 2902 (2000)

[109] L. Galgani, A. Giorgilli, A. Martinoli y S. Vanzini, "On the problem of energy equipartition for large systems of the Fermi-Pasta-Ulam type: Analytical and numerical estimates," Physica D 59, 334 (1992); G. Huang y B. Hu, "Asymmetric gap soliton modes in diatomic lattices with cubic and quartic nonlinearity," Preprint, http://arXiv.org/abs/cond-mat/9712008

[110] J. De Luca, A. J. Lichtenberg y S. Ruffo, "Universal evolution to equipartition in oscillator chains," Phys. Rev E 54, 2329 (1996)

[111] R. Bourbonnais y R. Maynard, "Energy transport in one-and twodimensional anharmonic lattices with isotopic disorder," Phys. Rev. Lett. 64, 1397 (1990)

[112] L. Verlet, "Computer "experiments" on classical fluids. I. Thermodynamical properties of Lennard-Jones molecules," Phys. Rev. 159, 98 (1967)

[113] C. Albasio, M. Casartelli y P. Marenzoni, "Thermodynamic limit beyoid the stochasticity threshold in nonlinear chains," Phys. Lett. A 183, 305 (1993)

[114] C. Albasio, M. Casartelli y P. Marenzoni, "Nearly separable behavior of Fermi-Pasta-Ulam chains through the stochasticity threshold," J. Stat. Phys. 79, $451(1995)$ 
[115] C. Giardinà y R. Livi, "Ergodic properties of microcanonical observables," J. Stat. Phys. 91, 1027 (1998) bttp://arXiv.org/abs/chaordyn/9709015

[116] B. I. Henry y J. Gindlay, "Reversible approach to statistical equilibrium in a nonlinear chain: An ensamble study," Physica D 28, 49 (1987)

[117] A. Suárez, R. Silbey e I. Oppenheim, "Memory effects in the relaxation of quantum open systems," J. Chem. Phys. 97, 5101 (1992)

[118] V. Romero-Rochín y E. González-Tovar, "Commerts on some aspects of Boltzmann $H$ theorem using reversible molecular dynamics," J. Stat. Phys. 89, 735 (1997)

[119] K. Lindenberg y V. Seshadri, "Dissipative contributio"s of internal multiplicative noise," Physica A 115, 483 (1981)

[120] J. Mencia Bravo, R. M. Velasco y J. M. Sancho, "A classical particle in heat bath under the influence of external noise," J. Math. Phys. 30, 2023 (1989)

[121] J. M. Haile Molecular Dynamics Simulation: Elementary Methods, John Wiley \& Sons, New York, 1992

[122] E. Omerti, M. Ronchetti y D. Dürr, "Numerical evidence for mass dependence in the diffusive behavior of the "heavy particle" on the line," J. Stat. Phys. 44, 339 (1986i)

[123] R. Zwanzig y N. A.lawadi, "Statistical error due to finite time averaging in computer experiments," Phys. Rev. 182, 280 (1969)

[124] K. Yoshimura, "St:ong stochasticity threshold in some anharmonic lattices," Physica D 104, 148 (1997)

[125] A. Gavrielides, T. Kottos, V. Kovanis y G. P. Tsironis, "Spatiotemporal organization of coupled nonlinear pendula through impurities," Phys. Rev. E 58, 5529 (1998)

[126] M. Weiss, T. Kottos y T. Geisel, "Taming chaos by impurities in twodimensional oscillator arrays," Phys. Rev. E 63, 056211 (2001)

[127] T. Morita y H. Mori, "Kinetic and hydrodynamic scalings in an exactlysoluble model for the Brownian motion," Prog. Theo. Phys. 56, 498 (1976) 
[128] E. Braun, "A model of Brownian dynamics for colloidal suspensions," Physica A 103, 325 (1980)

[129] L. Casetti y A. Macchi, "Geometric dynamical observables in rare gas crystals," Phys. Rev. E 55, 2539 (1997)

[130] S. Kashiwamura y E. Teramoto, "Effect of an isotopic impurity on the heat flow in one-dimensional coupled harmonic oscillators," Prog. Theo. Phys. Supp. No. 23, 207 (1962)

[131] R. Reigada, A. Sarmiento y K. Lindenberg, "Energy flow in Fermi-PastaUlam arrays," Preprint, http://arXiv.org/abs/cond-mat/0109361

[132] G. Casati y J. Ford, "Stochastic transition in the unequal-mass Toda lattice," Phys. Rev. A 12, 1702 (1975)

[133] T. Dauxois, S. Ruffo, A. Torcini y T. Cretegny, "Localization and equipartition of energy in the $\beta$-FPU chain: Chaotic breathers," Physica $D$ 121, 106 (1998) http://arXiv .org/abs/cond-mat/9709204

[134] J. P. Bouchaud y A. Georges, "Anomalous diffusion in disordered media statistical mechanisms, models and physical applications," Phys. Rep. 195, 127 (1990); J. Klafter, M. F. Shlesinger y G. Zumofen, "Beyond Brownian motion," Physics Today 48, 33 (1996)

[135] W. Heitler The Quantum Theory of Radiation, 3a ed., Oxford University Press, Oxford, 1954

[136] M. Amini y D. Fincham, "Evaluation of temperature in molecular dynamics simulations," Comput. Phys. Commun. 56, 313 (1990)

[137] R. W. Hockney y J. W. Eastwood Computer Simulation Using Particles, McGraw-Hill, New York, 1981, Cap. 4

[138] L. Casetti, "Efficient symplectic algorithms for numerical simulations of Hamiltonian flows," Phys. Scr. 51, 29 (1995) 